\title{
Funções de Transferência com \\ Coeficientes Variando no Tempo
}

\author{
Maria Sílvia de Assis Moura \\ TESE APRESENTADA \\ AO INSTITUTO DE MATEMÁTICA E ESTATÍSTICA \\ DA UNIVERSIDADE DE SÃO PAULO \\ PARA OBTENÇÃO DO GRAU DE \\ DOUTOR EM ESTATÍSTICA
}

Área de Concentração: Estatística

Orientador: Prof. Dr. Pedro Alberto Morettin

Co-Orientadora: Profa. Dra. Clélia Maria de Castro Tolói 


\section{FUNÇÕES DE TRANSFERENNCIA COM COEFICIENTES \\ VARIANDO NO TEMPO.}

Este exemplar corresponde à redação final da tese devidamente corrigida e defendida por Maria Sílvia de Assis Moura e aprovada pela comissão julgadora.

São Paulo, 12 de abril de 2005.

Banca Examinadora:

- Prof. Dr. Pedro Alberto Morettin (Presidente) - IME - USP

- Profa. Dra. Clélia Maria de Castro Toloi - IME - USP

- Profa. Dra. Silvia Regina Costa Lopes - UFRGS

- Prof. Dr. Reinaldo Castro Souza - PUC - RJ

- Prof. Dr. Aluísio de Souza Pinheiro - IME - UNICAMP 


\section{Agradecimentos}

À Clélia Maria de Castro Toloi e ao Pedro Alberto Morettin por terem me dado a honra de serem meus orientadores.

Aos colegas e amigos do Departamento de Estatística da UFSCar e ao CNPq, pelo apoio finaceiro, por possibilitarem a realização desse trabalho.

À Maria Aparecida de Paiva Franco e ao Antonio Ribeiro Franco pela cessão dos dados aqui utilizados.

Às meninas da República WAM-WAM e seus agregados pela amizade constante, apesar da distância.

Aos colegas do IME-USP: Carolina, Alice, Fábio, Maité, Said, Mário, Adriano, Domingo, Anderson e Marcelo pela conversas, discussões e esclarecimentos especialmente regados com um cafezinho de Dona Idalvina e Dona Jovina.

A Delhi, Susana e Maria pela amizade verdadeira, a Luciana e Suzi que, sem elas, esse trabalho nem teria começado.

Aos meus alunos pelo desafio constante.

Aos meus professores em especial Susana Foglio, Jorge A. Achcar, Anníbal P. Sant'Anna e Júlio M. Singer por todo o conhecimento transmitido que possibilita- 
ram a minha formação e constante aprimoramento.

À toda minha familia pelo suporte constante durante a toda a realização desse trabalho, em especial a Célia e Antônio de Pádua, meus pais.

À Ná, minha madrinha e avó, que infelizmente não compartilhou da alegria de ver o final desse trabalho.

Ao César, por tudo. 
"It's good to have an end to journey towards; but in the end, it is the journey itself that makes the difference." Ursula K. Le Guin iv 


\section{Resumo}

Neste trabalho apresentamos o modelo de função de transferência quando a série de entrada é localmente estacionária segundo a definição de processos localmente estacionários apresentada por Dahlhaus (1997).

Para estimar os coeficientes da função de transferência que neste caso, variam com o tempo, propusemos um estimador em dois estágios. Em cada estágio fizemos uma regressão da série de saída sobre as decomposições em ondaletas das série de entrada e defasagens da série de saída. Mostramos que os estimadores dos coeficientes, na regressão do primeiro estágio, têm distribuição normal.

Para suavizar as funções estimadas dos coeficientes da função de transferência usamos a técnica de limiarização dos coeficientes estimados por meio do limiar duro universal.

Apresentamos uma aplicação aos dados diários de temperatura máxima e precipitação atmosférica em Ribeirão Preto, SP, no período de 01 de janeiro de 1988 a 25 de dezembro de 1996 . 


\section{Abstract}

We consider the transfer function model with a locally stationary process as an input series following the definition of locally stationary process of Dahlhaus (1997).

In order to estimate the transfer function coefficients, which are time-varying, we propose a two-stage estimator. In each stage, we fit a regression of the output series on a linear combination of lagged input and output series. We prove that, in the first stage of this regression, the transfer function coefficient estimators have an asymptotic normal distribuition.

In order to re-construct the time-varying function of interest we used hard thresholds to smooth the estimated wavelet coefficients.

An application is done to daily data of maximum temperature and atmospheric precipitation in Ribeirão Preto, SP, between January 1st, 1988 and December 25th, 1996. 


\section{Sumário}

1 Preliminares 1

1.1 Processos Estacionários . . . . . . . . . . . . . . . . . . . . . . . . . 1

1.2 Função de Transferência no Caso Estacionário . . . . . . . . . . . . 3

1.2 .1 Estabilidade . . . . . . . . . . . . . . . . . 3

1.3 Modelo a ser Tratado . . . . . . . . . . . . . . . . . 4

2 Noções Básicas $\quad 7$

2.1 Processos Localmente Estacionários . . . . . . . . . . . . . . 7

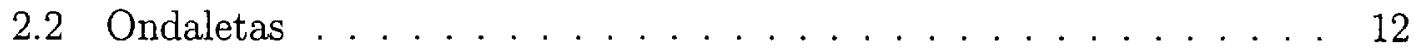

2.2 .1 Transformada de Ondaletas . . . . . . . . . 16

3 Estimação do Modelo de Função de Transferência 20

3.1 Estimadores Propostos . . . . . . . . . . . . . . . 20

3.2 Estimadores do Primeiro Estágio . . . . . . . . . . . . . . 23

3.2.1 Propriedades Estatísticas . . . . . . . . . . . . . . 29

3.3 Estimadores do Segundo Estágio. . . . . . . . . . . . . . . . . 43 
3.3.1 Propriedades Estatísticas . . . . . . . . . . . . 45

3.4 Intervalos . . . . . . . . . . . . . . . . . . 48

3.5 Generalizações . . . . . . . . . . . . . . . . . . . . 49

3.5.1 Estimadores do Primeiro Estágio . . . . . . . . . . . . 49

3.5.2 Estimadores do Segundo Estágio . . . . . . . . . . . . 52

4 Simulações e Exemplos $\quad 54$

4.1 Simulações . . . . . . . . . . . . . . . . . . . . . 54

4.2 Caso $1 \ldots \ldots \ldots \ldots \ldots \ldots$

4.3 Caso $2 \ldots \ldots \ldots \ldots \ldots$. . . . . . . . . . . . . . . 60

4.4 Exemplo Real . . . . . . . . . . . . . . . . . . . 65

5 Conclusōes e Trabalhos Futuros $\quad 82$

A Normas e Lemas $\quad 84$

A.1 Resultados de Normas de Matrizes . . . . . . . . . . . . . . . 84

A.1.1 Definições . . . . . . . . . . . . . . . . 84

A.1.2 Equivalência entre Normas . . . . . . . . . . . . 85

A.2 Normalidade de Formas Quadráticas . . . . . . . . . . . . 85

A.3 Lemas . . . . . . . . . . . . . . . . . . 87

$\begin{array}{ll}\text { B Histogramas da Simulação } & 102\end{array}$

$\begin{array}{lr}\text { C Resultados } & 124\end{array}$ 
D Programa

Referências Bibliográficas 



\section{Capítulo 1}

\section{Preliminares}

\subsection{Processos Estacionários}

Segundo Box et al. (1994), um fenômeno estatístico envolvendo o tempo de acordo com leis probabilísticas é chamado processo estocástico. Uma particular realização desse processo é uma série temporal. Uma classe muito especial de processos estocásticos é a classe dos processos estocásticos estacionários. A palavra estacionário se refere a um estado particular do equilibrio estatístico. Um processo é dito ser estritamente estacionário se suas distribuições finito-dimensionais são invariantes no tempo.

Consideremos um processo estocástico $\left\{X_{t}, t \in \mathbb{Z}\right\}$, com média zero e variância finita, i.e.,

$$
\begin{aligned}
\mathbb{E}\left[X_{t}\right] & =0 \\
\boldsymbol{E}\left[\left|X_{t}\right|^{2}\right] & <\infty
\end{aligned}
$$


A função de autocovariância desse processo é definida como:

$$
R(s, t)=\boldsymbol{E}\left[X_{s} \cdot X_{t}^{*}\right]
$$

em que $X_{t}^{*}$ indica o complexo conjugado de $X_{t}$. Se $\left\{X_{t}, t \in \mathbb{Z}\right\}$ for fracamente estacionário, $R(s, t)$ será função apenas de $|s-t|$ e, pelo teorema de WienerKhintchine, $R(s, t)$ admite a representação

$$
R(s, t)=\int_{-\infty}^{\infty} e^{i \omega(t-s)} d H(\omega)
$$

sendo $H(\omega)$ o espectro integrado. Por outro lado, o processo $\left\{X_{t}, t \in \mathbb{Z}\right\}$ admite a representação espectral

$$
X_{t}=\int_{-\infty}^{\infty} e^{i \omega t} d Z(\omega)
$$

em que $Z(\omega)$ é um processo com incrementos ortogonais e $\boldsymbol{E}\left[|d Z(\omega)|^{2}\right]=d H(\omega)$.

Se $X_{t}$ não é estacionário, ele não poderá ser representado por (1.2) e sua função de autocovariância não será representada por (1.1).

Segundo Priestley (1981), para uma classe de processos estocásticos existe uma representação para $R(s, t)$ similar a $(1.1)$, bastando substituir $\left\{e^{i \omega t}\right\}$ por uma família mais genérica de funções, $\left\{\phi_{t}(\omega)\right\}$. A família $\mathcal{F}$ de funções $\left\{\phi_{t}(\omega)\right\}$ é definida em $\mathbb{R}$, indexada por $t$ e medida $\vartheta(\omega)$, tal que para cada $t, s$ a autocovariância $R(t, s)$ admite a representação

$$
R(s, t)=\int_{-\infty}^{\infty} \phi_{s}^{*}(\omega) \phi_{t}(\omega) d \mu(\omega)
$$

A representação da forma (1.3) será sempre possível no caso de o espaço paramétrico ser finito. Além disso, $\left\{X_{t}, t \in \mathbb{Z}\right\}$ admite a representação

$$
X_{t}=\int_{-\infty}^{\infty} \phi_{t}(\omega) d Z(\omega)
$$

em que $Z(\omega)$ indica um processo de incrementos ortogonais com $\mathbb{E}\left[|d(Z(\omega))|^{2}\right]=$ $d \vartheta(\omega)$. 
A teoria para analisar processos estacionários está muito bem estabelecida, enquanto que para processos não estacionários ainda há muito o que fazer.

\subsection{Função de Transferência no Caso Estacionário}

Consideremos $\left\{X_{t}, t=1,2, \ldots, T\right\}$ e $\left\{Y_{t}, t=1,2,3, \ldots, T\right\}$, séries temporais estacionárias de modo que $X_{t}$ seja a entrada e $Y_{t}$ a saída de um sistema dinâmico. Podemos ter qualquer relação ligando $Y_{t}$ a $X_{t}$, porém, estaremos interessados no caso de relação linear, isto é:

$$
\begin{aligned}
Y_{t} & =v_{0} X_{t}+v_{1} X_{t-1}+v_{2} X_{t-2}+\ldots \\
& =\left(v_{0}+v_{1} B+v_{2} B^{2}+\ldots\right) X_{t}=\mathbf{v}(B) X_{t}
\end{aligned}
$$

Dizemos que $\mathbf{v}(B)$ é a função de transferência do filtro e os coeficientes $v_{0}, v_{1}, v_{2}, \ldots$ são chamados função resposta de impulso do sistema, sendo que $B$ é o operador de defasagem. Podemos interpretar $v_{j}$ como a resposta no tempo $j \leq 0$ para um pulso unitário no tempo 0.

Esse modelo é tratado em vários textos, como por exemplo, Box et al. (1994).

\subsubsection{Estabilidade}

Se a série infinita $v_{0}+v_{1} B+v_{2} B^{2}+\ldots$ convergir para $|B| \leq 1$, ou de forma equivalente, se $v_{j}$ é absolutamente somável,

$$
\sum_{j=0}^{\infty}\left|v_{j}\right|<\infty
$$

então o sistema é dito estável. 
A condição (1.4) implica que uma alteração finita na entrada provoca uma alteração finita na saída.

Aqui há a necessidade de estimarmos muitos coeficientes $v_{j}$. Uma alternativa para encontrar um modelo parcimonioso é substituir $\mathbf{v}(B)$ por uma função racional da forma

$$
\mathbf{v}(B)=\frac{\boldsymbol{\omega}(B) B^{b}}{\boldsymbol{\delta}(B)}
$$

em que

$$
\boldsymbol{\omega}(B)=\omega_{0}+\omega_{1} B+\omega_{2} B^{2}+\ldots+\omega_{s} B^{s}
$$

e

$$
\delta(B)=1-\delta_{1} B-\delta_{2} B^{2}-\ldots-\delta_{r} B^{r}
$$

são polinômios de graus $s$ e $r$, respectivamente. $O$ valor de $b$ diferente de zero indica um atraso na resposta com relação à entrada, por isso $b$ é chamado parâmetro de defasagem. Com essa forma racional, o modelo será estável se as raízes da equação característica $\delta(B)=0$ caírem fora do círculo unitário.

Uma dificuldade no ajuste desse modelo é a identificação das ordens dos polinômios $\omega(B)$ e $\delta(B)$, bem como o valor de b. Box et al. (1994) apresentam uma alternativa através da função de correlação cruzada entre a série de entrada branqueada, isto é, a série de entrada é multiplicada por polinômios que a torne ruído branco e a série de saída também multiplicada pelos mesmos polinômios.

\subsection{Modelo a ser Tratado}

O modelo que trataremos neste trabalho é o da função de transferência com entrada $X_{t}$ e saída $Y_{t}$ não necessariamente estacionários; aqui os coeficientes de 
$\mathrm{v}_{t}(B)$ dependerão do tempo, isto é,

$$
\begin{aligned}
Y_{t} & =v_{0}(t) X_{t}+v_{1}(t) X_{t-1}+v_{2}(t) X_{t-2}+\ldots \\
& =\left(v_{0}(t)+v_{1}(t) B+v_{2}(t) B^{2}+\ldots\right) X_{t}=v_{t}(B) X_{t}
\end{aligned}
$$

Para o sistema ser estável, a série $v_{j}(t)$ deve ser absolutamente somável para todo $t$ :

$$
\sup _{1 \leq t \leq T} \sum_{j=0}^{\infty}\left|v_{j}(t)\right|<\infty .
$$

Podemos reescrever a função de transferência na forma

$$
\mathbf{v}_{t}(B)=\frac{\boldsymbol{\omega}_{t}(B) B^{b}}{\boldsymbol{\delta}_{t}(B)}
$$

com

$$
\omega_{t}(B)=\omega_{0}(t)+\omega_{1}(t) B+\omega_{2}(t) B^{2}+\ldots+\omega_{s}(t) B^{s}
$$

e

$$
\delta_{t}(B)=1-\delta_{1}(t) B-\delta_{2}(t) B^{2}-\ldots-\delta_{r}(t) B^{r}
$$

O modelo a ser estudado será,

$$
\begin{aligned}
Y_{t} & =v_{0}(t) X_{t}+v_{1}(t) X_{t-1}+v_{2}(t) X_{t-2}+\ldots \\
& =\left(v_{0}(t)+v_{1}(t) B+v_{2}(t) B^{2}+\ldots\right) X_{t} \\
& =v_{t}(B) X_{t} \\
& =\frac{\omega_{t}(B) B^{b}}{\delta_{t}(B)} X_{t}
\end{aligned}
$$

Aqui, $\omega_{j}(t), \quad j=0,1,2, \ldots, s$, são funções contínuas e limitadas, com derivadas contínuas e limitadas, satisfazendo as condições $\omega_{j}(u)=\omega_{j}(0), u<0$, e $\omega_{j}(u)=\omega_{j}(1), u>1, u=t / T$. Similarmente, $\delta_{j}(t), \quad j=0,1,2, \ldots, r$, são funções 
contínuas e limitadas, com derivadas contínuas e limitadas, com $\delta_{j}(u)=\delta_{j}(0), u<$ 0 , e $\delta_{j}(u)=\delta_{j}(1), u>1, u=t / T$. Note que estamos fazendo uma contração do tempo, de modo que $0<u \leq 1$. No capítulo 2 apresentamos a definição de Processos Localmente Estacionários de Dahlhaus e verificamos que, em uma função de transferência com a série de entrada localmente estacionária, a série de saída também será localmente estacionária. No capítulo 3 apresentamos estimadores para as funções que compõem a função de transferência, bem como suas propriedades estatísticas. Simulações são apresentadas no capítulo 4, bem como aplicações. No capítulo 5 discutiremos os resultados obtidos e estudos futuros. No apêndice constam resultados de álgebra linear e alguns lemas apresentados em Dahlhaus et al. (1999) adaptados para esse caso. 


\section{Capítulo 2}

\section{Noções Básicas}

\subsection{Processos Localmente Estacionários}

Dahlhaus (1997) apresenta a seguinte definição de não-estacionariedade.

Uma seqüência de processos estocásticos $X_{t, T}(t=1,2,3, \ldots, T)$ é chamada localmente estacionária com função de transferência $A$ e tendência $\mu$, se existe uma representação

$$
X_{t, T}=\mu\left(\frac{t}{T}\right)+\int_{-\pi}^{\pi} e^{i \lambda t} A\left(\frac{t}{T}, \lambda\right) d \xi(\lambda)
$$

com:

(i) $\xi(\lambda)$ é um processo estocástico com incrementos ortogonais em $[-\pi, \pi]$ com $\overline{\xi(\lambda)}=\xi(-\lambda), \mathbb{E}(\xi(\lambda))=0 \mathrm{e}$ $\operatorname{cum}\left\{d \xi\left(\lambda_{1}\right), d \xi\left(\lambda_{2}\right) \ldots d \xi\left(\lambda_{k}\right)\right\}=\eta\left(\sum_{j=1}^{k} \lambda_{k}\right) g_{k}\left(\lambda_{1} \ldots \lambda_{k-1}\right) d \lambda_{1} \ldots d \lambda_{k}$

onde $\operatorname{cum}\{\ldots\}$ denota o cumulante de ordem $k, g_{1}(\lambda)=0, g_{2}(\lambda)=1$, 
$\left|g_{k}\left(\lambda_{1}, \ldots, \lambda_{k-1}\right)\right| \leq$ const $_{k}$ para todo $k$ e $\eta(\lambda)=\sum_{j=-\infty}^{\infty} \delta(\lambda+2 \pi j)$ é uma extensão de período $2 \pi$ da função delta de Dirac.

(ii) Existe uma função $2 \pi$ periódica $A:[0,1] \times \mathbb{R} \rightarrow C \operatorname{com} A(u,-\lambda)=\overline{A(u, \lambda)}$. $A(u, \lambda)$ e $\mu(u)$ são supostas contínuas em $u$, sendo $u=t / T, t=1,2,3, \ldots, T$.

Mallat et al. (1998) apresentam uma outra definição para processos localmente estacionários:

Suponha que exista uma função $l(t)$ positiva tal que para todo $k \geq 0$ e $j \geq 0$ podemos encontrar $D_{k, j}$ satisfazendo

$$
\left|\partial_{t}^{k} \partial_{\omega}^{j} \mathbb{A}(t, \omega)\right| \leq D_{k, j} l^{j-k}(t)
$$

Se, para algum $\alpha<1 / 2$ e uma constante $\mathbb{A}, \forall(t, u) \in \mathbb{R}^{2}$,

$$
|l(t)-l(u)| \leq \mathbb{A}|t-u|^{\alpha}
$$

então $X_{t}$ é um processo localmente estacionário com representação

$$
X(t)=\frac{1}{2 \pi} \int_{-\infty}^{\infty} \mathbb{A}(t, \omega) e^{-i \omega t} d \hat{Z}(\omega)
$$

em que $\hat{Z}(\omega)$ é um processo de incrementos ortogonais.

Neste trabalho adotaremos a definição de Dahlhaus dada pela expressão (2.1).

Proposição 2.1. Considere o modelo de função de transferência

$$
Y_{t, T}=\mathbf{v}_{t}(B) X_{t, T}
$$

com (1.5) satisfeita e $\mathrm{v}_{t}(B)$ dada por (1.6). Se $X_{t, T}$ é localmente estacionário, então $Y_{t, T}$ também é localmente estacionário.

Prova. Considerando o sistema dinâmico estável temos que, para cada $t$ o polinômio $\delta_{t}(B)=1-\delta_{1}\left(\frac{t}{T}\right) B-\delta_{2}\left(\frac{t}{T}\right) B^{2}-\ldots-\delta_{r}\left(\frac{t}{T}\right) B^{r}$ pode ser fatorado na forma 


$$
\begin{aligned}
& \delta_{t}(B)= {\left[1-\bar{\delta}_{1}\left(\frac{t}{T}\right) B\right]\left[1-\bar{\delta}_{2}\left(\frac{t}{T}\right) B\right] \ldots\left[1-\bar{\delta}_{r}\left(\frac{t}{T}\right) B\right] \mathrm{e}, \text { então } } \\
& \delta_{t}^{-1}(B)=\left[1-\bar{\delta}_{1}\left(\frac{t}{T}\right) B\right]^{-1}\left[1-\bar{\delta}_{2}\left(\frac{t}{T}\right) B\right]^{-1} \ldots\left[1-\bar{\delta}_{r}\left(\frac{t}{T}\right) B\right]^{-1} \\
&=\left[\sum_{j_{1}=0}^{\infty}\left(\bar{\delta}_{1}\left(\frac{t}{T}\right) B\right)^{j_{1}}\right]\left[\sum_{j_{2}=0}^{\infty}\left(\bar{\delta}_{2}\left(\frac{t}{T}\right) B\right)^{j_{2}}\right] \ldots\left[\sum_{j_{r}=0}^{\infty}\left(\bar{\delta}_{r}\left(\frac{t}{T}\right) B\right)^{j_{r}}\right] \\
&=\sum_{j_{1}=0}^{\infty} \sum_{j_{2}=0}^{\infty} \ldots \sum_{j_{r}=0}^{\infty} \bar{\delta}_{1}^{j_{1}}\left(\frac{t}{T}\right) \bar{\delta}_{2}^{j_{2}}\left(\frac{t}{T}\right) \ldots \bar{\delta}_{r}^{j_{r}}\left(\frac{t}{T}\right) B^{j_{1}+j_{2}+\ldots+j_{r}} \\
&=\sum_{l_{r}=0}^{\infty} \sum_{l_{r-1}=0}^{l_{r}} \sum_{l_{r-2}=0}^{l_{r-1}} \ldots \sum_{l_{1}=0}^{l_{2}} \bar{\delta}_{1}^{l_{1}}\left(\frac{t}{T}\right) \bar{\delta}_{2}^{l_{2}-l_{1}}\left(\frac{t}{T}\right) \ldots \bar{\delta}_{r}^{l_{r}-l_{r-1}}\left(\frac{t}{T}\right) B^{l_{r}} .
\end{aligned}
$$

Dessa forma, temos que

$$
\begin{aligned}
\mathbf{v}_{t}(B)= & \frac{\omega_{t}(t)}{\delta_{t}(B)} B^{b} \\
= & \sum_{l_{r}=0}^{\infty} \sum_{l_{r-1}=0}^{l_{r}} \sum_{l_{r-2}=0}^{l_{r-1}} \ldots \sum_{l_{1}=0}^{l_{2}} \bar{\delta}_{1}^{l_{1}}\left(\frac{t}{T}\right) \bar{\delta}_{2}^{l_{2}-l_{1}}\left(\frac{t}{T}\right) \ldots \bar{\delta}_{r}^{l_{r}-l_{r-1}}\left(\frac{t}{T}\right) B^{l_{r}} \\
& {\left[\omega_{0}\left(\frac{t}{T}\right)+\omega_{1}\left(\frac{t}{T}\right) B+\ldots+\omega_{s}\left(\frac{t}{T}\right) B^{s}\right] B^{b} } \\
= & \sum_{l_{r}=0}^{\infty} \sum_{l_{r-1}=0}^{l_{r}} \sum_{l_{r-2}=0}^{l_{r-1}} \ldots \sum_{l_{1}=0}^{l_{2}} \bar{\delta}_{1}^{l_{1}}\left(\frac{t}{T}\right) \bar{\delta}_{2}^{l_{2}-l_{1}}\left(\frac{t}{T}\right) \ldots \bar{\delta}_{r}^{l_{r}-l_{r-1}}\left(\frac{t}{T}\right) \\
& {\left[\omega_{0}\left(\frac{t}{T}\right) B^{l_{r}+b}+\omega_{1}\left(\frac{t}{T}\right) B^{l_{r}+1+b}+\ldots+\omega_{s}\left(\frac{t}{T}\right) B^{l_{r}+s+b}\right] . }
\end{aligned}
$$

Considerando $X_{t, T}$ dado por (2.1), segue que

$$
\begin{aligned}
\mathrm{v}_{t}(B) X_{t, T}= & \sum_{l_{r}=0}^{\infty} \sum_{l_{r-1}=0}^{l_{r}} \sum_{l_{r-2}=0}^{l_{r-1}} \cdots \sum_{l_{1}=0}^{l_{2}} \bar{\delta}_{1}^{l_{1}}\left(\frac{t}{T}\right) \bar{\delta}_{2}^{l_{2}-l_{1}}\left(\frac{t}{T}\right) \ldots \bar{\delta}_{T}^{l_{r}-l_{r-1}}\left(\frac{t}{T}\right) \\
= & {\left[\omega_{0}\left(\frac{t}{T}\right) X_{t-l_{r}-b, T}+\omega_{1}\left(\frac{t}{T}\right) X_{t-l_{r}-1-b, T}+\ldots+\right.} \\
& \left.+\omega_{s}\left(\frac{t}{T}\right) X_{t-l_{r}-s-b, T}\right],
\end{aligned}
$$

com

$$
X_{t, T}=\mu_{X}\left(\frac{t}{T}\right)+\int_{-\pi}^{\pi} e^{i \lambda t} A_{X}\left(\frac{t}{T}, \lambda\right) d \xi(\lambda)
$$




\section{Então}

$$
\begin{aligned}
\mathbf{v}_{t}(B) X_{t, T}= & \sum_{l_{r}=0}^{\infty} \sum_{l_{r-1}=0}^{l_{r}} \sum_{l_{r-2}=0}^{l_{r-1}} \ldots \sum_{l_{1}=0}^{l_{2}} \bar{\delta}_{1}^{l_{1}}\left(\frac{t}{T}\right) \bar{\delta}_{2}^{l_{2}-l_{1}}\left(\frac{t}{T}\right) \ldots \bar{\delta}_{r}^{l_{r}-l_{r-1}}\left(\frac{t}{T}\right) \\
& {\left[\omega_{0}\left(\frac{t}{T}\right) \mu_{X}\left(\frac{t-l_{r}-b}{T}\right)+\omega_{1}\left(\frac{t}{T}\right) \mu_{X}\left(\frac{t-l_{r}-1-b}{T}\right)+\right.} \\
& \left.+\ldots+\omega_{s}\left(\frac{t}{T}\right) \mu_{X}\left(\frac{t-l_{r}-s-b}{T}\right)\right]+ \\
& +\sum_{l_{r}=0}^{\infty} \sum_{l_{r-1}=0}^{l_{r}} \sum_{l_{r-2}=0}^{l_{r-1}} \ldots \sum_{l_{1}=0}^{l_{2}} \bar{\delta}_{1}^{l_{1}}\left(\frac{t}{T}\right) \bar{\delta}_{2}^{l_{2}-l_{1}}\left(\frac{t}{T}\right) \ldots \bar{\delta}_{r}^{l_{r}-l_{r-1}}\left(\frac{t}{T}\right) \\
& \int_{-\pi}^{\pi}\left[\omega_{0}\left(\frac{t}{T}\right) e^{i \lambda\left(t-l_{r}-b\right)} A_{X}\left(\frac{t-l_{r}-b}{T}, \lambda\right)+\right. \\
& +\omega_{1}\left(\frac{t}{T}\right) e^{i \lambda\left(t-l_{r}-1-b\right)} A_{X}\left(\frac{t-l_{r}-1-b}{T}, \lambda\right)+\ldots+ \\
& \left.+\omega_{s}\left(\frac{t}{T}\right) e^{i \lambda\left(t-l_{r}-s-b\right)} A_{X}\left(\frac{t-l_{r}-s-b}{T}, \lambda\right)\right] d \xi(\lambda),
\end{aligned}
$$

do que resulta

$$
Y_{t, T}=\mu_{Y}\left(\frac{t}{T}\right)+\int_{-\pi}^{\pi} e^{i \lambda t} A_{Y}\left(\frac{t}{T}, \lambda\right) d \xi(\lambda)
$$

com

$$
\begin{aligned}
\mu_{Y}\left(\frac{t}{T}\right)= & \sum_{l_{1}=0}^{\infty} \sum_{l_{2}=0}^{l_{1}} \sum_{l_{3}=0}^{l_{2}} \ldots \sum_{l_{r}=0}^{l_{r-1}} \bar{\delta}_{1}^{l_{1}}\left(\frac{t}{T}\right) \bar{\delta}_{2}^{l_{2}-l_{1}}\left(\frac{t}{T}\right) \ldots \bar{\delta}_{r}^{l_{r}-l_{r-1}}\left(\frac{t}{T}\right) \\
& {\left[\omega_{0}\left(\frac{t}{T}\right) \mu_{X}\left(\frac{t-l_{r}-b}{T}\right)+\omega_{1}\left(\frac{t}{T}\right) \mu_{X}\left(\frac{t-l_{r}-1-b}{T}\right)+\right.} \\
& \left.+\ldots+\omega_{s}\left(\frac{t}{T}\right) \mu_{X}\left(\frac{t-l_{r}-s-b}{T}\right)\right]
\end{aligned}
$$

$\mathrm{e}$

$$
\begin{aligned}
A_{Y}\left(\frac{t}{T}, \lambda\right)= & \sum_{l_{1}=0}^{\infty} \sum_{l_{2}=0}^{l_{1}} \sum_{l_{3}=0}^{l_{2}} \ldots \sum_{l_{r}=0}^{l_{r-1}} \bar{\delta}_{1}^{l_{1}}\left(\frac{t}{T}\right) \bar{\delta}_{2}^{l_{2}-l_{1}}\left(\frac{t}{T}\right) \ldots \bar{\delta}_{r}^{l_{r}-l_{r-1}}\left(\frac{t}{T}\right) \\
& \sum_{m=0}^{s} e^{-i \lambda m} \omega_{m}\left(\frac{t}{T}\right) A_{X}\left(\frac{t-l_{r}-m-b}{T}, \lambda\right) .
\end{aligned}
$$


Vemos que $\mu_{Y}\left(\frac{t}{T}\right)$ e $A_{Y}\left(\frac{t}{T}, \lambda\right)$ são funções de $\mu_{X}\left(\frac{t-l}{T}\right)$ e $A_{X}\left(\frac{t-l}{T}, \lambda\right), l=0,1,2, \ldots$, respectivamente.

Como $\left|\bar{\delta}_{j}\left(\frac{t}{T}\right)\right|<1$, para $j=1,2, \ldots, r$ então $A_{Y}\left(\frac{t-l}{T}, \lambda\right)$ converge para cada $t$. Por outro lado,

$$
\begin{aligned}
\overline{A_{Y}\left(\frac{t}{T}, \lambda\right)=} & \sum_{l_{1}=0}^{\infty} \sum_{l_{2}=0}^{l_{1}} \sum_{l_{3}=0}^{l_{2}} \ldots \sum_{l_{r}=0}^{l_{r-1}} \bar{\delta}_{1}^{l_{1}-l_{2}}\left(\frac{t}{T}\right) \bar{\delta}_{2}^{l_{2}-l_{3}}\left(\frac{t}{T}\right) \ldots \bar{\delta}_{r-1}^{l_{r-1}-l_{r}}\left(\frac{t}{T}\right) \bar{\delta}_{r}^{l_{r-2}}\left(\frac{t}{T}\right) \\
& \sum_{m=0}^{s} e^{i \lambda m} \omega_{m}\left(\frac{t}{T}\right) \overline{A_{X}\left(\frac{t-l_{r}-m-b}{T}, \lambda\right)} \\
= & \sum_{l_{1}=0}^{\infty} \sum_{l_{2}=0}^{l_{1}} \sum_{l_{3}=0}^{l_{2}} \ldots \sum_{l_{r}=0}^{l_{r-1}} \bar{\delta}_{1}^{l_{1}-l_{2}}\left(\frac{t}{T}\right) \bar{\delta}_{2}^{l_{2}-l_{3}}\left(\frac{t}{T}\right) \ldots \bar{\delta}_{r-1}^{l_{r-1}-l_{r}}\left(\frac{t}{T}\right) \bar{\delta}_{r}^{l_{r-2}}\left(\frac{t}{T}\right) \\
& \sum_{m=0}^{s} e^{i \lambda m} \omega_{m}\left(\frac{t}{T}\right) A_{X}\left(\frac{t-l_{r}-m-b}{T},-\lambda\right) \\
= & A_{Y}\left(\frac{t}{T},-\lambda\right) .
\end{aligned}
$$

Mostramos, então, que $Y_{t, T}=\mathrm{v}_{t}(B) X_{t, T}$ é um processo localmente estacionário, segundo Dahlhaus (1997).

Vamos incluir um ruído branco, $\epsilon_{t}$ com média zero e variância $\sigma_{\epsilon}^{2}$, independente da série de entrada $X_{t, T}$ para $t=1,2, \ldots, T$, no modelo (2.3),

$$
Y_{t, T}=\mathrm{v}_{t}(B) X_{t, T}+\epsilon_{t}
$$

com

$$
\epsilon_{t}=\int_{-\pi}^{\pi} e^{i \lambda t} A\left(\frac{t}{T}, \lambda\right) d \zeta(\lambda)
$$

Vemos que $\epsilon_{t}$ é um processo localmente estacionário, $\operatorname{com} \mu\left(\frac{t}{T}\right)=0$ e $A\left(\frac{t}{T}, \lambda\right)=$ $\left(\sigma_{\epsilon}^{2} /(2 \pi)\right)^{-1 / 2}$, para $t=1,2, \ldots, T$. O processo $\zeta(\lambda)$ apresenta as mesmas propriedades de (i) de (2.1) porém é independente de $\xi(\lambda)$, pois o erro é independente da série de entrada. 


\subsection{Ondaletas}

Ondaletas são bases para representar funções. São localizadas no tempo (ou espaço) e são apropriadas para analisar sinais não estacionários. A idéia é aproximar uma função qualquer através de uma combinaçāo linear de pequenas ondas.

A idéia central é considerar pequenas ondas $\psi(x)$ e com elas gerar uma base a partir de dilatações binárias $\left(2^{j}\right)$ e translações diádicas $\left(k / 2^{j}\right)$ da forma

$$
\psi\left(2^{j} x-k\right), \quad j, k \in \mathbb{Z}
$$

Usamos ondaletas para representar funções, $f(x)$, de quadrado integrável, i.e.,

$$
\int_{-\infty}^{\infty}|f(x)|^{2} d x<\infty
$$

Funções geradas a partir de dilatações e translações das funções (2.6) devem ser capazes de representar todas as funções em $L^{2}(\mathbb{R})$. Trabalharemos aqui com bases ortogonais geradas por $\psi$.

Usaremos a notação $\langle f, g\rangle=\int_{-\infty}^{\infty} f(x) \overline{g(x)} d x$ para o produto interno das funções $f$ e $g \in L^{2}(\mathbb{R})$ e $\|f\|_{2}=\langle f, f\rangle^{1 / 2}$ para a norma.

Para quaisquer $j, k \in \mathbb{Z}$, temos

$$
\begin{aligned}
\left\|f\left(2^{j} \cdot-k\right)\right\|_{2} & =\left\{\int_{-\infty}^{\infty}\left|f\left(2^{j} x-k\right)\right|^{2} d x\right\}^{1 / 2} \\
& =2^{-j / 2}\|f\|_{2} .
\end{aligned}
$$

Se tomarmos uma função $\psi \in L^{2}(\mathbb{R})$ de norma unitária, então as funções $\psi_{j k}$ definidas por

$$
\psi_{j k}(x)=2^{j / 2} \psi\left(2^{j} x-k\right) \quad j . k \in \mathbb{Z}
$$


também terão norma unitária,

$$
\left\|\psi_{j k}\right\|_{2}=\|\psi\|=1, \quad j, k \in \mathbb{Z}
$$

A função $\psi \in L^{2}(\mathbb{R})$ é chamada de ondaleta ortogonal se a família $\left\{\psi_{j k}\right\}$, definida em (2.7), é uma base ortonormal de $L^{2}(\mathbb{R})$, i.e., $\left\langle\psi_{j k}, \psi_{l m}\right\rangle=\delta_{j, l} \cdot \delta_{k, m}$ para $j, k, l, m \in \mathbb{Z}$ em que $\delta_{j, l}$ é o delta de Kronecker.

Uma função $f \in L^{2}(\mathbb{R})$ pode ser escrita, nessa nova base, como

$$
f(x)=\sum_{j, k=-\infty}^{\infty} c_{j k} \psi_{j k}(x),
$$

em que $c_{j k}$ são os coeficientes de ondaletas e são dados por $c_{j k}=\left\langle f, \psi_{j k}\right\rangle$.

Seja $W_{j}=$ fecho $_{L^{2}(R)}\left\langle\psi_{j k}: k \in \mathbb{Z}\right\rangle$ o fecho da expansão linear de $\left\{\psi_{j k}: k \in \mathbb{Z}\right\}$ para cada nível $j \in \mathbb{Z}$. Desta forma podemos decompor $L^{2}(\mathbb{R})$ como uma soma direta de espaços $W_{j}$ :

$$
L^{2}(\mathbb{R})=\sum_{j \in \mathbb{Z}} W_{j}=\ldots \oplus W_{-1} \oplus W_{0}+\oplus W_{1} \oplus \ldots
$$

Se fizermos uma decomposição de uma função $f \in L^{2}(\mathbb{R})$ para cada nível teremos, univocamente em $L^{2}(\mathbb{R})$,

$$
f(x)=\ldots+g_{-1}(x)+g_{0}(x)+g_{1}(x)+\ldots
$$

em que $g_{j} \in W_{j}$, para qualquer $j \in \mathbb{Z}$.

Se $\psi$ for uma ondaleta ortogonal, então os subespaços $W_{j}$ de $L^{2}(\mathbb{R})$ são mutuamente ortogonais, ou seja, $W_{j} \perp W_{l}$, para $j \neq l$.

Consideremos agora os subespaços fechados $V_{j}$ de $L^{2}(\mathbb{R})$ definidos como:

$$
V_{j}=\ldots \oplus W_{j-2} \oplus W_{j-1}, \quad j \in \mathbb{Z}
$$


Esses subespaços têm as seguintes propriedades:

1) $\ldots \subset V_{-1} \subset V_{0} \subset V_{1} \subset \ldots$;

2) $\operatorname{fecho}_{L^{2}}\left(\bigcup_{j \in \mathbb{Z}} V_{j}\right)=L^{2}(\mathbb{R})$;

3) $\bigcap_{j \in Z} V_{j}=\{0\}$;

4) $V_{j+1}=V_{j} \oplus W_{j}, \quad j \in Z$;

5) $f(x) \in V_{j} \Leftrightarrow f(2 x) \in V_{j+1}, \quad j \in Z$.

A seqüência de subespaços $V_{j}$ é aninhada. Podemos decompor qualquer função $f \in L^{2}(\mathbb{R})$ em projeções $P_{j} f$ em $V_{j}$, tão próximas quanto o desejável.

Uma propriedade intrínseca e muito importante desses espaços é a retirada de mais e mais variações de $P_{j} f \operatorname{com} j \rightarrow \infty$. As informações são perdidas (detalhe perdido) a cada diminuição de nível $j$. Esses detalhes perdidos são "armazenados" nos subespaços complementares $W_{j}$.

Tomemos agora o subespaço $V_{0}$ como referência, gerado por uma função $\phi \in$ $L^{2}(\mathbb{R})$

$$
V_{0}=\operatorname{fecho}_{L^{2}(R)}\left\langle\phi_{0 k}: k \in \mathbb{Z}\right\rangle
$$

sendo

$$
\phi_{l k}(x)=2^{l / 2} \phi\left(2^{l} x-k\right)
$$

Todos os subespaços $V_{l}$ são também gerados pela mesma $\phi$,

$$
V_{l}=f e c h o_{L^{2}(R)}\left\langle\phi_{l k}: k \in \mathbb{Z}\right\rangle
$$


Assim podemos decompor $V_{l}$ em $W_{l-1}, W_{l-2}, \ldots$ A função $\phi$ é chamada de função escala.

Uma função $f(x) \in \mathbb{R}$ pode, então, ser decomposta da seguinte maneira

$$
f(x)=\sum_{k \in I_{l}^{0}} \alpha_{l k} \phi_{l k}(x)+\sum_{j \geq l} \sum_{k \in I_{j}} \beta_{j k} \psi_{j k}(x),
$$

onde $I_{l}^{0}$ é o conjunto de índices de translações de $\phi, I_{l}^{0}=\left\{0,1,2, \cdots, 2^{l}-1\right\}$ e $I_{j}$ é o conjunto de valores indicando os diferentes coeficientes de ondaletas, $I_{j}=$ $\left\{0,1,2, \cdots, 2^{j}-1\right\}$ associados ao nível $j$ de resolução.

A partir desse ponto, iremos considerar as funções de quadrado integrável com suporte em um intervalo limitado da reta e, sem perda de generalidade, tomaremos o intervalo $(0,1]$.

Desta forma, a função $f \in L^{2}((0,1])$ se

$$
\int_{0}^{1}|f(x)|^{2} d x<\infty
$$

Como as ondaletas são geradas através de translações e dilatações de uma função $(\psi)$, ao trabalharmos com funções com suporte limitado teremos translações e dilatações cujo suporte estará fora do suporte da função. Várias soluções para alteração da base foram propostas e, todas elas preservam a base original na região do suporte que está dentro do suporte da função. Próximo da fronteira a base de ondaletas é modificada.

Ondaletas periódicas é uma solução que apresentamos a seguir; a menos que a função $f(x)$ já seja periódica, as ondaletas periódicas introduzirão uma alteração nas fronteiras da função.

As ondaletas periódicas são definidas como:

$$
\widetilde{\psi}_{j k}(u)=\sum_{n} \psi_{j k}(u-n), \quad j, k \in \mathbb{Z}
$$




$$
\widetilde{\phi}_{l k}(u)=\sum_{n} \phi_{j k}(u-n), \quad l, k \in \mathbb{Z}
$$

sendo $u=t / T, t=1,2, \ldots, T$.

A partir da funções (2.11) construímos a análise de multirresolução.

$$
\widetilde{V}_{0} \subset \widetilde{V}_{1} \subset \widetilde{V}_{2} \subset \cdots
$$

em que $\widetilde{V}_{j}$ é gerado por $\widetilde{\phi}_{j k}$ e, temos ainda,

$$
\widetilde{V}_{j+1}=\widetilde{W}_{j} \oplus \widetilde{V}_{j}
$$

sendo $\widetilde{W}_{j}$ gerado por $\widetilde{\psi}_{j k}$.

A decomposição em ondaletas (2.10) em ondaletas periódicas, tomando $l=0$, será:

$$
f(u)=\alpha_{00} \tilde{\phi}_{00}(u)+\sum_{j \geq 0} \sum_{k \in I_{j}} \beta_{j k} \tilde{\psi}_{j k}(u)
$$

A partir deste ponto sempre usaremos ondaletas periódicas, e a notação $\widetilde{\phi}_{00}(u)$ e $\widetilde{\psi}_{j k}(u)$ será substituída por $\phi_{00}(u)$ e $\psi_{j k}(u)$, respectivamente.

\subsubsection{Transformada de Ondaletas}

Considere um conjunto de observações $\mathrm{X}=\left(X_{0}, X_{1}, \ldots, X_{T-1}\right)^{\prime}, T=2^{J}, J>0$ inteiro. A transformada de ondaletas, na forma matricial, de $\mathbf{X}$ é

$$
\mathrm{d}=\mathrm{WX}
$$

em que

$$
\mathbf{d}=\left[\begin{array}{llllllll}
s_{0} & d_{00} & d_{10} & d_{11} & \cdots & d_{J-1,0} & \cdots & d_{J-1,2^{J-1}-1}
\end{array}\right]^{\prime}
$$


W é uma matriz ortogonal denotada por

$$
\mathbf{W}=\left[\begin{array}{c}
\mathbf{V}_{0} \\
\mathbf{W}_{0} \\
\mathbf{W}_{1} \\
\vdots \\
\mathbf{W}_{J-1}
\end{array}\right]
$$

sendo cada submatriz dada por

$$
\begin{gathered}
\mathbf{V}_{0}=\left[\begin{array}{llll}
\phi_{00}(0) & \phi_{00}(1) & \cdots & \phi_{00}(T-1)
\end{array}\right] \\
\mathbf{W}_{0}=\left[\begin{array}{llll}
\psi_{00}(0) & \psi_{00}(1) & \cdots & \psi_{00}(T-1)
\end{array}\right] \\
\mathbf{W}_{1}=\left[\begin{array}{cccc}
\psi_{10}(0) & \psi_{10}(1) & \cdots & \psi_{10}(T-1) \\
\psi_{11}(0) & \psi_{11}(1) & \cdots & \psi_{11}(T-1)
\end{array}\right], \\
\mathbf{W}_{J-1}=\left[\begin{array}{cccc}
\psi_{J-1,0}(0) & \psi_{J-1,0}(1) & \cdots & \psi_{J-1,0}(T-1) \\
\psi_{J-1,1}(0) & \psi_{J-1,1}(1) & \cdots & \psi_{J-1,1}(T-1) \\
\vdots & \vdots & \ddots & \vdots \\
\psi_{J-1,2^{J-1}-1}(0) & \psi_{J-1,2^{J-1}-1}(1) & \cdots & \psi_{J-1,2^{J-1}-1}(T-1)
\end{array}\right]
\end{gathered}
$$

Assim vemos que $\mathbf{V}_{0}$ e $\mathbf{W}_{0}$ são de ordem 1 por $T, \mathbf{W}_{1}$ de ordem 2 por $T$ e, genericamente $\mathbf{W}_{j}$ de ordem $2^{j}$ por $T$. Como $T=2^{J}$ a matriz $\mathbf{W}$ é de ordem $T$ por $T$. Se tivermos interesse apenas nos coeficientes de ondaletas correspondentes a um determinado nível $j$ fazemos a transformação $\mathbf{d}_{j}=\mathbf{W}_{j} \mathbf{X}, \operatorname{com} j=0,1,2, \cdots, J-1$. Fazendo a transformação inversa de cada $\mathrm{d}_{j}$, isto é $\mathrm{D}_{j}=\mathrm{W}_{j}^{\prime} \mathrm{d}$ teremos cada $\mathrm{D}_{j}$ como detalhe de $\mathbf{X}$ e $\mathbf{s}_{0}=\mathbf{V}_{0} \mathbf{X}$ é chamado parte suave de $\mathbf{X}$. Assim decompomos $\mathrm{X}$ como

$$
\mathbf{X}=\sum_{j=0}^{J} \mathbf{D}_{j}+\mathbf{s}_{0}
$$


Podemos diminuir o nível de resolução da decomposição em ondaletas, isto é, fazemos a decomposição até um nivel $j^{*}$ tal que, $j^{*}<J$. Desta forma a recomposição dessa função não será perfeita, mas, se $j^{*}$ for tal que $2^{j^{*}-1} \leq T^{1 / 2} \leq 2^{j^{*}}$, teremos:

$$
\mathbf{X}=\sum_{j=0}^{j^{*}-1} \mathbf{D}_{j}+\mathbf{s}_{0}+\mathbf{R}_{j^{*}}
$$

$\mathbf{R}_{j^{*}}$ é o ruído referente as escalas mais finas $j^{*}, j^{*}+1, j^{*}+2, \cdots, J$ e $\|\mathbf{X}\|^{2}>>$ $\left\|\mathbf{R}_{j^{*}}\right\|^{2}$.

Construímos $\mathbf{W}$ de maneira que tenhamos um nível de resolução com poucos coeficientes de ondaletas, que levarão a um menor número de parâmetros a serem estimados, porém com uma resolução que nos permita uma representação fidedigna do sinal. Esta técnica é conhecida como Análise de Multirresolução.

Neste trabalho usaremos $l=0$ e $\sharp\left\{I_{l}^{0}\right\}=1$, de modo que (2.10) fica

$$
f(u)=\alpha_{00} \phi_{00}(u)+\sum_{j \geq 0} \sum_{k \in I_{j}} \beta_{j k} \psi_{j k}(u)
$$

Supondo decomposição até o nível $j^{*}$,

$$
f(u) \simeq \alpha_{00} \phi_{00}(u)+\sum_{j=0}^{j^{*}-1} \sum_{k \in I_{j}} \beta_{j k} \psi_{j k}(u) .
$$

Como $\phi_{00} \cup\left\{\psi_{j k}\right\}_{0 \leq j \leq j^{*}-1 ; k}$ forma uma base ortonormal de $L^{2}((0,1])$, teremos uma aproximação de $f$ apenas no espaço $V^{j^{*}}$.

A técnica denominada limiarização (thresholding) consiste em reduzir o ruído presente em um sinal, através da diminuição (limiarização suave) ou eliminação (limiarização dura) dos coeficientes de ondaletas $\beta_{j k}$. A limiarização suave é realizada ao aplicarmos a função $\delta^{(s)}$ e, na limiarização dura, aplicamos a função $\delta^{(h)}$, dadas respectivamente por

$$
\begin{aligned}
& \delta^{(s)}\left(\hat{\beta}_{j k}, \lambda\right)=\left(\left|\hat{\beta}_{j k}\right|-\lambda\right)_{+} \operatorname{sinal}\left(\hat{\beta}_{j k}\right), \\
& \delta^{(h)}\left(\hat{\beta}_{j k}, \lambda\right)=\hat{\beta}_{j k} I\left(\left|\hat{\beta}_{j k}\right| \geq \lambda\right),
\end{aligned}
$$


onde $\hat{\beta}_{j k}, j \geq 0,2^{j} \leq T^{1 / 2}, k \in I_{j}$, são os coeficientes empíricos de ondaleta da função $f$, obtido por algum procedimento de estimação.

No caso em que os coeficientes empíricos de ondaletas forem normais, $\hat{\beta}_{j k}^{(f)} \sim$ $\mathcal{N}\left(\beta_{j k}, \sigma^{2}\right)$, um método muito popular é aplicar limiares $\lambda=\sigma \sqrt{2 \log n}$, com $n$ sendo o número de coeficientes e $\sigma^{2}$ a variância do ruído. Este limiar é chamado limiar universal. Outros procedimentos são o limiar adaptativo (SureShrink), o limiar de validação cruzada e uma variante do limiar universal proposta por Donoho, $\operatorname{com} \lambda=\sigma \sqrt{\frac{2 \log n}{T}}$. Mais detalhes sobre esse limiares podem ser encontrados em, por exemplo, Percival e Walden (2000) e Morettin (1999). Quanto a estimativa de $\sigma$, podemos optar por calcular um único valor, i.e., um valor englobando todos os níveis, ou um valor para cada nível $j$ ou, ainda, utilizar apenas o ruído relativo ao nível mais fino da decomposição em ondaletas. 


\section{Capítulo 3}

\section{Estimação do Modelo de Função de Transferência}

\subsection{Estimadores Propostos}

O modelo que será tratado neste trabalho é

$$
Y_{t, T}=\frac{\boldsymbol{\omega}_{t}(B) B^{b}}{\boldsymbol{\delta}_{t}(B)} X_{t, T}+\epsilon_{t}
$$

$\operatorname{com} \epsilon_{t}$ independentes e identicamente distribuídos com média zero e variância 1, e

$$
\frac{\boldsymbol{\omega}_{t}(B)}{\delta_{t}(B)}=\frac{\omega_{0}(t)+\omega_{1}(t) B+\omega_{2}(t) B^{2}+\ldots+\omega_{s}(t) B^{s}}{1-\delta_{1}(t) B-\delta_{2}(t) B^{2}-\ldots-\delta_{r}(t) B^{r}} .
$$

Definidos $b$ e as ordens $r$ e $s$ dos polinômios $\delta_{t}(B)$ e $\omega_{t}(B)$, respectivamente, o próximo passo é a estimação dos coeficientes desses dois polinômios usando a decomposição em ondaletas de cada um desses coeficientes. A ídeia é encontrar os coeficientes de ondaletas empíricos, como em Dahlhaus et al. (1999) e depois 
reconstruir as referidas funções. Estimamos, neste trabalho, os coeficientes de ondaletas empíricos através de mínimos quadrados, em dois estágios e verificamos que os coeficientes do primeiro estágio têm distribuição aproximadamente normal.

A estimação proposta aqui é realizada em dois estágios, que passamos a descrever. Considere o modelo (2.5) e a situação em que $b=0, r=1$ e $s=0$, ou seja,

$$
Y_{t, T}=\frac{\omega_{0}\left(\frac{t}{T}\right)}{1-\delta_{1}\left(\frac{t}{T}\right) B} X_{t, T}+\epsilon_{t}
$$

No primeiro estágio ajustamos o modelo de regressão de $Y_{t, T}$ (variável resposta) sobre $X_{t, T}$ e $Y_{t-1, T}$ (variáveis explicativas), ou seja,

$$
Y_{t, T}=\delta_{1}(t / T) Y_{t-1, T}+\omega_{0}(t / T) X_{t, T}+e_{t, T}
$$

$e_{t, T}$ é o erro do modelo de regressão do primeiro estágio. Substituindo as funções $\delta_{1}(t / T)$ e $\omega_{0}(t / T)$ pelas correspondentes decomposições em ondaletas dadas em (2.15), obtemos

$$
\begin{aligned}
Y_{t, T}= & \left(\alpha_{00}^{\left(\delta_{1}\right)} \phi_{00}\left(\frac{t}{T}\right)+\sum_{j} \sum_{k} \beta_{j k}^{\left(\delta_{1}\right)} \psi_{j k}\left(\frac{t}{T}\right)\right) Y_{t-1, T}+ \\
& +\left(\alpha_{00}^{\left(\omega_{0}\right)} \phi_{00}\left(\frac{t}{T}\right)+\sum_{j} \sum_{k} \beta_{j k}^{\left(\omega_{0}\right)} \psi_{j k}\left(\frac{t}{T}\right)\right) X_{t, T}+e_{t, T}
\end{aligned}
$$

ou seja,

$$
\begin{aligned}
Y_{t, T}= & \alpha_{00}^{\left(\delta_{1}\right)}\left[\phi_{00}\left(\frac{t}{T}\right) Y_{t-1, T}\right]+\sum_{j} \sum_{k} \beta_{j k}^{\left(\delta_{1}\right)}\left[\psi_{j k}\left(\frac{t}{T}\right) Y_{t-1, T}\right]+ \\
& +\alpha_{00}^{\left(\omega_{0}\right)}\left[\phi_{00}\left(\frac{t}{T}\right) X_{t, T}\right]+\sum_{j} \sum_{k} \beta_{j k}^{\left(\omega_{0}\right)}\left[\psi_{j k}\left(\frac{t}{T}\right) X_{t, T}\right]+e_{t, T}
\end{aligned}
$$

Os valores de $j$ e $k$ dependem do nível de resolução desejado da decomposição de ondaletas. 
Genericamente usaríamos $s+b$ defasagens de $X_{t, T}$ e $r$ defasagens de $Y_{t, T}$.

De posse das estimativas dos coeficientes usamos a transformada inversa de ondaletas para encontrar as estimativas $\hat{\omega}_{0}\left(\frac{t}{T}\right)$ e $\hat{\delta}_{1}\left(\frac{t}{T}\right)$ das funções $\omega_{0}\left(\frac{t}{T}\right)$ e $\delta_{1}\left(\frac{t}{T}\right)$, respectivamente. Podemos, também, fazer uma limiarização dos coeficientes de ondaletas, seguido da transformada inversa de ondaletas, obtendo assim as estimativas limiarizada $\hat{\delta}_{1}^{(L)}\left(\frac{t}{T}\right)$ e $\hat{\omega}_{0}^{(L)}\left(\frac{t}{T}\right)$ das funções $\delta_{1}^{(L)}\left(\frac{t}{T}\right)$ e $\omega_{1}^{(L)}\left(\frac{t}{T}\right)$.

A seguir, estimamos o valor de $Y_{t, T}$ por

$$
\begin{aligned}
\hat{Y}_{t, T} & =\hat{\omega}_{0}^{(L)}\left(\frac{t}{T}\right) X_{t, T}+\hat{\delta}_{1}^{(L)}\left(\frac{t}{T}\right) B Y_{t, T} \\
& =\hat{\omega}_{0}^{(L)}\left(\frac{t}{T}\right) X_{t, T}+\hat{\delta}_{0}^{(L)}\left(\frac{t}{T}\right) Y_{t-1, T}
\end{aligned}
$$

No segundo estágio fazemos uma regressão de $Y_{t, T}$ (variável resposta) sobre $X_{t, T}$ e $\hat{Y}_{t-1, T}$ (variáveis explicativas).

As estimativas finais $\tilde{\omega}_{0}\left(\frac{t}{T}\right)$ e $\tilde{\delta}_{1}\left(\frac{t}{T}\right)$ das funções $\omega_{0}\left(\frac{t}{T}\right)$ e $\delta_{1}\left(\frac{t}{T}\right)$ são encontradas através da transformada inversa de ondaletas e novamente obtemos as funções $\tilde{\omega}_{0}^{(L)}\left(\frac{t}{T}\right)$ e $\tilde{\delta}_{1}^{(L)}\left(\frac{t}{T}\right)$ através da transformada inversa de ondaletas após a limiarização dos coeficientes estimados.

Esse procedimento é justificado pelo que segue. O modelo é

$$
\begin{aligned}
Y_{t, T} & =\frac{\omega_{0}\left(\frac{t}{T}\right)}{1-\delta_{1}\left(\frac{t}{T}\right) B} X_{t, T}+\epsilon_{t} \\
{\left[1-\delta_{1}\left(\frac{t}{T}\right) B\right] Y_{t, T} } & =\omega_{0}\left(\frac{t}{T}\right) X_{t, T}+\left[1-\delta_{1}\left(\frac{t}{T}\right) B\right] \epsilon_{t} \\
Y_{t, T} & =\delta_{1}\left(\frac{t}{T}\right) Y_{t-1, T}+\omega_{0}\left(\frac{t}{T}\right) X_{t, T}+\epsilon_{t}-\delta_{1}\left(\frac{t}{T}\right) \epsilon_{t-1}, \\
Y_{t, T} & =\delta_{1}\left(\frac{t}{T}\right)\left[Y_{t-1, T}-\epsilon_{t-1}\right]+\omega_{0}\left(\frac{t}{T}\right) X_{t, T}+\epsilon_{t} .
\end{aligned}
$$

Como desconhecemos os erros, fazemos uma regressão de mínimos quadrados de $Y_{t, T}$ sobre $Y_{t-1, T}$ e $X_{t, T}$ para encontrar os coeficientes de ondaletas e reconstruir 
$\hat{\delta}_{1}^{(L)}\left(\frac{t}{T}\right)$ e $\hat{\omega}_{0}^{(L)}\left(\frac{t}{T}\right)$ e, com essas estimativas, determinamos $\hat{Y}_{t, T}$ por

$$
\hat{Y}_{t, T}=\hat{\delta}_{1}^{(L)}\left(\frac{t}{T}\right) Y_{t-1, T}+\omega_{0}^{(L)}\left(\frac{t}{T}\right) X_{t, T}
$$

Calculamos $\bar{e}_{t, T}$ como

$$
\bar{e}_{t, T}=Y_{t, T}-\hat{Y}_{t, T}
$$

No segundo estágio, substituímos $\epsilon_{t}$ por $\bar{e}_{t, T}$ em (3.2),

$$
Y_{t, T}=\delta_{1}\left(\frac{t}{T}\right)\left[Y_{t-1, T}-\bar{e}_{t-1, T}\right]+\omega_{0}\left(\frac{t}{T}\right) X_{t, T}+\eta_{t, T},
$$

e como $Y_{t, T}-\bar{e}_{t, T}=\hat{Y}_{t, T}$ para todo $t$, ajustamos a regressão de $Y_{t, T}$ sobre $\hat{Y}_{t-1, T}$ e $X_{t, T}$

$$
Y_{t, T}=\delta_{1}\left(\frac{t}{T}\right) \hat{Y}_{t-1, T}+\omega_{0}\left(\frac{t}{T}\right) X_{t, T}+\eta_{t, T}
$$

para encontrar $\tilde{\delta}_{1}^{(L)}\left(\frac{t}{T}\right)$ e $\tilde{\omega}_{0}^{(L)}\left(\frac{t}{T}\right)$ no segundo estágio. As estimativas dos coeficientes dessa nova regressão são as estimativas finais.

\subsection{Estimadores do Primeiro Estágio}

Consideremos o modelo de função de transferência

$$
Y_{t, T}=\frac{\omega_{0}\left(\frac{t}{T}\right)}{1-\delta_{1}\left(\frac{t}{T}\right) B} X_{t, T}+\epsilon_{t}
$$

Para encontrarmos $\hat{\delta}_{1}\left(\frac{t}{T}\right)$ e $\hat{\omega}_{0}\left(\frac{t}{T}\right)$, consideramos a regressão,

$$
Y_{t, T}=\delta_{1}\left(\frac{t}{T}\right) Y_{t-1, T}+\omega_{0}\left(\frac{t}{T}\right) X_{t, T}+e_{t, T}
$$


Usando a decomposição de ondaletas descrita anteriormente, truncada no nível $j^{*}$, temos

$$
\begin{aligned}
Y_{t, T}= & {\left[\alpha_{00}^{\left(\delta_{1}\right)} \phi_{00}\left(\frac{t}{T}\right)+\sum_{j=0}^{j^{*}-1} \sum_{k \in I_{j}} \beta_{j k}^{\left(\delta_{1}\right)} \psi_{j k}\left(\frac{t}{T}\right)\right] Y_{t-1, T}+} \\
& +\left[\alpha_{00}^{\left(\omega_{0}\right)} \phi_{00}\left(\frac{t}{T}\right)+\sum_{j=0}^{j *-1} \sum_{k \in I_{j}} \beta_{j k}^{\left(\omega_{0}\right)} \psi_{j k}\left(\frac{t}{T}\right)\right] X_{t, T}+e_{t, T}
\end{aligned}
$$

$t=2,3,4, \ldots, T, I_{l}^{o}=\{0\}, I_{j}=\left\{0,1,2, \cdots, 2^{j}-1\right\}, j^{*}$ definido anteriormente. Para podermos reduzir a notação, denotaremos $\Delta=2^{j^{*}-1}-1$.

Em notação matricial, temos,

$$
\begin{gathered}
{\left[\begin{array}{c}
Y_{2, T} \\
Y_{3, T} \\
\vdots \\
Y_{T, T}
\end{array}\right]=\left[\begin{array}{cccc}
\phi_{00}\left(\frac{2}{T}\right) Y_{1, T} & \psi_{00}\left(\frac{2}{T}\right) Y_{1, T} & \cdots & \psi_{j^{*}-1, \Delta}\left(\frac{2}{T}\right) Y_{1, T} \\
\phi_{00}\left(\frac{3}{T}\right) Y_{2, T} & \psi_{00}\left(\frac{3}{T}\right) Y_{2, T} & \cdots & \psi_{j^{*}-1, \Delta}\left(\frac{3}{T}\right) Y_{2, T} \\
\vdots & \vdots & \ddots & \vdots \\
\phi_{00}\left(\frac{T}{T}\right) Y_{T-1, T} & \psi_{00}\left(\frac{T}{T}\right) Y_{T-1, T} & \cdots & \psi_{j^{*}-1, \Delta}\left(\frac{T}{T}\right) Y_{T-1, T}
\end{array}\right]\left[\begin{array}{c}
\alpha_{00}^{\delta_{1}} \\
\beta_{00}^{\delta_{1}} \\
\vdots \\
\beta_{j^{*}-1, \Delta}^{\delta_{1}}
\end{array}\right]+} \\
+\left[\begin{array}{ccccc}
\phi_{00}\left(\frac{2}{T}\right) X_{2, T} & \psi_{00}\left(\frac{2}{T}\right) X_{2, T} & \cdots & \psi_{j^{*-1}-\Delta}\left(\frac{2}{T}\right) X_{2, T} \\
\phi_{00}\left(\frac{3}{T}\right) X_{3, T} & \psi_{00}\left(\frac{3}{T}\right) X_{3, T} & \cdots & \psi_{j^{*}-1, \Delta}\left(\frac{3}{T}\right) X_{3, T} \\
\vdots & \vdots & \ddots & \vdots \\
\alpha_{00}^{\omega_{0}} \\
\beta_{00}^{\omega_{0}} \\
\vdots \\
\phi_{00}^{\omega_{0}}\left(\frac{T}{T}\right) X_{T, T} & \psi_{00}\left(\frac{T}{T}\right) X_{T, T} & \cdots & \psi_{j^{*}-1, \Delta}\left(\frac{T}{T}\right) X_{T, T}
\end{array}\right]+\left[\begin{array}{c}
e_{2, T} \\
e_{3, T} \\
\vdots \\
e_{T, T}
\end{array}\right]
\end{gathered}
$$

Consideremos as matrizes

$$
\Phi_{Y_{(-1)}}=\left[\begin{array}{c}
\phi_{00}\left(\frac{2}{T}\right) Y_{1, T} \\
\phi_{00}\left(\frac{3}{T}\right) Y_{2, T} \\
\vdots \\
\phi_{00}\left(\frac{T}{T}\right) Y_{T-1, T}
\end{array}\right]
$$

$$
\Psi_{Y_{(-1)}}^{(0)}=\left[\begin{array}{c}
\psi_{00}\left(\frac{2}{T}\right) Y_{1, T} \\
\psi_{00}\left(\frac{3}{T}\right) Y_{2, T} \\
\vdots \\
\psi_{00}\left(\frac{T}{T}\right) Y_{T-1, T}
\end{array}\right], \quad \Psi_{Y_{(-1)}}^{(1)}=\left[\begin{array}{cc}
\psi_{10}\left(\frac{2}{T}\right) Y_{1, T} & \psi_{11}\left(\frac{2}{T}\right) Y_{1, T} \\
\psi_{10}\left(\frac{3}{T}\right) Y_{2, T} & \psi_{11}\left(\frac{3}{T}\right) Y_{2, T} \\
\vdots & \vdots \\
\psi_{10}\left(\frac{T}{T}\right) Y_{T-1, T} & \psi_{11}\left(\frac{T}{T}\right) Y_{T-1, T}
\end{array}\right]
$$


e, genericamente, para $0 \leq m \leq j^{*}-1$,

$$
\Psi_{Y_{(-1)}^{(m)}}^{(m)}=\left[\begin{array}{cccc}
\psi_{m 0}\left(\frac{2}{T}\right) Y_{1, T} & \psi_{m 1}\left(\frac{2}{T}\right) Y_{1, T} & \cdots & \psi_{m, 2^{m}-1}\left(\frac{2}{T}\right) Y_{1, T} \\
\psi_{m 0}\left(\frac{3}{T}\right) Y_{2, T} & \psi_{m 1}\left(\frac{3}{T}\right) Y_{2, T} & \cdots & \psi_{m, 2^{m}-1}\left(\frac{3}{T}\right) Y_{2, T} \\
\vdots & \vdots & \ddots & \vdots \\
\psi_{m 0}\left(\frac{T}{T}\right) Y_{T-1, T} & \psi_{m 1}\left(\frac{T}{T}\right) Y_{T-1, T} & \cdots & \psi_{m, 2^{m}-1}\left(\frac{T}{T}\right) Y_{T-1, T}
\end{array}\right]
$$

O sub-índice $(-1)$ indica defasagem um entre o argumento $t$ da função $\psi\left(\frac{t}{T}\right)$ e o índice da série. Consideremos, também,

$$
\begin{gathered}
\Phi_{X}=\left[\begin{array}{c}
\phi_{00}\left(\frac{2}{T}\right) X_{2, T} \\
\phi_{00}\left(\frac{3}{T}\right) X_{3, T} \\
\vdots \\
\phi_{00}\left(\frac{T}{T}\right) X_{T, T}
\end{array}\right] \\
\Psi_{X}^{(0)}=\left[\begin{array}{c}
\psi_{00}\left(\frac{2}{T}\right) X_{2, T} \\
\psi_{00}\left(\frac{3}{T}\right) X_{3, T} \\
\vdots \\
\psi_{00}\left(\frac{T}{T}\right) X_{T, T}
\end{array}\right], \Psi_{X}^{(1)}=\left[\begin{array}{cc}
\psi_{01}\left(\frac{2}{T}\right) X_{2, T} & \psi_{11}\left(\frac{2}{T}\right) X_{2, T} \\
\psi_{01}\left(\frac{3}{T}\right) X_{3, T} & \psi_{11}\left(\frac{3}{T}\right) X_{3, T} \\
\vdots & \vdots \\
\psi_{01}\left(\frac{T}{T}\right) X_{T, T} & \psi_{11}\left(\frac{T}{T}\right) X_{T, T}
\end{array}\right]
\end{gathered}
$$

e, genericamente, para $0 \leq m \leq j^{*}-1$,

$$
\Psi_{X}^{(m)}=\left[\begin{array}{cccc}
\psi_{m 0}\left(\frac{2}{T}\right) X_{2, T} & \psi_{m 1}\left(\frac{2}{T}\right) X_{2, T} & \cdots & \psi_{m, 2^{m}-1}\left(\frac{2}{T}\right) X_{2, T} \\
\psi_{m 0}\left(\frac{3}{T}\right) X_{3, T} & \psi_{m 1}\left(\frac{3}{T}\right) X_{3, T} & \cdots & \psi_{m, 2^{m}-1}\left(\frac{3}{T}\right) X_{3, T} \\
\vdots & \vdots & \ddots & \vdots \\
\psi_{m 0}\left(\frac{T}{T}\right) X_{T, T} & \dot{\psi}_{m 1}\left(\frac{T}{T}\right) X_{T, T} & \cdots & \dot{\psi}_{m, 2^{m}-1}\left(\frac{T}{T}\right) X_{T, T}
\end{array}\right]
$$

Então, podemos escrever

$$
\begin{aligned}
\mathbf{Y}= & {\left[\begin{array}{lllll}
\Phi_{Y_{(-1)}} & \Psi_{Y_{(-1)}^{(0)}}^{\left(\Psi_{Y_{(-1)}}^{(1)}\right.} & \cdots & \Psi_{Y_{(-1)}^{\left(j^{*}-1\right)}}^{\left(j^{\prime}\right.}
\end{array}\right] \boldsymbol{\beta}^{\left(\delta_{1}\right)}+} \\
& +\left[\begin{array}{lllll}
\Phi_{X} & \Psi_{X}^{(0)} & \Psi_{X}^{(1)} & \ldots & \Psi_{X}^{\left(j^{*}-1\right)}
\end{array}\right] \boldsymbol{\beta}^{\left(\omega_{0}\right)}+\mathbf{e}
\end{aligned}
$$


em que

$$
\begin{aligned}
& \mathbf{Y}=\left(\begin{array}{llll}
\mathbf{Y}_{2, T} & \mathbf{Y}_{3, T} & \cdots & \mathbf{Y}_{T, T}
\end{array}\right)^{\prime} \\
& \boldsymbol{\beta}^{\left(\delta_{1}\right)}=\left(\begin{array}{llllllll}
\alpha_{00}^{\left(\delta_{1}\right)} & \beta_{00}^{\left(\delta_{1}\right)} & \beta_{10}^{\left(\delta_{1}\right)} & \beta_{11}^{\left(\delta_{1}\right)} & \cdots & \beta_{j^{*}-1,0}^{\left(\delta_{1}\right)} & \cdots & \beta_{j^{*}-1, \Delta}^{\left(\delta_{1}\right)}
\end{array}\right)^{\prime}
\end{aligned}
$$

e

$$
\boldsymbol{\beta}^{\left(\omega_{0}\right)}=\left(\begin{array}{llllllll}
\alpha_{00}^{\left(\omega_{0}\right)} & \beta_{00}^{\left(\omega_{0}\right)} & \beta_{10}^{\left(\omega_{0}\right)} & \beta_{11}^{\left(\omega_{0}\right)} & \cdots & \beta_{j^{*}-1,0}^{\left(\omega_{0}\right)} & \cdots & \beta_{j^{*}-1, \Delta}^{\left(\omega_{0}\right)}
\end{array}\right)^{\prime}
$$

Ou ainda,

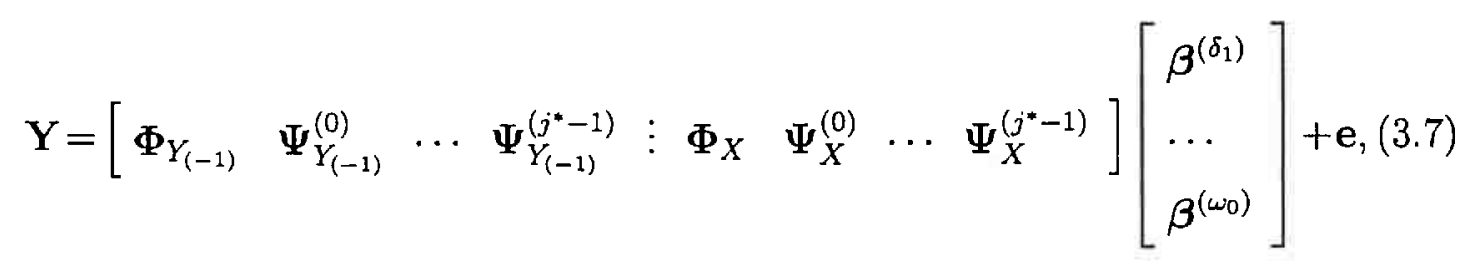

de onde temos

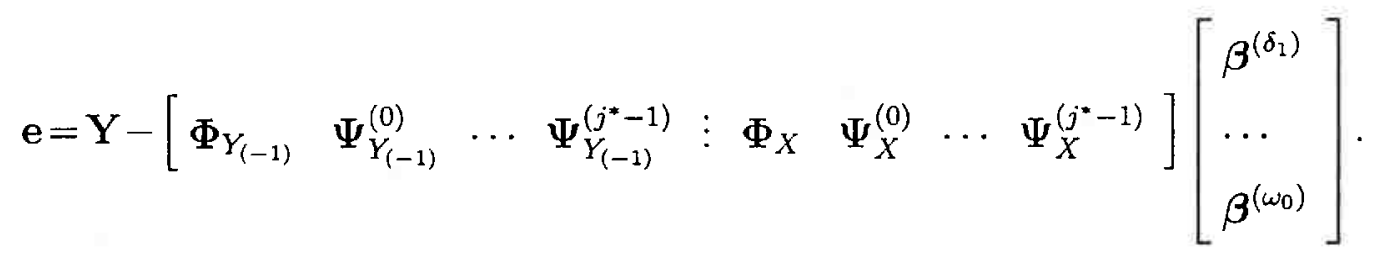

Chamando

$$
f\left(\beta^{\left(\delta_{1}\right)}, \boldsymbol{\beta}^{\left(\omega_{0}\right)}\right)=\mathbf{e}^{\prime} \mathbf{e}
$$

o estimador de mínimos quadrados de $\left(\begin{array}{c}\boldsymbol{\beta}^{\left(\delta_{1}\right)} \\ \boldsymbol{\beta}^{\left(\omega_{0}\right)}\end{array}\right)$ é o valor $\left(\begin{array}{c}\hat{\boldsymbol{\beta}}^{\left(\delta_{1}\right)} \\ \left.\hat{\boldsymbol{\beta}}^{\left(\omega_{0}\right)}\right)\end{array}\right)$ que minimiza a equação (3.9) com e dado por (3.8).

Desta forma, (3.9) pode ser escrita como

$$
f\left(\boldsymbol{\beta}^{\left(\delta_{1}\right)}, \boldsymbol{\beta}^{\left(\omega_{0}\right)}\right)=\left[\mathbf{Y}-\left(\Psi_{Y_{(-1)}} \Psi_{X}\right)\left(\begin{array}{c}
\boldsymbol{\beta}^{\left(\delta_{1}\right)} \\
\boldsymbol{\beta}^{\left(\omega_{0}\right)}
\end{array}\right)\right]^{\prime}\left[\mathbf{Y}-\left(\Psi_{Y_{(-1)}} \Psi_{X}\right)\left(\begin{array}{c}
\boldsymbol{\beta}^{\left(\delta_{1}\right)} \\
\boldsymbol{\beta}^{\left(\omega_{0}\right)}
\end{array}\right)\right]
$$

em que

$$
\Psi_{Y_{(-1)}}=\left[\begin{array}{llll}
\Phi_{Y_{(-1)}} & \Psi_{Y_{(-1)}}^{(0)} & \cdots & \Psi_{Y_{(-1)}}^{\left(j^{*}-1\right)}
\end{array}\right]
$$


e

$$
\Psi_{X}=\left[\begin{array}{llll}
\Phi_{X} & \Psi_{X}^{(0)} & \cdots & \Psi_{X}^{\left(j^{*}-1\right)}
\end{array}\right]
$$

Assim

$$
\begin{aligned}
f\left(\boldsymbol{\beta}^{\left(\delta_{1}\right)}, \boldsymbol{\beta}^{\left(\omega_{0}\right)}\right)= & \mathbf{Y}^{\prime} \mathbf{Y}-\mathbf{Y}^{\prime}\left(\Psi_{Y_{(-1)}} \boldsymbol{\beta}^{\left(\delta_{1}\right)}\right)-\mathbf{Y}^{\prime}\left(\Psi_{X} \boldsymbol{\beta}^{\left(\omega_{0}\right)}\right)-\left(\Psi_{Y_{(-1)}} \boldsymbol{\beta}^{\left(\delta_{1}\right)}\right)^{\prime} \mathbf{Y}+ \\
& -\left(\Psi_{X} \boldsymbol{\beta}^{\left(\omega_{0}\right)}\right)^{\prime} \mathbf{Y}+\left(\Psi_{Y_{(-1)}} \boldsymbol{\beta}^{\left(\delta_{1}\right)}\right)^{\prime}\left(\Psi_{Y_{(-1)}} \boldsymbol{\beta}^{\left(\delta_{1}\right)}\right)+ \\
& +\left(\Psi_{Y_{(-1)}} \boldsymbol{\beta}^{\left(\delta_{1}\right)}\right)^{\prime}\left(\Psi_{X} \boldsymbol{\beta}^{\left(\omega_{0}\right)}\right)+\left(\Psi_{X} \boldsymbol{\beta}^{\left(\omega_{0}\right)}\right)^{\prime}\left(\Psi_{Y_{(-1)}} \boldsymbol{\beta}^{\left(\delta_{1}\right)}\right)+ \\
& +\left(\Psi_{X} \boldsymbol{\beta}^{\left(\omega_{0}\right)}\right)^{\prime}\left(\Psi_{X} \boldsymbol{\beta}^{\left(\omega_{0}\right)}\right) .
\end{aligned}
$$

Calculando as derivadas,

$$
\begin{aligned}
\frac{\partial f\left(\boldsymbol{\beta}^{\left(\delta_{1}\right)}, \boldsymbol{\beta}^{\left(\omega_{0}\right)}\right)}{\partial \boldsymbol{\beta}^{\left(\delta_{1}\right)}=} & -\left(\mathbf{Y}^{\prime} \Psi_{Y_{(-1)}}\right)^{\prime}+\left(\boldsymbol{\beta}^{\left(\delta_{1}\right)^{\prime}} \Psi_{Y_{(-1)}^{\prime}}^{\prime} \Psi_{Y_{(-1)}}\right)^{\prime}+\left(\boldsymbol{\beta}^{\left(\omega_{0}\right)^{\prime}} \Psi_{X}^{\prime} \Psi_{Y_{(-1)}}\right)^{\prime} \\
= & -\Psi_{Y_{(-1)}^{\prime}}^{\prime} \mathbf{Y}-\Psi_{Y_{(-1)}}^{\prime} \Psi_{Y_{(-1)}} \boldsymbol{\beta}^{\left(\delta_{1}\right)}+\Psi_{Y_{(-1)}}^{\prime} \Psi_{X} \boldsymbol{\beta}^{\left(\omega_{0}\right)} \\
\frac{\partial f\left(\boldsymbol{\beta}^{\left(\delta_{1}\right)}, \boldsymbol{\beta}^{\left(\omega_{0}\right)}\right)}{\partial \boldsymbol{\beta}^{\left(\omega_{0}\right)}}= & -\left(\mathbf{Y}^{\prime} \Psi_{X}\right)^{\prime}+\left(\boldsymbol{\beta}^{\left(\delta_{1}\right)^{\prime}} \Psi_{Y_{(-1)}}^{\prime} \Psi_{X}\right)^{\prime}+\left(\boldsymbol{\beta}^{\left(\omega_{0}\right)^{\prime}} \Psi_{X}^{\prime} \Psi_{X}\right)^{\prime} \\
= & -\Psi_{X}^{\prime} \mathbf{Y}+\Psi_{X}^{\prime} \Psi_{Y_{(-1)}} \boldsymbol{\beta}^{\left(\delta_{1}\right)}+\Psi_{X}^{\prime} \Psi_{X} \boldsymbol{\beta}^{\left(\omega_{0}\right)} \\
& \frac{\partial^{2} f\left(\boldsymbol{\beta}^{\left(\delta_{1}\right)}, \boldsymbol{\beta}^{\left(\omega_{0}\right)}\right)}{\partial \boldsymbol{\beta}^{\left(\delta_{1}\right)} \partial\left(\boldsymbol{\beta}^{\left(\delta_{1}\right)}\right)^{\prime}}=-\Psi_{Y_{(-1)}}^{\prime} \Psi_{\left.Y_{(-1)}\right)} \\
& \frac{\partial^{2} f\left(\boldsymbol{\beta}^{\left(\delta_{1}\right)}, \boldsymbol{\beta}^{\left(\omega_{0}\right)}\right)}{\partial \boldsymbol{\beta}^{\left(\omega_{0}\right)} \partial\left(\boldsymbol{\beta}^{\left(\omega_{0}\right)}\right)^{\prime}}=-\Psi_{X}^{\prime} \Psi_{X} \\
& \frac{\partial^{2} f\left(\boldsymbol{\beta}^{\left(\delta_{1}\right)}, \boldsymbol{\beta}^{\left(\omega_{0}\right)}\right)}{\partial \boldsymbol{\beta}^{\left(\delta_{1}\right)} \partial\left(\boldsymbol{\beta}^{\left(\omega_{0}\right)}\right)^{\prime}}= \\
& \frac{\partial^{2} f\left(\boldsymbol{\beta}^{\left(\delta_{1}\right)}, \boldsymbol{\beta}^{\left(\omega_{0}\right)}\right)}{\partial \boldsymbol{\beta}^{\left(\omega_{0}\right)} \partial\left(\boldsymbol{\beta}^{\left(\delta_{1}\right)}\right)^{\prime}}=-\Psi_{Y_{(-1)}}^{\prime} \Psi_{X} \\
& =-\Psi_{X}^{\prime} \Psi_{Y_{(-1)}}
\end{aligned}
$$

e igualando a zero as primeiras derivadas, obtemos as equações

$$
\begin{aligned}
\Psi_{Y_{(-1)}}^{\prime} \mathbf{Y} & =\Psi_{Y_{(-1)}}^{\prime} \Psi_{Y_{(-1)}} \hat{\boldsymbol{\beta}}^{\left(\delta_{1}\right)}+\Psi_{Y_{(-1)}}^{\prime} \Psi_{X} \hat{\boldsymbol{\beta}}^{\left(\omega_{0}\right)} \\
\Psi_{X}^{\prime} \mathbf{Y} & =\Psi_{X}^{\prime} \Psi_{Y_{i-1)}} \hat{\boldsymbol{\beta}}^{\left(\delta_{1}\right)}+\Psi_{X}^{\prime} \Psi_{X} \hat{\boldsymbol{\beta}}^{\left(\omega_{0}\right)},
\end{aligned}
$$


isto é,

$$
\left[\begin{array}{l}
\Psi_{Y_{(-1)}}^{\prime} \mathbf{Y} \\
\Psi_{X}^{\prime} \mathbf{Y}
\end{array}\right]=\left[\begin{array}{ll}
\Psi_{Y_{(-1)}}^{\prime} \Psi_{Y_{(-1)}} & \Psi_{Y_{(-1)}}^{\prime} \Psi_{X} \\
\Psi_{X}^{\prime} \Psi_{Y_{(-1)}} & \Psi_{X}^{\prime} \Psi_{X}
\end{array}\right]\left[\begin{array}{l}
\hat{\boldsymbol{\beta}}^{\left(\delta_{1}\right)} \\
\hat{\boldsymbol{\beta}}^{\left(\omega_{0}\right)}
\end{array}\right]
$$

Então, o estimador de mínimos quadrados é dado por

$$
\left[\begin{array}{l}
\hat{\boldsymbol{\beta}}^{\left(\delta_{1}\right)} \\
\hat{\boldsymbol{\beta}}^{\left(\omega_{0}\right)}
\end{array}\right]=\left[\begin{array}{ll}
\Psi_{Y_{(-1)}}^{\prime} \Psi_{Y_{(-1)}} & \Psi_{Y_{(-1)}}^{\prime} \Psi_{X} \\
\Psi_{X}^{\prime} \Psi_{Y_{(-1)}} & \Psi_{X}^{\prime} \Psi_{X}
\end{array}\right]^{-1}\left[\begin{array}{l}
\Psi_{Y_{(-1)}}^{\prime} \mathbf{Y} \\
\Psi_{X}^{\prime} \mathbf{Y}
\end{array}\right]
$$

Note que:

$$
\Psi_{Y_{(-1)}}^{\prime} \Psi_{Y_{(-1)}}=\left[a_{i j}\right]
$$

em que

$a_{11}=\sum_{t=2}^{T} \phi_{00}^{2}\left(\frac{t}{T}\right) Y_{t-1, T}^{2}$,

$a_{1 j}=a_{j 1}=\sum_{t=2}^{T} \phi_{00}\left(\frac{t}{T}\right) \psi_{j k}\left(\frac{t}{T}\right) Y_{t-1, T}^{2}, j=0,1, \cdots, j^{*}-1$ e $k=0,1, \cdots, 2^{j}-1$, $a_{i j}=a_{j i}=\sum_{t=2}^{T} \psi_{j k}\left(\frac{t}{T}\right) \psi_{j^{\prime} k^{\prime}}\left(\frac{t}{T}\right) Y_{t-1, T}^{2} \operatorname{com} j$ e $j^{\prime}=0,1, \cdots, j^{*}-1$, $k$ e $k^{\prime}=$ $0,1, \cdots, 2^{j}-1$;

$$
\Psi_{X}^{\prime} \Psi_{Y_{(-1)}}=\Psi_{Y_{(-1)}}^{\prime} \Psi_{X}=\left[b_{i j}\right]
$$

em que

$$
\begin{aligned}
& b_{11}=\sum_{t=2}^{T} \phi_{00}^{2}\left(\frac{t}{T}\right) Y_{t-1, T} X_{t, T} \\
& b_{1 j}=b_{j 1}=\sum_{t=2}^{T} \phi_{00}\left(\frac{t}{T}\right) \psi_{j k}\left(\frac{t}{T}\right) Y_{t-1, T} X_{t, T}, j=0,1, \cdots, j^{*}-1 \text { e } k=0,1, \cdots, 2^{j}-1, \\
& b_{i j}=b_{j i}=\sum_{t=2}^{T} \psi_{j k}\left(\frac{t}{T}\right) \psi_{j^{\prime} k^{\prime}}\left(\frac{t}{T}\right) Y_{t-1, T} X_{t, T} \operatorname{com} j \text { e } j^{\prime}=0,1, \cdots, j^{*}-1, k \text { e } \\
& k^{\prime}=0,1, \cdots, 2^{j}-1 ;
\end{aligned}
$$

$$
\Psi_{X}^{\prime} \Psi_{X}=\left[c_{i j}\right]
$$

em que

$$
\begin{aligned}
& c_{11}=\sum_{t=2}^{T} \phi_{00}^{2}\left(\frac{t}{T}\right) X_{t, T}^{2}, \\
& c_{1 j}=c_{j 1}=\sum_{t=2}^{T} \phi_{00}\left(\frac{t}{T}\right) \psi_{j k}\left(\frac{t}{T}\right) X_{t, T}^{2}, j=0,1, \cdots, j^{*}-1 \text { e } k=0,1, \cdots, 2^{j}-1, \\
& c_{i j}=c_{j i}=\sum_{t=2}^{T} \psi_{j k}\left(\frac{t}{T}\right) \psi_{j^{\prime} k^{\prime}}\left(\frac{t}{T}\right) X_{t, T}^{2} \operatorname{com} j \text { e } j^{\prime}=0,1, \cdots, j^{*}-1, k \text { e } k^{\prime}= \\
& 0,1, \cdots, 2^{j}-1 .
\end{aligned}
$$




\subsubsection{Propriedades Estatísticas}

Para o desenvolvimento das propriedades estatísticas são necessárias as seguintes suposições, como em Dahlhaus et al. (1999).

\section{Suposições.}

Vamos supor que cada uma das funções $\omega_{j}(u), j=0,1,2, \cdots, s$ e $\delta_{j}(u), j=$ $1,2, \cdots, r$ são funções pertencentes aos espaços de funções $\mathcal{F}_{i}$, com parâmetros $s_{i}$, $p_{i}, q_{i}$ e $m_{i}$ dado por

$$
\mathcal{F}_{i}=\left\{f=\sum_{k} \alpha_{l k}^{(i)} \phi_{l k}+\sum_{j, k} \beta_{j k}^{(i)} \psi_{j k} \mid\left\|\alpha_{l .}^{(i)}\right\|_{\infty} \leq C_{i 1},\left\|\beta_{. .}^{(i)}\right\|_{m_{i}, p_{i}, q_{i}} \leq C_{i 2}\right\}
$$

onde

$$
\left\|\beta_{. .}^{(i)}\right\|_{m_{i}, p_{i}, q_{i}}=\left(\sum_{j \geq l}\left[2^{j s_{i} p_{i}} \sum_{k \in I_{j}}\left|\beta_{j k}^{(i)}\right|^{p_{i}}\right]^{q_{i} / p_{i}}\right)^{1 / q_{i}}
$$

$s_{i}=m_{i}+1 / 2-1 / p_{i}$. Aqui, $m_{i}$ denota o grau de regularidade (suaviazação) de $\mathcal{F}_{i}$ e $p_{i}$ e $q_{i}\left(1 \leq p_{i}, q_{i} \leq \infty\right)$ especificam a norma de $\mathcal{F}_{i}$. Temos também que $C_{i 1} e C_{i 2}$ são constantes positivas. Para este espaço de funções a seguinte suposição é válida:

$$
\sup _{f_{i} \in \mathcal{F}_{i}}\left\{\sum_{j \geq j^{*}} \sum_{k}\left|\beta_{j k}^{(i)}\right|^{2}\right\}=O\left(2^{-2 j^{*} \bar{s}_{i}}\right)
$$

em que $\tilde{s}_{i}=m_{i}+1 / 2-1 / \tilde{p}_{i}, \operatorname{com} \tilde{p}_{i}=\min \left\{p_{i}, 2\right\}$.

Exemplos de tais espaços são os espaços de Besov, Sobolev, Hölder etc. Veja Vidakovic (1999), Dahlhaus et al. (1999) e Triebel (1992) para detalhes.

Demonstra-se, ver Donoho et al. (1995), que a perda na reconstrução das funções em (3.4), em decorrência da truncagem na decomposição em ondaletas no nível $j^{*}$, é de ordem $T^{-2 m_{2} /\left(2 m_{i}+1\right)}$, se escolhermos $j^{*}$ tal que $2^{j^{*}-1} \leq T^{1 / 2} \leq 2^{j^{*}}$. 


\section{Suposição 1:}

(i) $\phi$ e $\psi$ são $C^{r}[0,1]$ e têm suporte compacto;

(ii) $\int \phi(t) d t=1, \int \psi(t) t^{k}=0$ para $0 \leq k \leq r$.

No item $(i)$ temos que $\phi$ e $\psi$ são funções, definidas em $(0,1]$, com derivadas contínuas até ordem $r$, em que $r>m, m=\max \left\{m_{i}\right\}$. As ondaletas periódicas satisfazem essa suposição. Usamos ondaletas periódicas para contornar o problema das fronteiras, uma vez que estamos trabalhando com um conjunto finito de dados. A regra periódica é assumir que $X_{t+T k}=X_{t}, t=0,1, \cdots, T-1, k \in \mathbb{Z}$. Outra regra, também muito usada, é a regra de reflexão em que $X_{T+k}=X_{T-k}$, $k=1,2, \cdots, T-1$ e $X_{k}=X_{-k}, k=-T,-T+1, \cdots,-2,-1$.

\section{Suposiçāo 2:}

Existe algum $\gamma \geq 0$ tal que, $\left|\operatorname{cum}_{n}\left(\epsilon_{t}\right)\right| \leq C^{n}(n !)^{1+\gamma}, \forall n, t$. Aqui, $C$ é uma constante positiva. A distribuição normal satisfaz essa suposição. Para $\gamma=0$, essa condição é verificada para as distribuições Gama e Normal Inversa. Já para algumas distribuições com caudas mais pesadas a condição é verificada com $\gamma>0$.

\section{Suposição 3:}

Existe um $\rho>0$ tal que $1+\sum_{i=1}^{r} \delta_{i}(s) z^{i} \neq 0, \forall|z| \leq 1+\rho$ e $\forall s \in[0,1]$. Esta suposição já foi feita anteriormente, pois estamos trabalhando com funções de transferência de sistemas dinâmicos estáveis; com ela temos a garantia de uniformidade contínua das covariâncias de $\left\{X_{t, T}\right\}$ e $\left\{Y_{t, T}\right\}$, apresentada no Lema A.3.1.

Temos ainda que $C_{1} \leq \sigma^{2}(u) \leq C_{2}$, em que $u=t / T$ e no nosso caso $\sigma^{2}(u)=\sigma^{2}$. 


\section{Suposição 4:}

$\tilde{s}_{i}>1, \operatorname{com} \tilde{s}_{i}=m_{i}+1 / 2-1 / \tilde{p}_{i}$ e $\tilde{p}_{i}=\min \left\{p_{i}, 2\right\}$. É a restrição dentro do espaço de funções $\mathcal{F}_{i}$.

\section{Suposição 5:}

(i) $\sum_{(j, k) \in \mathcal{J}_{T}} P\left(\hat{\lambda}_{i j k}<\gamma_{T} \lambda_{i j k}\right)=O\left(T^{\eta}\right)$, com $\eta<1 /\left(2 m_{i}+1\right)$, para algum $\gamma_{T} \rightarrow 1$

(ii) $\sum_{(j, k) \in \mathcal{J}_{T}} P\left(\hat{\lambda}_{i j k}>C T^{-1 / 2} \sqrt{\log (T)}\right)=O\left(T^{-1}\right)$,

em que $\mathcal{J}_{T}=\left\{(j, k) \mid 2^{j} \leq T^{1 / 2}, k \in I_{j}\right\}, \sigma_{i j k}^{2}$ é a variância do coeficiente empírico $\hat{\beta}_{j k}^{(i)}$ e $\lambda_{i j k}$ é um limiar que satisfaz

$$
\sigma_{i j k} \sqrt{2 \log \left(2^{j^{*}}-1\right)} \leq \lambda_{i j k}=O\left(T^{-1 / 2} \sqrt{\log (T)}\right)
$$

Usamos $\lambda_{i j k}=\sigma_{i j k} \sqrt{2 \log \left(2^{j^{*}}-1\right)}$ se adotamos um limiar diferente para cada nível, porém, se a escolha for por um limiar único teremos $\lambda_{T}^{(i)}=\sigma_{T}^{(i)} \sqrt{2 \log \left(2^{j^{*}}-1\right)}$, em que $\sigma_{T}^{(i)}=\max _{(j, k) \in \mathcal{J}_{T}}\left\{\sigma_{i j k}\right\}, i=\delta_{1}, \cdots, \delta_{\tau}, \omega_{0}, \cdots, \omega_{s}$.

\section{Suposição 6:}

$$
\mathbb{E}\left\|\left(\Upsilon^{\prime} \Upsilon\right)^{-1}\right\|_{L_{2}}^{2+\delta}=O\left(T^{-2-\delta}\right), \text { para algum } \delta>0
$$

com $\Upsilon$ dada em (3.18) a seguir.

\section{Propriedades Estatísticas}

Consideremos o modelo (3.1) e suponhamos que as funções $\delta_{1}\left(\frac{t}{T}\right)$ e $\omega_{0}\left(\frac{t}{T}\right)$ sejam de quadrado integráveis, isto é, pertencem a $L^{2}((0,1])$. As propriedades 
estatísticas aqui apresentadas seguem o procedimento apresentado por Dahlhaus et al. (1999). Apesar da necessidade, para esse procedimento de estimação, de usarmos uma base de multirresolução $V_{0} \oplus W_{1} \oplus W_{2} \oplus \cdots \oplus W_{j^{*-1}}$ em que temos os coeficientes em diferentes níveis de resolução, é mais fácil analisar as propriedades estatísticas dos coeficientes em um único nível $V_{j^{*}}$. Os coeficientes da base de multirresolução são obtidos através de combinações lineares dos coeficientes encontrados na base de $V_{j^{*}}$, que é a base gerada por $\left\{\phi_{j^{*}, 1}, \phi_{j^{*}, 2}, \cdots, \phi_{j^{*}, 2^{j^{*}}}\right\}$. Nesta base, podemos escrever (3.1) como

$$
Y_{t, T}=\sum_{i=1}^{2^{j^{*}}} \zeta_{j^{*}, i}^{\left(\delta_{1}\right)} \phi_{j^{*}, i}\left(\frac{t}{T}\right) Y_{t-1, T}(t)+\sum_{i=1}^{2^{j^{*}}} \zeta_{j^{*}, i}^{\left(\omega_{0}\right)} \phi_{j^{*}, i}\left(\frac{t}{T}\right) X_{t, T}+\gamma_{t, T},
$$

em que

$$
\gamma_{t, T}=\sum_{j \geq j^{*}} \sum_{k \in I_{j}} \beta_{j k}^{\left(\delta_{1}\right)} \psi_{j k}\left(\frac{t}{T}\right) Y_{t-1, T}+\sum_{j \geq j^{*}} \sum_{k \in I_{j}} \beta_{j k}^{\left(\omega_{0}\right)} \psi_{j k}\left(\frac{t}{T}\right) X_{t, T}+e_{t, T}
$$

Escrevendo a equação (3.12) na forma matricial temos:

$$
\mathbf{Y}=\left[\begin{array}{lll}
\varphi_{Y_{(-1)}} & \vdots & \varphi_{X}
\end{array}\right]\left[\begin{array}{c}
\zeta^{\left(\delta_{1}\right)} \\
\ldots \\
\boldsymbol{\zeta}^{\left(\omega_{0}\right)}
\end{array}\right]+\gamma
$$

com

$$
\boldsymbol{\varphi}_{Y_{(-1)}}=\left[\begin{array}{cccc}
\phi_{j^{*}, 1}\left(\frac{2}{T}\right) Y_{1, T} & \phi_{j^{*}, 2}\left(\frac{2}{T}\right) Y_{1, T} & \cdots & \phi_{j^{*}, 2^{*}}\left(\frac{2}{T}\right) Y_{1, T} \\
\phi_{j^{*}, 1}\left(\frac{3}{T}\right) Y_{2, T} & \phi_{j^{*}, 2}\left(\frac{3}{T}\right) Y_{2, T} & \cdots & \phi_{j^{*}, 2^{*}}\left(\frac{3}{T}\right) Y_{2, T} \\
\vdots & \vdots & \ddots & \vdots \\
\phi_{j^{*}, 1}\left(\frac{T}{T}\right) Y_{T-1, T} & \phi_{j^{*}, 2}\left(\frac{T}{T}\right) Y_{T-1, T} & \cdots & \phi_{j^{*}, 2^{j^{*}}}\left(\frac{T}{T}\right) Y_{T-1, T}
\end{array}\right]
$$

e

$$
\varphi_{X}=\left[\begin{array}{cccc}
\phi_{j^{*}, 1}\left(\frac{2}{T}\right) X_{2, T} & \phi_{j^{*}, 2}\left(\frac{2}{T}\right) X_{2, T} & \cdots & \phi_{j^{*}, 2^{*}}\left(\frac{2}{T}\right) X_{2, T} \\
\phi_{j^{*}, 1}\left(\frac{3}{T}\right) X_{3, T} & \phi_{j^{*}, 2}\left(\frac{3}{T}\right) X_{3, T} & \cdots & \phi_{j^{*}, 2^{*}}\left(\frac{3}{T}\right) X_{3, T} \\
\vdots & \vdots & \ddots & \vdots \\
\phi_{j^{*}, 1}\left(\frac{T}{T}\right) X_{T, T} & \phi_{j^{*}, 2}\left(\frac{T}{T}\right) X_{T . T} & \cdots & \phi_{j^{*}, 2^{*}}\left(\frac{T}{T}\right) X_{T, T}
\end{array}\right] .
$$


Segue-se analogamente às equações (3.10), que

$$
\left[\begin{array}{l}
\hat{\zeta}^{\left(\delta_{1}\right)} \\
\hat{\zeta}^{\left(\omega_{0}\right)}
\end{array}\right]=\left[\begin{array}{ll}
\varphi_{Y_{(-1)}}^{\prime} \varphi_{Y_{(-1)}} & \varphi_{Y_{(-1)}}^{\prime} \varphi_{X} \\
\varphi_{X}^{\prime} \varphi_{Y_{(-1)}} & \varphi_{X}^{\prime} \varphi_{X}
\end{array}\right]^{-1}\left[\begin{array}{l}
\varphi_{Y_{(-1)}}^{\prime} Y \\
\varphi_{X}^{\prime} Y
\end{array}\right]
$$

A relação entre $\left(\begin{array}{l}\boldsymbol{\beta}^{\left(\delta_{1}\right)} \\ \boldsymbol{\beta}^{\left(\omega_{0}\right)}\end{array}\right)$ e $\left(\begin{array}{l}\boldsymbol{\zeta}^{\left(\delta_{1}\right)} \\ \boldsymbol{\zeta}^{\left(\omega_{0}\right)}\end{array}\right)$, isto é, os coeficientes apresentados em $(3.10)$, base de multirresolução, e os coeficientes apresentados em (3.17) é

$$
\left(\begin{array}{l}
\beta^{\left(\delta_{1}\right)} \\
\beta^{\left(\omega_{0}\right)}
\end{array}\right)=\Gamma\left(\begin{array}{l}
\zeta^{\left(\delta_{1}\right)} \\
\zeta^{\left(\omega_{0}\right)}
\end{array}\right)
$$

onde $\Gamma$ é uma matriz, de dimensão $2^{j^{*}+1} \times 2^{j^{*}+1}$, bloco diagonal. O $i$-ésimo bloco de $\Gamma$ é $\Gamma_{i}=\mathbf{W V}^{\prime}$ em que $\mathbf{W}$ é a base ortonormal gerada por $\left\{\phi_{00}, \psi_{00}, \psi_{10}, \cdots\right.$, $\left.\psi_{j^{*}-1,2^{j^{*}-1}-1}\right\}$ e V é a base ortonormal gerada por $\left\{\phi_{j^{*}, 1}, \phi_{j^{*}, 2}, \cdots, \phi_{j^{*}, 2^{j^{*}}}\right\}$.

A matriz $\Gamma$ faz a transformação $\left(\hat{\alpha}_{00}^{(i)}, \hat{\beta}_{00}^{(i)}, \hat{\beta}_{10}^{(i)}, \hat{\beta}_{11}^{(i)}, \cdots, \hat{\beta}_{j^{*}-1,0}^{(i)}, \cdots, \hat{\beta}_{j^{*}-1, \Delta}^{(i)}\right)^{\prime}$ $=\Gamma\left(\hat{\zeta}_{j^{*}, 1}^{(i)}, \cdots, \hat{\zeta}_{j^{*}, 2^{j^{*}}}^{(i)}\right)^{\prime}$. Nesse caso, $\boldsymbol{\Gamma}=\operatorname{diag}\left[\Gamma_{\delta_{1}}, \Gamma_{\omega_{0}}\right]$ e $\Gamma_{\delta_{1}}=\Gamma_{\omega_{0}}=\Gamma_{i}$ em que,

$$
\Gamma_{i}=\left[\begin{array}{ccc}
\sum_{t=0}^{T-1} \phi_{00}(t) \phi_{j^{*}, 1}(t) & \cdots & \sum_{t=0}^{T-1} \phi_{00}(t) \phi_{j^{*}, 2^{*}}(t) \\
\sum_{t=0}^{T-1} \psi_{00}(t) \phi_{j^{*}, 1}(t) & \cdots & \sum_{t=0}^{T-1} \psi_{00}(t) \phi_{j^{*}, 2^{j^{*}}}(t) \\
\sum_{t=0}^{T-1} \psi_{10}(t) \phi_{j^{*}, 1}(t) & \cdots & \sum_{t=0}^{T-1} \psi_{10}(t) \phi_{j^{*}, 2^{j^{*}}}(t) \\
\sum_{t=0}^{T-1} \psi_{11}(t) \phi_{j^{*}, 1}(t) & \cdots & \sum_{t=0}^{T-1} \psi_{11}(t) \phi_{j^{*}, 2^{j^{*}}}(t) \\
\vdots & \ddots & \vdots \\
\sum_{t=0}^{T-1} \psi_{j^{*}-1,0}(t) \phi_{j^{*}, 1}(t) & \cdots & \sum_{t=0}^{T-1} \psi_{j^{*}-1,0}(t) \phi_{j^{*}, 2^{*}}(t) \\
\vdots & \ddots & \vdots \\
\sum_{t=0}^{T-1} \psi_{j^{*}-1, \Delta}(t) \phi_{j^{*}, 1}(t) & \cdots & \sum_{t=0}^{T-1} \psi_{j^{*}-1, \Delta}(t) \phi_{j^{*}, 2^{*}}(t)
\end{array}\right]
$$

Assim cada estimativa do coeficiente $\boldsymbol{\beta}^{(i)}, i=\delta_{1}, \omega_{0}$ de (3.10) pode ser escrita como

$$
\begin{aligned}
& \hat{\boldsymbol{\beta}}^{\left(\delta_{1}\right)}=\Gamma_{\delta_{1}} \hat{\zeta}^{\left(\delta_{1}\right)}, \\
& \hat{\boldsymbol{\beta}}^{\left(\omega_{0}\right)}=\Gamma_{\omega_{0}} \hat{\zeta}^{\left(\omega_{0}\right)},
\end{aligned}
$$


onde $\left\|\Gamma_{\delta_{1}, j, k}\right\|_{L_{2}}=\left\|\Gamma_{\omega_{0}, j, k}\right\|_{L_{2}}=1$

A equação (3.14) pode ser escrita na forma

$$
\mathbf{Y}=\Upsilon \zeta+\gamma
$$

$\operatorname{com} \boldsymbol{\Upsilon}=\left[\boldsymbol{\varphi}_{Y_{(-1)}} \boldsymbol{\varphi}_{X}\right], \boldsymbol{\zeta}=\left(\begin{array}{c}\boldsymbol{\zeta}^{\left(\delta_{1}\right)} \\ \boldsymbol{\zeta}^{\left(\omega_{0}\right)}\end{array}\right)$ e $\boldsymbol{\gamma}$ vetor cujas componentes são dadas por (3.13).

O estimador de $\zeta$ é $\hat{\zeta}$, dado por

$$
\hat{\zeta}=\left(\Upsilon^{\prime} \Upsilon\right)^{-1} \Upsilon^{\prime} \mathbf{Y}
$$

De (3.18) e (3.19) temos

$$
\hat{\zeta}=\left(\Upsilon^{\prime} \Upsilon\right)^{-1} \Upsilon^{\prime}(\Upsilon \zeta+\gamma)
$$

Se escrevermos $\gamma=\mathrm{e}+\mathrm{S}$, obtemos

$$
\hat{\zeta}=\left(\Upsilon^{\prime} \Upsilon\right)^{-1} \Upsilon^{\prime}(\Upsilon \zeta+e+S)
$$

onde a matriz $\mathbf{S}$, de ordem $(T-1) \times 1$, é dada por

$$
\mathbf{S}=\left[\begin{array}{c}
\sum_{j \geq j^{*}} \sum_{k \in \mathcal{I}_{j}} \beta_{j k}^{\left(\delta_{1}\right)} \psi_{j k}\left(\frac{2}{T}\right) Y_{1, T}+\sum_{j \geq j^{*}} \sum_{k \in \mathcal{I}_{j}} \beta_{j k}^{\left(\omega_{0}\right)} \psi_{j k}\left(\frac{2}{T}\right) X_{2, T} \\
\sum_{j \geq j^{*}} \sum_{k \in \mathcal{I}_{j}} \beta_{j k}^{\left(\delta_{1}\right)} \psi_{j k}\left(\frac{3}{T}\right) Y_{2, T}+\sum_{j \geq j^{*}} \sum_{k \in \mathcal{I}_{j}} \beta_{j k}^{\left(\omega_{0}\right)} \psi_{j k}\left(\frac{3}{T}\right) X_{3, T} \\
\vdots \\
\sum_{j \geq j^{*}} \sum_{k \in \mathcal{I}_{j}} \beta_{j k}^{\left(\delta_{1}\right)} \psi_{j k}\left(\frac{T}{T}\right) Y_{T-1, T}+\sum_{j \geq j^{*}} \sum_{k \in \mathcal{I}_{j}} \beta_{j k}^{\left(\omega_{0}\right)} \psi_{j k}\left(\frac{T}{T}\right) X_{T, T}
\end{array}\right]
$$

Note que

$$
\varphi_{Y_{(-1)}}^{\prime} \mathbf{e}=\left[\begin{array}{c}
\sum_{t=2}^{T} \phi_{j^{*}, 1}\left(\frac{t}{T}\right) Y_{t-1, T} e_{t, T} \\
\sum_{t=2}^{T} \phi_{j^{*}, 2}\left(\frac{t}{T}\right) Y_{t-1, T} e_{t, T} \\
\vdots \\
\sum_{t=2}^{T} \phi_{j^{*}, 2^{*}}\left(\frac{t}{T}\right) Y_{t-1, T} e_{t, T}
\end{array}\right]
$$




$$
\varphi_{X}^{\prime} \mathrm{e}=\left[\begin{array}{c}
\sum_{t=2}^{T} \phi_{j^{*}, 1}\left(\frac{t}{T}\right) X_{t, T} e_{t, T} \\
\sum_{t=2}^{T} \phi_{j^{*}, 2}\left(\frac{t}{T}\right) X_{t, T} e_{t, T} \\
\vdots \\
\sum_{t=2}^{T} \phi_{j^{*}, 2^{*}}\left(\frac{t}{T}\right) X_{t, T} e_{t, T}
\end{array}\right]
$$

Proposição 3.1: Sob as suposições (1)-(6) temos que $\|\hat{\zeta}-\zeta\|_{\infty}^{2}=O_{p}\left(2^{j^{*}} T^{-1} \log (T)\right)$.

Prova. Temos

$$
\begin{aligned}
\hat{\boldsymbol{\zeta}} & =\left(\Upsilon^{\prime} \Upsilon\right)^{-1} \Upsilon^{\prime}(\Upsilon \boldsymbol{\zeta}+\boldsymbol{\gamma}) \\
& =\left(\Upsilon^{\prime} \Upsilon\right)^{-1} \Upsilon^{\prime} \Upsilon \boldsymbol{\zeta}+\left(\Upsilon^{\prime} \Upsilon\right)^{-1} \Upsilon^{\prime}(\mathbf{e}+\mathbf{S})
\end{aligned}
$$

que implica

$$
\begin{aligned}
\hat{\boldsymbol{\zeta}}-\boldsymbol{\zeta} & =\left(\Upsilon^{\prime} \Upsilon\right)^{-1}\left(\Upsilon^{\prime} \mathbf{e}+\Upsilon^{\prime} \mathbf{S}\right) \\
& =\left(\Upsilon^{\prime} \Upsilon\right)^{-1} \Upsilon^{\prime} \mathbf{e}+\left(\boldsymbol{\Upsilon}^{\prime} \Upsilon\right)^{-1} \Upsilon^{\prime} \mathbf{S} \\
& =\left[\left(\mathbb{E} \Upsilon^{\prime} \Upsilon\right)^{-1}-\left(E \Upsilon^{\prime} \Upsilon\right)^{-1}+\left(\Upsilon^{\prime} \Upsilon\right)^{-1}\right] \Upsilon^{\prime} e+\left(\Upsilon^{\prime} \Upsilon\right)^{-1} \Upsilon^{\prime} S \\
& =\left(E \Upsilon^{\prime} \Upsilon\right)^{-1} \Upsilon^{\prime} e+\left[\left(\Upsilon^{\prime} \Upsilon\right)^{-1}-\left(E \Upsilon^{\prime} \Upsilon\right)^{-1}\right] \Upsilon^{\prime} e+\left(\Upsilon^{\prime} \Upsilon\right)^{-1} \Upsilon^{\prime} S
\end{aligned}
$$

Escrevamos

$$
\hat{\zeta}-\zeta=T_{1}+T_{2}+T_{3}
$$

em que $T_{1}=\left(\mathbb{E} \boldsymbol{\Upsilon}^{\prime} \boldsymbol{\Upsilon}\right)^{-1} \boldsymbol{\Upsilon}^{\prime} \mathbf{e}, T_{2}=\left[\left(\boldsymbol{\Upsilon}^{\prime} \boldsymbol{\Upsilon}\right)^{-1}-\left(\mathbb{E} \boldsymbol{\Upsilon}^{\prime} \boldsymbol{\Upsilon}\right)^{-1}\right] \boldsymbol{\Upsilon}^{\prime} \mathrm{e}$ e $T_{3}=\left(\Upsilon^{\prime} \Upsilon\right)^{-1} \Upsilon^{\prime} \mathbf{S}$

Como $e_{t, T}=\epsilon_{t}+\delta_{1}(t) \epsilon_{t-1}$, temos que,

$$
\Upsilon^{\prime} \mathrm{e}=\mathbf{\Upsilon}^{\prime}\left[\epsilon+\delta_{1} \epsilon_{(-1)}\right]
$$

em que $\epsilon=\left(\epsilon_{2}, \epsilon_{3}, \cdots, \epsilon_{T}\right)^{\prime}$ e $\delta_{1} \epsilon_{(-1)}=\left(\delta_{1}(2) \epsilon_{1}, \delta_{1}(3) \epsilon_{2}, \cdots, \delta_{1}(T) \epsilon_{T-1}\right)^{\prime}$. 
Desse modo,

$$
\begin{aligned}
T_{1} & =\left(E \Upsilon^{\prime} \Upsilon\right)^{-1} \Upsilon^{\prime} \mathbf{e} \\
& =\left(E \Upsilon^{\prime} \Upsilon\right)^{-1} \Upsilon^{\prime} \epsilon+\left(E \Upsilon^{\prime} \Upsilon\right)^{-1} \Upsilon^{\prime} \delta_{1} \epsilon_{(-1)} \\
\left\|T_{1}\right\|_{\infty} & \leq\left\|\left(E \Upsilon^{\prime} \Upsilon\right)^{-1}\right\|_{\infty}\left\|\Upsilon^{\prime} \epsilon\right\|_{\infty}+\left\|\left(E \Upsilon^{\prime} \Upsilon\right)^{-1}\right\|_{\infty}\left\|\Upsilon^{\prime} \delta_{1} \epsilon_{(-1)}\right\|_{\infty}
\end{aligned}
$$

Usando o Lema $A .3 .3(i)$ temos que $\left\|\left(\boldsymbol{E} \Upsilon^{\prime} \Upsilon\right)^{-1}\right\|_{\infty}=O\left(T^{-1}\right)$. O Lema A.3.3(iv) garante que $\left\|\Upsilon^{\prime} \epsilon\right\|_{2}^{2}=O_{p}\left(2^{j^{*}} T \log (T)\right)$, de onde $\left\|\Upsilon^{\prime} \boldsymbol{\epsilon}\right\|_{2}=$ $O_{p}\left(2^{j^{*} / 2} T^{1 / 2} \sqrt{\log (T)}\right)$. Como $\boldsymbol{\Upsilon}^{\prime} \epsilon$ é um vetor coluna, temos que $\left\|\boldsymbol{\Upsilon}^{\prime} \boldsymbol{\epsilon}\right\|_{\infty} \leq$ $\left\|\boldsymbol{\Upsilon}^{\prime} \boldsymbol{\epsilon}\right\|_{2}$ e então

$$
\left\|T_{1}\right\|_{\infty}=O_{p}\left(2^{j^{*} / 2} T^{-1 / 2} \sqrt{\log (T)}\right) .
$$

Além disso, temos que $\boldsymbol{E}\left(\Gamma_{i, j, k}^{\prime} T_{1}\right)=\Gamma_{i, j, k}^{\prime} \boldsymbol{E}\left(\left(\boldsymbol{E} \boldsymbol{\Upsilon}^{\prime} \boldsymbol{\Upsilon}\right)^{-1}\left(\boldsymbol{\Upsilon}^{\prime} \mathbf{e}\right)\right)=\Gamma_{i, j, k}^{\prime}\left(\boldsymbol{E} \boldsymbol{\Upsilon}^{\prime} \boldsymbol{\Upsilon}\right)^{-1}$ $\boldsymbol{E}\left(\boldsymbol{\Upsilon}^{\prime} \mathbf{e}\right)$. Como $\boldsymbol{E}\left(\boldsymbol{\Upsilon}^{\prime} \mathbf{e}\right)=0$ temos que $\Gamma_{i, j, k} \boldsymbol{E}\left(T_{\mathbf{1}}\right)=0$, e

$$
\begin{aligned}
& E\left(\Gamma_{i j k}^{\prime} T_{1}\right)^{2}=E\left(\Gamma_{i j k}^{\prime}\left[\left(\mathbb{E} \Upsilon^{\prime} \Upsilon\right)^{-1} \Upsilon^{\prime} \epsilon+\left(\mathbb{E} \Upsilon^{\prime} \Upsilon\right)^{-1} \Upsilon^{\prime} \delta_{1} \epsilon_{(-1)}\right]\right)^{2} \\
& =E\left(\boldsymbol{\Gamma}_{i j k}^{\prime}\left(\mathbb{E} \boldsymbol{\Upsilon}^{\prime} \Upsilon\right)^{-1} \boldsymbol{\Upsilon}^{\prime} \epsilon+\Gamma_{i j k}^{\prime}\left(\mathbb{E} \Upsilon^{\prime} \Upsilon\right)^{-1} \boldsymbol{\Upsilon}^{\prime} \delta_{1} \epsilon_{(-1)}\right)^{2}
\end{aligned}
$$

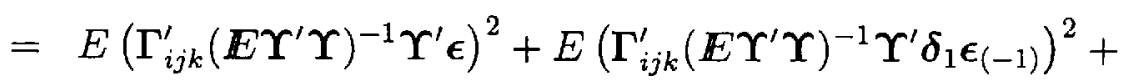

$$
\begin{aligned}
& +2 E\left(\boldsymbol{\Gamma}_{i j k}^{\prime}\left(E \Upsilon^{\prime} \Upsilon\right)^{-1} \Upsilon^{\prime} \epsilon \delta_{1} \epsilon_{(-1)}^{\prime} \boldsymbol{\Upsilon}^{\prime}\left(E \Upsilon^{\prime} \Upsilon\right)^{-1} \Gamma_{i j k}\right) \\
& =E\left(\boldsymbol{\Gamma}_{i j k}^{\prime}\left(\mathbb{E} \boldsymbol{\Upsilon}^{\prime} \boldsymbol{\Upsilon}\right)^{-1} \boldsymbol{\Upsilon}^{\prime} \boldsymbol{\epsilon} \epsilon^{\prime} \Upsilon\left(\boldsymbol{E} \boldsymbol{\Upsilon}^{\prime} \boldsymbol{\Upsilon}\right)^{-1} \boldsymbol{\Gamma}_{i j k}\right)+ \\
& +E\left(\Gamma_{i j k}^{\prime}\left(E \Upsilon^{\prime} \Upsilon\right)^{-1} \Upsilon^{\prime} \delta_{1} \epsilon_{(-1)} \delta_{1} \epsilon_{(-1)}^{\prime} \Upsilon\left(\mathbb{E} \Upsilon^{\prime} \Upsilon\right)^{-1} \Gamma_{i j k}\right)+ \\
& +2 E\left(\Gamma_{i j k}^{\prime}\left(\mathbb{E} \Upsilon^{\prime} \Upsilon\right)^{-1} \Upsilon^{\prime} \epsilon \delta_{1} \epsilon_{(-1)}^{\prime} \boldsymbol{\Upsilon}^{\prime}\left(E \Upsilon^{\prime} \Upsilon\right)^{-1} \Gamma_{i j k}\right) \\
& \leq\left\|\Gamma_{i j k}\right\|_{2}^{2}\left\|\left(\boldsymbol{E} \mathbf{\Upsilon}^{\prime} \boldsymbol{\Upsilon}\right)^{-1}\right\|_{2}^{2}\left\|\operatorname{cov}\left(\boldsymbol{\Upsilon}^{\prime} \boldsymbol{\epsilon}\right)\right\|_{2}+ \\
& +\left\|\Gamma_{i j k}\right\|_{2}^{2}\left\|\left(\mathbb{E} \Upsilon^{\prime} \boldsymbol{\Upsilon}\right)^{-1}\right\|_{2}^{2}\left\|\operatorname{cov}\left(\boldsymbol{\Upsilon}^{\prime} \boldsymbol{\delta}_{1} \boldsymbol{\epsilon}_{(-1)}\right)\right\|_{2}+ \\
& +\left\|\Gamma_{i j k}\right\|_{2}^{2}\left\|\left(\mathbb{E} \Upsilon^{\prime} \Upsilon\right)^{-1}\right\|_{2}^{2}\left\|\Upsilon^{\prime} \mathbb{E}\left(\epsilon \delta_{1} \epsilon_{(-1)}^{\prime}\right) \Upsilon\right\|_{2},
\end{aligned}
$$

e como $\boldsymbol{\epsilon}$ é ruído branco, temos $\boldsymbol{E}\left(\boldsymbol{\epsilon}^{\prime} \boldsymbol{\delta}_{1} \boldsymbol{\epsilon}_{(-1)}\right)$ é zero, e usando os Lemas $A .3 .3(i) \mathrm{e}$ (ii) temos que

$$
E\left(\Gamma_{i j k}^{\prime} T_{1}\right)^{2}=O\left(T^{-1}\right)
$$


O segundo termo, $T_{2}$, é:

$$
\begin{aligned}
T_{2} & =\left[\left(\boldsymbol{\Upsilon}^{\prime} \boldsymbol{\Upsilon}\right)^{-1}-\left(\boldsymbol{E} \boldsymbol{\Upsilon}^{\prime} \boldsymbol{\Upsilon}\right)^{-1}\right] \boldsymbol{\Upsilon}^{\prime} \mathbf{e} \\
& =\left[\left(\boldsymbol{\Upsilon}^{\prime} \Upsilon\right)^{-1}-\left(\boldsymbol{E} \boldsymbol{\Upsilon}^{\prime} \boldsymbol{\Upsilon}\right)^{-1}\right] \boldsymbol{\Upsilon}^{\prime}\left(\epsilon-\delta_{1} \epsilon_{(-1)}\right)
\end{aligned}
$$

Expandindo $\left(\boldsymbol{\Upsilon}^{\prime} \Upsilon\right)^{-1}$ em série de Taylor no ponto $\left(\boldsymbol{E} \boldsymbol{\Upsilon}^{\prime} \boldsymbol{\Upsilon}\right)^{-1}$ temos

$$
\begin{aligned}
\left(\boldsymbol{\Upsilon}^{\prime} \Upsilon\right)^{-1} & =\left(E \Upsilon^{\prime} \Upsilon\right)^{-1}+\left(E \Upsilon^{\prime} \Upsilon\right)^{-1}\left(E \Upsilon^{\prime} \Upsilon-\Upsilon^{\prime} \Upsilon\right)\left(E \Upsilon^{\prime} \Upsilon\right)^{-1}+ \\
& +O_{p}\left(\left(E \Upsilon^{\prime} \Upsilon\right)^{-3}\left(E \Upsilon^{\prime} \Upsilon-\Upsilon^{\prime} \Upsilon\right)^{2}\right)
\end{aligned}
$$

o que implica em

$$
\begin{aligned}
\left(\boldsymbol{\Upsilon}^{\prime} \Upsilon\right)^{-1}-\left(\boldsymbol{E} \boldsymbol{\Upsilon}^{\prime} \boldsymbol{\Upsilon}\right)^{-1} & =\left(\boldsymbol{E} \boldsymbol{\Upsilon}^{\prime} \boldsymbol{\Upsilon}\right)^{-1}\left(\boldsymbol{E} \boldsymbol{\Upsilon}^{\prime} \boldsymbol{\Upsilon}-\boldsymbol{\Upsilon}^{\prime} \Upsilon\right)\left(\boldsymbol{E} \Upsilon^{\prime} \Upsilon\right)^{-1}+ \\
& +O_{p}\left(\left(\boldsymbol{E} \boldsymbol{\Upsilon}^{\prime} \Upsilon\right)^{-3}\left(\boldsymbol{E} \Upsilon^{\prime} \Upsilon-\Upsilon^{\prime} \Upsilon\right)^{2}\right)
\end{aligned}
$$

Assim,

$$
\begin{aligned}
T_{2}= & {\left[\left(\mathbb{E} \Upsilon^{\prime} \Upsilon\right)^{-1}\left(\mathbb{E} \Upsilon^{\prime} \Upsilon-\Upsilon^{\prime} \Upsilon\right)\left(E \Upsilon^{\prime} \Upsilon\right)^{-1}+\right.} \\
& \left.+O_{p}\left(\left(E \Upsilon^{\prime} \Upsilon\right)^{-3}\left(E \Upsilon^{\prime} \Upsilon-\Upsilon^{\prime} \Upsilon\right)^{2}\right)\right] \Upsilon^{\prime}\left(\epsilon-\delta_{1} \epsilon_{(-1)}\right) \\
= & T_{21}+T_{22}+T_{23}+T_{24}
\end{aligned}
$$

em que,

$$
\begin{aligned}
& T_{21}=\left[\left(\boldsymbol{E} \boldsymbol{\Upsilon}^{\prime} \boldsymbol{\Upsilon}\right)^{-1}\left(\boldsymbol{E} \boldsymbol{\Upsilon}^{\prime} \boldsymbol{\Upsilon}-\boldsymbol{\Upsilon}^{\prime} \boldsymbol{\Upsilon}\right)\left(\boldsymbol{E} \boldsymbol{\Upsilon}^{\prime} \boldsymbol{\Upsilon}\right)^{-1}\right] \mathbf{\Upsilon}^{\prime} \boldsymbol{\epsilon}, \\
& T_{22}=O_{p}\left(\left(\boldsymbol{E} \Upsilon^{\prime} \Upsilon\right)^{-3}\left(\mathbb{E} \Upsilon^{\prime} \Upsilon-\Upsilon^{\prime} \Upsilon\right)^{2}\right) \Upsilon^{\prime} \boldsymbol{\epsilon},
\end{aligned}
$$

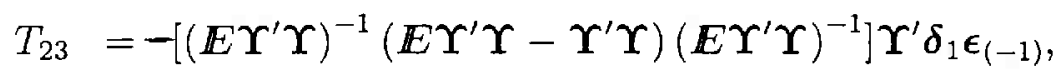

$$
\begin{aligned}
& T_{24}=-O_{p}\left(\left(\mathbb{E} \Upsilon^{\prime} \Upsilon\right)^{-3}\left(\mathbb{E} \Upsilon^{\prime} \Upsilon-\Upsilon^{\prime} \Upsilon\right)^{2}\right) \Upsilon^{\prime} \delta_{1} \epsilon_{(-1)} \text {. }
\end{aligned}
$$

Usando o Lema $A .3 .3(i)$, a equação (A.7) e o Lema $A .3 .3(i v)$ temos,

$$
\begin{aligned}
\left\|T_{21}\right\|_{\infty} & \leq\left\|\left(E \Upsilon^{\prime} \Upsilon\right)^{-1}\right\|_{\infty}^{2}\left\|E \Upsilon^{\prime} \Upsilon-\Upsilon^{\prime} \Upsilon\right\|_{\infty}\left\|\Upsilon^{\prime} \epsilon\right\|_{\infty} \\
& =O_{p}\left(T^{-1} 2^{j^{*}} \log (T)\right)
\end{aligned}
$$


Já para $\left\|T_{22}\right\|_{\infty}$ o resultado é:

$$
\left\|T_{22}\right\|_{\infty} \leq\left(\left\|\left(E \Upsilon^{\prime} \Upsilon\right)^{-1}\right\|_{2}^{3}\left\|E \Upsilon^{\prime} \Upsilon-\Upsilon^{\prime} \Upsilon\right\|_{2}^{2}\left\|\boldsymbol{\Upsilon}^{\prime} \epsilon\right\|_{2}\right) .
$$

Utilizando o Lema $A .3 .3(i)$, a equação (A.7) e o Lema $A .3 .3(i v)$, temos que

$$
\left\|T_{22}\right\|_{\infty} \leq\left\|T_{22}\right\|_{2}=O_{p}\left(T^{-3 / 2} 2^{j^{*}} \sqrt{(\log (T))^{3}}\right) .
$$

Uma vez que $\left|\delta_{1}(t)\right|<1$ para $t=1,2, \cdots, T$, os resultados para $T_{23}$ e $T_{24}$ são de mesma ordem que $T_{21}$ e $T_{22}$, respectivamente. Assim,

$$
\left\|T_{2}\right\|_{\infty}=O_{p}\left(T^{-1} 2^{j^{*}} \log (T)\right) .
$$

Quanto ao terceiro termo, $T_{3}=\left(\Upsilon^{\prime} \Upsilon\right)^{-1} \Upsilon^{\prime} S$, temos que,

$$
\begin{aligned}
\left\|\left(\boldsymbol{\Upsilon}^{\prime} \boldsymbol{\Upsilon}\right)^{-1}\right\|_{2} & \leq\left\|\left(\boldsymbol{E}^{\prime} \boldsymbol{\Upsilon}\right)^{-1}\right\|_{2}+\left\|\left(\boldsymbol{\Upsilon}^{\prime} \boldsymbol{\Upsilon}\right)^{-1}-\left(\boldsymbol{E} \boldsymbol{\Upsilon}^{\prime} \boldsymbol{\Upsilon}\right)^{-1}\right\|_{2} \\
& =O\left(T^{-1}\right)+O_{p}\left(2^{j^{*} / 2} T^{-3 / 2} \sqrt{\log (T)}\right)
\end{aligned}
$$

Esse resultado foi obtido usando os Lemas $A .3 .3(i)$ e $A .3 .3(i i)$.

Do Lema $A .3 .3(v)$ temos que,

$$
\left\|\Upsilon^{\prime} \mathbf{S}\right\|_{2}=O_{p}\left(T\left(2^{-j^{*} \min \left\{\bar{s}_{i}\right\}}+T^{-1 / 2} 2^{-j^{*} \min \left\{m_{i}-\frac{1}{2}-\frac{1}{2 p_{i}}\right\}}\right) \sqrt{\log (T)}\right) .
$$

Então

$$
\left\|T_{3}\right\|_{2}=O_{p}\left(\left(2^{-j^{*} \min \left\{\bar{s}_{i}\right\}}+T^{-1 / 2} 2^{-j^{*} \min \left\{m_{i}-\frac{1}{2}-\frac{1}{2 p_{i}}\right\}}\right) \sqrt{\log (T)}\right) .
$$

Dessa forma, podemos majorar (3.25) por $\left\|T_{3}\right\|_{2}=O_{p}\left(T^{-1 / 2-\tau}\right)$ para algum $\tau>0$. Como $T_{3}$ é um vetor coluna, temos que $\left\|T_{3}\right\|_{\infty} \leq\left\|T_{3}\right\|_{2}$.

Assim, de (3.21), (3.24) e (3.25) temos,

$$
\begin{aligned}
\|\boldsymbol{\zeta}-\hat{\boldsymbol{\zeta}}\|_{\infty} & =\left\|T_{1}+T_{2}+T_{3}\right\|_{\infty} \\
& \leq\left\|T_{1}\right\|_{\infty}+\left\|T_{2}\right\|_{\infty}+\left\|T_{3}\right\|_{\infty} \\
& =O_{p}\left(2^{j^{*} / 2} T^{-1 / 2} \sqrt{\log (T)}\right),
\end{aligned}
$$


o que completa a prova.

Encontramos $\beta_{j k}^{(i)}$ através da transformação linear $\beta_{j k}^{(i)}=\Gamma_{i, j, k}^{\prime} \boldsymbol{\zeta}$, assim $\hat{\beta}_{j k}^{(i)}=$ $\beta_{j k}^{(i)}+\Gamma_{i, j, k}^{\prime} T_{1}+\Gamma_{i, j, k}^{\prime} T_{2}+\Gamma_{i, j, k}^{\prime} T_{3}$ e $\beta_{j k}^{(i)}-\hat{\beta}_{j k}^{(i)}=\Gamma_{i, j, k}^{\prime}(\zeta-\hat{\zeta})$. Temos então, que $\left\|\beta_{j k}^{(i)}-\hat{\beta}_{j k}^{(i)}\right\|_{\infty} \leq\left\|\Gamma_{i, j, k}^{\prime}\right\|_{\infty}\|\boldsymbol{\zeta}-\hat{\boldsymbol{\zeta}}\|_{\infty}=\|\boldsymbol{\zeta}-\hat{\boldsymbol{\zeta}}\|_{\infty}$ uma vez que $\left\|\boldsymbol{\Gamma}_{i, j, k}^{\prime}\right\|_{\infty} \leq 1$.

Proposiçāo 3.2: Sob as suposições (1) - (6) temos que:

$\boldsymbol{E}\left(\hat{\beta}_{j k}^{(i)}-\beta_{j k}^{(i)}\right)^{2}=O\left(T^{-1}\right)$, para $i=\delta_{1}, \omega_{0}$

Prova. Da Proposição 3.1 e de (3.23) podemos inferir que existe um evento $\Omega_{0}$ tal que $P\left(\Omega_{0}\right) \geq 1-O\left(T^{-\lambda}\right)$ para $\lambda<\infty$ escolhido de maneira arbitrária e $\lambda$ grande, tal que

$$
\mathbb{E} \mathcal{I}\left(\Omega_{0}\right)\left(\left(\beta_{j k}^{(i)}-\hat{\beta}_{j k}^{(i)}\right)^{2}\right)=O\left(T^{-1}\right)
$$

em que $\mathcal{I}\left(\Omega_{0}\right)$ é o indicador de $\Omega_{0}$. De acordo com o Lema $A .3 .2, \operatorname{com} 0<\varrho_{1}<\varrho$ e pela Desigualdade de Hölder, temos que

$\boldsymbol{E} \mathcal{I}\left(\Omega_{0}^{c}\right)\left(\left(\beta_{j k}^{(i)}-\hat{\beta}_{j k}^{(i)}\right)^{2}\right) \leq \boldsymbol{E}\left(\left|\beta_{j k}^{(i)}-\hat{\beta}_{j k}^{(i)}\right|^{2+\varrho_{1}}\right)^{2 /\left(2+\varrho_{1}\right)} P\left(\Omega_{0}^{c}\right)^{1-2 /\left(2+\varrho_{1}\right)}=O\left(T^{-1}\right)$

o que completa a prova.

Proposição 3.3: Sob as suposições $(1)-(6)$ temos que: $\mathbb{P}\left( \pm\left(\hat{\beta}_{j k}^{(i)}-\beta_{j k}^{(i)}\right) / \sigma_{i j k} \geq x\right)=$ $(1-\Phi(x))(1+o(1))+O\left(T^{-\lambda}\right)$, para $i=\delta_{1}, \omega_{0}, j=0,1, \cdots, j^{*}$ e $k=0,1, \cdots, 2^{j^{*}-1}-$ 1.

Prova. Vimos que $\hat{\beta}_{j k}^{(i)}-\beta_{j k}^{(i)}=\Gamma_{i, j, k}^{\prime}\left(T_{1}+T_{2}+T_{3}\right)$ e que o comportamento assintótico de $\hat{\beta}_{j k}^{(i)}-\beta_{j k}^{(i)}$ é essencialmente determinado pelo comportamento de $\Gamma_{i j k}^{\prime} T_{1}$. De (3.24) e (3.25) temos que $\left\|T_{2}\right\|_{\infty}=O_{p}\left(2^{j^{*}} T^{-1} \log (T)\right)$ e $\left\|T_{3}\right\|_{\infty}=O_{p}\left(T^{-1 / 2-\tau}\right)$, para algum $\tau>0$. 
Como, para uma variável qualquer $Z_{T}, Z_{T}=O_{p}\left(g_{T}\right)$ significa que $\mathbb{P}\left\{\left|Z_{T}\right| \geq\right.$ $\left.M_{\epsilon} g_{T}\right\} \leq \epsilon$ ou seja, $Z_{T}$ é limitado em probabilidade por $g_{T} \cdot\left(T_{2}+T_{3}\right)=O_{p}\left(T^{-1 / 2-\tau}\right)$ implicando em $\boldsymbol{P}\left(\left|T_{2}+T_{3}\right| \geq M_{\epsilon} T^{-1 / 2-\tau}\right) \leq T^{-\lambda}$ para algum $\lambda>0$. Assim, podemos inferir que $\Gamma_{i, j, k}^{\prime}\left(T_{2}+T_{3}\right)=O_{p}\left(T^{-1 / 2-\kappa}\right)$, para algum $\kappa>0$.

O processo $\left\{X_{t, T}\right\}$ admite a representação $M A(\infty)$

$$
X_{t, T}=\sum_{s=0}^{\infty} \gamma_{t, T}^{X}(s) a_{t-s}
$$

com

$$
\sum_{s=0}^{\infty} \sup _{t, T}\left\{\left|\gamma_{t, T}^{X}(s)\right|\right\} \leq C_{1}, \quad \forall T
$$

e $a_{t}$ é ruído branco. Para detalhes, veja Künsch (1995).

Por (3.26) e pela Proposição 2.1, o processo $\left\{Y_{t, T}\right\}$ também admite a representação $M A(\infty)$ da forma,

$$
Y_{t, T}=\sum_{s=0}^{\infty} \gamma_{t, T}^{Y}(s) a_{t-s}+\epsilon_{t}
$$

com

$$
\sum_{r=0}^{\infty} \sup _{t, T}\left\{\left|\gamma_{t, T}^{Y}(r)\right|\right\} \leq C_{2}, \quad \forall T
$$

Usando a representação de $\left\{X_{t, T}\right\}$ e $\left\{Y_{t, T}\right\}$ dada por (3.26) e (3.27), respectivamente, e lembrando que $T_{1}=\left(\mathbb{E} \Upsilon^{\prime} \Upsilon\right)^{-1} \Upsilon^{\prime}$ e, com $\Upsilon$ dado por (3.15) e (3.16), isto é, $\Upsilon$ é construído a partir dos processos $\left\{X_{t, T}\right\}$ e $\left\{Y_{t, T}\right\}$ temos que $\Gamma_{i, j, k}^{\prime} T_{1}$ será:

$$
\begin{aligned}
\Gamma_{i, j, k}^{\prime} T_{1}= & \sum_{u=1}^{2^{j^{*}}}\left[\sum_{v=1}^{2^{j^{*}}}\left(\boldsymbol{E} \boldsymbol{\Upsilon}^{\prime} \boldsymbol{\Upsilon}\right)_{u, v}^{-1} \sum_{t=2}^{T} \phi_{j^{*}, v}(t)\left(\sum_{s=0}^{\infty} \gamma_{t-1, T}^{Y}(s) a_{t-1-s}+\epsilon_{t-1}\right) e_{t, T}+\right. \\
& \left.+\sum_{v=2^{j^{*}+1}}^{2^{j^{*}+1}}\left(\boldsymbol{E} \boldsymbol{\Upsilon}^{\prime} \boldsymbol{\Upsilon}\right)_{u, v}^{-1} \sum_{t=2}^{T} \phi_{j^{*}, v-2 j^{*}}(t)\left(\sum_{s=0}^{\infty} \gamma_{t, T}^{X}(s) a_{t-s}\right) e_{t, T}\right]\left(\Gamma_{i, j, k}^{\prime}\right)_{u}
\end{aligned}
$$


em que $\left(\Gamma_{i, j, k}^{\prime}\right)_{u}$ é o elemento da coluna $u$ da linha $v$ de $\Gamma^{\prime}$.

Como $e_{t, T}=\epsilon_{t}-\delta_{1}(t) \epsilon_{t-1}$ temos que

$$
\begin{aligned}
\Gamma_{i, j, k}^{\prime} T_{1}= & \sum_{t=2}^{T} \sum_{s=0}^{\infty} a_{t-1-s} \epsilon_{t}\left(\gamma_{t-1, T}^{Y}(s)\left[1-\delta_{1}(t)\right] \sum_{v=1}^{2^{j^{*}}} \phi_{j^{*}, v}(t) \sum_{u=1}^{2^{j^{*}}}\left(\boldsymbol{E} \boldsymbol{\Upsilon}^{\prime} \boldsymbol{\Upsilon}\right)_{u, v}^{-1}\left(\boldsymbol{\Gamma}_{i, j, k}^{\prime}\right)_{u}\right)+ \\
& +\sum_{t=2}^{T} \sum_{s=0}^{\infty} \epsilon_{t-1} \epsilon_{t}\left(\left[1-\delta_{1}(t)\right] \sum_{v=1}^{2^{j^{*}}} \phi_{j^{*}, v}(t) \sum_{u=1}^{2^{j^{*}}}\left(\boldsymbol{E} \boldsymbol{\Upsilon}^{\prime} \boldsymbol{\Upsilon}\right)_{u, v}^{-1}\left(\boldsymbol{\Gamma}_{i, j, k}^{\prime}\right)_{u}\right)+ \\
+ & \sum_{t=2}^{T} \sum_{s=0}^{\infty} a_{t-s} \epsilon_{t}\left(\gamma_{t, T}^{X}(s)\left[1-\delta_{1}(t)\right] \sum_{v=2^{j^{*}+1}}^{j^{j^{*}+1}} \phi_{j^{*}, v-2^{j^{*}}}(t) \sum_{u=1}^{2^{j^{*}}}\left(\boldsymbol{E} \boldsymbol{\Upsilon}^{\prime} \boldsymbol{\Upsilon}\right)_{u, v}^{-1}\left(\boldsymbol{\Gamma}_{i, j, k}^{\prime}\right)_{u}\right)
\end{aligned}
$$

que pode ser escrito como:

$$
\begin{aligned}
\Gamma_{i, j, k}^{\prime} T_{1}= & \sum_{s=0}^{\infty} \sum_{t=2}^{T} a_{t-1-s} \epsilon_{t} W_{t}^{Y}(1, s)+\sum_{s=0}^{\infty} \sum_{t=2}^{T} \epsilon_{t} \epsilon_{t-1} W_{t}^{Y}(2, s)+ \\
& +\sum_{s=0}^{\infty} \sum_{t=2}^{T} a_{t-s} \epsilon_{t} W_{t}^{X}(1, s)
\end{aligned}
$$

em que

$$
\begin{aligned}
& W_{t}^{Y}(1, s)=\gamma_{t-1, T}^{Y}(s)\left[1-\delta_{1}(t)\right] \sum_{v=1}^{2^{j^{*}}} \phi_{j^{*}, v}(t) \sum_{u=1}^{2^{j^{*}}}\left(\boldsymbol{E} \Upsilon^{\prime} \Upsilon\right)_{u, v}^{-1}\left(\boldsymbol{\Gamma}_{i, j, k}^{\prime}\right)_{u} \\
& W_{t}^{Y}(2, s)=\left[1-\delta_{1}(t)\right] \sum_{v=1}^{2^{j^{*}}} \phi_{j^{*}, v}(t) \sum_{u=1}^{2^{j^{*}}}\left(\mathbb{E} \boldsymbol{\Upsilon}^{\prime} \Upsilon\right)_{u, v}^{-1}\left(\boldsymbol{\Gamma}_{i, j, k}^{\prime}\right)_{u} \\
& W_{t}^{X}(1, s)=\gamma_{t, T}^{X}(s)\left[1-\delta_{1}(t)\right] \sum_{v=2^{j^{*}+1}}^{2^{j^{*}+1}} \phi_{j^{*}, v-2^{j^{*}}}(t) \sum_{u=1}^{2^{j^{*}}}\left(\mathbb{E} \Upsilon^{\prime} \Upsilon\right)_{u, v}^{-1}\left(\boldsymbol{\Gamma}_{i, j, k}^{\prime}\right)_{u}
\end{aligned}
$$

Usando os fatos que $\sup _{v}\left\{\left|\left(\boldsymbol{\Gamma}_{i, j, k}\right)_{v}\right|\right\}=O\left(2^{-\left(j^{*}-j\right) / 2}\right), \sum_{v}\left|\left(\Gamma_{i, j, k}\right)_{v}\right|=O\left(2^{\left(j^{*}-j\right) / 2}\right)$ e $\sum_{t} \phi_{j^{*}, v}(t / T)=O\left(2^{-j^{*} / 2} T\right)$ obtemos que:

$$
\begin{aligned}
& \left\|W_{t}^{Y}(1, s)\right\|_{\infty}=O\left(T^{-1} \sup _{t}\left\{\left|\gamma_{t-1}^{Y}(s)\right|\right\} 2^{j / 2}\right), \\
& \left\|W_{t}^{-Y}(2, s)\right\|_{\infty}=O\left(T^{-1} 2^{j / 2}\right) \quad \mathrm{e} \\
& \left\|W_{t}^{X}(1, s)\right\|_{\infty}=O\left(T_{t}^{-1} \sup _{t}\left\{\left|\gamma_{t}^{X}(s)\right|\right\} 2^{j / 2}\right) .
\end{aligned}
$$


Temos ainda que

$$
\sum_{i} \sup _{j}\left\{\left|W^{Y}(1, s)_{i j}\right|\right\}=\sum_{i} \sup _{j}\left\{\left|W^{X}(1, s)_{i j}\right|\right\}=\sum_{i} \sup _{j}\left\{\left|W^{Y}(2, s)_{i j}\right|\right\}=O\left(2^{-j / 2}\right)
$$

Pela Suposição 3, a soma em $s$ não afeta as taxas de (3.28) e (3.29).

Assim, o produto $\Gamma_{i, j, k}^{\prime} T_{1}$ pode ser escrito como uma soma de formas quadráticas,

$$
\Gamma_{i, j, k}^{\prime} T_{1}=\mathbf{a} \mathbf{W}^{\mathbf{Y}} \boldsymbol{\epsilon}+\boldsymbol{\epsilon} \mathbf{W}^{\mathbf{Y}} \boldsymbol{\epsilon}+\mathbf{a} \mathbf{W}^{\mathbf{X}} \boldsymbol{\epsilon}
$$

Como a e $\epsilon$ são independentes, a é o ruído branco formador do processo de entrada $\left\{X_{t, T}\right\}$ e $\epsilon$ é o ruído branco da função de transferência, e $\mathbf{W}^{\mathbf{Y}}$ satisfaz as condições do Lema $A .2 .1$, isto é, $\tilde{W}^{\mathbf{Y}}=O\left(2^{-j / 2}\right)$ e $\left\|\mathbf{W}^{\mathbf{Y}}\right\|_{\infty}=O\left(T^{-1} 2^{j / 2}\right)$, seguindo os mesmos passos que Dahlhaus et al. (1999), temos que,

$$
\left|\operatorname{cum}_{n}\left(\Gamma_{i, j, k}^{\prime} T_{1}\right)\right|=\left|\operatorname{cum}_{n}\left(\epsilon \mathbf{W}^{\mathbf{Y}} \boldsymbol{\epsilon}\right)\right| \leq C^{n} T^{-1}(n !)^{2+2 \gamma}\left(T^{-1} 2^{j / 2}\right)^{n-2} .
$$

Utilizando o Lema $A .2 .2$,

$$
\mathbb{P}\left( \pm\left(\Gamma_{i, j, k}^{\prime} T_{1}\right) / \sigma_{i j k} \geq x\right)=(1-\Phi(x))(1+o(1))
$$

uniformemente em $0 \leq x \leq \kappa_{T}, \kappa_{T}$ é de mesma ordem que $T^{\nu}$, para algum $\nu>0$.

Lembrando que $\hat{\beta}_{j k}^{(i)}-\beta_{j k}^{(i)}=\Gamma_{i, j, k}^{\prime} T_{1}+O_{p}\left(T^{-1 / 2-\kappa}\right)$ para algum $\kappa>0$, temos que

$$
\begin{array}{r}
\boldsymbol{P}\left( \pm\left(\boldsymbol{\Gamma}_{i, j, k}^{\prime} T_{1}\right) / \sigma_{i j k} \geq x+C T^{-\kappa}\right)-C T^{-\lambda} \leq \mathbb{P}\left( \pm\left(\hat{\beta}_{j k}^{(i)}-\beta_{j k}^{(i)}\right) / \sigma_{i j k} \geq x\right) \leq \\
P\left( \pm\left(\Gamma_{i, j, k}^{\prime} T_{1}\right) / \sigma_{i j k} \geq x-C T^{-\kappa}\right)+C T^{-\lambda}
\end{array}
$$

o que implica em

$$
\begin{array}{r}
\mathbb{P}\left( \pm\left(\hat{\beta}_{j k}^{(i)}-\beta_{j k}^{(i)}\right) / \sigma_{i j k} \geq x\right)=[1-\Phi(x)](1+o(1))+ \\
O\left[\left|\Phi(x)-\Phi\left(x+C T^{-\kappa}\right)\right|\right]+O\left[\left|\Phi(x)-\Phi\left(x-C T^{-\kappa}\right)\right|\right]+O\left(T^{-\lambda}\right) .
\end{array}
$$


Seja $c>1$, fixo. Para $x \leq c$ temos

$$
\left|\Phi(x)-\Phi\left(x+C T^{-\kappa}\right)\right| \leq C T^{-\kappa} \phi(0)=o(1-\Phi(x))
$$

em que $\phi(x)$ e $\Phi(x)$ são os valores da função densidade probabilidade normal padrão e da função distribuição acumulada, respectivamente.

Para $c<x \leq C(2 \lambda \log (T))^{1 / 2}$ obtemos, pela fórmula da Razão de Mills (Johnson et al. (1970)), que

$$
\begin{aligned}
\left|\Phi(x)-\Phi\left(x+C T^{-\kappa}\right)\right| & \leq C T^{-\kappa} \phi(x) \\
& \leq C T^{-\kappa} x\left(1-\frac{1}{x^{2}}\right)^{-1}(1-\Phi(x)) \\
& \leq C T^{-\kappa} x\left(1-\frac{1}{c^{2}}\right)^{-1}(1-\Phi(x))=o(1-\Phi(x)) .
\end{aligned}
$$

O terceiro termo de (3.30) é tratado da mesma maneira.

Para $x>C(2 \lambda \log (T))^{1 / 2}$ temos

$$
P\left( \pm\left(\hat{\beta}_{j k}^{(i)}-\beta_{j k}^{(i)}\right) / \sigma_{i j k} \geq x\right)=O\left(T^{-\lambda}\right)=(1-\Phi(x))(1+o(1))+O\left(T^{-\lambda}\right)
$$

o que completa a prova.

\subsection{Estimadores do Segundo Estágio.}

No segundo estágio ajustamos o modelo descrito em (3.3) e a equação matricial do segundo estágio é análoga a do primeiro estágio (3.7):

$$
\mathbf{Y}=\left[\begin{array}{lllllllll}
\Phi_{\hat{Y}_{(-1)}} & \Psi_{\dot{Y}_{(-1)}}^{(0)} & \cdots & \Psi_{\hat{Y}_{(-1)}}^{\left(j^{*}-1\right)} & \vdots & \Phi_{X} & \Psi_{X} & \cdots & \Psi_{X}^{\left(j^{*}-1\right)}
\end{array}\right]\left[\begin{array}{l}
\beta^{\left(\delta_{1}\right)} \\
\ldots \\
\boldsymbol{\beta}^{\left(\omega_{0}\right)}
\end{array}\right]+\mathbf{n}
$$


em que $\mathbf{n}$ é o vetor de erros aleatórios, $\mathbf{n}=\left[\begin{array}{llll}n_{2} & n_{3} & \ldots & n_{T}\end{array}\right]^{\prime}$. Mais adiante mostraremos que $\left\{n_{t}\right\}$ é um processo média móveis de ordem 2.

As diferenças são que a matriz $\Phi_{Y_{(-1)}}$ citada em (3.5) agora será substituída por

$$
\boldsymbol{\Phi}_{\hat{Y}_{(-1)}}=\left[\begin{array}{c}
\phi_{00}\left(\frac{2}{T}\right) \hat{Y}_{1, T} \\
\phi_{00}\left(\frac{3}{T}\right) \hat{Y}_{2, T} \\
\vdots \\
\phi_{00}\left(\frac{T}{T}\right) \hat{Y}_{T-1, T}
\end{array}\right]
$$

e (3.6) será substituída neste estágio por

$$
\Psi_{\hat{Y}_{(-1)}^{(m)}}^{(m)}=\left[\begin{array}{cccc}
\psi_{m 0}\left(\frac{2}{T}\right) \hat{Y}_{1, T} & \psi_{m 1}\left(\frac{2}{T}\right) \hat{Y}_{1, T} & \cdots & \psi_{m, 2^{m}-1}\left(\frac{2}{T}\right) \hat{Y}_{1, T} \\
\psi_{m 0}\left(\frac{2}{T}\right) \hat{Y}_{2, T} & \psi_{m 1}\left(\frac{2}{T}\right) \hat{Y}_{2, T} & \cdots & \psi_{m, 2^{m}-1}\left(\frac{2}{T}\right) \hat{Y}_{2, T} \\
\vdots & \vdots & \ddots & \vdots \\
\psi_{m 0}\left(\frac{T}{T}\right) \hat{Y}_{T-1, T} & \psi_{m 1}\left(\frac{T}{T}\right) \hat{Y}_{T-1, T} & \cdots & \psi_{m, 2^{m}-1}\left(\frac{T}{T}\right) \hat{Y}_{T-1, T}
\end{array}\right]
$$

lembrando que $\hat{Y}_{t, T}$ é o valor ajustado no primeiro estágio, $\hat{Y}_{t, T}=\hat{\delta}_{1}^{(L)}(t) Y_{t-1, T}+$ $\hat{\omega}_{0}^{(L)}(t) X_{t, T}$ para $t=2,3, \cdots, T$.

Agora vamos escrever $n_{t, T}$ em função de $\epsilon_{t}$.

$$
\begin{aligned}
n_{t, T} & =Y_{t, T}-\delta_{1}(t) \hat{Y}_{t-1, T}-\omega_{0}(t) X_{t, T} \\
& =Y_{t, T}-\delta_{1}(t)\left[Y_{t-1, T}-e_{t-1}\right]-\omega_{0}(t) X_{t, T} \\
& =Y_{t, T}-\delta_{1}(t) Y_{t-1, T}-\omega_{0}(t) X_{t, T}+\delta_{1}(t) e_{t-1} \\
& =Y_{t, T}-\delta_{1}(t) Y_{t-1, T}-\omega_{0}(t) X_{t, T}+\delta_{1}(t)\left[\epsilon_{t-1}-\delta_{1}(t) \epsilon_{t-2}\right] \\
& =Y_{t, T}-\delta_{1}(t) Y_{t-1, T}-\omega_{0}(t) X_{t, T}+\delta_{1}(t) \epsilon_{t-1}-\delta_{1}^{2}(t) \epsilon_{t-2}
\end{aligned}
$$

mas, $Y_{t, T}=\frac{\omega_{0}(t)}{1-\delta_{1}(t) B} X_{t, T}+\epsilon_{t}$ então, $Y_{t, T}=\delta_{1}(t) Y_{t-1, T}+\omega_{0}(t) X_{t, T}+\epsilon_{t}-\delta_{1}(t) \epsilon_{t-1} 0$ que resulta em, $n_{t, T}=\epsilon_{t}-\delta_{1}^{2}(t) \epsilon_{t-2}$. 
O estimador de Mínimos Quadrados, neste estágio, é dado por:

$$
\left[\begin{array}{c}
\tilde{\boldsymbol{\beta}}^{\left(\delta_{1}\right)} \\
\tilde{\boldsymbol{\beta}}^{\left(\omega_{0}\right)}
\end{array}\right]=\left[\begin{array}{cc}
\Psi_{\hat{Y}_{(-1)}}^{\prime} \Psi_{\hat{Y}_{(-1)}} & \Psi_{\hat{Y}_{(-1)}}^{\prime} \Psi_{X} \\
\Psi_{X}^{\prime} \Psi_{\hat{Y}_{(-1)}} & \Psi_{X}^{\prime} \Psi_{X}
\end{array}\right]^{-1}\left[\begin{array}{c}
\Psi_{\hat{Y}_{(-1)}}^{\prime} \mathbf{Y} \\
\Psi_{X}^{\prime} \mathbf{Y}
\end{array}\right]
$$

\subsubsection{Propriedades Estatísticas}

Da mesma maneira que no primeiro estágio, escrevemos os estimadores no espaço $V_{j^{*}}$, isto é, vamos encontrar $\tilde{\beta}_{j k}^{(i)}$ a partir de $\tilde{\zeta}$. O modelo de regressão que estamos ajustando é, semelhante ao da equação (3.12).

$$
Y_{t, T}=\sum_{i=1}^{2^{j^{*}}} \zeta_{j^{*}, i}^{\left(\delta_{1}\right)} \phi_{j^{*}, i}\left(\frac{t}{T}\right) \hat{Y}_{t-1, T}(t)+\sum_{i=1}^{2^{j^{*}}} \zeta_{j^{*}, i}^{\left(\omega_{0}\right)} \phi_{j^{*}, i}\left(\frac{t}{T}\right) X_{t, T}+\hat{\gamma}_{t, T}
$$

em que

$$
\hat{\gamma}_{t, T}=\sum_{j \geq j^{*}} \sum_{k \in I_{j}} \beta_{j k}^{\left(\delta_{1}\right)} \psi_{j k}\left(\frac{t}{T}\right) \hat{Y}_{t-1, T}+\sum_{j \geq j^{*}} \sum_{k \in I_{j}} \beta_{j k}^{\left(\omega_{0}\right)} \psi_{j k}\left(\frac{t}{T}\right) X_{t, T}+n_{t, T}
$$

Escrevendo a equação (3.31) na forma matricial temos:

$$
\mathrm{Y}=\left[\begin{array}{lll}
\varphi_{\hat{Y}_{(-1)}} & \vdots & \varphi_{X}
\end{array}\right]\left[\begin{array}{c}
\boldsymbol{\zeta}^{\left(\delta_{1}\right)} \\
\cdots \\
\zeta^{\left(\omega_{0}\right)}
\end{array}\right]+\hat{\gamma}
$$

com

$$
\varphi_{\hat{Y}_{(-1)}}=\left[\begin{array}{cccc}
\phi_{j^{*}, 1}\left(\frac{2}{T}\right) \hat{Y}_{1, T} & \phi_{j^{*}, 2}\left(\frac{2}{T}\right) \hat{Y}_{1, T} & \cdots & \phi_{j^{*}, 2^{*}}\left(\frac{2}{T}\right) \hat{Y}_{1, T} \\
\phi_{j^{*}, 1}\left(\frac{3}{T}\right) \hat{Y}_{2, T} & \phi_{j^{*}, 2}\left(\frac{3}{T}\right) \hat{Y}_{2, T} & \cdots & \phi_{j^{*}, 2^{*}}\left(\frac{2}{T}\right) \hat{Y}_{2, T} \\
\vdots & \vdots & \ddots & \vdots \\
\phi_{j^{*}, 1}\left(\frac{T}{T}\right) \hat{Y}_{T-1, T} & \phi_{j^{*}, 2}\left(\frac{T}{T}\right) \hat{Y}_{T-1, T} & \cdots & \phi_{j^{*}, 2^{*}}\left(\frac{T}{T}\right) \hat{Y}_{T-1, T}
\end{array}\right]
$$

e

$$
\varphi_{X}=\left[\begin{array}{cccc}
\phi_{j^{*}, I}\left(\frac{2}{T}\right) X_{2, T} & \phi_{j^{*}, 2}\left(\frac{2}{T}\right) X_{2, T} & \cdots & \phi_{j^{*}, 2^{*}}\left(\frac{2}{T}\right) X_{2, T} \\
\phi_{j^{*}, 1}\left(\frac{3}{T}\right) X_{3, T} & \phi_{j^{*}, 2}\left(\frac{3}{T}\right) X_{3 . T} & \cdots & \phi_{j^{*}, 2^{*}}\left(\frac{3}{T}\right) X_{3, T} \\
\vdots & \vdots & \ddots & \vdots \\
\phi_{j^{*}, 1}\left(\frac{T}{T}\right) X_{T, T} & \phi_{j^{*}, 2}\left(\frac{T}{T}\right) X_{T, T} & \cdots & \phi_{j^{*}, 2^{*}}\left(\frac{T}{T}\right) X_{T, T}
\end{array}\right] \text {. }
$$


Segue-se analogamente às equações (3.10), que

$$
\left[\begin{array}{l}
\tilde{\zeta}^{\left(\delta_{1}\right)} \\
\tilde{\zeta}^{\left(\omega_{0}\right)}
\end{array}\right]=\left[\begin{array}{ll}
\varphi_{Y_{(-1)}}^{\prime} \varphi_{\hat{Y}_{(-1)}} & \varphi_{\hat{Y}_{(-1)}}^{\prime} \varphi_{X} \\
\varphi_{X}^{\prime} \varphi_{\hat{Y}_{(-1)}} & \varphi_{X}^{\prime} \varphi_{X}
\end{array}\right]^{-1}\left[\begin{array}{l}
\varphi_{\hat{Y}_{(-1)}}^{\prime} \mathbf{Y} \\
\varphi_{X}^{\prime} \mathbf{Y}
\end{array}\right] .
$$

De uma forma mais simplificada temos

$$
Y=\hat{\Upsilon}^{\prime} \zeta+\hat{\gamma}
$$

$\operatorname{com} \hat{\boldsymbol{\Upsilon}}=\left[\boldsymbol{\varphi}_{\hat{Y}_{(-1)}} \boldsymbol{\varphi}_{X}\right]$ e $\boldsymbol{\zeta}=\left(\begin{array}{l}\boldsymbol{\zeta}^{\left(\delta_{1}\right)} \\ \boldsymbol{\zeta}^{\left(\omega_{0}\right)}\end{array}\right)$.

O estimador de $\boldsymbol{\zeta}$ é $\tilde{\zeta}$, dado por

$$
\tilde{\zeta}=\left(\hat{\Upsilon}^{\prime} \hat{\Upsilon}\right)^{-1} \hat{\Upsilon}^{\prime} \mathbf{Y}
$$

De (3.32) e (3.33) temos

$$
\tilde{\zeta}=\left(\hat{\Upsilon}^{\prime} \hat{\Upsilon}\right)^{-1} \hat{\Upsilon}^{\prime}(\hat{\Upsilon} \boldsymbol{\zeta}+\hat{\gamma}) .
$$

Se escrevermos $\hat{\gamma}=\mathbf{n}+\hat{\mathbf{S}}$, obtemos

$$
\tilde{\zeta}=\left(\hat{\Upsilon}^{\prime} \hat{\boldsymbol{\Upsilon}}\right)^{-1} \hat{\Upsilon}^{\prime}(\hat{\Upsilon} \zeta+\mathbf{n}+\hat{\mathbf{S}})
$$

onde a matriz $\hat{\mathbf{S}}$, de ordem $(T-1) \times 1$, é dada por

$$
\hat{\mathbf{S}}=\left[\begin{array}{c}
\sum_{j \geq j^{*}} \sum_{k \in \mathcal{I}_{j}} \beta_{j k} \psi_{j k}\left(\frac{2}{T}\right) \hat{Y}_{1, T}+\sum_{j \geq j^{*}} \sum_{k \in \mathcal{I}_{j}} \beta_{j k} \psi_{j k}\left(\frac{2}{T}\right) X_{2, T} \\
\sum_{j \geq j^{*}} \sum_{k \in \mathcal{I}_{j}} \beta_{j k} \psi_{j k}\left(\frac{3}{T}\right) \hat{Y}_{2, T}+\sum_{j \geq j^{*}} \sum_{k \in \mathcal{I}_{j}} \beta_{j k} \psi_{j k}\left(\frac{3}{T}\right) X_{3, T} \\
\vdots \\
\sum_{j \geq j^{*}} \sum_{k \in \mathcal{I}_{j}} \beta_{j k} \psi_{j k}\left(\frac{T}{T}\right) \hat{Y}_{T-1, T}+\sum_{j \geq j^{*}} \sum_{k \in \mathcal{I}_{j}} \beta_{j k} \psi_{j k}\left(\frac{T}{T}\right) X_{T, T}
\end{array}\right] .
$$

Note que

$$
\varphi_{\hat{Y}(-1)}^{\prime} \mathbf{n}=\left[\begin{array}{c}
\sum_{t=2}^{T} \phi_{j^{*}, 1}\left(\frac{t}{T}\right) \hat{Y}_{t-1, T} n_{t} \\
\sum_{t=2}^{T} \phi_{j^{*}, 2}\left(\frac{t}{T}\right) \hat{Y}_{t-1, T} n_{t} \\
\vdots \\
\sum_{t=2}^{T} \phi_{j^{*}, 2^{j^{*}}}\left(\frac{t}{T}\right) \hat{Y}_{t-1, T} n_{t}
\end{array}\right],
$$




$$
\boldsymbol{\varphi}_{X^{\prime}}^{\prime} \mathbf{n}=\left[\begin{array}{c}
\sum_{t=2}^{T} \phi_{j^{*}, 1}\left(\frac{t}{T}\right) X_{t, T} n_{t} \\
\sum_{t=2}^{T} \phi_{j^{*}, 2}\left(\frac{t}{T}\right) X_{t, T} n_{t} \\
\vdots \\
\sum_{t=2}^{T} \phi_{j^{*}, 2^{*}}\left(\frac{t}{T}\right) X_{t, T} n_{t}
\end{array}\right]
$$

Proposição 3.4: O processo $\hat{Y}_{t, T}$ é localmente estacionário, de acordo com a Definição 2.1 .

Prova.

$$
\hat{Y}_{t, T}=\hat{\delta}_{1}^{(L)}(t / T) Y_{t-1, T}+\hat{\omega}_{0}^{(L)}(t / T) X_{t, T}
$$

em que, usando a definição 2.1 , temos que,

$$
\begin{aligned}
Y_{t-1, T}= & \mu_{Y}\left(\frac{t-1}{T}\right)+\int_{-\pi}^{\pi} e^{i \lambda(t-1)} A_{Y}\left(\frac{t-1}{T}, \lambda\right) d \xi(\lambda)+ \\
& +\int_{-\pi}^{\pi} e^{i \lambda(t-1)} \frac{1}{\sqrt{2 \pi}} d \zeta(\lambda)
\end{aligned}
$$

e

$$
X_{t, T}=\mu_{X}\left(\frac{t}{T}\right)+\int_{-\pi}^{\pi} e^{i \lambda(t)} A_{X}\left(\frac{t}{T}, \lambda\right) d \xi(\lambda)
$$

Assim, substituindo (3.36) e (3.37) em (3.35) temos,

$$
\begin{aligned}
\hat{Y}_{t, T}= & \hat{\delta}_{1}\left(\frac{t-1}{T}\right) \mu_{Y}\left(\frac{t-1}{T}\right)+\int_{-\pi}^{\pi} e^{i \lambda t} e^{-i \lambda} \hat{\delta}_{1}^{(L)}\left(\frac{t-1}{T}\right) A_{Y}\left(\frac{t-1}{T}, \lambda\right) d \xi(\lambda)+ \\
& +\int_{-\pi}^{\pi} e^{i \lambda(t-1)} \hat{\delta}_{1}^{(L)}\left(\frac{t-1}{T}\right) \frac{1}{\sqrt{2 \pi}} d \zeta(\lambda)+\hat{\omega}_{0}^{(L)}\left(\frac{t}{T}\right) \mu_{X}\left(\frac{t}{T}\right)+ \\
& +\int_{-\pi}^{\pi} e^{i \lambda t} \hat{\omega}_{0}^{(L)}\left(\frac{t}{T}\right) A_{X}\left(\frac{t}{T}, \lambda\right) d \xi(\lambda) \\
= & \hat{\delta}_{1}^{(L)}\left(\frac{t-1}{T}\right) \mu_{Y}\left(\frac{t-1}{T}\right)+\hat{\omega}_{0}^{(L)}\left(\frac{t}{T}\right) \mu_{X}\left(\frac{t}{T}\right)+ \\
& +\int_{-\pi}^{\pi} e^{i \lambda t}\left[e^{-i \lambda} \hat{\delta}_{1}^{(L)}\left(\frac{t-1}{T}\right) A_{Y}\left(\frac{t-1}{T}, \lambda\right)+\hat{\omega}_{0}^{(L)}\left(\frac{t}{T}\right) A_{X}\left(\frac{t}{T}, \lambda\right)\right] d \xi(\lambda)+ \\
& +\int_{-\pi}^{\pi} e^{i \lambda(t-1)} \hat{\delta}_{1}^{(L)}\left(\frac{t-1}{T}\right) \frac{1}{\sqrt{2 \pi}} d \zeta(\lambda) .
\end{aligned}
$$


O que completa a prova.

Conjecturamos que as Propriedades 3.2 e 3.3 também valem para o segundo estágio, embora não tenhamos provados analiticamente.

\subsection{Intervalos}

Demonstramos que os coeficientes empíricos do primeiro estágio têm distribuição assintoticamente normal. Para a curva limiarizada, isto é, antes de aplicarmos a transformada inversa de ondaletas, fazemos uma limiarização, que não é uma transformação linear implicando que não temos como determinar a distribuição para todos os pontos da curva verdadeira.

Usando o procedimento bootstrap não paramétrico, apresentado em Efron et al. (1993) podemos construir intervalos para as curvas verdadeiras.

Com as estimativas das curvas para os valores de $t=1,2,3, \cdots, T$, calculamos o valor ajustado da série de saída e, em seguida, a série de resíduos, através da diferença entre o valor real e o valor ajustado da série de saída, $r e_{1}, r e_{2}, r e_{3}, \cdots, r e_{T}$.

Com esses valores de resíduos fazemos uma amostragem, com reposição e de tamanho $T$, formando os resíduos $r e_{1}^{*}, r e_{2}^{*}, r e_{3}^{*}, \cdots, r e_{T}^{*}$ que são somados aos valores ajustados da série de saída, obtemos daí a primeira réplica bootstrap, $Y_{1}^{*}, Y_{2}^{*}, Y_{3}^{*}$, $\cdots, Y_{T}^{*}$. Com esta série ajustamos novamente o modelo e obtemos novas estimativas $\operatorname{de} \hat{\boldsymbol{\beta}}^{(\boldsymbol{\delta})}, \hat{\boldsymbol{\beta}}^{\left(\boldsymbol{\delta}^{(L)}\right)}, \tilde{\boldsymbol{\beta}}^{(\boldsymbol{\delta})}, \tilde{\boldsymbol{\beta}}^{\left(\boldsymbol{\delta}^{(L)}\right)}, \hat{\boldsymbol{\beta}}^{(\boldsymbol{\omega})}, \hat{\boldsymbol{\beta}}^{\left(\boldsymbol{\omega}^{(L)}\right)}, \tilde{\boldsymbol{\beta}}^{(\boldsymbol{\omega})}$ e $\tilde{\boldsymbol{\beta}}^{\left(\boldsymbol{\omega}^{(L)}\right)}$. Para cada passo reconstruímos as funções $\hat{\delta}, \hat{\delta}^{(L)}, \tilde{\delta}, \tilde{\delta}^{(L)}, \hat{\omega}, \hat{\omega}^{(L)}, \tilde{\omega}$ e $\tilde{\omega}^{(L)}$.

Repetimos os passos de amostragem e estimação $\mathbb{B}$ vezes. Temos agora $\mathbb{B}$ curvas bootstrap para cada coeficiente da função de transferência e, com isso construímos 
intervalos de confiança baseados nos percentis para cada ponto.

\subsection{Generalizações}

Consideremos agora um modelo de função de transferência mais geral, isto é, com valores $b, r$ e $s$ quaisquer.

O modelo será

$$
Y_{t, T}=\frac{\omega_{0}\left(\frac{t}{T}\right) B^{b}+\omega_{1}\left(\frac{t}{T}\right) B^{b+1}+\cdots+\omega_{s}\left(\frac{t}{T}\right) B^{b+s}}{1-\delta_{1}\left(\frac{t}{T}\right) B-\delta_{2}\left(\frac{t}{T}\right) B^{2}-\cdots-\delta_{r}\left(\frac{t}{T}\right) B^{r}} X_{t, T}+\epsilon_{t} .
$$

Então, temos,

$$
\begin{aligned}
Y_{t, T}= & \delta_{1}\left(\frac{t}{T}\right) Y_{t-1, T}-\delta_{2}\left(\frac{t}{T}\right) Y_{t-1, T}-\cdots-\delta_{r}\left(\frac{t}{T}\right) Y_{t-r, T}+ \\
& +\omega_{0}\left(\frac{t}{T}\right) X_{t-b, T}+\omega_{1}\left(\frac{t}{T}\right) X_{t-b-1, T}+\cdots+\omega_{s}\left(\frac{t}{T}\right) X_{t-b-s, T}+\epsilon_{t}+ \\
& -\delta_{1}\left(\frac{t}{T}\right) \epsilon_{t-1}-\delta_{2}\left(\frac{t}{T}\right) \epsilon_{t-2}-\cdots-\delta_{r}\left(\frac{t}{T}\right) \epsilon_{t-r}
\end{aligned}
$$

ou seja,

$$
\begin{aligned}
Y_{t, T}= & \delta_{1}\left(\frac{t}{T}\right)\left[Y_{t-1, T}-\epsilon_{t-1}\right]+\delta_{2}\left(\frac{t}{T}\right)\left[Y_{t-2, T}-\epsilon_{t-2}\right]+\cdots+ \\
& +\delta_{r}\left(\frac{t}{T}\right)\left[Y_{t-r, T}-\epsilon_{t-r}\right]+\omega_{0}\left(\frac{t}{T}\right) X_{t-b, T}+ \\
& +\omega_{1}\left(\frac{t}{T}\right) X_{t-b-1 . T}+\cdots+\omega_{s}\left(\frac{t}{T}\right) X_{t-b-s}+\epsilon_{t}
\end{aligned}
$$

\subsubsection{Estimadores do Primeiro Estágio}

No primeiro estágio fazemos a regressão de $Y_{t . T}$ sobre $Y_{t-1, T}, \cdots, Y_{t-r, T}$ e $X_{t-b, T}$, $X_{t-b-1 . T}, \cdots, X_{t-b-s, T}$ 
Matricialmente temos:

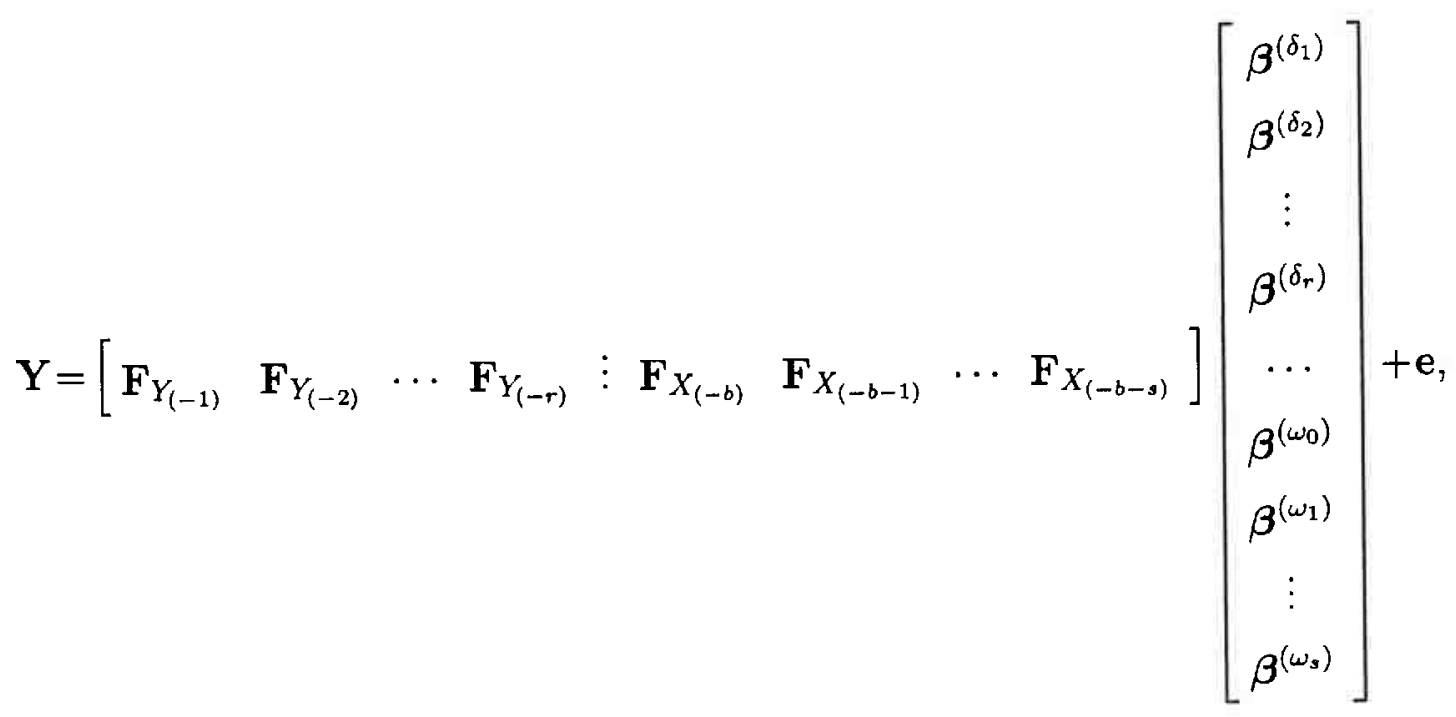

em que, para $0 \leq m \leq j^{*}$,

$$
\mathbf{F}_{Y_{(-m)}}=\left[\begin{array}{llll}
\Phi_{Y_{(-m)}} & \Psi_{Y_{(-m)}}^{(0)} & \cdots & \Psi_{Y_{(-m)}}^{\left(j^{*}-1\right)}
\end{array}\right]
$$

e

$$
\mathbf{F}_{X_{(-m)}}=\left[\begin{array}{llll}
\boldsymbol{\Phi}_{X_{(-m)}} & \Psi_{X_{(-m)}} & \cdots & \Psi_{X_{(-m)}}^{\left(j^{*}-1\right)}
\end{array}\right]
$$

e é o vetor de erros aleatórios e é um processo médias móveis de ordem $r$.

O estimador do primeiro estágio será:

$$
\hat{\mathbf{B}}=\Xi^{-1} \Xi_{x y}
$$

em que

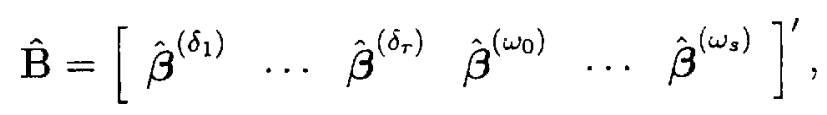




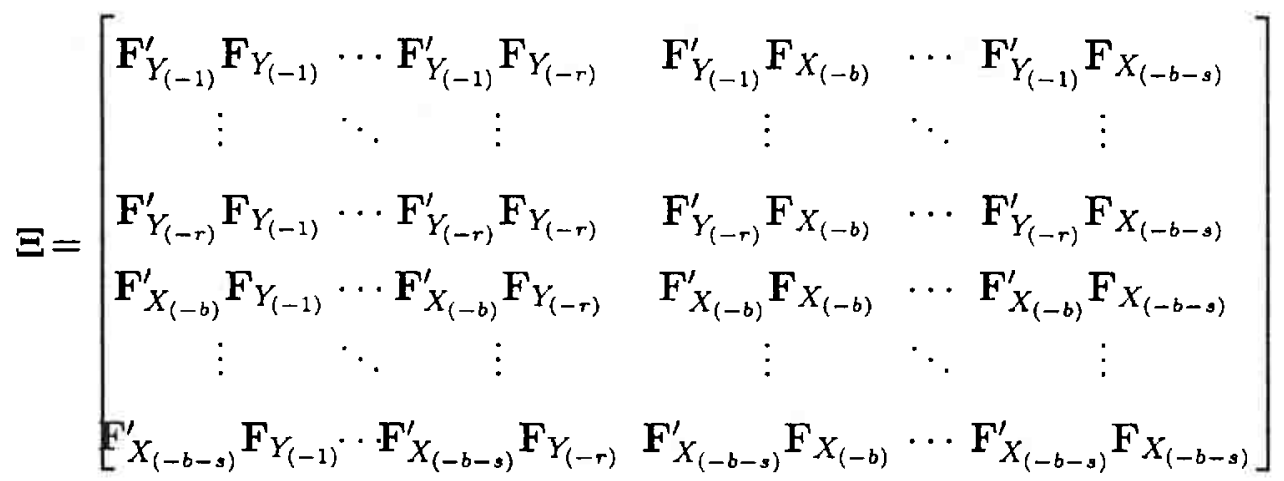

$\mathrm{e}$

$$
\boldsymbol{\Xi}_{x y}=\left[\begin{array}{lllllllll}
\mathbf{F}_{Y_{(-1)}}^{\prime} \mathbf{Y} & \cdots & \mathbf{F}_{Y_{(-r)}}^{\prime} \mathbf{Y} & \mathbf{F}_{X_{(-b)}}^{\prime} \mathbf{Y} & \cdots & \mathbf{F}_{X_{(-b-s)}^{\prime}}^{\prime} \mathbf{Y}
\end{array}\right]^{\prime}
$$

\section{Propriedades Estatísticas}

O vetor $\hat{\mathbf{B}}=\left[\hat{\boldsymbol{\beta}}^{\left(\delta_{1}\right)}, \cdots, \hat{\boldsymbol{\beta}}^{\left(\delta_{r}\right)}, \hat{\boldsymbol{\beta}}^{\left(\omega_{0}\right)}, \cdots, \hat{\boldsymbol{\beta}}^{\left(\omega_{s}\right)}\right]^{\prime}$ é encontrado através da transformação $\Gamma^{\prime} \hat{\zeta} \operatorname{com} \hat{\zeta}=\left[\hat{\zeta}^{\left(\delta_{1}\right)}, \cdots, \hat{\zeta}^{\left(\delta_{r}\right)}, \hat{\zeta}^{\left(\omega_{0}\right)}, \cdots, \hat{\zeta}^{\left(\omega_{s}\right)}\right]^{\prime}$. A matriz $\Gamma$ é diag $\left[\Gamma_{\delta_{1}}, \Gamma_{\delta_{2}}\right.$, $\left.\cdots, \Gamma_{\delta_{r}}, \Gamma_{\omega_{0}}, \Gamma_{\omega_{2}}, \cdots, \Gamma_{\omega_{s}}\right]$, e cada bloco é dado por (3.18).

Cada coeficiente da função de transferência é encontrado por meio da transformada inversa de ondaletas, $\hat{\boldsymbol{\delta}}_{i}=\Lambda_{i}^{\delta \prime} \boldsymbol{\beta}$ e $\hat{\boldsymbol{\omega}}_{j}=\Lambda_{j}^{\omega \prime} \boldsymbol{\beta}$.

A matriz $\Lambda_{i}^{\delta}$ é uma matriz de $r+s+1$ blocos, cada bloco de dimensão $2^{j^{*}} \times T$. Somente o bloco $i$ é diferente de zero $i=1,2, \cdots, r$, e este é igual a $\mathbf{W}$, matriz de transformada inversa de ondaletas até o nível $j^{*}$.

Analogamente, a matriz $\Lambda_{i}^{\omega}$ é uma matriz de $r+s+1$ blocos, cada bloco de dimensão $2^{j^{*}} \times T$. Somente o bloco $j$ é diferente de zero $j=0,1, \cdots, s$, e este é igual a $\mathbf{W}$.

A verificação das propriedades estatísticas é feita como no caso mais simples. O desenvolvimento teórico é baseado em um único nível $j^{*}$, encontrando os coeficientes empíricos $\hat{\zeta}^{(i)}, i=\delta_{1}, \cdots, \delta_{r}, \omega_{0}, \cdots, \omega_{s}$. Os coeficientes $\hat{\boldsymbol{\beta}}^{(i)}$ são obtidos 
através de combinações lineares dos $\hat{\zeta}^{(i)}$. $\Gamma$ é matriz bloco diagonal que efetua essa transformação linear e tem dimensão $(r+s+1) 2^{j^{*}} \times(r+s+1) 2^{j^{*}}$.

\section{Proposição 3.2b:}

Sob as suposições $(1)-(6)$ temos que: $\boldsymbol{E}\left(\hat{\beta}_{j k}^{(i)}-\beta_{j k}^{(i)}\right)^{2}=O\left(T^{-1}\right)$, para todo $k$, $j<j^{*}$ e $i=\delta_{1}, \delta_{2}, \cdots, \delta_{r}$ e $\omega_{0}, \omega_{1}, \cdots, \omega_{s}$.

Proposição 3.3b: Sob as suposições (1) $-(6)$ temos que: $\boldsymbol{P}\left(\left(\hat{\beta}_{j k}^{(i)}-\beta_{j k}^{(i)}\right) / \sigma_{i j k} \geq x\right)=$ $(1-\Phi(x))(1+o(1))+O\left(T^{-\lambda}\right)$, para $i=\delta_{1}, \delta_{2}, \cdots, \delta_{r}, \omega_{0}, \omega_{1}, \cdots, \omega_{s}$.

As provas das Proposições $3.2 \mathrm{~b}$ e $3.3 \mathrm{~b}$ são feitas de maneira análoga as das Proposições 3.2 e 3.3. O uso dos Lemas $A .2$ e $A .3$ é imediato uma vez que os lemas valem para quaisquer $r$ es.

\subsubsection{Estimadores do Segundo Estágio}

No segundo estágio, a regressão é $Y_{t, T}$ sobre $\hat{Y}_{t-1, T}, \cdots, \hat{Y}_{t-r, T}$ e $X_{t-b, T}, X_{t-b-1, T}$, $\cdots, X_{t-b-s, T}$. A equação na forma matricial será:

$$
\mathbf{Y}=\left[\begin{array}{llllllll}
\mathbf{F}_{\hat{Y}_{(-1)}} & \mathbf{F}_{\hat{Y}_{(-2)}} & \cdots & \mathbf{F}_{\hat{Y}_{(-r)}} & \mathbf{F}_{X_{(-b)}} & \mathbf{F}_{X_{(-b-1)}} & \cdots & \mathbf{F}_{X_{(-b-s)}}
\end{array}\right]\left[\begin{array}{c}
\boldsymbol{\beta}^{\left(\delta_{1}\right)} \\
\vdots \\
\boldsymbol{\beta}^{\left(\delta_{r}\right)} \\
\boldsymbol{\beta}^{\left(\omega_{0}\right)} \\
\vdots \\
\boldsymbol{\beta}^{\left(\omega_{s}\right)}
\end{array}\right]+\mathbf{n},
$$

com

$$
\mathbf{n}=\left[\begin{array}{llll}
n_{t^{*}} & n_{t^{*}+1} & \cdots & n_{T}
\end{array}\right]^{\prime}
$$


em que $t^{*}=\max \{r, s+b\}$ e $\mathbf{n}$ é um processo médias móveis de ordem $2 r$. Para $0 \leq m \leq j^{*}$

$$
\mathrm{F}_{\hat{Y}_{(-m)}}=\left[\begin{array}{llll}
\boldsymbol{\Phi}_{\hat{Y}_{(-m)}} & \Psi_{\hat{Y}_{(-m)}}^{(0)} & \cdots & \Psi_{\hat{Y}_{(-m)}}^{\left(j^{*}-1\right)}
\end{array}\right] .
$$

O estimador do segundo estágio será:

$$
\tilde{\mathbf{B}}=\hat{\boldsymbol{\Xi}}^{-1} \hat{\Xi}_{x y}
$$

em que

$$
\begin{aligned}
& \tilde{\mathbf{B}}=\left[\begin{array}{llllll}
\tilde{\boldsymbol{\beta}}^{\left(\delta_{1}\right)} & \ldots & \tilde{\boldsymbol{\beta}}^{\left(\delta_{r}\right)} & \tilde{\boldsymbol{\beta}}^{\left(\omega_{0}\right)} & \ldots & \tilde{\boldsymbol{\beta}}^{\left(\omega_{s}\right)}
\end{array}\right]^{\prime}
\end{aligned}
$$

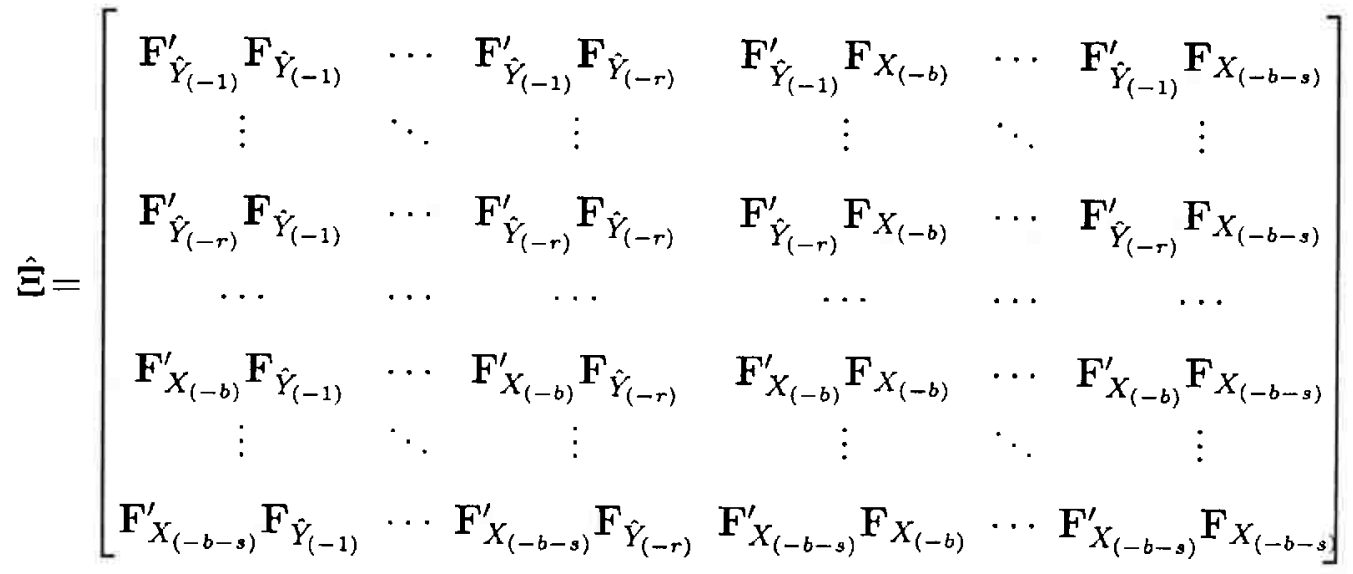

e

$$
\hat{\Xi}_{x y}=\left[\begin{array}{cc}
\mathbf{F}_{\hat{Y}_{(-1)}}^{\prime} \mathbf{Y} \\
\mathbf{F}_{\hat{Y}_{(-2)}}^{\prime} \mathbf{Y} \\
\vdots \\
\mathbf{F}_{\hat{Y}_{(-r)}}^{\prime} \mathbf{Y} \\
\ldots \\
\mathbf{F}_{X_{(-b)}}^{\prime} \mathbf{Y} \\
\mathbf{F}_{X_{(-b-1)}}^{\prime} \mathbf{Y} \\
\vdots \\
\mathbf{F}_{X_{(-b-s)}}^{\prime} \mathbf{Y}
\end{array}\right]
$$




\section{Capítulo 4}

\section{Simulações e Exemplos}

\subsection{Simulações}

Para verificar o desempenho dos estimadores propostos, fizemos simulaçōes usando o modelo de função de transferência (2.5), a saber:

$$
Y_{t, T}=\frac{\boldsymbol{\omega}_{t}(B)}{\boldsymbol{\delta}_{t}(B)} B^{b} X_{t, T}+\epsilon_{t, T},
$$

em que

$$
\begin{aligned}
\omega_{t}(B) & =\omega_{0}(t)+\omega_{1}(t) B+\cdots+\omega_{s}(t) B^{s} \\
\delta_{t}(B) & =1-\delta_{1}(t) B-\delta_{2}(t) B^{2}-\cdots-\delta_{r}(t) B^{r} .
\end{aligned}
$$

Apresentamos aqui os resultados de duas simulações: caso $1, \operatorname{com} b=0, s=0$ e $r=1$; caso 2 , com $b=0, s=0$ e $r=2$.

A série de entrada foi gerada utilizando um modelo $\mathrm{AR}(2)$ com os seguintes 
coeficientes

$$
a_{1}(u)=\left\{\begin{aligned}
1,69, & 0<u \leq 0,6 \\
-0,3, & 0,6<u \leq 1
\end{aligned}\right.
$$

e

$$
a_{2}(u)=0,81, \quad 0<u \leq 1
$$

Assim, $X_{u, T}=a_{1}(u) X_{(t-1) / T}+a_{2}(u) X_{(t-2) / T}+\varsigma_{u, T}$, sendo $\varsigma_{u, T}$ variáveis aleatórias normais independentes com $\boldsymbol{E}\left(\varsigma_{u, T}\right)=0, \operatorname{Var}\left(\varsigma_{u, T}\right)=1$, para $t=1,2, \cdots, T$ e $u=t / T$.

Usamos o limiar universal duro e como estimador da medida de variabilidade, $\hat{\sigma}$, o MAD ("median absolut deviance") para a escala mais fina $j^{*}-1$,

$$
\hat{\sigma}=\operatorname{mediana}\left\{\left|\hat{\beta}_{j^{*}-1, k}\right|: 0 \leq k \leq 2^{j^{*}-1}\right\} / 0,6745 \text {. }
$$

O intervalo bootstrap não paramétrico, $\operatorname{com} \gamma=0,95$, foi calculado após a aplicação do limiar. Para a construção do intervalo bootstrap foram utilizadas $\mathbb{B}=200$ réplicas

Além disso, fizemos $N=1000$ repetições de cada caso e construímos histogramas para diversos pontos ao longo das funções estimadas, apresentados no Apêndice B.

\subsection{Caso 1}

O modelo de função de transferência é

$$
Y_{t, T}=\frac{\omega_{0}\left(\frac{t}{T}\right)}{1-\delta_{1}\left(\frac{t}{T}\right) B} X_{t, T}+\epsilon_{t, T}, \quad t=1,2, \cdots, T, \quad u=t / T
$$


As funções escolhidas foram

$$
\begin{gathered}
\omega_{0}(u)=\left\{\begin{aligned}
2, & 0<u \leq 0,5 \\
-2, & 0,5<u \leq 1
\end{aligned}\right. \\
\delta_{1}(u)=\left\{\begin{array}{cc}
0,6, & 0<u \leq 0,25 \\
-0,5, & 0,25<u \leq 0,75 \\
0,6, & 0,75<u \leq 1
\end{array}\right.
\end{gathered}
$$

Foi usada a ondaleta de Haar. As séries de entrada e saída estão na Figura 4.1. As estimativas para os coeficientes estão nas Figuras 4.2 e 4.3.

\section{Série de Entrada}

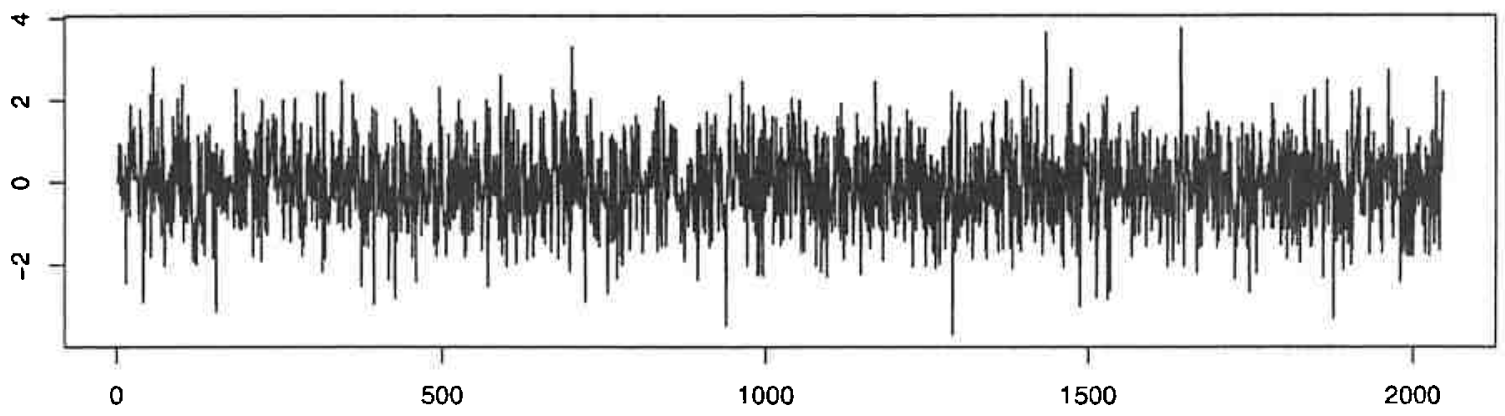

(a)

Série de Saida

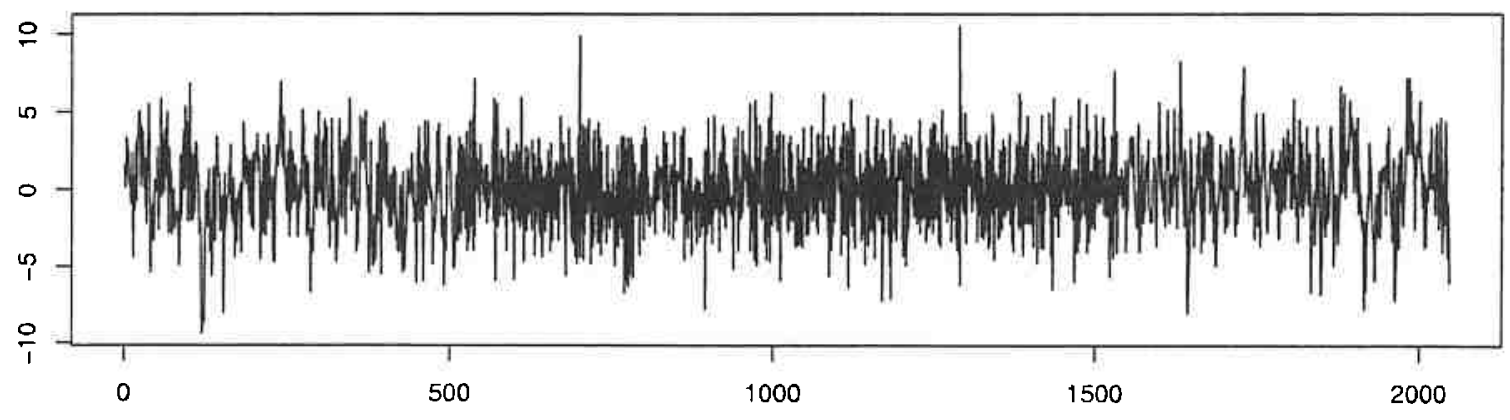

(b)

Figura 4.1: Séries Simuladas de Entrada (a) e Saída (b) do Caso 1. 


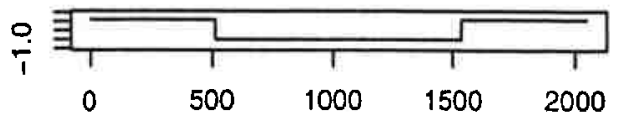

(a)

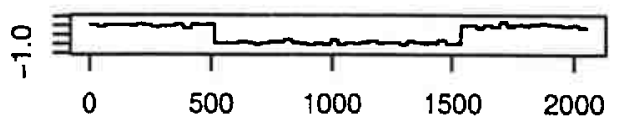

(c)

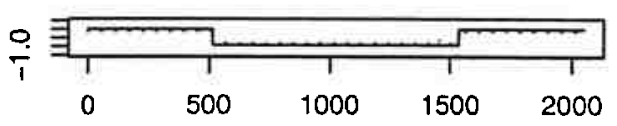

(e)

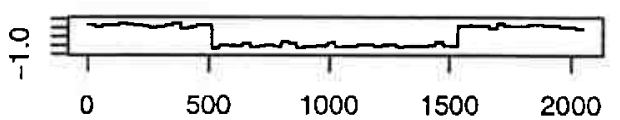

(g)

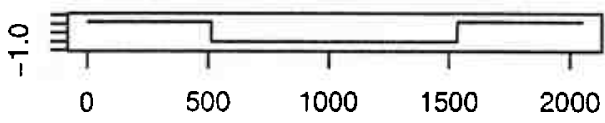

(i)

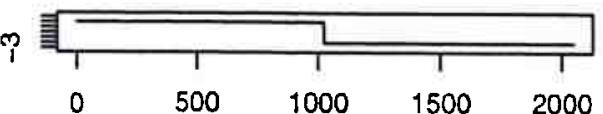

(b)

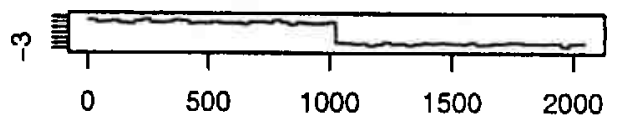

(d)

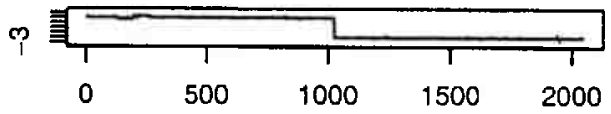

(f)

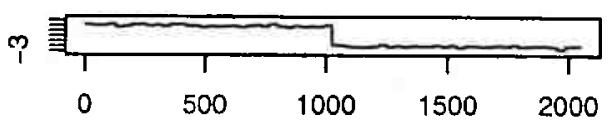

(h)

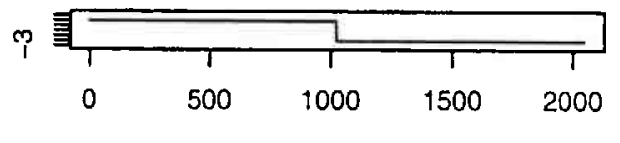

(j)

Figura 4.2: Resultados da Simulação para o Caso 1. (a) e (b) são $\delta_{1}(t)$ e $\omega_{0}(t)$, (c) e (d) $\hat{\delta}_{1}(t)$ e $\hat{\omega}_{0}(t)$, (e) e (f) $\hat{\delta}_{1}^{(L)}(t)$ e $\hat{\omega}_{0}^{(L)}(t)$ (linha cheia) e intervalo bootstrap de $95 \%$ (linha pontilhada), (g) e $(\mathrm{h}) \tilde{\delta}_{1}(t)$ e $\tilde{\omega}_{0}(t)$ e; (i) e $(\mathrm{j}) \tilde{\delta}_{1}(t)^{(L)}(t)$ e $\tilde{\omega}_{0}^{(L)}(t)$ (linha cheia) e intervalo bootstrap de $95 \%$ (linha pontilhada). 


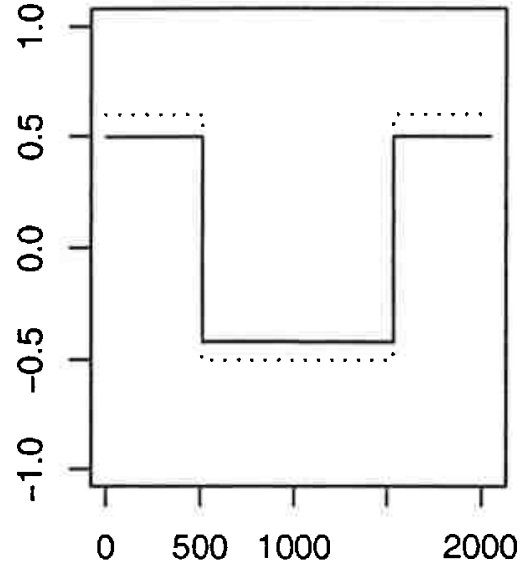

(a)

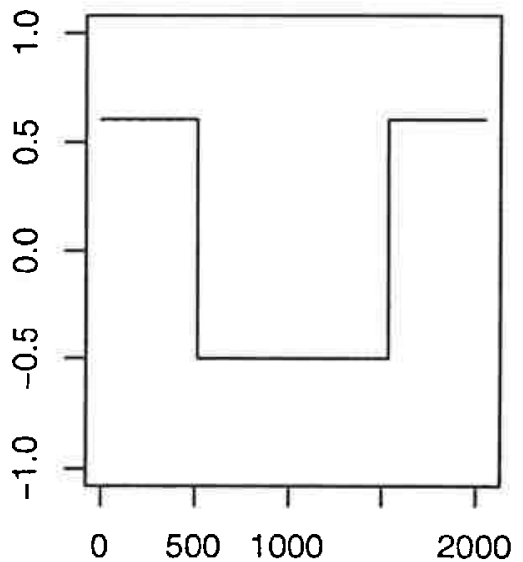

(c)

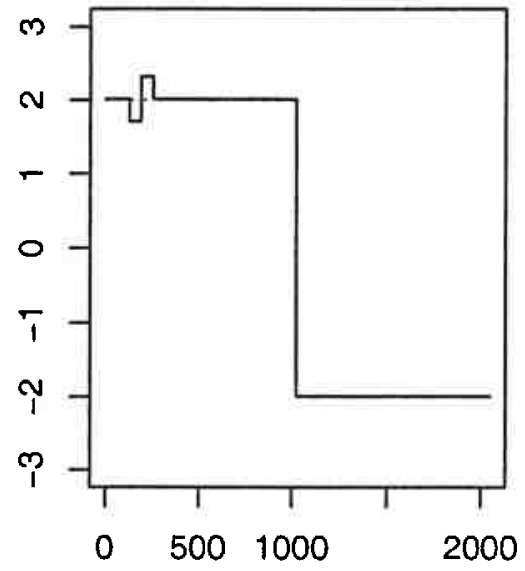

(b)

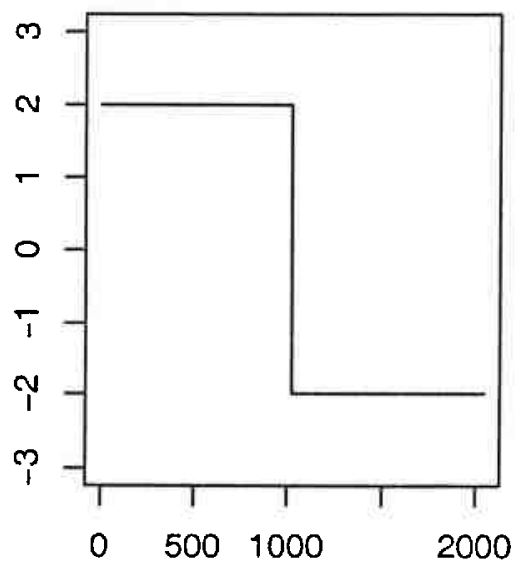

(d)

Figura 4.3: Resultados da Simulação, primeiro e segundo estágios, para o Caso 1. (a) e (b) as estimativas $\hat{\delta}_{1}^{(L)}(t)$ e $\hat{\omega}_{0}^{(L)}(t)$ (linha cheia) e; (c) e (d) as estimativas $\tilde{\delta}_{1}^{(L)}(t)$ e $\tilde{\omega}_{0}^{(L)}(t)$ (linha cheia). 
Na Figura 4.2 temos as funções verdadeiras $\delta_{1}(t)$ e $\omega_{0}(t)$ em (a) e (b), as estimativas $\hat{\delta}_{1}(t)$ e $\hat{\omega}_{0}(t)(\mathrm{c})$ e $(\mathrm{d})$, as estimativas $\hat{\delta}_{1}^{(L)}(t)$ e $\hat{\omega}_{0}^{(L)}(t)$ (linha cheia) e intervalo bootstrap de $95 \%$ (linha pontilhada) em (e) e (f), as estimativas $\tilde{\delta}_{1}(t)$ e $\tilde{\omega}_{0}(t)$ em (g) e (h) e, as estimativas $\tilde{\delta}_{\mathbf{1}}^{(L)}(t)$ e $\tilde{\omega}_{0}^{(L)}(t)$ (linha cheia) e intervalo bootstrap de 95\% (linha pontilhada) em (i) e (j).

Na Figura 4.3 temos as estimativas $\hat{\delta}_{1}^{(L)}(t)$ e $\hat{\omega}_{0}^{(L)}(t)$ (linha cheia) em (a) e (b); as estimativas $\tilde{\delta}_{1}^{(L)}(t)$ e $\tilde{\omega}_{0}^{(L)}(t)$ (linha cheia) em (c) e (d), as linhas pontilhadas indicam o valor verdadeiro da função.

Na coluna da direita das Figura 4.2 e 4.3 temos as estimativas para o coeficiente $\delta_{1}(t)$ e nas colunas da esquerda, as estimativas para o coeficiente $\omega_{0}(t)$. Observamos que as estimativas estão próximas da forma simulada. Ao analisarmos a Figura 4.3 vemos que as estimativas para $\omega_{0}(t)$ são coincidentes com a função simulada. Para as estimativas de $\delta_{1}(t)$ há diferença entre os dois estágios. No primeiro estágio, a função estimada é viesada e, no segundo estágio, a estimativa é corrigida, diminuindo o viés. A correção aqui mencionada é verificada na análise dos histogramas apresentados no Apêndice B, Figuras B.1 a B.4 para $\delta_{1}(t)$ e Figuras B.5 a B.8 para $\omega_{0}(t)$. A análise desse histogramas nos mostra que as curvas $\hat{\delta}_{1}(t), \hat{\omega}_{0}(t), \tilde{\delta}_{1}(t)$ e $\tilde{\omega}_{0}(t)$ apresentam distribuição aparentemente normal, para os valores de $t$ anotados; já para as curvas $\hat{\delta}_{1}^{(L)}(t), \hat{\omega}_{0}^{(L)}(t), \tilde{\delta}_{1}^{(L)}(t)$ e $\tilde{\omega}_{0}^{(L)}(t)$ as distribuições são mais concentradas.

Os valores de $\hat{\omega}(t)$ e $\tilde{\omega}(t)$ são muito próximos.

Analisando os histogramas referentes as estimativas da função $\delta(t)$ vemos que existe uma diferença entre as estimativas do primeiro e do segundo estágio. A estimativa do segundo estágio está corrigida para o viés. 


\subsection{Caso 2}

O modelo de função de transferência é,

$$
Y_{u, T}=\frac{\omega_{0}(u)}{1-\delta_{1}(u) B-\delta_{2}(u) B^{2}} X_{u, T}+\epsilon_{u, T}, \quad u \in(0,1]
$$

As funções escolhidas para esse caso foram:

$$
\begin{array}{rlrl}
\omega_{0}(u)=9 \cos (2 \pi u+\pi), & u \in(0,1], \\
\delta_{1}(u)=0,5 \cos (3 \sin (\pi u)), & & u \in(0,1], \\
\delta_{2}(u)=0,5 \sin (\cos (2 \pi u)), & & u \in(0,1] .
\end{array}
$$

A ondaleta usada foi a $L A 8$.

A série de entrada é a mesma do caso 1 ou seja, $X_{u, T}=a_{1}(u) X_{(t-1) / T}+$ $a_{2}(u) X_{(t-2) / T}+\varsigma_{u, T}$, sendo $\varsigma_{u, T}$ são variáveis aleatórias normais independentes com $\boldsymbol{E}\left(\varsigma_{u, T}\right)=0$ e $\operatorname{Var}\left(\varsigma_{u, T}\right)=1$ para $t=1,2, \cdots, T, u=t / T, \mathrm{e}$

$$
a_{1}(u)= \begin{cases}1,69, & 0<u \leq 0,6 \\ -0,3, & 0,6<u \leq 1\end{cases}
$$

e

$$
a_{2}(u)=0,81 \quad 0<u \leq 1
$$

As séries de entrada e saída estão na Figura 4.4. As estimativas para os coeficientes estão nas Figuras 4.5 e 4.6. 
Série de Entrada

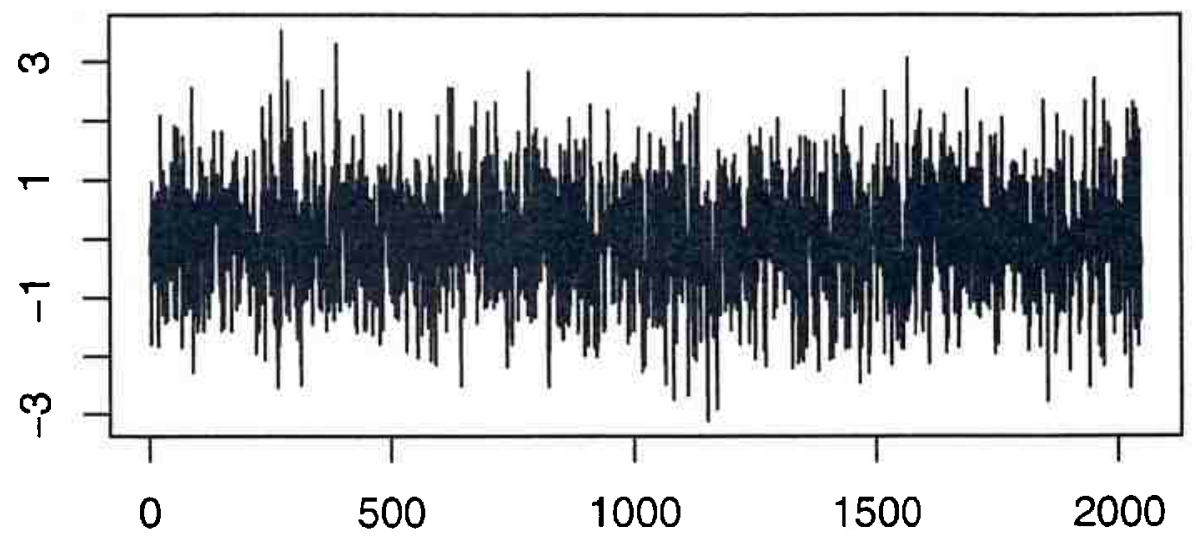

(a)

Série de Saída

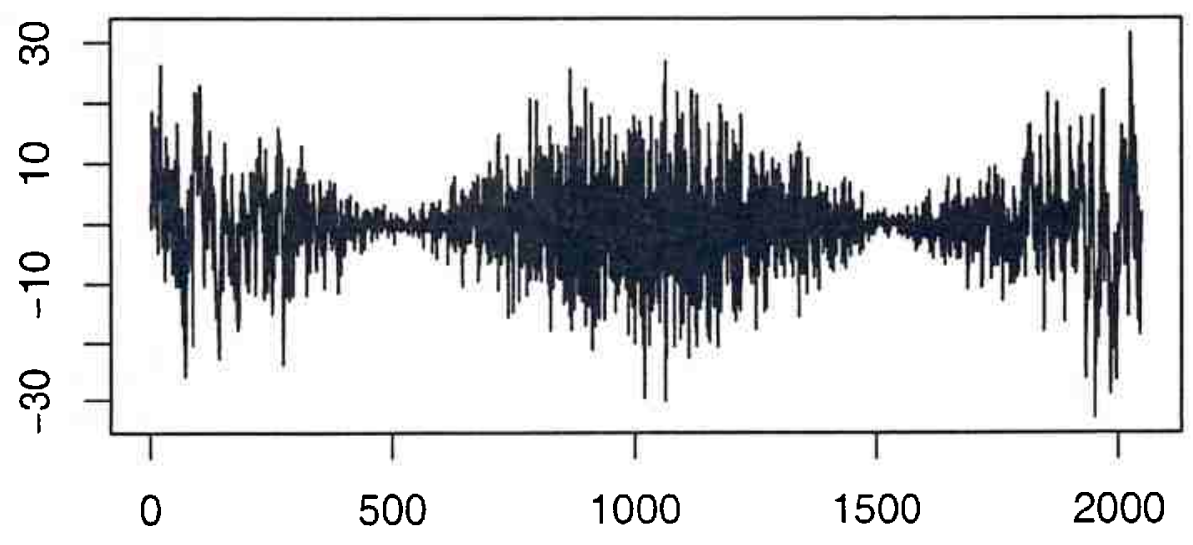

(b)

Figura 4.4: Séries Simuladas de Entrada (a) e Saída (b) do Caso 2. 


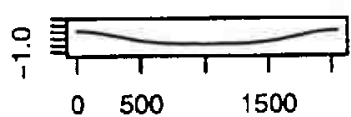

(a)

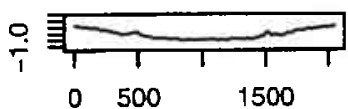

(d)

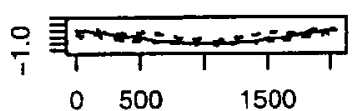

(g)

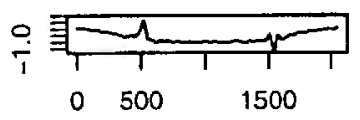

(j)

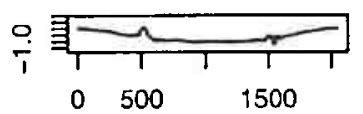

(m)

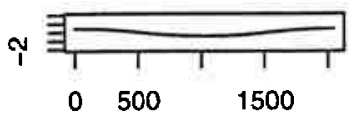

(b)

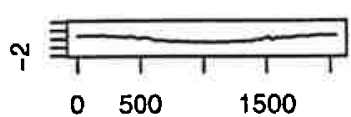

(e)

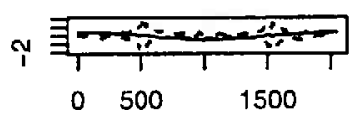

(h)

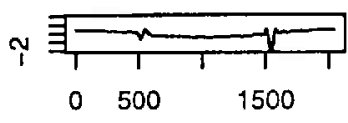

(k)

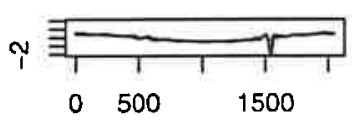

(n)

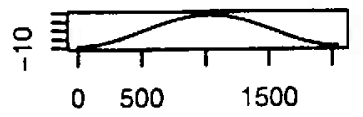

(c)

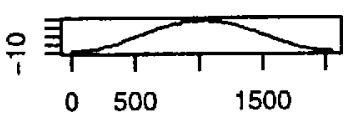

(1)

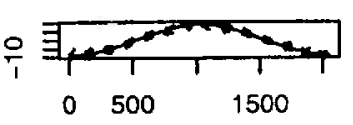

(i)

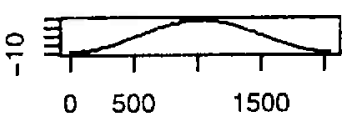

(I)

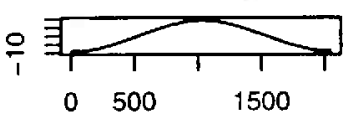

(o)

Figura 4.5: Resultados da Simulação para o Caso 2. (a), (b) e (c) são $\delta_{1}(t)$, $\delta_{2}(t)$ e $\omega_{0}(t) ;(\mathrm{d}),(\mathrm{e})$ e (f) $\hat{\delta}_{1}(t), \hat{\delta}_{2}(t)$ e $\hat{\omega}_{0}(t) ;(\mathrm{g}),(\mathrm{h})$ e $(\mathrm{i}) \hat{\delta}_{1}^{(L)}(t), \hat{\delta}_{2}^{(L)}(t)$ e $\hat{\omega}_{0}^{(L)}(t)$ (linha cheia) e intervalo bootstrap de $95 \%$ (linha pontilhada); (j), (k) e (l) $\tilde{\delta}_{1}(t)$, $\tilde{\delta}_{2}(t)$ e $\tilde{\omega}_{0}(t)$ e; $(\mathrm{m}),(\mathrm{n})$ e $(\mathrm{o}) \hat{\delta}_{1}^{(L)}(t), \hat{\delta}_{2}^{(L)}(t)$ e $\hat{\omega}_{0}^{(L)}(t)$ (linha cheia) e intervalo bootstrap de $95 \%$ (linha pontilhada). 


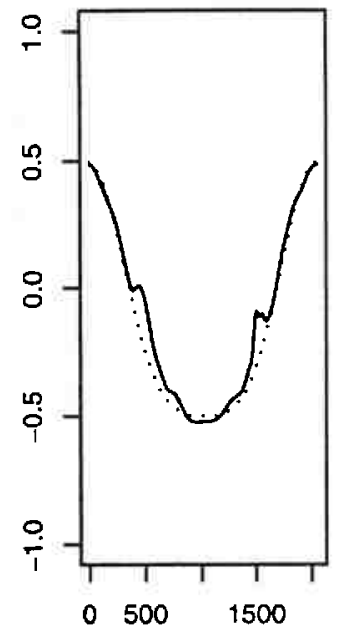

(a)

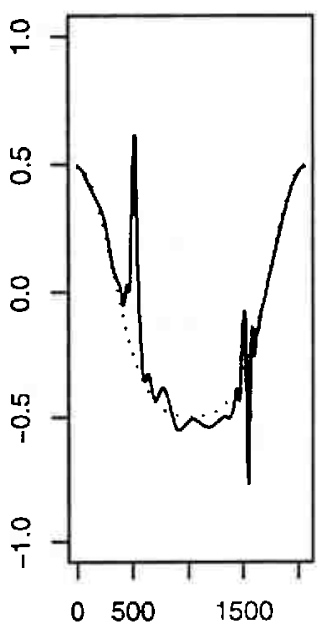

(d)

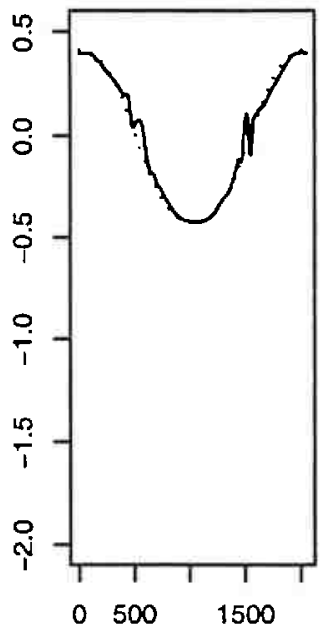

(b)

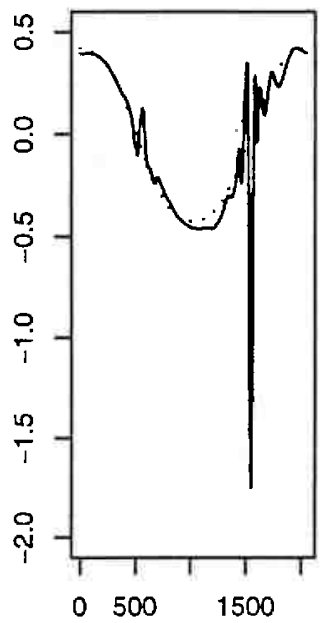

(e)

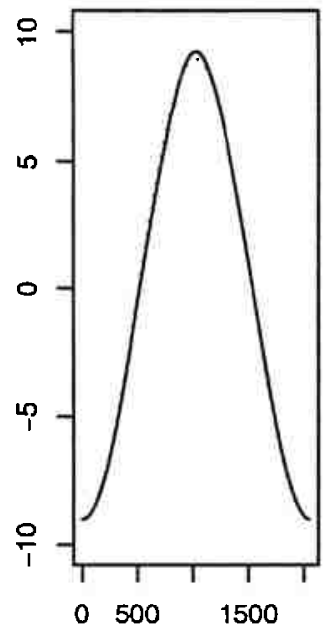

(c)

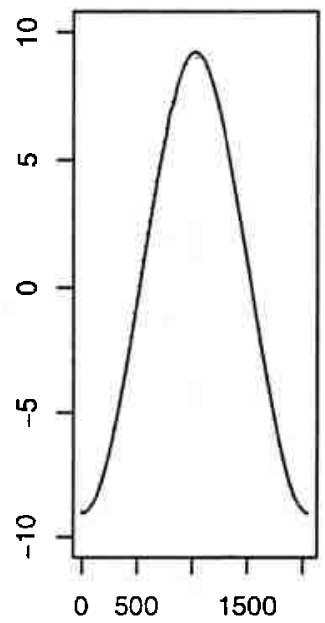

(f)

Figura 4.6: Resultados da Simulação, nos dois estágios, para o Caso 2. (a), (b) e (c) são as estimativas $\hat{\delta}_{1}^{(L)}(t), \hat{\delta}_{2}^{(L)}(t)$ e $\hat{\omega}_{0}^{(L)}(t)$ (linha cheia); e (d), (e) e (f) as estimativas $\tilde{\tilde{\delta}}_{1}^{(L)}(t), \tilde{\delta}_{2}^{(L)}(t)$ e $\tilde{\omega}_{0}^{(L)}(t)$ (linha cheia). 
Na Figura 4.5 temos as funções verdadeiras $\delta_{1}(t), \delta_{2}(t)$ e $\omega_{0}(t)$ em (a), (b) e (c), as estimativas $\hat{\delta}_{1}(t), \hat{\delta}_{2}(t)$ e $\hat{\omega}_{0}(t)$ em $(\mathrm{d}),(\mathrm{e})$ e $(\mathrm{f})$, as estimativas $\hat{\delta}_{1}^{(L)}(t), \hat{\delta}_{2}^{(L)}(t)$ e $\hat{\omega}_{0}^{(L)}(t)$ (linha cheia) e intervalo bootstrap de $95 \%$ (linha pontilhada) em (g), (h) e (i), as estimativas $\tilde{\delta}_{1}(t), \tilde{\delta}_{2}(t)$ e $\tilde{\omega}_{0}(t)$ em $(j),(\mathrm{k})$ e $(\mathrm{l})$ e, as estimativas $\hat{\delta}_{1}^{(L)}(t)$, $\hat{\delta}_{2}^{(L)}(t)$ e $\hat{\omega}_{0}^{(L)}(t)$ (linha cheia) e intervalo bootstrap de $95 \%$ (linha pontilhada) em $(\mathrm{m}),(\mathrm{n})$ e $(\mathrm{o})$.

A Figura 4.6 contém as estimativas $\hat{\delta}_{1}^{(L)}(t), \hat{\delta}_{2}^{(L)}(t)$ e $\hat{\omega}_{0}^{(L)}(t)$ (linha cheia) em (a), (b) e (c), as e, as estimativas $\tilde{\delta}_{1}^{(L)}(t), \tilde{\delta}_{2}^{(L)}(t)$ e $\tilde{\omega}_{0}^{(L)}(t)$ (linha cheia) em (d), (e) e (f). As linhas pontilhadas indicam os valores verdadeiros das funções. Nas colunas da esquerda temos a função $\delta_{1}(t)$, nas colunas do meio a função $\delta_{2}(t)$ e nas colunas da direita a função $\omega_{0}(t)$. Observamos na Figura 4.5 que nas estimativas do primeiro estágio de $\delta_{1}(t)$ e $\delta_{2}(t)$ há perturbações em duas regiões. No segundo estágio o intervalo de tempo, que a função apresenta problema, é diminuido, embora o problema esteja mais acentuado. Devemos destacar aqui, que as regiões em que as funções $\delta_{1}(t)$ e $\delta_{2}(t)$ apresentaram grande variabilidade da estimativa é exatamente ao redor dos pontos em que a função $\omega_{0}(t)$ assume o valor zero. Esse problema é explicado pelo fato de estarmos estimando uma função racional. Teremos infinitas possibilidades não nulas para a função do denominador nos pontos em que a função do numerador assumir o valor zero.

Na Figura 4.6 podemos comparar as estimativas de $\hat{\delta}_{1}^{(L)}(t), \hat{\delta}_{2}^{(L)}(t)$ e $\hat{\omega}_{0}^{(L)}(t)$ com as estimativas de $\tilde{\delta}_{1}^{(L)}(t), \tilde{\delta}_{2}^{(L)}(t)$ e $\tilde{\omega}_{0}^{(L)}(t)$. Observamos que as estimativas para $\omega_{0}(t)$ são muito próximas entre si e também do valor verdadeiro. Já para as estimativas de $\delta_{1}(t)$ e $\delta_{2}(t)$, no primeiro estágio, temos uma superestimaçāo das funções ao redor de $t=500$ e $t=1500$. No segundo estágio, ao redor de $t=500$ para a estimativa de $\delta_{1}(t)$ e ao redor de $t=1500$ para a estimativa de $\delta_{2}(t)$ as oscilações apresentam maior amplitude, atingindo, porém, menores intervalos de $t$. Novamente devemos destacar que a função $\omega_{0}(t)$ assume o valor zero em $t=512 \mathrm{e}$ 
$t=1536$.

No Apêndice B, nas Figuras B.9 a B.12 apresentamos as distribuições dos diversos estágios de $\delta_{1}(t)$, Figuras B.13 a B.16 as distribuições dos diversos estágios de $\delta_{2}(t)$ e, Figuras B.17 a B.20 as distribuições dos diversos estágios de $\omega_{0}(t)$.

A análise desses histogramas nos mostra que as curvas $\hat{\delta}_{1}(t), \hat{\delta}_{2}(t), \hat{\omega}_{0}(t), \tilde{\delta}_{1}(t)$, $\tilde{\delta}_{2}(t)$ e $\tilde{\omega}_{0}(t)$ apresentam distribuição aparentemente normal, para os valores de $t$ anotados; já para as curvas $\hat{\delta}_{1}^{(L)}(t), \hat{\delta}_{2}^{(L)}(t), \hat{\omega}_{0}^{(L)}(t), \tilde{\delta}_{1}^{(L)}(t), \tilde{\delta}_{2}^{(L)}(t)$ e $\tilde{\omega}_{0}^{(L)}(t)$ as distribuições são mais concentradas, leptocúrticas. Os valores de $\hat{\omega}(t)$ e $\tilde{\omega}(t)$ são muito próximos.

Para esse exemplo também notamos que a estimativa do segundo estágio está mais próxima do original.

\subsection{Exemplo Real}

Os dados se referem às séries diárias de chuva (em $\mathrm{mm}$ ), $X_{t, T}$ e de temperatura máxima $\left(\mathrm{em}^{\circ} \mathrm{C}\right), Y_{t, T}$, na cidade de Ribeirão Preto, SP, de 01 de janeiro de 1988 a 25 de dezembro de 1996. Houve quatro observações perdidas da série de chuva e seis da série da temperatura máxima; essas falhas foram substituídas por valores interpolados. As séries originais estão mostradas na Figura 4.7. Estas séries apresentam sazonalidade de aproximadamente 365 dias, como verificamos na Figura 4.8 , contendo os periodogramas das séries de entrada $\left(X_{t, T}\right)$ e saída $\left(Y_{t, T}\right)$. A estatística do teste de Fisher para a componente máxima do periodograma (nona componente, $f=1 / 365$ ) para a série da chuva é 0,0743 e para a série da temperatura máxima é 0,1617 , ambas com $P$-valor $<0,0001$. As séries sazonalmente ajustadas, $\tilde{X}_{t, T}=X_{t, T}-X_{t-365 . T}$ e $\tilde{Y}_{t . T}=Y_{t, T}-Y_{t-365 . T}$, estão na Figura 4.9 . 
Originalmente tínhamos 3283 observações de cada série. Depois do ajustamento da sazonalidade ficamos com 2917 observações.

Os periodogramas para as séries sazonalmente ajustadas estão na Figura 4.10. Não notamos picos significativos nestes periodogramas. Vamos trabalhar com as 2048 observações finais ajustadas pela média.

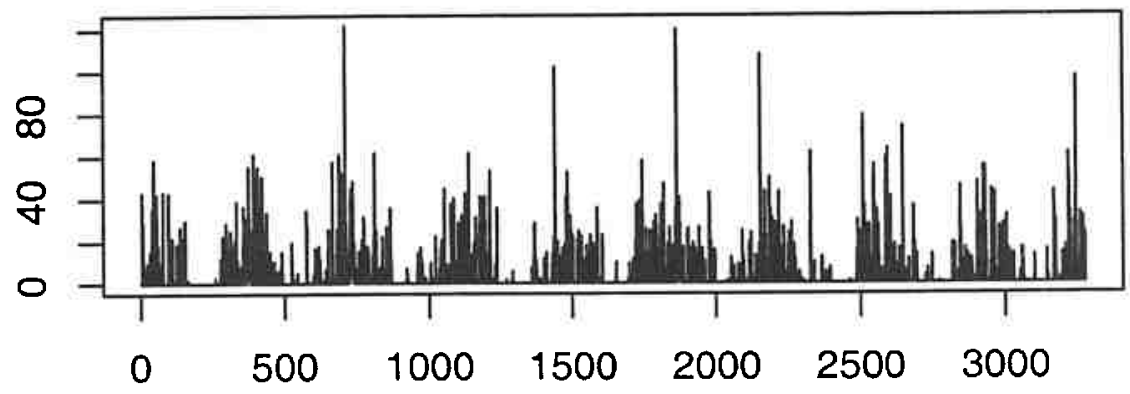

(a)

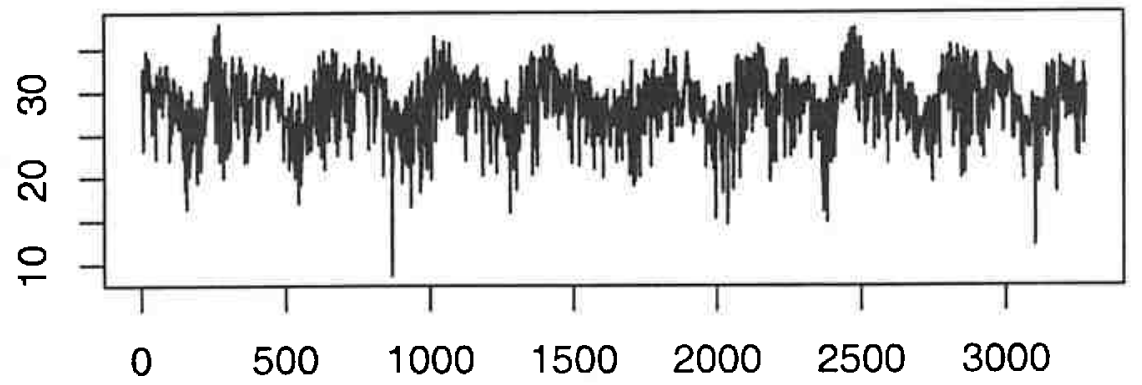

(b)

Figura 4.7: Séries Originais (a) Chuva $X_{t, T}$ e (b) Temperatura Máxima $Y_{t, T}$. 


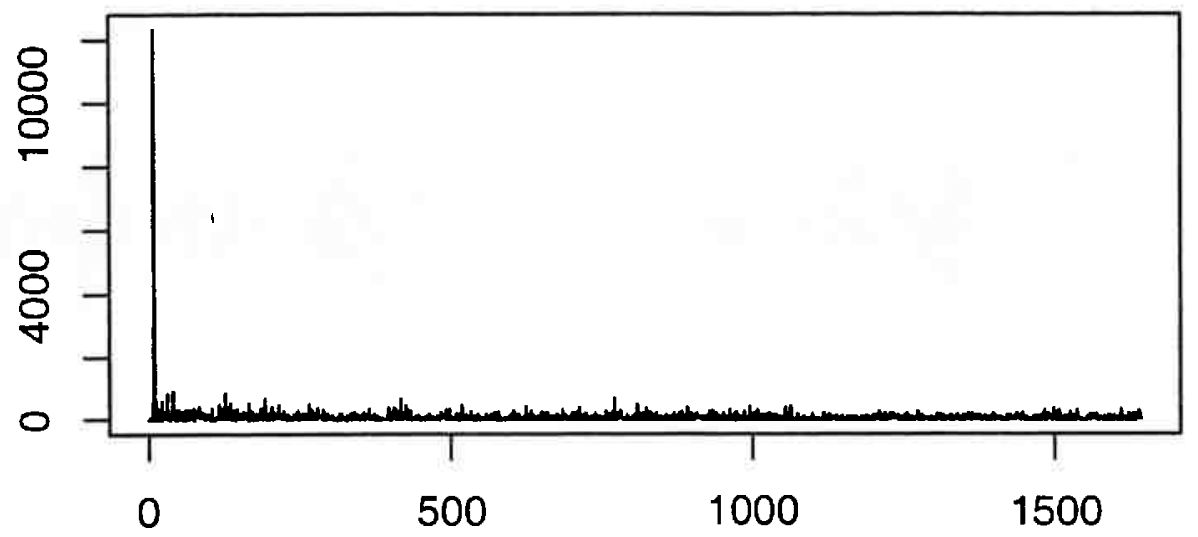

(a)

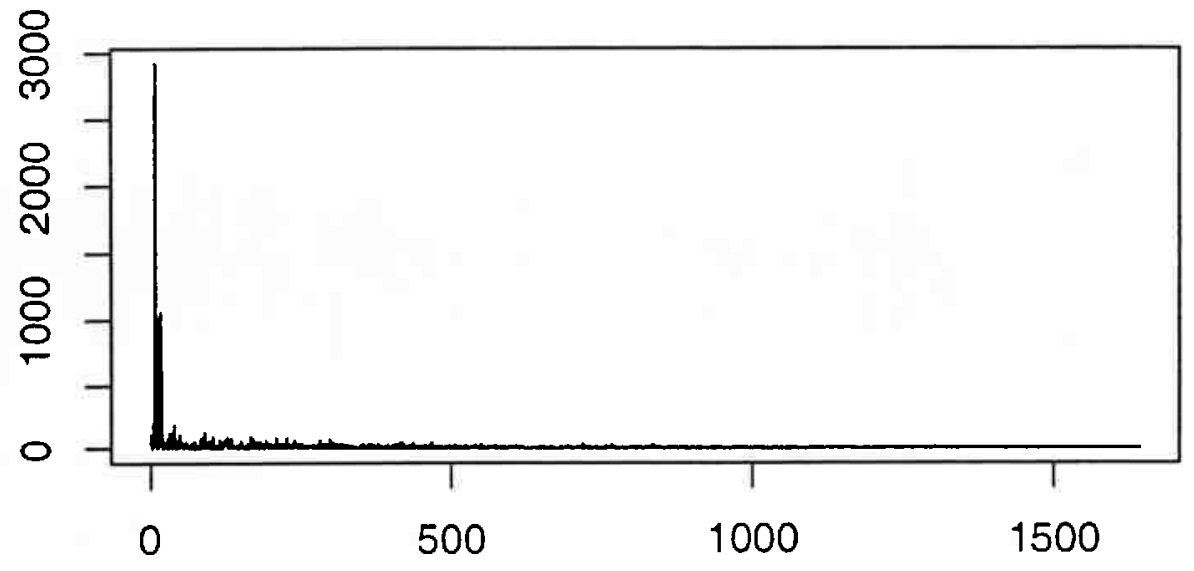

(b)

Figura 4.8: Periodogramas das Séries (a) de Entrada - Chuva e (b) Saída -Temperatura Náxima. 


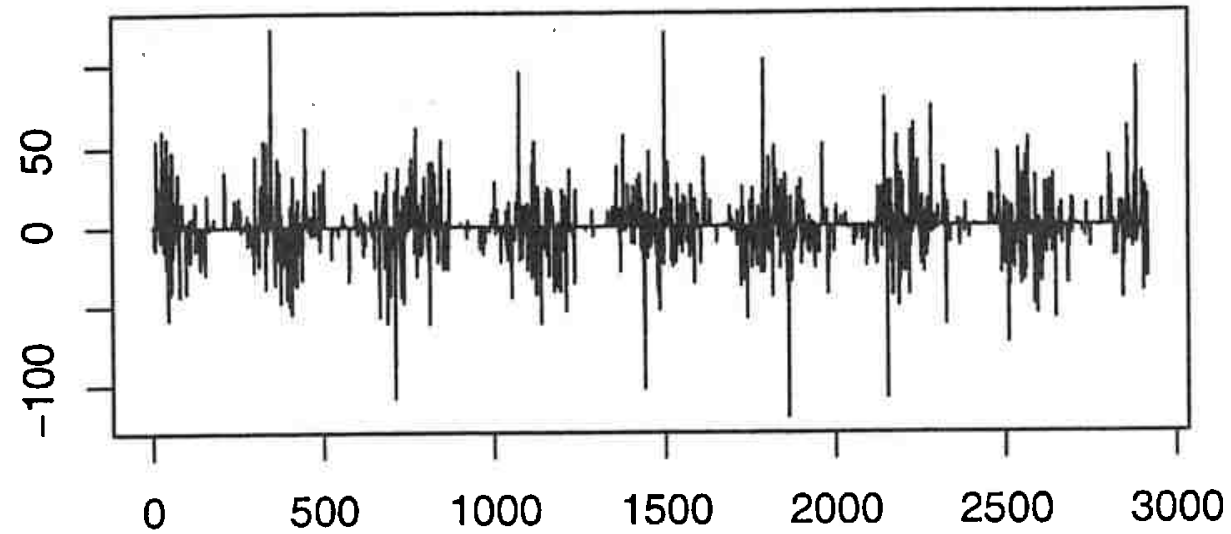

(a)

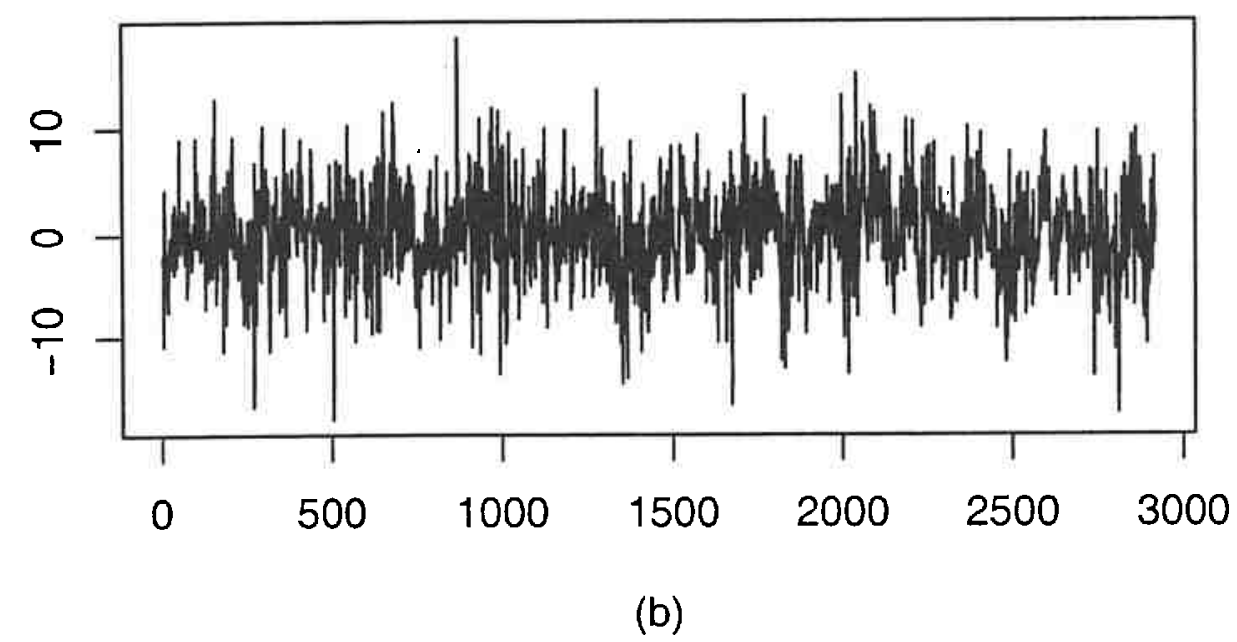

Figura 4.9: Séries Sazonalmente Ajustadas (a) Chuva $\tilde{X}_{t, T}=X_{t, T}-X_{t-365, T}$ e (b) Temperatura Máxima $\tilde{Y}_{t, T}=Y_{t, T}-Y_{t-365, T}$. 


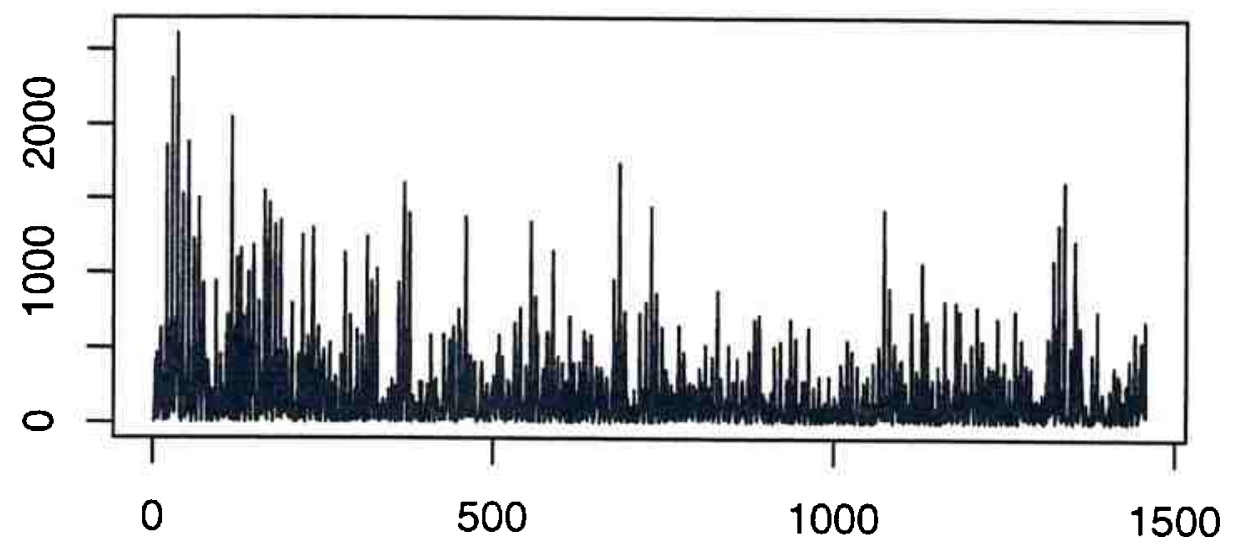

(a)

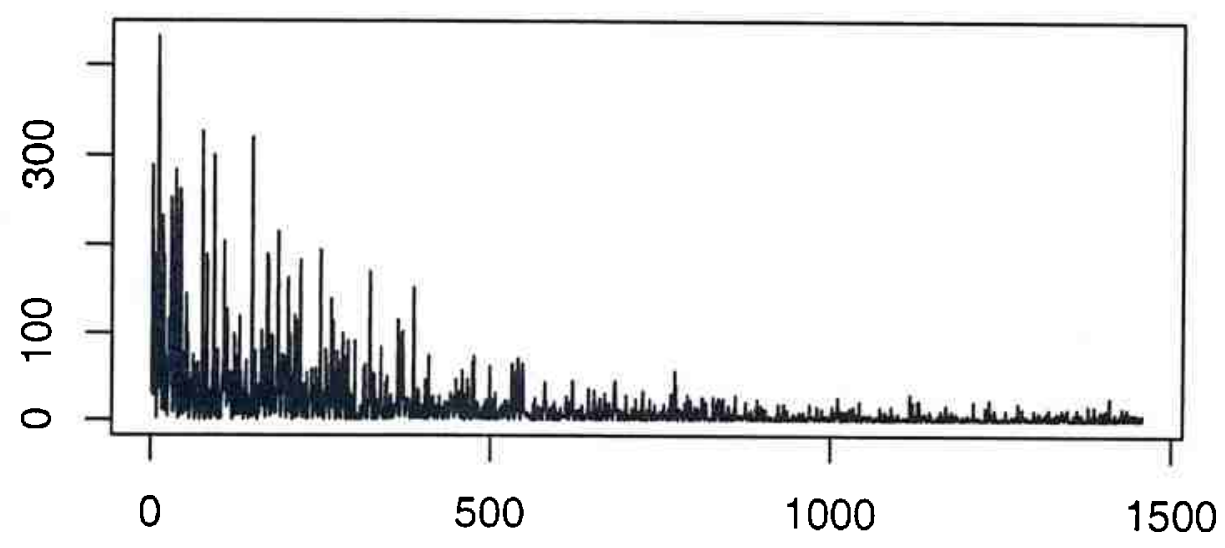

(b)

Figura 4.10: Periodogramas das Séries Sazonalmente Ajustadas de (a) Entrada $\tilde{X}_{t, T}$ e (b) Saída $\tilde{Y}_{t . T}$. 
Para escolhermos um modelo que se ajuste aos dados, testamos os três modelos a seguir:

$$
\begin{array}{ll}
\text { Modelo } \quad M_{1}: Y_{t, T}=\frac{\omega_{0}(t)}{1-\delta_{1}(t) B} X_{t, T}+\epsilon_{t, T} \\
\text { Modelo } \quad M_{2}: Y_{t, T}=\frac{\omega_{0}(t)+\omega_{1}(t) B}{1-\delta_{1}(t) B} X_{t, T}+\epsilon_{t, T} . \\
\text { Modelo } \quad M_{3}: \quad Y_{t, T}=\frac{\omega_{0}(t)}{1-\delta_{1}(t) B-\delta_{2}(t) B^{2}} X_{t, T}+\epsilon_{t, T} .
\end{array}
$$

As estimativas finais para os coeficientes dos Modelos $M_{1}, M_{2}$ e $M_{3}$ estão nas Figuras $4.11,4.12$, e 4.13 respectivamente.

Notamos que $\tilde{\delta}_{1}^{(L)}(t)$ em todos os casos assume valores positivos, ao redor de 0,4 . Na Figura 4.11 observamos, pelo intervalo bootstrap que há quatro regiões de maior variabilidade. Vemos na Figura 4.12 que, nessas regiões, o intervalo bootstrap é amplo. A função $\tilde{\omega}_{0}^{(L)}$ apresenta valores quase sempre negativos, com intervalos bootstrap estreitos e também notamos quatro regiões com picos mínimos. Os picos mínimos se localizam nos mesmos pontos que os picos observados nos intervalos de confiança para $\tilde{\delta}_{1}^{(L)}(t)$.

A função $\tilde{\omega}_{1}^{(L)}(t)$, na Figura 4.12, tem intervalo bootstrap que contém o valor zero para todos os valores de $t, t=1,2, \cdots, 2048$. Isto indica que o modelo ajustado está superparametrizado.

A função $\tilde{\delta}_{2}^{(L)}(t)$, na Figura 4.13 , também não apresenta valores significativamente diferentes de zero para todos os valores de $t, t=1,2, \cdots, 2048$. Novamente temos um modelo superparametrizado, levando-nos a concluir que, de maneira parcimoniosa, o Modelo 1 é o modelo que melhor se ajusta aos dados.

No Apêndice C estão as estimativas dos dois estágios, com e sem transformação por limiares para essas funções.

Os valores de $t$ para os quais a função $\tilde{\omega}_{0}^{(L)}$ atinge mínimos locais são: $t=38$, 
444, 1152 e 1543. Na Figura 4.17 temos as séries usadas no ajuste $\tilde{X}_{t, T}$ e $\tilde{Y}_{t, T}$ com a localização desses pontos. Observamos que estes pontos anotados nas Figuras 4.17 e 4.18 são os períodos de inverno nos quais temos estiagem prolongada, nessa situação a relação entre chuva e temperatura máxima se altera. 


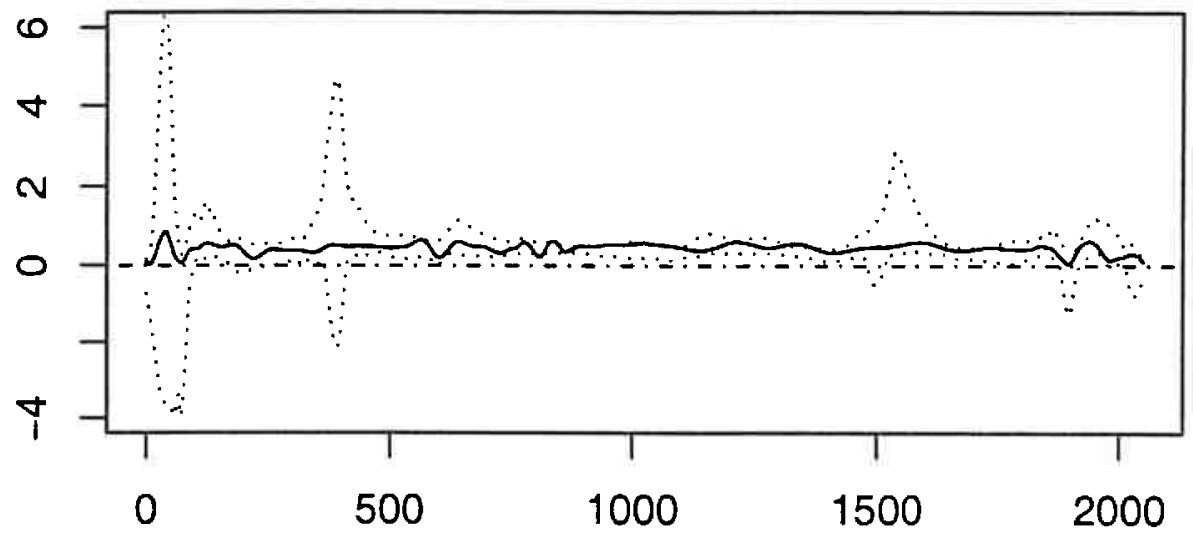

(a)

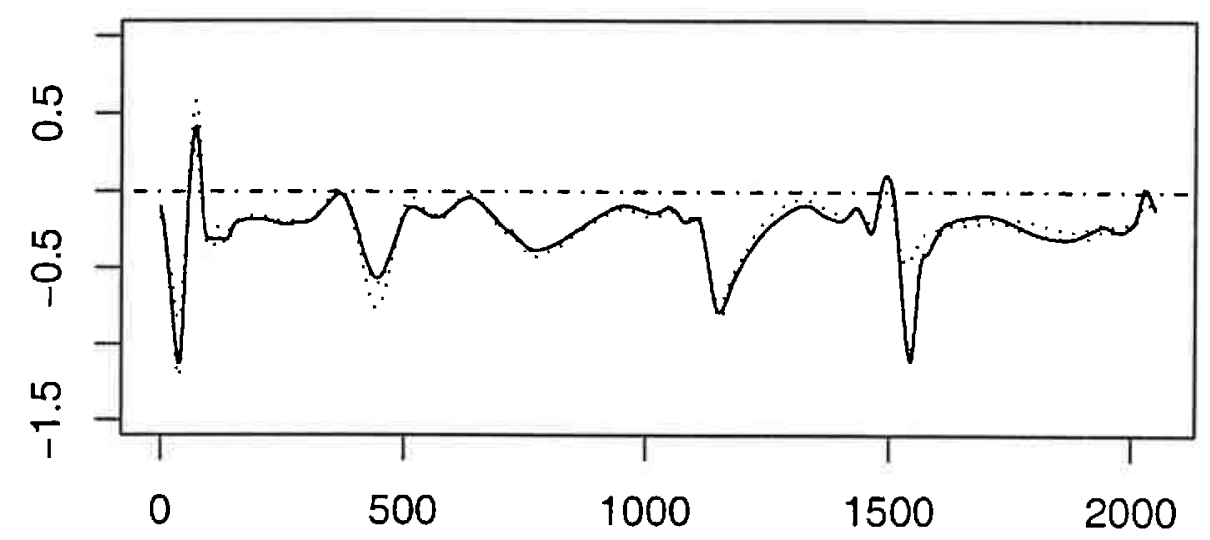

(b)

Figura 4.11: Resultados do ajuste do modelo 1, as Figuras (a) e (b) são as estimativas $\tilde{\delta}_{1}^{(L)}(t)$ e $\tilde{\omega}_{0}^{(L)}(t)$ (linha cheia) e intervalo bootstrap de $95 \%$ (linha pontilhada). 


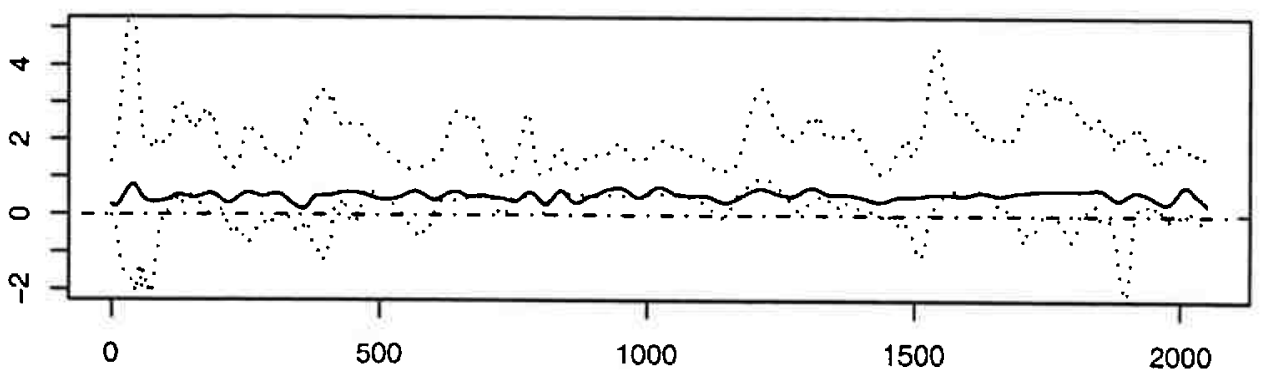

(a)

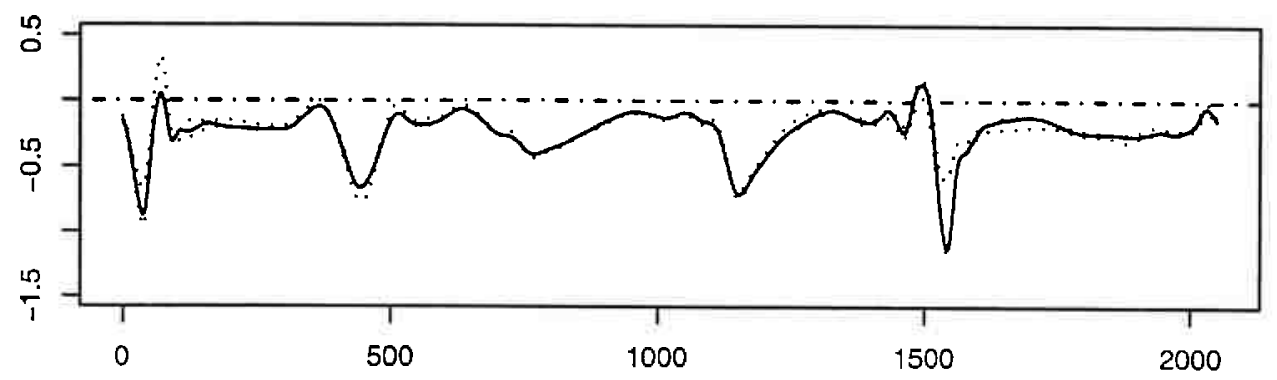

(b)

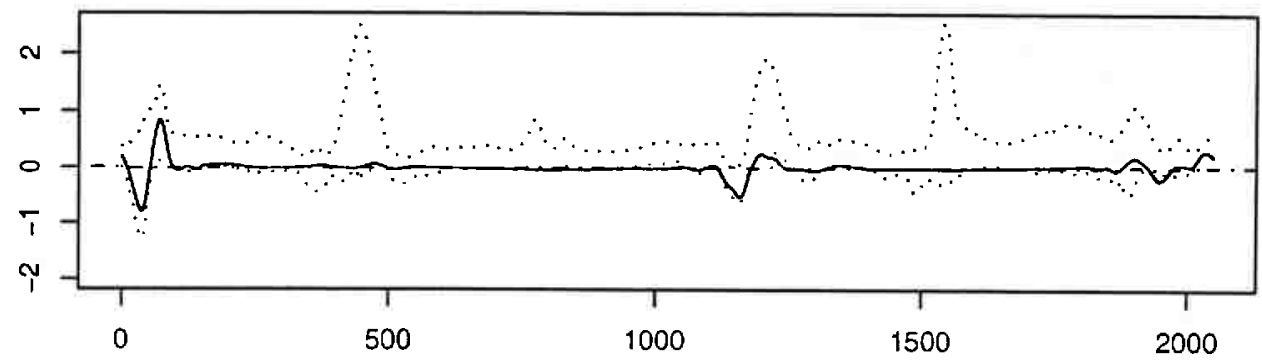

(c)

Figura 4.12: Resultados do ajuste do modelo 2, as Figuras (a), (b) e (c) são as estimativas $\tilde{\delta}_{1}^{(L)}(t), \tilde{\omega}_{0}^{(L)}(t)$ e $\tilde{\omega}_{1}^{(L)}(t)$ (linha cheia) e intervalo bootstrap de $95 \%$ (linha pontilhada). 


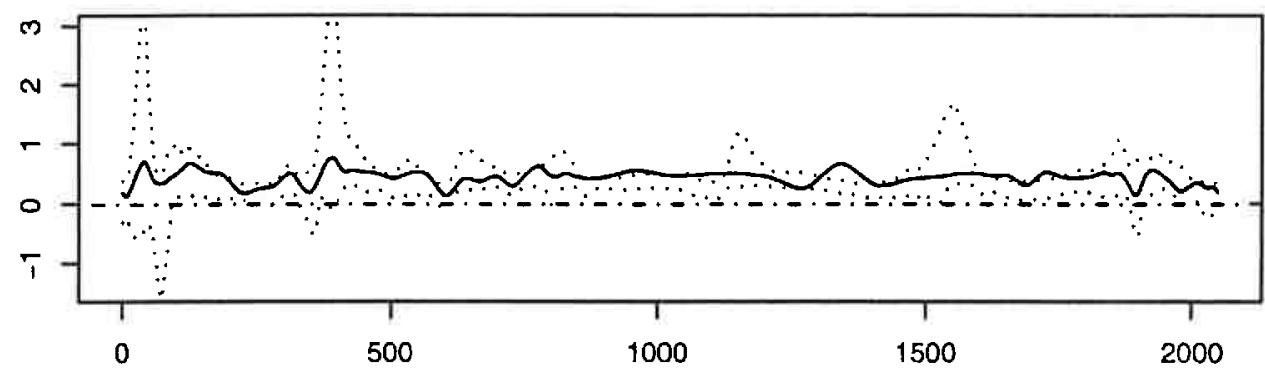

(a)

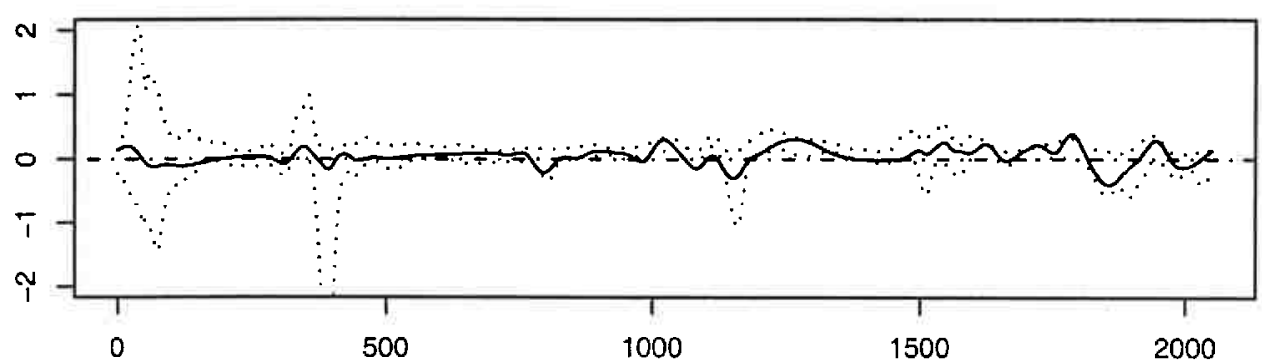

(b)

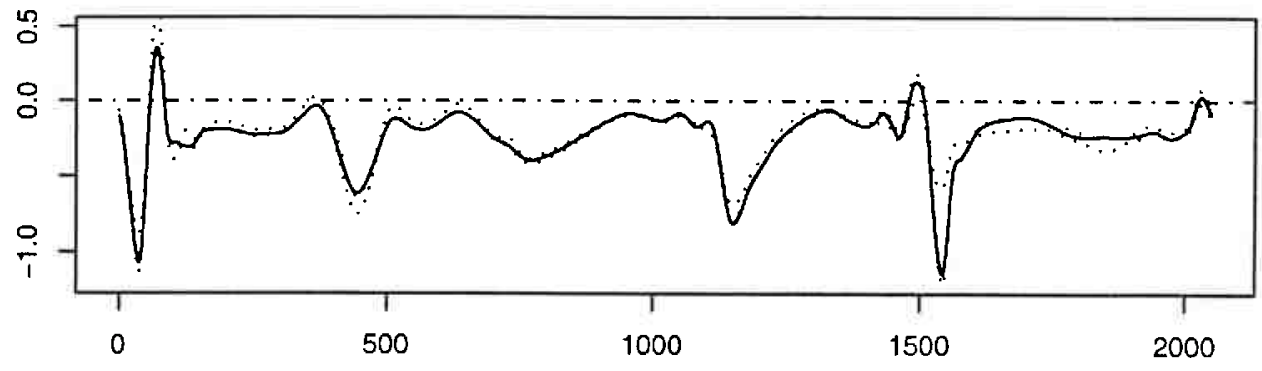

(c)

Figura 4.13: Resultados do ajuste do modelo 3, as Figuras (a), (b) e (c) são as estimativas $\tilde{\delta}_{1}^{(L)}(t), \tilde{\delta}_{2}^{(L)}(t)$ e $\tilde{\omega}_{0}^{(L)}(t)$ (linha cheia) e intervalo bootstrap de $95 \%$ (linha pontilhada). 
O critério para a escolha do modelo é o Erro Quadrático Médio da diferença entre $\tilde{Y}_{t, T}$ e $\tilde{Z}_{t, T}$, em que $\tilde{Z}_{t, T}$ é dado por:

para $M_{1}$,

$$
\tilde{Z}_{t, T}=\tilde{\omega}_{0}^{(L)}(t) \tilde{X}_{t, T}+\tilde{\delta}_{1}^{(L)}(t) \tilde{Y}_{t-1, T}, \quad t=2,3 \cdots, 2048
$$

para $M_{2}$,

$$
\tilde{Z}_{t, T}=\tilde{\omega}_{0}^{(L)}(t) \tilde{X}_{t, T}+\tilde{\omega}_{1}^{(L)}(t) \tilde{X}_{t-1, T}+\tilde{\delta}_{1}^{(L)}(t) \tilde{Y}_{t-1, T}, \quad t=2,3 \cdots, 2048
$$

$\operatorname{para} M_{3}$

$$
\tilde{Z}_{t, T}=\tilde{\omega}_{0}^{(L)}(t) \tilde{X}_{t, T}+\tilde{\delta}_{1}^{(L)}(t) \tilde{Y}_{t-1, T}+\tilde{\delta}_{2}^{(L)}(t) \tilde{Y}_{t-2, T}, \quad t=3,4, \cdots, 2048
$$

A diferença $\tilde{Y}_{t, T}-\tilde{Z}_{t, T}$ é um resíduo, porém não é ruído branco, uma vez que temos o ajuste $Y_{t, T}=\mathbf{v}_{t}(B) X_{t, T}+\epsilon_{t, T}$; como a função $\delta_{t}(B)$ é o denominador da função racional $\mathbf{v}_{t}(B)$, o erro $\epsilon_{t, T}$ será um processo de médias móveis com os mesmos coeficientes $\delta_{t}(B)$, quando simplificamos a equação por meio de multiplicação.

Abaixo temos a Tabela 4.1 que sintetiza esses resultados e as Figuras 4.14, 4.15 e 4.16 apresentam as correlações dos resíduos para os modelos $M_{1}, M_{2}$ e $M_{3}$, respectivamente.

Tabela 4.1: Resultados para os modelos ajustados.

\begin{tabular}{cc}
\hline Modelo & EQM \\
\hline$M_{1}$ & 0,0058 \\
$M_{2}$ & 0,0062 \\
$M_{3}$ & 0,0059 \\
\hline
\end{tabular}




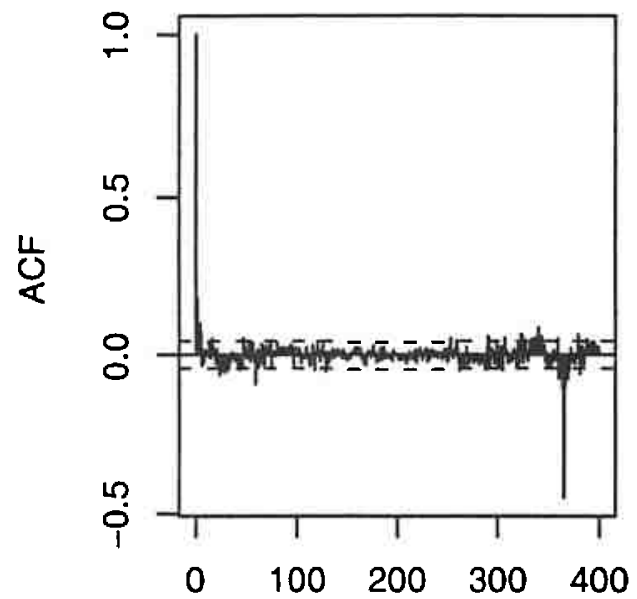

(a)

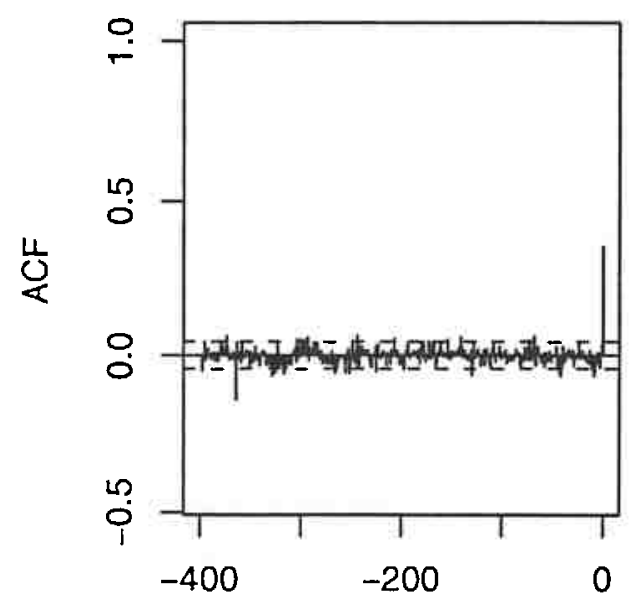

(c)

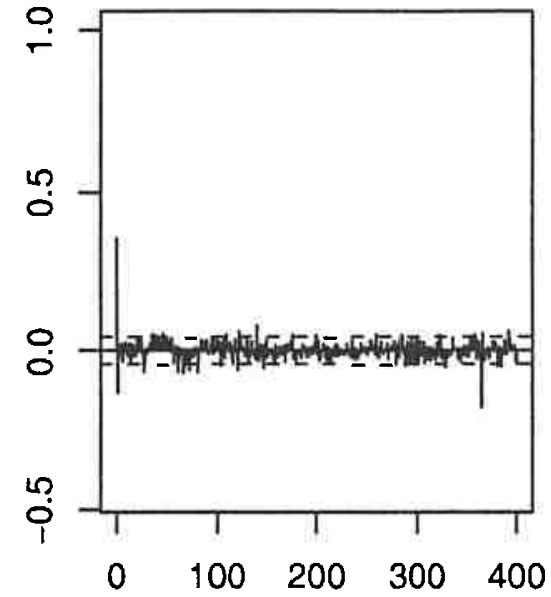

(b)

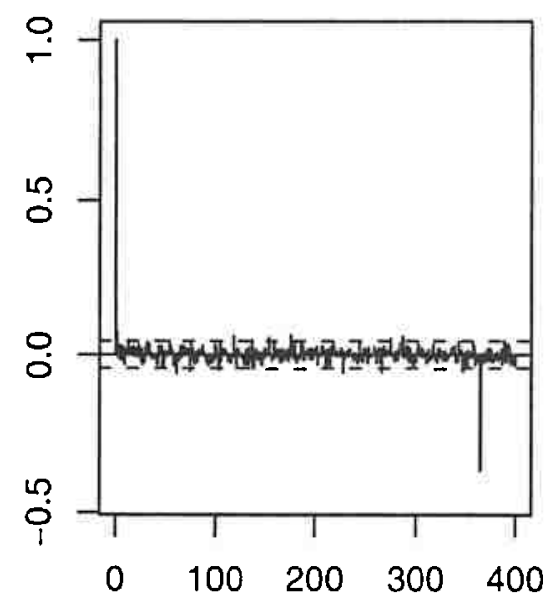

(d)

Figura 4.14: Correlações dos resíduos para o modelo 1. 

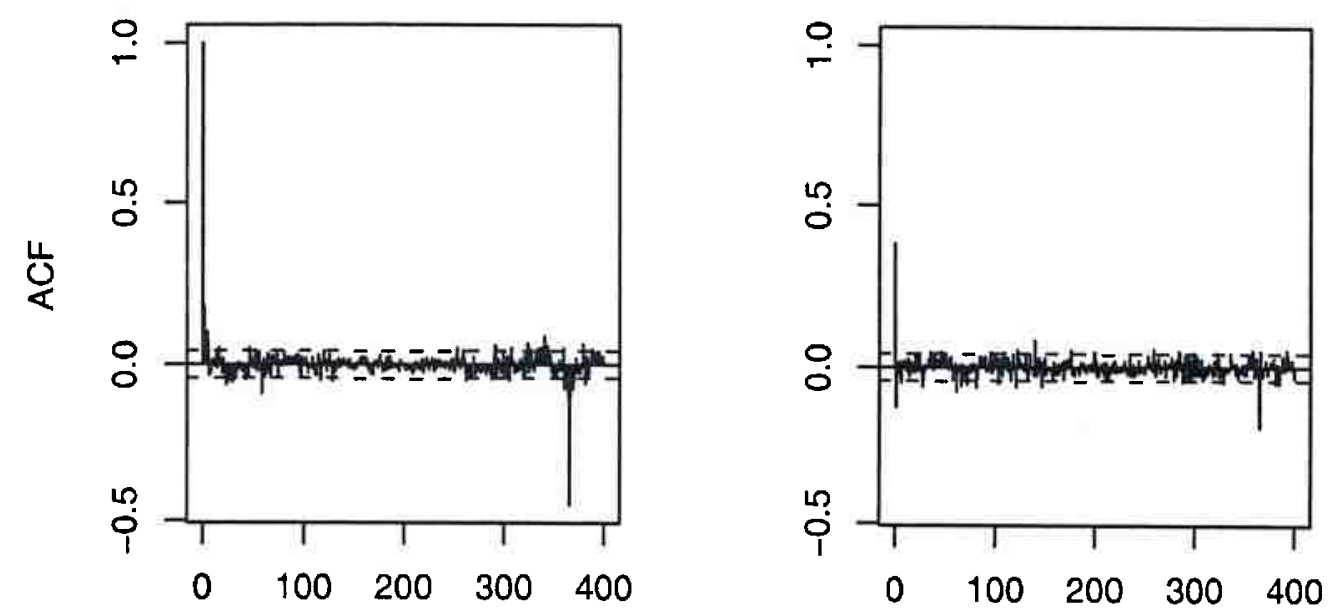

(a)

(b)
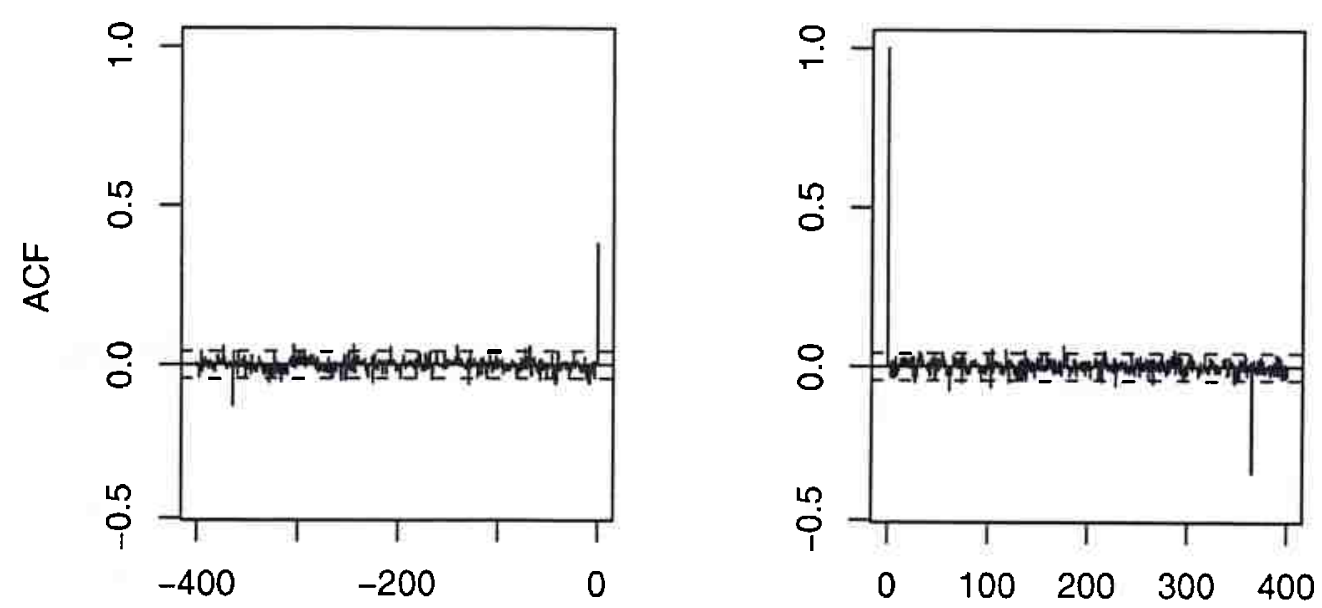

(c)

(d)

Figura 4.15: Correlações dos resíduos para o modelo 2. 


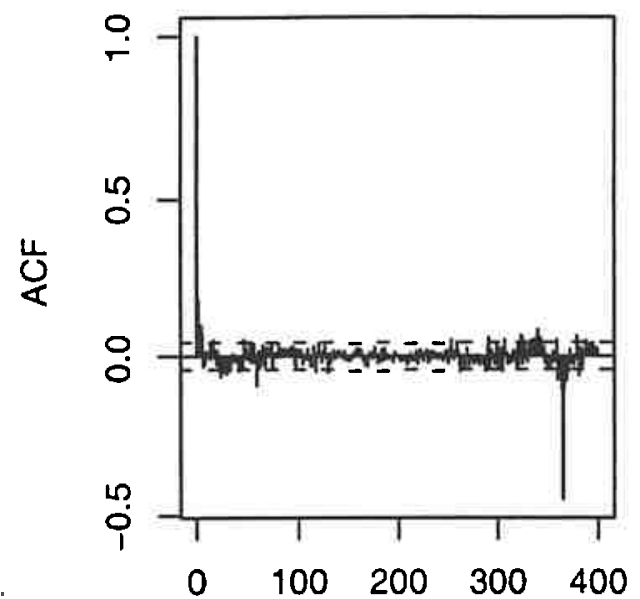

(9a)

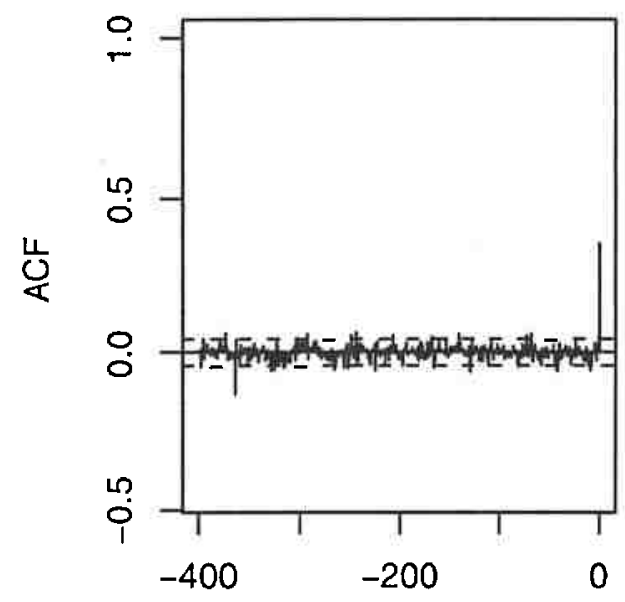

(c)

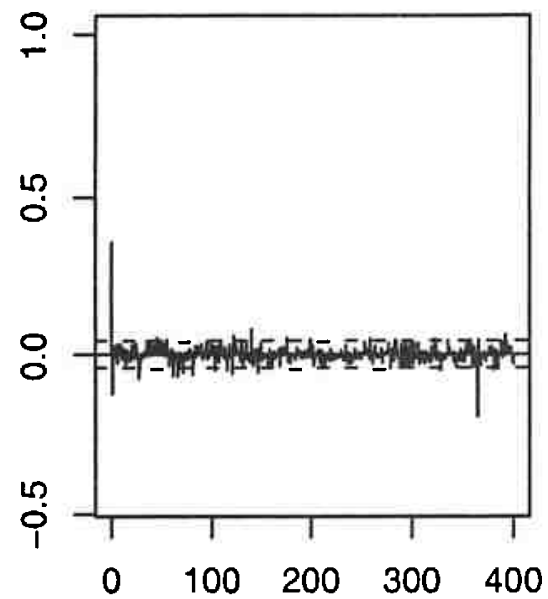

(b)

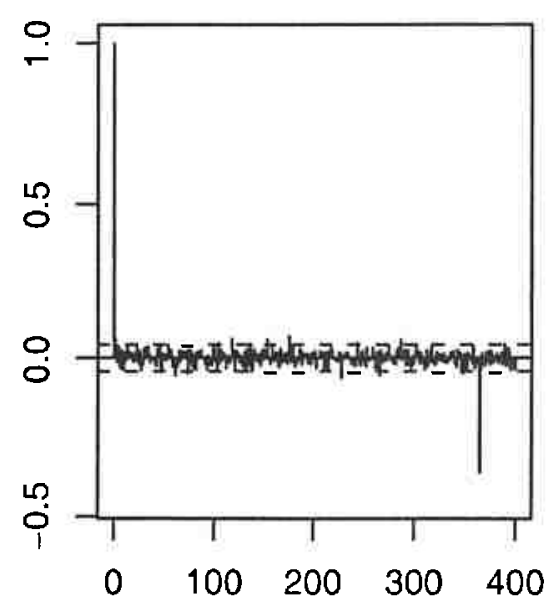

(d)

Figura 4.16: Correlações dos resíduos para o modelo 3. 
Especificamente as correlações dos resíduos consistem nas funções de autocorrelações dos resíduos (a), da função de correlação cruzada entre resíduos e a série de entrada (b), da função de correlação cruzada entre a série de entrada e resíduos (c) e da função de autocorrelação da série de entrada (d).

A correlações para as resíduos $\tilde{Y}_{t, T}-\tilde{Z}_{t, T}$, para os três modelos ajustados, comportam-se de maneira similar. Pela função de correlação cruzada vemos que ainda persiste uma correlação instantânea entre o resíduo e a série de entrada, $\tilde{X}_{t, T}$.

Dos três modelos em potencial, o modelo $M_{1}$ é mais parcimonioso, sendo portanto o preferível. As funções $\tilde{\omega}_{0}^{(L)}(t)$ e $\tilde{\delta}_{1}^{(L)}(t)$ têm comportamento semelhante nos três modelos, pois as funções $\tilde{\omega}_{1}^{(L)}(t)$ e $\tilde{\delta}_{2}^{(L)}(t)$ são estatisticamente nulas.

Os valores de $t$, que levaram $\tilde{\omega}_{0}^{(L)}(t)$ a assumir um ponto minimo local, correspondem a dias em que houve uma abrupta diminuição na temperatura máxima em um período sem chuva, como podemos ver na Figura 4.18. 


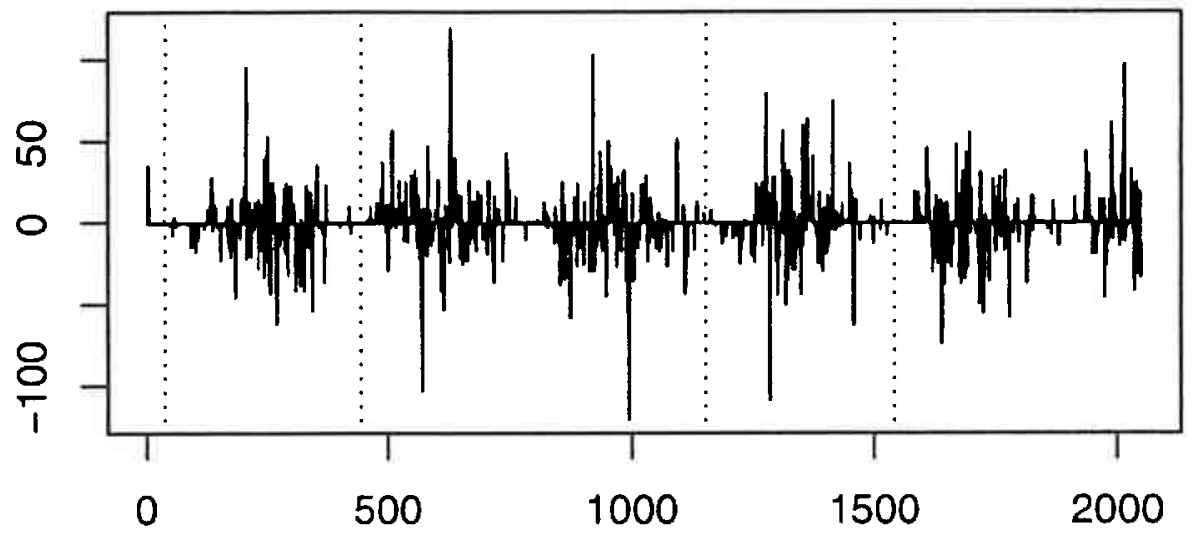

(a)

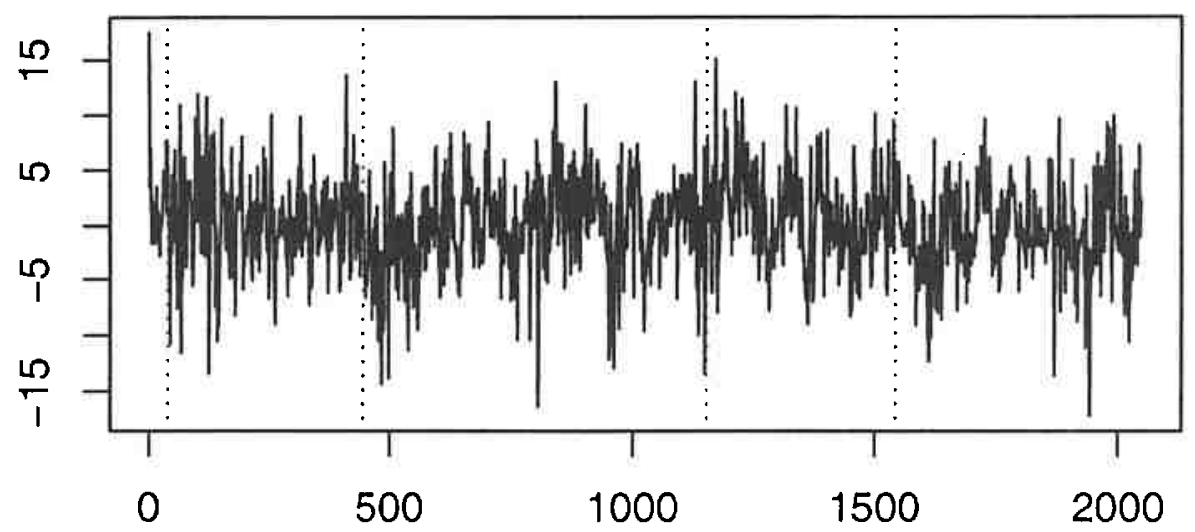

(b)

Figura 4.17: Séries de Entrada $\tilde{X}_{t, T}$ (a) e Saída $\tilde{Y}_{t, T}(\mathrm{~b})$ a linha pontilhada indica os valores de $t$ nos quais há picos em $\tilde{\omega}_{0}^{(L)}(t)$. 


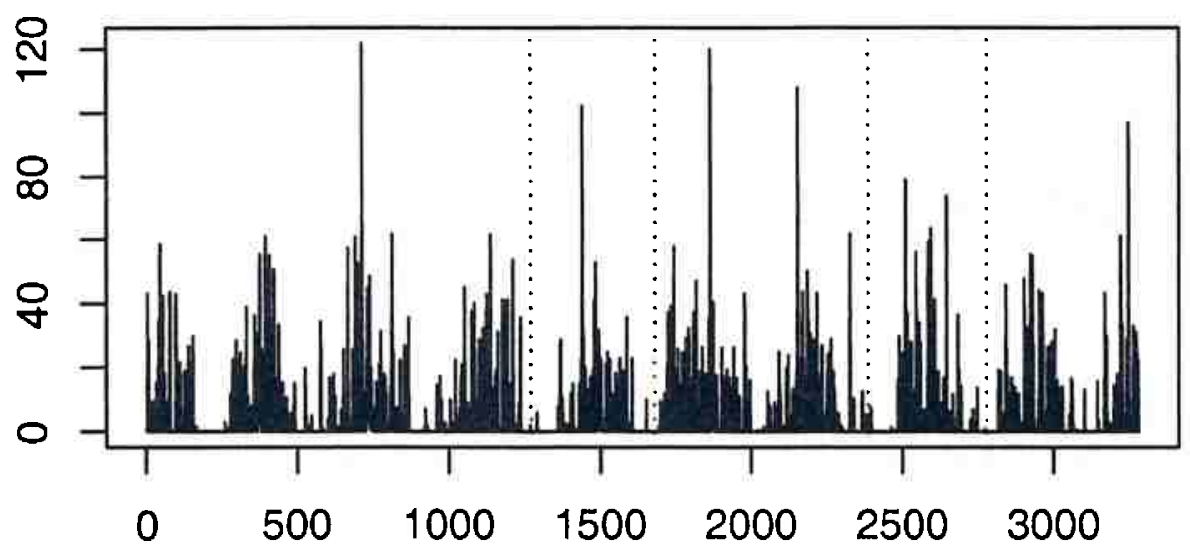

(a)

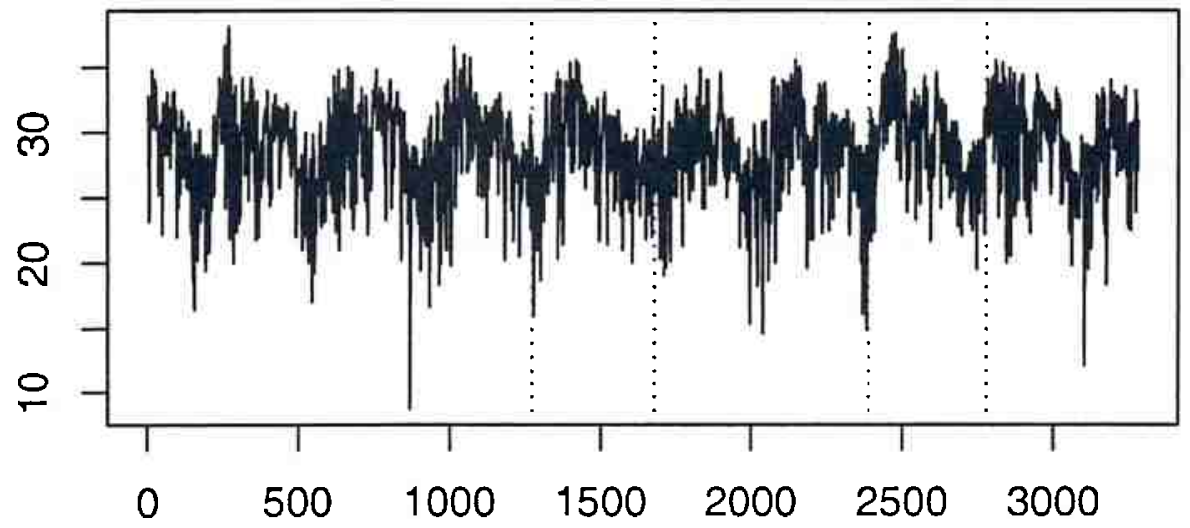

(b)

Figura 4.18: Séries de Entrada $X_{t, T}$ (a) e Saída $Y_{t, T}(\mathrm{~b})$ a linha pontilhada indica os valores de $t$ nos quais há picos em $\tilde{\omega}_{0}^{(L)}(t)$. 


\section{Capítulo 5}

\section{Conclusões e Trabalhos Futuros}

Neste trabalho apresentamos o modelo de função de transferência quando a série de entrada é localmente estacionária. Mostramos que, nesta situação, e usando as definições de processos localmente estacionários apresentada por Dahlhaus (1997), a série de saída será também localmente estacionária.

Para estimar os coeficientes da função de transferência, que neste caso, variam com o tempo, propusemos um estimador em dois estágios. Os dois estágios se mostraram necessários pois a estimativa do primeiro estágio se apresentou viesada, como constatamos pelos resultados das simulações apresentados no Capítulo 4 e no Apêndice C.

Em cada estágio fazemos uma regressão da série de saída sobre combinações lineares das série de entrada e defasagens da série de saída. As combinações lineares são obtidas através de decomposiçōes em ondaletas das séries. Mostramos que os coeficientes empíricos estimados, no primeiro estágio, têm distribuição assintoticamente normal. Por simulação verificamos que os coeficientes da função de transferência, que são obtidos através da transformada inversa de ondaletas dos 
coeficientes empíricos, também terão distribuição aproximadamente normal para todos os valores de $t$.

Para suavizar os estimadores das funções dos coeficientes da função de transferência usamos a técnica de limiarização dos estimadores dos coeficientes de ondaletas, por meio do limiar duro universal. Após esse passo, verificamos a distribuição dos estimadores dos coeficientes da função de transferência para os valores de $t=$ $1,2,3, \cdots, T$, utilizando simulações e constatamos que os mesmos apresentaram uma variabilidade menor.

No segundo estágio, por meio de simulações, verificamos propriedades similares às propriedades do primeiro estágio, porém sem viés na média.

$\mathrm{Na}$ aplicação aos dados reais vemos que os coeficientes ajustados variam realmente com o tempo e a relação entre precipitação atmosférica e temperatura se altera nos períodos em que houve estiagem acentuada.

Como trabalhos futuros pretendemos encontrar as propriedades estatísticas para os estimadores do segundo estágio, utilizar outras famílias de ondaletas, que não as periódicas, ajustar o modelo de função de transferência com mais de uma série de entrada e incorporar um modelo para o erro. 


\section{Apêndice A}

\section{Normas e Lemas}

\section{A.1 Resultados de Normas de Matrizes}

\section{A.1.1 Definições}

Seja A matriz $p \times q$, então:

(a) $\|\mathbf{A}\|_{1}=\max _{j} \sum_{i=1}^{p}\left|a_{i j}\right|$;

(b) $\|\mathbf{A}\|_{\infty}=\max _{i} \sum_{j=1}^{q}\left|a_{i j}\right|$;

(c) $\|\mathbf{A}\|_{2}=\left(\text { maior autovalor de } \mathbf{A}^{\prime} \mathbf{A}\right)^{1 / 2}$.

As normas acima recebem os nomes de norma 1, norma infinita e norma 2 (espectral), respectivamente. 


\section{A.1.2 Equivalência entre Normas}

(a) As normas 1, 2 e infinita, definidas anteriormente, são equivalentes no seguinte sentido: se uma seqüência de matrizes $\left\{\mathbf{A}_{i}\right\}$ converge para $\mathbf{A}_{\infty}$ em uma das normas, então essa seqüência de matrizes $\left\{\mathbf{A}_{i}\right\}$ converge em todas as três normas e as entradas das matrizes convergem para as entradas $\left[\mathbf{A}_{\infty}\right]_{u v}$.

(b) Para toda matriz A, $p \times q$ :

$$
\begin{gathered}
\frac{\|\mathbf{A}\|_{2}}{\sqrt{p}} \leq\|\mathbf{A}\|_{\infty} \leq\|\mathbf{A}\|_{2 \sqrt{q}} \\
\frac{\|\mathbf{A}\|_{2}}{\sqrt{q}} \leq\|\mathbf{A}\|_{1} \leq\|\mathbf{A}\|_{2 \sqrt{q}} \\
\frac{\|\mathbf{A}\|_{1}}{p} \leq\|\mathbf{A}\|_{\infty} \leq\|\mathbf{A}\|_{1} q .
\end{gathered}
$$

\section{A.2 Normalidade de Formas Quadráticas}

Seja a forma quadrática

$$
\eta_{T}=\mathbf{X}_{T}^{\prime} \mathbf{A X}_{T}
$$

$\operatorname{com} \mathbf{X}_{T}=\left(X_{1}, X_{2}, \ldots, X_{T}\right)^{\prime}$ e $\mathbf{A}=\left[\left(a_{i j}\right)\right] i, j=1,2, \ldots, T$, e $a_{i j}=a_{j i}$

Façamos

$$
\xi_{T}=\mathbf{Y}_{T}^{\prime} \mathrm{AY}_{T}
$$

sendo $\mathrm{Y}_{T}=\left(Y_{1}, Y_{2}, \ldots, Y_{T}\right)^{\prime}$ um vetor normal com média zero e mesma matriz de covariância de $\mathbf{X}_{T}$. 
Lema A.2.1: Seja $\boldsymbol{E} X_{t}=0$ e para algum $\gamma \geq 0$,

$$
\sup _{1 \leq t_{1} \leq T}\left\{\sum_{t_{2}, \ldots, t_{k}=1}^{T} \mid \operatorname{cum}\left(X_{t_{1}}, \ldots, X_{t_{k}} \mid\right\} \leq C^{k}(k !)^{1+\gamma}\right.
$$

para todo $T$ e $k=2,3, \ldots$ Então, para $n \geq 2$,

$$
\operatorname{cum}_{n}\left(\boldsymbol{\eta}_{T}\right)=\operatorname{cum}_{n}\left(\boldsymbol{\xi}_{T}\right)+R_{n}
$$

em que

(i) $\left|\operatorname{cum}_{n}\left(\boldsymbol{\xi}_{T}\right)\right| \leq \operatorname{var}\left(\boldsymbol{\xi}_{T}\right) 2^{n-2}(n-1) !\left[\lambda_{\max }(\mathbf{A}) \lambda_{\max }\left(\operatorname{cov}\left(\mathbf{Y}_{T}\right)\right)\right]^{n-2}$;

(ii) $R_{n} \leq 2^{n-2} C^{2 n}((2 n) !)^{1+\gamma} \max _{s, t}\left\{\left|a_{s t}\right|\right\} \tilde{\mathbf{A}}\|\mathbf{A}\|_{\infty}^{n-2}$, $\tilde{\mathbf{A}}=\sum_{s} \max _{t}\left\{\left(a_{s t}\right)\right\}$

$\|\mathbf{A}\|_{\infty}=\max _{s}\left\{\sum_{t}\left|a_{s t}\right|\right\}$.

A prova deste lema está em Neuman (1996).

Lema A.2.2: Suponha, para algum $\Delta_{T} \rightarrow 0$, que

$$
\left|\operatorname{cum}_{n}\left(\eta_{T} / \sqrt{\operatorname{var}\left(\eta_{T}\right)}\right)\right| \leq \frac{(n !)^{1+\gamma}}{\Delta_{T}^{n-2}}, \quad \text { para } \quad n=3,4, \cdots
$$

Então

$$
\frac{\mathbb{P}\left( \pm\left(\boldsymbol{\eta}_{T}-\boldsymbol{E} \boldsymbol{\eta}_{T}\right) / \sqrt{\operatorname{var}\left(\boldsymbol{\eta}_{T}\right)} \geq x\right)}{1-\Phi(x)} \rightarrow 1
$$

vale uniformemente sobre $0 \leq x \leq \nu_{T}$, onde $\nu_{T}=o\left(\Delta_{T}^{1 /(3+6 \gamma)}\right)$.

O Lema é de Rudzkis et al. (1978).

Lema A.2.3: Suponha, para algum $\bar{\Delta}_{T} \rightarrow 0$, que

$$
\left|\operatorname{cum}_{n}\left(\eta_{T}\right)\right| \leq\left(\frac{n !}{2}\right)^{1+\gamma} \frac{H_{T}}{\bar{\Delta}_{T}^{n-2}}, \quad \text { para } \quad n=3,4, \cdots
$$


Então, para $x \geq 0$,

$$
\begin{aligned}
P\left( \pm \eta_{T} \geq x\right) & \leq \exp \left(-\frac{x^{2}}{2\left[H_{T}+\left(x / \bar{\Delta}_{T}^{1 /(1+2 \gamma)}\right)^{(1+2 \gamma) /(1+\gamma)}\right]}\right) \\
& \leq\left\{\begin{array}{cc}
\exp \left(-x^{2} / 4 H_{T}\right), & \text { se } 0 \leq x \leq\left(H_{T}^{1+\gamma} \bar{\Delta}_{T}\right)^{1 /(1+2 \gamma)} \\
\exp \left(-\frac{1}{4}\left(x \bar{\Delta}_{T}\right)^{1 /(1+\gamma)}\right), & \text { se } x \geq\left(H_{T}^{1+\gamma} \bar{\Delta}_{T}\right)^{1 /(1+2 \gamma)}
\end{array}\right.
\end{aligned}
$$

O Lema é de Bentkus and Rudzkis (1980).

\section{A.3 Lemas}

Nos lemas apresentados a seguir, consideramos as Suposições (1)-(5) satisfeitas.

Seja $\Sigma_{t, T}=\operatorname{cov}\left(\left(Y_{t-1, T}, \cdots, Y_{t-r, T}, X_{t-b, T}, X_{t-b-1, T}, \cdots, X_{t-b-s, T}\right)^{\prime}\right)$.

Lema A.3.1: Pela suposição 3, com constantes $C_{1}, C_{2}>0$,

(i) $\lambda_{\max }\left(\Sigma_{t, T}\right) \leq C_{2}$ e $\lambda_{\min }\left(\Sigma_{t, T}\right) \geq C_{1}+o(1)$, em que $o(1)$ é uniforme em $t$;

(ii) Existe alguma função $g, \operatorname{com} g(s) \rightarrow 0$ se $s \rightarrow 0$, talque

$$
\left\|\Sigma_{t_{1}, T}-\Sigma_{t_{2}, T}\right\| \leq g\left(\frac{t_{1}-t_{2}}{T}\right) \quad \text { para todo } t_{1}, t_{2}, T
$$

(iii) $c(u, k-l)$ é uniformemente contínua em $u \mathrm{e}$

$$
\begin{array}{r}
\lim _{T \rightarrow \infty, t / T \rightarrow u} \operatorname{cov}\left(Y_{t-l, T}, Y_{t-k, T}\right)=c_{y y}(u, k-l), \\
\lim _{T \rightarrow \infty, t / T \rightarrow u} \operatorname{cov}\left(X_{t-l, T}, X_{t-k, T}\right)=c_{x x}(u, k-l), \\
\lim _{T \rightarrow \infty, t / T \rightarrow u} \operatorname{cov}\left(X_{t-l, T}, Y_{t-k, T}\right)=c_{x y}(u, k-l) e \\
\lim _{T \rightarrow \infty, t / T-u} \operatorname{cov}\left(Y_{t-l, T}, X_{t-k, T}\right)=c_{y x}(u, k-l) .
\end{array}
$$


Prova. Comecemos por (iii); pela representação (2.1) temos que

$$
X_{t, T}=\int_{-\pi}^{\pi} e^{i \lambda t} A_{X}\left(\frac{t}{T}, \lambda\right) d \xi(\lambda)
$$

em que $\xi(\lambda)$ é um processo com média zero e incrementos ortogonais, e por (2.4) e (2.5) temos que:

$$
Y_{t, T}=\int_{-\pi}^{\pi} e^{i \lambda t} A_{Y}\left(\frac{t}{T}, \lambda\right) d \xi(\lambda)+\int_{-\pi}^{\pi} e^{i \lambda t} \frac{1}{\sqrt{2 \pi}} d \zeta(\lambda)
$$

onde os processos $\xi(\lambda)$ e $\zeta(\lambda)$ são independentes e $A_{X}\left(\frac{t}{T}, \lambda\right)$ e $A_{Y}\left(\frac{t}{T}, \lambda\right)$ são uniformemente contínuas em $u=t / T, t=1,2,3, \cdots, T$.

Consideremos o vetor de variáveis explicativas, $\widetilde{X}_{t, T}=\left[Y_{t-1, T}, \cdots, Y_{t-r, T}\right.$, $\left.X_{t-b, T}, X_{t-b-1, T}, \cdots, X_{t-b-s, T}\right]^{\prime}$. Então,

$$
\begin{aligned}
\operatorname{cov}\left(Y_{t-l, T}, Y_{t-k, T}\right)= & \int_{-\pi}^{\pi} e^{i \lambda(t-l)} A_{Y}\left(\frac{t-l}{T}, \lambda\right) e^{-i \lambda(t-k)} A_{Y}\left(\frac{t-k}{T},-\lambda\right) d \xi(\lambda)+ \\
& +\int_{-\pi}^{\pi} e^{i \lambda(t-l)} \frac{1}{\sqrt{2 \pi}} e^{-i \lambda(t-k)} \frac{1}{\sqrt{2 \pi}} d \zeta(\lambda)+o(1) \\
= & \int_{-\pi}^{\pi} e^{i \lambda(k-l)}\left|A_{Y}\left(\frac{t}{T}, \lambda\right)\right|^{2} d \xi(\lambda)+\int_{-\pi}^{\pi} e^{i \lambda(k-l)} \frac{1}{2 \pi} d \zeta(\lambda)+o(1) \\
= & c_{y, y}(u, k-l)+o(1), \\
\operatorname{cov}\left(X_{t-l, T}, X_{t-k, T}\right)= & \int_{-\pi}^{\pi} e^{i \lambda(k-l)}\left|A_{X}\left(\frac{t}{T}, \lambda\right)\right|^{2} d \xi(\lambda)=c_{x, x}(u, k-l)+o(1), \\
\operatorname{cov}\left(X_{t-l, T}, Y_{t-k, T}\right)= & \int_{-\pi}^{\pi} e^{i \lambda(k-l) \mid}\left|A_{X}\left(\frac{t}{T}, \lambda\right) A_{Y}\left(\frac{t}{T}, \lambda\right)\right| d \xi(\lambda)=c_{x, y}(u, k-l)+o(1), \\
\operatorname{cov}\left(Y_{t-l, T}, X_{t-k, T}\right)= & \int_{-\pi}^{\pi} e^{i \lambda(k-l) \mid}\left|A_{Y}\left(\frac{t}{T}, \lambda\right) A_{X}\left(\frac{t}{T}, \lambda\right)\right| d \xi(\lambda)=c_{y, x}(u, k-l)+o(1),
\end{aligned}
$$

as funções são uniformente contínuas, $t / T \rightarrow u$. o que implica (iii).

(ii) Como $\Sigma_{t, T}=\operatorname{cov}\left(\left(Y_{t-1, T}, \cdots, Y_{t-r, T}, X_{t-b, T}, X_{t-b-1, T}, \cdots, X_{t-b-s, T}\right)^{\prime}\right), \Sigma_{t, T}$ pode ser escrita como:

$$
\Sigma_{t, T}=\left[\begin{array}{cc}
\Sigma_{t, T}^{Y Y} & \Sigma_{t, T}^{Y X} \\
\Sigma_{t, T}^{X Y} & \Sigma_{t, T}^{X X}
\end{array}\right]
$$


A submatriz $\Sigma_{t, T}^{Y Y}$ é formada por $c_{y y}(u, k-l), u=t / T, t=\max \{r, s+b\}, \ldots, T$, em que $k-l$ é a defasagem entre as observações. As outras três submatrizes de $\Sigma_{t, T}$ são semelhantes a $\Sigma_{t, T}^{Y Y}$. Dada a continuidade de $A_{Y}\left(\frac{t}{T}, \lambda\right)$ e de $A_{X}\left(\frac{t}{T}, \lambda\right)$, temos que

$$
\left\|\Sigma_{t_{1}, T}-\Sigma_{t_{2}, T}\right\| \leq g\left(\frac{t_{1}-t_{2}}{T}\right), \quad \text { para todo } t_{1}, t_{2}, T
$$

o que prova $(i i)$.

(i) Como $A_{Y}\left(\frac{t}{T}, \lambda\right)$ e $A_{X}\left(\frac{t}{T}, \lambda\right)$ são periódicas, o que implica que são funções limitadas, temos que $\lambda_{\max }\left(\Sigma_{t, T}\right) \leq C_{2}$ e $\lambda_{\min }\left(\Sigma_{t, T}\right) \geq C_{1}$, o que finaliza a prova.

Lema A.3.2: Suponha agora que a suposição (6) também seja válida, e faça $0<\varrho_{1}<\varrho$. Então

$$
\boldsymbol{E}\left|\hat{\beta}_{j k}^{(i)}-\beta_{j k}^{(i)}\right|^{2+\varrho_{1}}=O(1)
$$

vale uniformemente em $i, k$ e $j<j^{*}$.

Prova. Encontraremos aqui as estimativas para os momentos de $\left\|\boldsymbol{\Upsilon}^{\prime} \epsilon\right\|$ e $\left\|\boldsymbol{\Upsilon}^{\prime} \mathbf{S}\right\|$ que serão usadas mais adiante.

Através da representação $M A(\infty)$ de $\left\{X_{t, T}\right\}$ e $\left\{Y_{t, T}\right\}$, podemos escrever $\left(\Upsilon^{\prime} \boldsymbol{\epsilon}\right)_{v}$, isto é a $v$-ésima posição do vetor $\Upsilon^{\prime} \epsilon$, como uma forma quadrática $\boldsymbol{\epsilon}^{\prime} A \boldsymbol{\epsilon}$ para alguma matriz $A=A(p, k)$, em que $\epsilon=\left[\epsilon_{T}, \epsilon_{T-1}, \cdots, \epsilon_{1}, \epsilon_{0}, \epsilon_{-1}, \cdots\right]^{\prime}$ é um vetor infinito-dimensional.

A matriz $\Upsilon^{\prime}$ está dada nas equações (3.15) e (3.16), e dado que a prova do Lema A.2.1 não depende da dimensão da matriz $A$, podemos aplicar esse lema ao caso infinito dimensional. 
Usando a notação do Lema A.2.1 (ii), temos

$$
\begin{array}{r}
\tilde{A}=O\left(2^{-j^{*} / 2} T\right), \\
\max \left\{\left|a_{s, t}\right|\right\} \leq\|\widetilde{A}\|_{\infty}=O\left(2^{j^{*} / 2}\right)
\end{array}
$$

No caso mais simples, $r=1$ e $s=b=0$ teremos que $\Upsilon^{\prime}$ e será

$$
\mathbf{\Upsilon}^{\prime} \mathbf{e}=\left[\begin{array}{c}
\sum_{t=2}^{T} \phi_{j^{*}, 1}\left(\frac{t}{T}\right) Y_{t-1, T} e_{t, T} \\
\sum_{t=2}^{T} \phi_{j^{*}, 2}\left(\frac{t}{T}\right) Y_{t-1, T} e_{t, T} \\
\vdots \\
\sum_{t=2}^{T} \phi_{j^{*}, 2^{*}}\left(\frac{t}{T}\right) Y_{t-1, T} e_{t, T} \cdots \\
\sum_{t=2}^{T} \phi_{j^{*}, 1}\left(\frac{t}{T}\right) X_{t, T} e_{t, T} \\
\sum_{t=2}^{T} \phi_{j^{*}, 2}\left(\frac{t}{T}\right) X_{t, T} e_{t, T} \\
\vdots \\
\sum_{t=2}^{T} \phi_{j^{*}, 2^{*}}\left(\frac{t}{T}\right) X_{t, T} e_{t, T}
\end{array}\right],
$$

$\operatorname{mas} e_{t, T}=\epsilon_{t}-\delta_{1}(t) \epsilon_{t-1}$, então,

$$
\mathbf{\Upsilon}^{\prime} \mathbf{e}=\left[\begin{array}{c}
\sum_{t=2}^{T} \phi_{j^{*}, 1}\left(\frac{t}{T}\right) Y_{t-1, T} \epsilon_{t} \\
\sum_{t=2}^{T} \phi_{j^{*}, 2}\left(\frac{t}{T}\right) Y_{t-1, T} \epsilon_{t} \\
\vdots \\
\sum_{t=2}^{T} \phi_{j^{*}, 2^{j^{*}}}\left(\frac{t}{T}\right) Y_{t-1, T} \epsilon_{t} \\
\cdots \\
\sum_{t=2}^{T} \phi_{j^{*}, 1}\left(\frac{t}{T}\right) X_{t, T} \epsilon_{t} \\
\sum_{t=2}^{T} \phi_{j^{*}, 2}\left(\frac{t}{T}\right) X_{t, T} \epsilon_{t} \\
\vdots \\
\sum_{t=2}^{T} \phi_{j^{*}, 2^{*}}\left(\frac{t}{T}\right) X_{t, T} \epsilon_{t}
\end{array}\right]-\left[\begin{array}{c}
\sum_{t=2}^{T} \phi_{j^{*}, 1}\left(\frac{t}{T}\right) Y_{t-1, T} \delta_{1}(t) \epsilon_{t-1} \\
\sum_{t=2}^{T} \phi_{j^{*}, 2}\left(\frac{t}{T}\right) Y_{t-1, T} \delta_{1}(t) \epsilon_{t-1} \\
\vdots \\
\sum_{t=2}^{T} \phi_{j^{*}, 2^{*}}\left(\frac{t}{T}\right) Y_{t-1, T} \delta_{1}(t) \epsilon_{t,-1 T} \\
\cdots \\
\sum_{t=2}^{T} \phi_{j^{*}, 1}\left(\frac{t}{T}\right) X_{t, T} \delta_{1}(t) \epsilon_{t-1} \\
\sum_{t=2}^{T} \phi_{j^{*}, 2}\left(\frac{t}{T}\right) X_{t, T} \delta_{1}(t) \epsilon_{t-1} \\
\vdots \\
\sum_{t=2}^{T} \phi_{j^{*}, 2^{*}}\left(\frac{t}{T}\right) X_{t, T} \delta_{1}(t) \epsilon_{t-1}
\end{array}\right]
$$

Usando a representação $M A(\infty)$ de $\left\{X_{t, T}\right\}$ e $\left\{Y_{t, T}\right\}$ temos

$$
X_{t, T}=\sum_{s=0}^{\infty} \gamma_{t, T}^{X}(s) a_{t-s}
$$




$$
\begin{aligned}
& Y_{t, T}=\sum_{s=0}^{\infty} \gamma_{t, T}^{Y}(s) a_{t-s}+\epsilon_{t} \\
& \mathbf{\Upsilon}^{\prime} \mathbf{e}=\left[\begin{array}{c}
\sum_{t=2}^{T} \phi_{j^{*}, 1}\left(\frac{t}{T}\right)\left(\sum_{s=0}^{\infty} \gamma_{t-1, T}^{Y}(s) a_{t-1-s}+\epsilon_{t}\right) \epsilon_{t} \\
\sum_{t=2}^{T} \phi_{j^{*}, 2}\left(\frac{t}{T}\right)\left(\sum_{s=0}^{\infty} \gamma_{t-1, T}^{Y}(s) a_{t-1-s}+\epsilon_{t}\right) \epsilon_{t} \\
\vdots \\
\sum_{t=2}^{T} \phi_{j^{*}, 2^{*}}\left(\frac{t}{T}\right)\left(\sum_{s=0}^{\infty} \gamma_{t-1, T}^{Y}(s) a_{t-1-s}+\epsilon_{t}\right) \epsilon_{t} \\
\ldots \\
\sum_{t=2}^{T} \phi_{j^{*}, 1}\left(\frac{t}{T}\right)\left(\sum_{s=0}^{\infty} \gamma_{t, T}^{X}(s) a_{t-1-s}\right) \epsilon_{t} \\
\sum_{t=2}^{T} \phi_{j^{*}, 2}\left(\frac{t}{T}\right)\left(\sum_{s=0}^{\infty} \gamma_{t, T}^{X}(s) a_{t-1-s}\right) \epsilon_{t} \\
\vdots \\
\sum_{t=2}^{T} \phi_{j^{*}, 2^{*}}\left(\frac{t}{T}\right)\left(\sum_{s=0}^{\infty} \gamma_{t, T}^{X}(s) a_{t-1-s}\right) \epsilon_{t}
\end{array}\right]+ \\
& -\left[\begin{array}{c}
\sum_{t=2}^{T} \phi_{j^{*}, 1}\left(\frac{t}{T}\right)\left(\sum_{s=0}^{\infty} \gamma_{t-1, T}^{Y}(s) a_{t-1-s}+\epsilon_{t}\right) \delta_{1}(t) \epsilon_{t-1} \\
\sum_{t=2}^{T} \phi_{j^{*}, 2}\left(\frac{t}{T}\right)\left(\sum_{s=0}^{\infty} \gamma_{t-1, T}^{Y}(s) a_{t-1-s}+\epsilon_{t}\right) \delta_{1}(t) \epsilon_{t-1} \\
\vdots \\
\sum_{t=2}^{T} \phi_{j^{*}, 2^{j^{*}}}\left(\frac{t}{T}\right)\left(\sum_{s=0}^{\infty} \gamma_{t-1, T}^{Y}(s) a_{t-1-s}+\epsilon_{t}\right) \delta_{1}(t) \epsilon_{t-1} \\
\cdots \\
\sum_{t=2}^{T} \phi_{j^{*}, 1}\left(\frac{t}{T}\right)\left(\sum_{s=0}^{\infty} \gamma_{t, T}^{X}(s) a_{t-1-s}\right) \delta_{1}(t) \epsilon_{t-1} \\
\sum_{t=2}^{T} \phi_{j^{*}, 2}\left(\frac{t}{T}\right)\left(\sum_{s=0}^{\infty} \gamma_{t, T}^{X}(s) a_{t-1-s}\right) \delta_{1}(t) \epsilon_{t-1} \\
\vdots \\
\sum_{t=2}^{T} \phi_{j^{*}, 2^{*}}\left(\frac{t}{T}\right)\left(\sum_{s=0}^{\infty} \gamma_{t, T}^{X}(s) a_{t-1-s}\right) \delta_{1}(t) \epsilon_{t-1}
\end{array}\right]
\end{aligned}
$$

Assim, $\boldsymbol{\Upsilon}^{\prime} \mathbf{e}$ pode ser escrito como uma soma de formas quadráticas.

$$
\mathbf{\Upsilon}^{\prime} \mathbf{e}=\left[\begin{array}{ll}
\mathrm{a} & \epsilon_{1}
\end{array}\right] \mathbf{A}_{1}\left[\begin{array}{l}
\epsilon_{1} \\
\epsilon_{2}
\end{array}\right]+\left[\begin{array}{ll}
\mathrm{a} & \epsilon_{2}
\end{array}\right] \mathbf{A}_{2}\left[\begin{array}{c}
\epsilon_{1} \\
\epsilon_{2}
\end{array}\right]
$$

em que $\mathbf{a}=\left[a_{t-1}, a_{t-2}, a_{t-3}, \cdots\right], \epsilon_{1}=\left[\epsilon_{t}, \epsilon_{t-1}, \cdots, \epsilon_{1}, \epsilon_{0}, \epsilon_{-1}, \cdots\right]^{\prime}$ e $\epsilon_{2}=\left[\epsilon_{t-1}, \epsilon_{t-2}, \cdots\right.$, $\left.\epsilon_{1}, \epsilon_{0}, \epsilon_{-1}, \cdots\right]^{\prime}$. 
Para cada matriz $A_{i}, i=1,2$ da forma quadrática (A.3), temos, usando a notação do Lema A.2.1, que

$$
\widetilde{A}_{i}=O\left(2^{-j^{*} / 2} T\right)
$$

e

$$
\max \left\{a_{i_{s t}}\right\} \leq\left\|A_{i}\right\|_{\infty}=O\left(2^{j^{*} / 2}\right)
$$

Como as variáveis do vetor $a$ são independentes de $\epsilon_{1}$ temos que

$$
\left|c u m_{n}\left(\mathbf{\Upsilon}^{\prime} \mathbf{e}\right)_{v}\right| \leq C^{n}(n !)^{(2+2 \gamma)} T\left(2^{j^{*} / 2}\right)^{n-2}, \quad \text { para } n \geq 2
$$

em que $v$ indica o compomente do vetor $\mathbf{\Upsilon}^{\prime} \mathbf{e}, v=1,2, \cdots,(r+s+b+1) * 2^{j^{*}}$.

Calcularemos agora $\mathbb{E}\left(\boldsymbol{\Upsilon}^{\prime} \mathbf{e}\right)_{v}$.

$$
\begin{aligned}
\mathbb{E}\left(\mathbf{\Upsilon}^{\prime} \mathbf{e}\right)_{v} & =\left[\sum_{t=2}^{T} \phi_{j^{*}, v}\left(\frac{t}{T}\right) Y_{t-1, T} \epsilon_{t}-\sum_{t=2}^{T} \phi_{j^{*}, v}\left(\frac{t}{T}\right) Y_{t-1, T} \delta_{1}(t) \epsilon_{t-1}\right] \\
& =-\sum_{t=2}^{T} \phi_{j^{*}, v}\left(\frac{t}{T}\right) \delta_{1}(t) \mathbb{E}\left[Y_{t-1, T} \epsilon_{t-1}\right]
\end{aligned}
$$

pois $\epsilon_{t}$ é ruído branco, portanto independe de $Y_{t-1, T}$ e temos que $\epsilon_{t}$ independe da série de entrada $X_{t, T}$.

$$
\mathbb{E}\left(Y_{t-1, T}^{r} \epsilon_{t-1}\right)=\mathbb{E}\left[\left(\frac{\omega_{0}(t-1)}{1-\delta_{1}(t-1) B} X_{t-1, T}+\epsilon_{t-1}\right) \epsilon_{t-1}\right]=\sigma^{2}
$$


Assim,

$$
\boldsymbol{E}\left(\boldsymbol{\Upsilon}^{\prime} \mathbf{e}\right)=\left[\begin{array}{c}
-\sum_{t=2}^{T} \phi_{j^{*}, 1}\left(\frac{t}{T}\right) \delta_{1}(t) \sigma^{2} \\
-\sum_{t=2}^{T} \phi_{j^{*}, 2}\left(\frac{t}{T}\right) \delta_{1}(t) \sigma^{2} \\
\vdots \\
-\sum_{t=2}^{T} \phi_{j^{*}, 2^{*}}\left(\frac{t}{T}\right) \delta_{1}(t) \sigma^{2} \\
\cdots \\
0 \\
0 \\
\vdots \\
0
\end{array}\right]=\mathbf{E}
$$

Assim, obtemos para $h$ ímpar, que:

$\mathbb{E}\left|\left(\mathbf{\Upsilon}^{\prime} \mathbf{e}\right)_{u}-\mathbf{E}_{u}\right|^{h}=O\left(\sum_{r=1}^{n} \prod_{i_{1}, \cdots, i_{r} ; i_{1}+\ldots+i_{r}=n ; i_{j} \geq 1}\left|c u m_{i_{j}}\left(\left(\mathbf{\Upsilon}^{\prime} \mathbf{e}\right)_{u}-\mathbf{E}_{u}\right)\right|\right) \leq C(h) T^{h / 2}$

Obtemos, com $\Delta=O\left(2^{j^{*}}\right)$

$$
\begin{aligned}
\mathbb{E}\left\|\left(\boldsymbol{\Upsilon}^{\prime} \mathbf{e}\right)_{u}-\mathbf{E}_{u}\right\|^{h} & =\mathbb{E}\left(\sum_{v}\left(\boldsymbol{\Upsilon}^{\prime} \mathbf{e}-\mathbf{E}\right)_{v}\right)^{h / 2} \\
& =(\Delta(r+s+1))^{h / 2-1} \sum_{v} \boldsymbol{E}\left(\boldsymbol{\Upsilon}^{\prime} \mathbf{e}-\mathbf{E}\right)_{v}^{2} \\
& =O\left((\Delta(r+s+1))^{h / 2} \max _{v}\left\{\boldsymbol{E}\left(\boldsymbol{\Upsilon}^{\prime} \mathbf{e}-\mathbf{E}\right)_{v}^{h}\right\}\right) \\
& =O\left(2^{j^{*} h / 2} T^{h / 2}\right)
\end{aligned}
$$

em que $v$ indica a posição do vetor $\left(\Upsilon^{\prime} \mathbf{e}-\mathbf{E}\right)_{u}, v=1,2,3, \cdots,(r+s+1) .2^{j^{*}}$. Estamos trabalhando com o caso mais simples, $r=1, s=0$. Analogamente, a quantidade || $\mathbf{\Upsilon}^{\prime} \mathbf{S} \|$ será 


$$
\mathbf{Y}^{\prime} \mathbf{S}=\left[\begin{array}{c}
\sum_{t=2}^{T} \phi_{j^{*}, 1}\left(\frac{t}{T}\right) \sum_{j \geq j^{*}} \sum_{k \in \mathcal{I}_{j}} \beta_{j k}^{\left(\delta_{1}\right)} \psi_{j k}\left(\frac{t}{T}\right) Y_{t-1, T} Y_{t-1, T} \\
\sum_{t=2}^{T} \phi_{j^{*}, 2}\left(\frac{t}{T}\right) \sum_{j \geq j^{*}} \sum_{k \in \mathcal{I}_{j}} \beta_{j k}^{\left(\delta_{1}\right)} \psi_{j k}\left(\frac{t}{T}\right) Y_{t-1, T} Y_{t-1, T} \\
\vdots \\
\sum_{t=2}^{T} \phi_{j^{*}, 2^{j^{*}}}\left(\frac{t}{T}\right) \sum_{j \geq j^{*}} \sum_{k \in \mathcal{I}_{j}} \beta_{j k}^{\left(\delta_{1}\right)} \psi_{j k}\left(\frac{t}{T}\right) Y_{t-1, T} Y_{t-1, T} \\
\ldots \\
\sum_{t=2}^{T} \phi_{j^{*}, 1}\left(\frac{t}{T}\right) \sum_{j \geq j^{*}} \sum_{k \in \mathcal{I}_{j}} \beta_{j k}^{\left(\delta_{1}\right)} \psi_{j k}\left(\frac{t}{T}\right) X_{t, T} Y_{t-1, T} \\
\sum_{t=2}^{T} \phi_{j^{*}, 2}\left(\frac{t}{T}\right) \sum_{j \geq j^{*}} \sum_{k \in \mathcal{I}_{j}} \beta_{j k}^{\left(\delta_{1}\right)} \psi_{j k}\left(\frac{t}{T}\right) X_{t, T} Y_{t-1, T} \\
\vdots \\
\sum_{t=2}^{T} \phi_{j^{*}, 2^{j^{*}}}\left(\frac{t}{T}\right) \sum_{j \geq j^{*}} \sum_{k \in \mathcal{I}_{j}} \beta_{j k}^{\left(\delta_{1}\right)} \psi_{j k}\left(\frac{t}{T}\right) X_{t, T} Y_{t-1, T}
\end{array}\right]+
$$

Da mesma maneira que no caso anterior, temos que, $\Upsilon^{\prime} \mathbf{S}$ pode ser escrito como uma soma de formas quadráticas.

$$
\mathbf{Y}^{\prime} \mathbf{S}=\left[\begin{array}{ll}
\mathbf{Y} & \mathbf{X}
\end{array}\right] \mathbf{A}_{1}\left[\begin{array}{c}
\mathbf{Y} \\
\mathbf{Y}
\end{array}\right]+\left[\begin{array}{ll}
\mathbf{Y} & \mathbf{X}
\end{array}\right] \mathbf{A}_{2}\left[\begin{array}{c}
\mathbf{X} \\
\mathbf{X}
\end{array}\right]
$$

Pela forma de $\mathbf{A}_{1}$, vemos que satisfaz

$$
\tilde{A}_{1}=O\left(\sum_{t=2}^{T}\left|\phi_{j^{*}, u}(t / T)\right| \sum_{j \geq j^{*}} \sum_{k \in \mathcal{I}_{j}}\left|\beta_{j k}^{\left(\delta_{1}\right)} \psi_{j k}\left(\frac{t}{T}\right)\right|\right)
$$




$$
\begin{aligned}
& =O\left(\sqrt{\sum_{t=2}^{T}\left(\phi_{j^{*}, u}(t / T)\right)^{2}} \sqrt{\sum_{t=2}^{T}\left(\sum_{j \geq j^{*}} \sum_{k \in \mathcal{I}_{j}}\left(\beta_{j k}^{\left(\delta_{1}\right)} \psi_{j k}(t / T)\right)\right)^{2}}\right) \\
& =O\left(T\left(2^{-2 j^{*} \min \left\{\bar{s}_{i}\right\}}+T^{-1 / 2} 2^{-j^{*} \min \left\{m_{i}-1 / 2-1 /\left(2 p_{i}\right)\right\}}\right)\right)=O\left(T^{1 / 2}\right) .
\end{aligned}
$$

Pela equação (A.10) de Dahlhaus et al. (1999) temos que

$$
\left\|A_{1}\right\|_{\infty}=O\left(2^{j^{*} / 2}\left\|\sum_{j \geq j^{*}} \sum_{k \in \mathcal{I}_{j}} \beta_{j k}^{\left(\delta_{1}\right)} \psi_{j k}\left(\frac{t}{T}\right)\right\|_{\infty}\right)=O\left(2^{j^{*} / 2}\right) .
$$

Para $A_{2}$ temos que

$$
\begin{aligned}
\widetilde{A}_{2} & =O\left(\sum_{t=2}^{T}\left|\phi_{j^{*}, u}(t / T)\right| \sum_{j \geq j^{*}} \sum_{k \in \mathcal{I}_{j}}\left|\beta_{j k}^{\left(\omega_{0}\right)} \psi_{j k}(t / T)\right|\right) \\
& =O\left(\sqrt{\sum_{t=2}^{T}\left(\phi_{j^{*}, u}(t / T)\right)^{2}} \sqrt{\sum_{t=2}^{T}\left(\sum_{j \geq j^{*}} \sum_{k \in \mathcal{I}_{j}}\left(\beta_{j k}^{\left(\omega_{0}\right)} \psi_{j k}(t / T)\right)\right)^{2}}\right) \\
& =O\left(T\left(2^{-2 j^{*} \min \left\{\tilde{s}_{i}\right\}}+T^{-1 / 2} 2^{-j^{*} \min \left\{m_{i}-1 / 2-1 /\left(2 p_{i}\right)\right\}}\right)\right)=O\left(T^{1 / 2}\right)
\end{aligned}
$$

Pela equação (A.10) de Dahlhaus et al. (1999) temos que

$$
\left\|A_{2}\right\|_{\infty}=O\left(2^{j^{*} / 2}\left\|\sum_{j \geq j^{*}} \sum_{k \in \mathcal{I}_{j}} \beta_{j k}^{\left(\omega_{0}\right)} \psi_{j k}\left(\frac{t}{T}\right)\right\|_{\infty}\right)=O\left(2^{j^{*} / 2}\right) .
$$

Usando o Lema A.2.1, temos que

$$
\left|c u m_{n}\left(\mathbf{\Upsilon}^{\prime} \mathbf{S}\right)_{u}\right| \leq C^{n}(n !)^{(2+2 \gamma)} T\left(2^{j^{*} / 2}\right)^{n-2} \quad \text { para } \quad n \geq 2
$$

o que implica, juntamente com $\mathbb{E}\left(\Upsilon^{\prime} \mathbf{S}\right)_{u}=\max \left(O\left(\widetilde{A_{1}}\right), O\left(\widetilde{A_{2}}\right)\right)=O\left(T^{1 / 2}\right)$ que

$$
\mathbb{E}\left\|\Upsilon^{\prime} \mathbf{S}\right\|^{s}=O\left(2^{j^{*} s / 2} T^{s / 2}\right)
$$

Como $\boldsymbol{\zeta}-\hat{\boldsymbol{\zeta}}=\left(\boldsymbol{\Upsilon}^{\prime} \boldsymbol{\Upsilon}\right)^{-1}\left(\boldsymbol{\Upsilon}^{\prime} \mathbf{e}+\boldsymbol{\Upsilon}^{\prime} \mathbf{S}\right)$ temos 


$$
\begin{aligned}
\boldsymbol{E}\left|\beta_{j k}^{(i)}-\hat{\beta}_{j k}^{(i)}\right|^{2+\varrho_{1}}= & \boldsymbol{E}\left|\boldsymbol{\Gamma}_{i, j, k}^{\prime}(\boldsymbol{\zeta}-\hat{\boldsymbol{\zeta}})\right|^{2+\varrho_{1}} \\
\leq & \boldsymbol{E}\left(\left\|\left(\boldsymbol{\Upsilon}^{\prime} \boldsymbol{\Upsilon}\right)^{-1}\right\|_{2}\left(\left\|\boldsymbol{\Upsilon}^{\prime} \mathbf{e}\right\|_{2}+\left\|\boldsymbol{\Upsilon}^{\prime} \mathbf{S}\right\|_{2}\right)\right)^{2+\varrho_{1}} \\
\leq & \left(\boldsymbol{E}\left\|\left(\boldsymbol{\Upsilon}^{\prime} \boldsymbol{\Upsilon}\right)^{-1}\right\|^{2+\varrho}\right)^{\frac{\left(2+e_{1}\right)}{2+\varrho}} \\
& \left(\boldsymbol{E}\left(\left\|\boldsymbol{\Upsilon}^{\prime} \mathbf{e}\right\|+\left\|\boldsymbol{\Upsilon}^{\prime} \mathbf{S}\right\|\right)^{\frac{\left(2+\varrho_{1}\right)\left(2+\varrho^{\prime}\right)}{\varrho^{-\varrho_{1}}}}\right)^{1-\frac{2+e_{1}}{2+e}} \\
= & O\left(T^{-\left(2+\varrho_{1}\right)}\right) O\left(\left(2^{j^{*} / 2} T^{1 / 2}\right)^{2+\varrho_{1}}\right) \\
= & O\left(\left(2^{j^{*} / 2} T^{-1 / 2}\right)^{2+\varrho_{1}}\right)=O(1) .
\end{aligned}
$$

O que completa a prova.

Ao generalizarmos para quaisquer $r, s$ e $b$ positivos, teremos que $\left\{e_{t}\right\}$ é um $M A(r)$ e as matrizes construídas nesta prova terão a mesma estrutura, porém com mais blocos. Assim esse lema será válido, bem como o Lema A.3.3. Para o segundo estágio, genericamente, temos que $\left\{n_{t}\right\}$ é um processo $M A(2 r)$.

Os lemas apresentados a seguir são de Dahlhaus et al. (1999).

Lema A.3.3: Seja $j^{*}=j^{*}(T) \rightarrow \infty, j^{*}=o(T)$ e $\Delta^{*}=\max \{r, s+b\}$.

$$
\begin{aligned}
& \text { (i) }\left\|\left(\mathbb{E} \mathbf{\Upsilon}^{\prime} \mathbf{\Upsilon}\right)^{-1}\right\|_{\infty}=O\left(T^{-1}\right) ; \\
& \text { (ii) }\left\|\operatorname{cov}\left(\mathbf{\Upsilon}^{\prime} \epsilon\right)-\left(\left\{T \int \phi_{j^{*}, u}(s) \phi_{j^{*}, v}(s) \sigma^{2}(s) c(s, k-l) d s\right\}\right)_{\Delta^{*}(u-1)+k, \Delta^{*}(v-1)+l}\right\|_{\infty}= \\
& o(T) ; \\
& \text { (iii) }\left\|\left(\mathbf{\Upsilon}^{\prime} \mathbf{\Upsilon}\right)^{-1}-\left(\mathbb{E} \mathbf{\Upsilon}^{\prime} \mathbf{\Upsilon}\right)^{-1}\right\|_{\infty}=O_{p}\left(2^{j^{*} / 2} T^{-3 / 2} \sqrt{\log (T)}\right) ; \\
& \text { (iv) }\left\|\mathbf{\Upsilon}^{\prime} \epsilon\right\|_{2}^{2}=O_{p}\left(2^{j^{*}} T \log (T)\right) ; \\
& \text { (v) }\left\|\mathbf{\Upsilon}^{\prime} \mathbf{S}\right\|_{2}^{2}=O_{p}\left(T^{2}\left(2^{-2 j^{*} \min \left\{\tilde{s}_{i}\right\}}+T^{-1} 2^{-j^{*} \min \left\{2 m_{i}-1-1 / p_{i}\right\}}\right) \log (T)\right) .
\end{aligned}
$$


Prova. (i) Seja $M=\operatorname{Tdiag}\left[M_{1}, M_{2}, \cdots, M_{\Delta}\right]$ em que $M_{u}=\Sigma_{t}$ para qualquer $t$ com $t / T \in$ suporte $\left(\phi_{j^{*}, u}\right)$.

Se $M^{-1}=T^{-1} \operatorname{diag}\left[M_{1}^{-1}, M_{2}^{-1}, \cdots, M_{\Delta}^{-1}\right]$, dos itens $(i)$ e $(i i)$ do Lema A.3.1, sabemos que

$$
\left\|M^{-1}\right\|_{\infty}=O\left(T^{-1}\right)
$$

$\operatorname{com} j^{*}=j^{*}(T) \rightarrow \infty$ e $j^{*}=o(T)$

O elemento $(\max \{r, s+b\})(u-1)+k,(\max \{r, s+b\})(v-1)+l \operatorname{de}\left(\boldsymbol{E} \boldsymbol{\Upsilon}^{\prime} \mathbf{\Upsilon}-M\right)$ será:

$$
\begin{aligned}
\left(\boldsymbol{E} \Upsilon^{\prime} \boldsymbol{\Upsilon}-M\right)_{k l}= & \sum_{t=2}^{T} \phi_{j^{*}, u}\left(\frac{t}{T}\right) \phi_{j^{*}, v}\left(\frac{t}{T}\right)\left[\left(\Sigma_{t}\right)_{k l}-\left(M_{u}\right)_{k l}\right]+ \\
& +\left[\sum_{t=2}^{T} \phi_{j^{*}, u}\left(\frac{t}{T}\right) \phi_{j^{*}, v}\left(\frac{t}{T}\right)-T \delta_{u v}\right]\left(M_{u}\right)_{k l}=o(T)
\end{aligned}
$$

vale uniformemente em $u, v, k$ e $l$. Como $\phi_{j^{*}, u}$ e $\phi_{j^{*}, v}$ têm suporte disjuntos para $|u-v| \geq C$, temos que $\left(\boldsymbol{E} \boldsymbol{\Upsilon}^{\prime} \boldsymbol{\Upsilon}\right)_{k l}=0$ para $|k-l| \geq C(\max \{r, s+b\})$.

De (A.4) temos que

$$
\left\|E \Upsilon^{\prime} \Upsilon-M\right\|_{\infty}=o(T)
$$

De (A.4) e (A.5) sabemos que existe um $T_{0}$ tal que $\left\|M^{-1 / 2}\left(\mathbb{E} \Upsilon^{\prime} \Upsilon-M\right) M^{-1 / 2}\right\|_{\infty} \leq$ $C<1$ para todo $T \geq T_{0}$.

Pela decomposição espectral da matriz $\left(I+M^{-1 / 2}\left(\mathbb{E} \boldsymbol{\Upsilon}^{\prime} \Upsilon-M\right) M^{-1 / 2}\right)$ vale a fórmula de inversão

$$
\begin{aligned}
\left(\Upsilon^{\prime} \Upsilon\right)^{-1} & =\left[M^{1 / 2}\left(I+M^{-1 / 2}\left(E \Upsilon^{\prime} \Upsilon-M\right) M^{-1 / 2}\right) M^{1 / 2}\right]^{-1} \\
& =M^{-1 / 2}\left[I+\sum_{s=1}^{\infty}(-1)^{s}\left(M^{-1 / 2}\left(\mathbb{E} \Upsilon^{\prime} \Upsilon-M\right) M^{-1 / 2}\right)^{s}\right] M^{-1 / 2}
\end{aligned}
$$


o que completa a prova de $(i)$.

(ii) Como $\boldsymbol{E}\left(\boldsymbol{\Upsilon}^{\prime} \boldsymbol{\epsilon}\right)=0$ temos que $\operatorname{cov}\left(\boldsymbol{\Upsilon}^{\prime} \boldsymbol{\epsilon}\right)=\boldsymbol{E}\left(\boldsymbol{\Upsilon}^{\prime} \boldsymbol{\epsilon} \boldsymbol{\epsilon}^{\prime} \boldsymbol{\Upsilon}\right)$

A matriz $A=\boldsymbol{\Upsilon}^{\prime} \boldsymbol{\epsilon} \boldsymbol{\epsilon}^{\prime} \Upsilon \mathbf{~ e ́ ~ s i m e ́ t r i c a ~ e ~ f o r m a d a ~ p o r ~}(r+s+1)^{2}$ blocos, cada bloco com dimensão $\left(2^{j^{*}}\right) \times\left(2^{j^{*}}\right)$. Considerando $r=1$ e $s=0$, o primeiro bloco (diagonal) terá como componentes os elementos $(u, v), u, v=1, \cdots, 2^{j^{*}}$.

$$
\begin{aligned}
A_{u, v} & =\sum_{s=2}^{T} \phi_{j^{*}, u}(s / T) Y_{s-1, T} \epsilon_{s} \sum_{t=2}^{T} \phi_{j^{*}, u}(t / T) Y_{t-1, T} \epsilon_{t} \\
& =\sum_{s=2}^{T} \sum_{t=2}^{T} \phi_{j^{*}, u}(s / T) \phi_{j^{*}, u}(t / T) Y_{s-1, T} Y_{t-1, T} \epsilon_{s} \epsilon_{t}
\end{aligned}
$$

ao calcularmos a esperança temos:

$$
\begin{aligned}
\boldsymbol{E}\left(A_{u, v}\right) & =\sum_{s=2}^{T} \sum_{t=2}^{T} \phi_{j^{*}, u}(s / T) \phi_{j^{*}, u}(t / T) \boldsymbol{E}\left(Y_{s-1, T} Y_{t-1, T}\right) \boldsymbol{E}\left(\epsilon_{s} \epsilon_{t}\right) \\
& =\sum_{s=2}^{T} \phi_{j^{*}, u}(s / T) \phi_{j^{*}, u}(s / T) \mathbb{E}\left(Y_{s-1, T}^{2}\right) \boldsymbol{E}\left(\epsilon_{s}^{2}\right) \\
& =T \int \phi_{j^{*}, u}(s / T) \phi_{j^{*}, u}(s / T) \sigma^{2} c_{y y}(s, 0)+o(T)
\end{aligned}
$$

Esse resultado foi obtido de maneira similar a (A.5).

Para o segundo bloco da diagonal temos o resultado análogo,

$$
\boldsymbol{E}\left(A_{u, v}\right)=T \int \phi_{j^{*}, u}(s / T) \phi_{j^{*}, u}(s / T) \sigma^{2} c_{x x}(s, 0)+o(T)
$$

Para os dois blocos, fora da diagonal, que são simétricos, temos:

$$
\mathbb{E}\left(A_{u, v}\right)=T \int \phi_{j^{*}, u}(s / T) \phi_{j^{*}, u}(s / T) \sigma^{2} c_{x y}(s, 1)+o(T)
$$

Generalizando para $r$ e $s$ quaisquer, teremos em cada bloco diferentes valores de $l=k-s$ e as covariâncias serão $c_{y y}(s, l), c_{x x}(s, l)$ e $c_{x y}(s, l)$, as mesmas apresentadas na prova do Lema A.3.1. 
(iii) A matriz $\Upsilon^{\prime} \Upsilon$ é uma matriz composta por $(r+s+1) \times(r+s+1)$ matrizes quadradas, cada submatriz é de dimensão $(T-\max \{r, s+b\}) \times(T-\max \{r, s+b\})$. Escreveremos aqui a forma dessa matriz para o caso mais simples, isto é, $r=1$, $s=b=0$ :

$$
\Upsilon^{\prime} \Upsilon=\left[\begin{array}{ll}
U_{11} & U_{12} \\
U_{21} & U_{22}
\end{array}\right]
$$

em que

$$
\begin{aligned}
\left(U_{11}\right)_{u v} & =\sum_{t=2}^{T} \phi_{j^{*}, u}\left(\frac{t}{T}\right) \phi_{j^{*}, v}\left(\frac{t}{T}\right) Y_{t-1, T}^{2} \\
\left(U_{12}\right)_{u v} & =\sum_{t=2}^{T} \phi_{j^{*}, u}\left(\frac{t}{T}\right) \phi_{j^{*}, v}\left(\frac{t}{T}\right) Y_{t-1, T} X_{t, T} \\
\left(U_{21}\right)_{u v} & =\sum_{t=2}^{T} \phi_{j^{*}, u}\left(\frac{t}{T}\right) \phi_{j^{*}, v}\left(\frac{t}{T}\right) X_{t, T} Y_{t-1, T} \\
\left(U_{22}\right)_{u v} & =\sum_{t=2}^{T} \phi_{j^{*}, u}\left(\frac{t}{T}\right) \phi_{j^{*}, v}\left(\frac{t}{T}\right) X_{t, T}^{2}
\end{aligned}
$$

Substituindo os processos $\left\{X_{t, T}\right\}$ e $\left\{Y_{t, T}\right\}$ por sua representação $M A(\infty)$ dadas em (A.1) e (A.2), respectivamente temos

$$
\begin{aligned}
\left(U_{11}\right)_{u v}= & \sum_{l=0}^{\infty} \sum_{s=0}^{\infty} \sum_{t=2}^{T} \phi_{j^{*}, u}\left(\frac{t}{T}\right) \phi_{j^{*}, v}\left(\frac{t}{T}\right) \gamma_{t-1, T}^{Y}(s) a_{t-1-s} \gamma_{t-1, T}^{Y}(l) a_{t-1-l}+ \\
& +2 \sum_{s=0}^{\infty} \sum_{t=2}^{T} \phi_{j^{*}, u}\left(\frac{t}{T}\right) \phi_{j^{*}, v}\left(\frac{t}{T}\right) \gamma_{t-1, T}^{Y}(s) a_{t-1-s} \epsilon_{t-1}+ \\
& +\sum_{t=2}^{T} \phi_{j^{*}, u}\left(\frac{t}{T}\right) \phi_{j^{*}, v}\left(\frac{t}{T}\right) \epsilon_{t-1, T}^{2} \\
\left(U_{12}\right)_{u v}= & \sum_{l=0}^{\infty} \sum_{s=0}^{\infty} \sum_{t=2}^{T} \phi_{j^{*}, u}\left(\frac{t}{T}\right) \phi_{j^{*}, v}\left(\frac{t}{T}\right) \gamma_{t-1, T}^{Y}(s) \gamma_{t, T}^{X}(l) a_{t-1-s} a_{t-1-l}+ \\
& +\sum_{s=0}^{\infty} \sum_{t=2}^{T} \phi_{j^{*}, u}\left(\frac{t}{T}\right) \phi_{j^{*}, v}\left(\frac{t}{T}\right) \gamma_{t, T}^{X}(s) a_{t-s} \epsilon_{t-1},
\end{aligned}
$$




$$
\begin{aligned}
\left(U_{22}\right)_{u v}= & \sum_{l=0}^{\infty} \sum_{s=0}^{\infty} \sum_{t=2}^{T} \phi_{j^{*}, u}\left(\frac{t}{T}\right) \phi_{j^{*}, v}\left(\frac{t}{T}\right) \gamma_{t, T}^{X}(s) \gamma_{t, T}^{X}(l) a_{t-s} a_{t-l}+ \\
& +\sum_{s=0}^{\infty} \sum_{t=2}^{T} \phi_{j^{*}, u}\left(\frac{t}{T}\right) \phi_{j^{*}, v}\left(\frac{t}{T}\right) \gamma_{t, T}^{X}(s) a_{t-s} \epsilon_{t-1} .
\end{aligned}
$$

A submatriz $U_{12}$ é a transposta da submatriz $U_{21}$. Desta forma, vemos que $\Upsilon^{\prime} \Upsilon$ pode ser escrita como uma combinação de formas quadráticas que resulta na forma $x^{\prime} A x$ e para cada submatriz de $A$ temos:

$$
\|A\|_{\infty}=O\left(2^{j^{*}}\right), \quad \tilde{A}=O(T) .
$$

Pelo Lema A.2.1, temos que

$$
\begin{aligned}
\left|\operatorname{cum}_{n}\left(\Upsilon^{\prime} \Upsilon\right)_{u, v}\right| & \leq C^{n}(n !)^{2+2 \gamma}\left(2^{j^{*}}\right)^{n-1} T \\
& \leq\left(\frac{n !}{2}\right)^{1+(1+2 \gamma)} \frac{H_{T}}{\bar{\Delta}_{T}^{n-2}}
\end{aligned}
$$

em que $H_{T} \sim 2^{j^{*}} T, \bar{\Delta}_{T} \sim 2^{-j^{*}}$. Daí pelo Lema A.2.3, obtemos que

$$
P\left(\left|\left(\Upsilon^{\prime} \Upsilon\right)_{u, v}-\left(\mathbb{E} \Upsilon^{\prime} \Upsilon\right)_{u, v}\right| \geq x\right) \leq \exp \left(-C \frac{x^{2}}{2^{j^{*} / 2} T}\right),
$$

para $0 \leq x \leq\left(H_{T}^{1+\gamma} \bar{\Delta}_{T}\right)^{1 /(1+2 \gamma)}$. Como $\left(H_{T}^{1+\gamma /(1+2 \gamma)} \bar{\Delta}_{T}\right) \sim 2^{j^{*} \gamma /(1+2 \gamma)} T^{(1+\gamma) /(1+2 \gamma)}$ $>>2^{j^{*} / 2} T^{1 / 2}$, obtemos que

$$
\left(\boldsymbol{\Upsilon}^{\prime} \boldsymbol{\Upsilon}\right)_{u, v}-\left(\boldsymbol{E} \boldsymbol{\Upsilon}^{\prime} \Upsilon\right)_{u, v}=O_{p}\left(2^{j^{*} / 2} T^{\mathbf{1} / 2} \sqrt{\log (T)}\right) .
$$

Como $\phi_{j^{*}, u}$ e $\phi_{j^{*}, v}$ têm suporte disjunto para $|u-v| \geq C$ obtemos imediatamente que

$$
\left\|\Upsilon^{\prime} \Upsilon-\mathbb{E} \Upsilon^{\prime} \Upsilon\right\|_{\infty}=O_{p}\left(2^{j^{*} / 2} T^{1 / 2} \sqrt{\log (T)}\right)
$$

que junto com o Lema A.3.3 (i), e com a fórmula de inversão

$$
\begin{aligned}
& \left\|\left(\boldsymbol{\Upsilon}^{\prime} \Upsilon\right)^{-1}-\left(\mathbb{E} \Upsilon^{\prime} \Upsilon\right)^{-1}\right\|_{\infty}= \\
= & \left\|\left(\mathbb{E} \Upsilon^{\prime} \Upsilon\right)^{-1}\right\|_{\infty} \sum_{s=1}^{\infty}\left(\left\|\Upsilon^{\prime} \Upsilon-\mathbb{E} \Upsilon^{\prime} \Upsilon\right\|_{\infty}\left\|\left(\mathbb{E} \Upsilon^{\prime} \Upsilon\right)^{-1}\right\|_{\infty}\right)^{s}= \\
= & O_{p}\left(2^{j^{*} / 2} T^{-3 / 2} \sqrt{\log (T)}\right),
\end{aligned}
$$


concluem a prova de $(i i i)$.

(iv) Segundo Dahlhaus et al. (1999) a prova deste item é feita de maneira similar.

(v) A prova desse Lema só envole as propriedades de ondaletas e está em Dahlhaus et al. (1999). 


\section{Apêndice B}

\section{Histogramas da Simulação}

Apresentamos aqui os histogramas feitos para alguns valores de $t, t=1,2, \cdots$, 2048 ao longo da curvas estimadas nos 2 casos simulados. Foram selecionados os pontos (e próximos a eles) nos quais algum dos parâmetros apresentou mudança ou comportamento atípico. Esses pontos são 10, 50, 300, 500, 512, 525, 1000, 1024, $1050,1520,1536,1550,1700,2000$ e 2035. Os valores conhecidos das funções para o ponto amostrado estão indicados em cada histograma.

As estimativas para $\delta_{1}(t)$, nos dois casos apresentados, são similares. Observamos a existência de viés no primeiro estágio que é corrigido no segundo estágio. Após a limiarização as estimativas são mais concentradas.

Nos histogramas apresentados para os dois casos simulados observamos que as estimativas para $\omega_{0}(t)$ são, em todos os passos, muitos semelhantes entre si. Após o uso da limiarização, as estimativas são mais concentradas, isto é, com menor variabilidade. Notamos a introdução de um viés na estimativa do segundo estágio de $\omega_{0}(t)$ após a limiarização. 
As estimativas para $\delta_{2}(t)$ são mais concentradas após a limiarização e não apresentam viéses, exceto para os extremos, em $t$, de $\tilde{\delta}_{2}^{(L)}(t)$. 


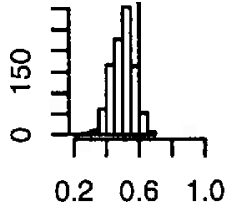

10
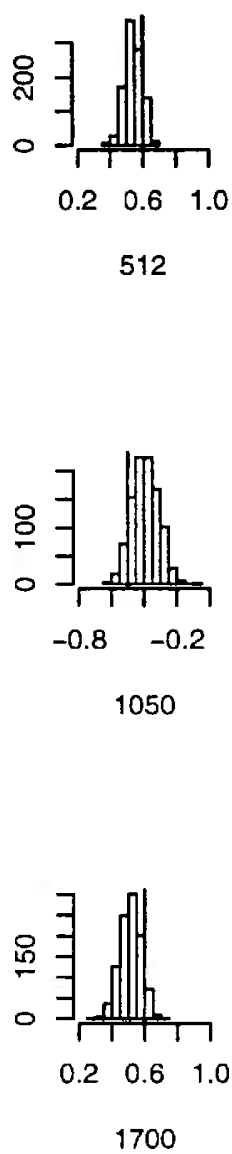

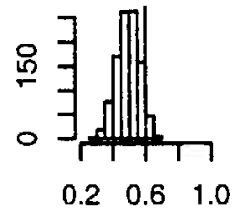

50
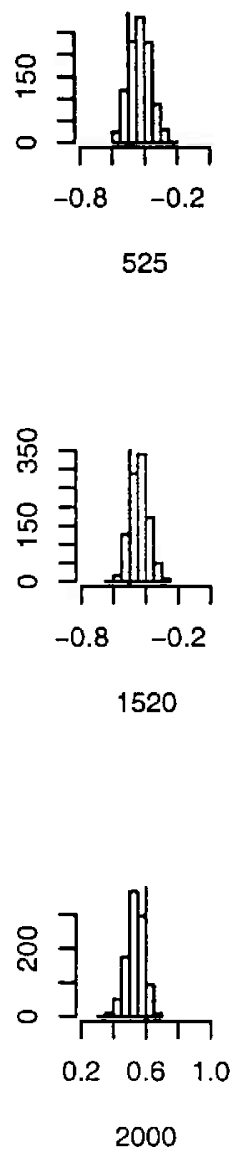
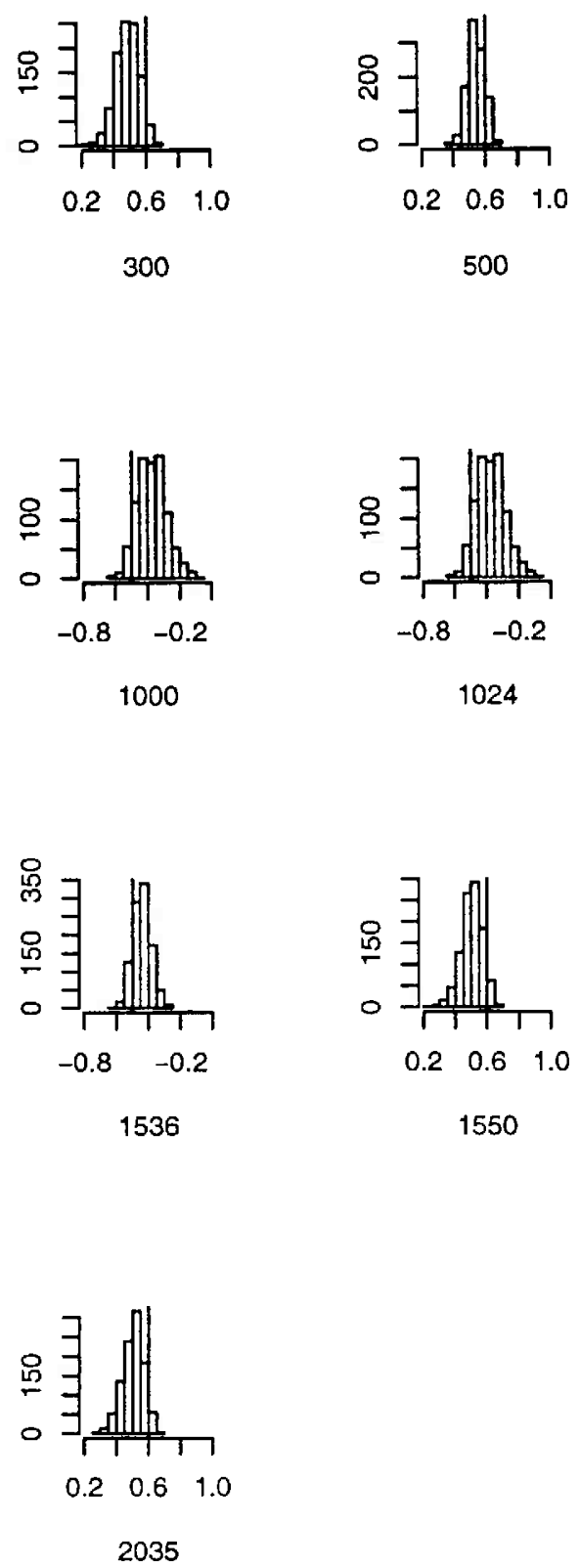

Figura B.1: Histogramas de $\hat{\delta}_{1}(t)$, Caso 1, nos diferentes valores de $t$. 

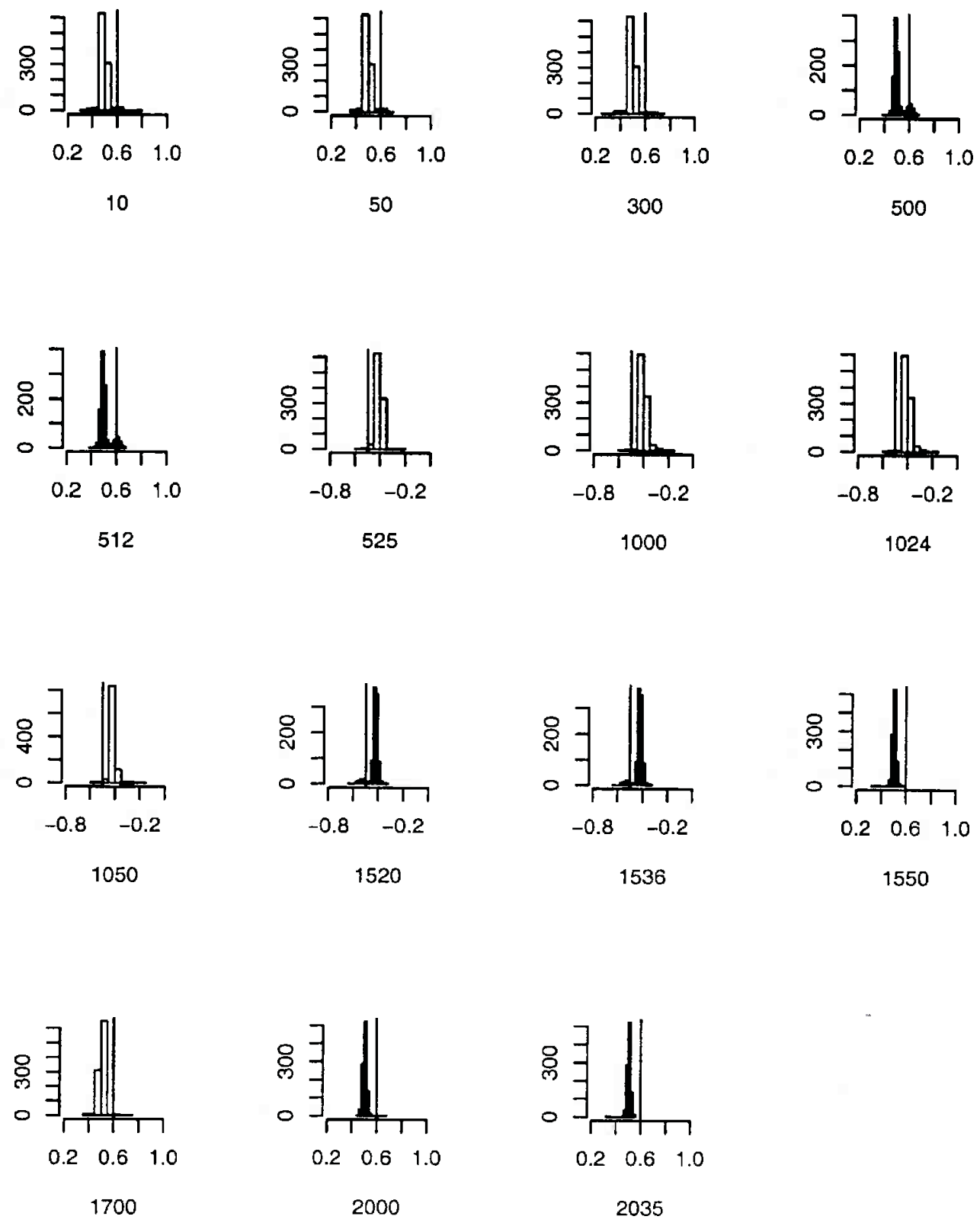

Figura B.2: Histogramas de $\hat{\delta}_{1}^{(L)}(t)$, Caso 1 , nos diferentes valores de $t$. 


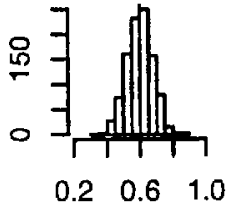

10
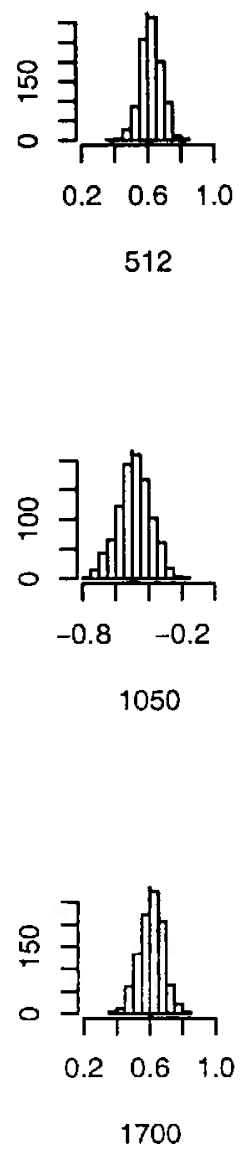

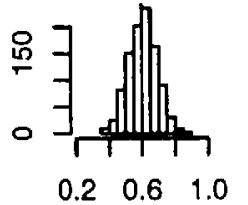

50
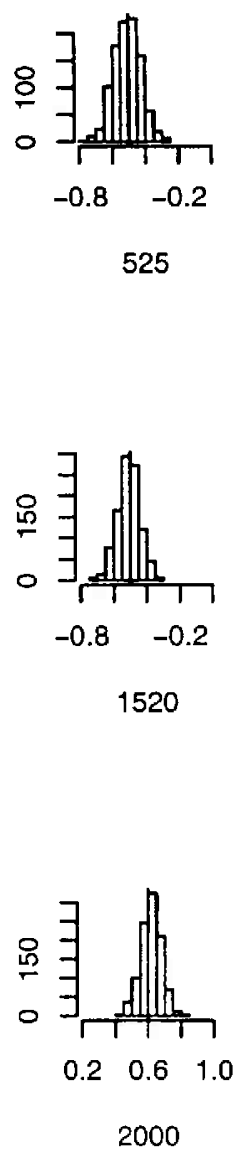
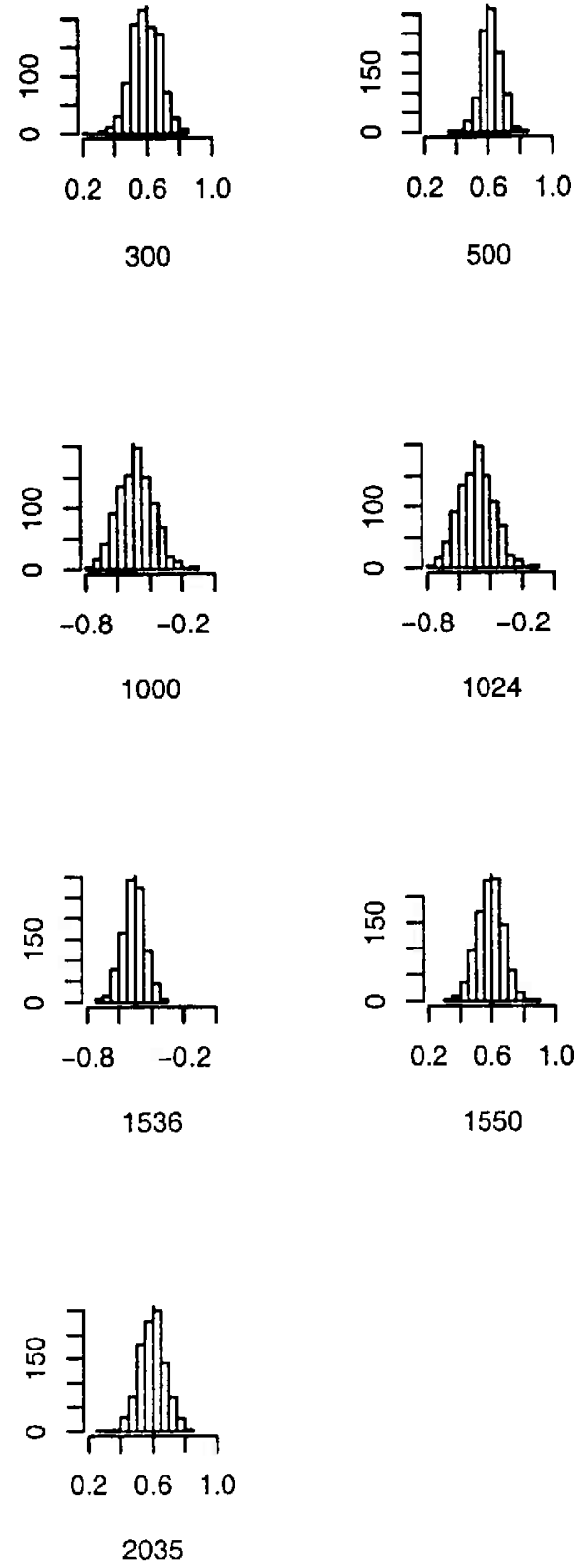

Figura B.3: Histogramas de $\tilde{\delta}_{1}(t)$, Caso 1 , nos diferentes valores de $t$. 

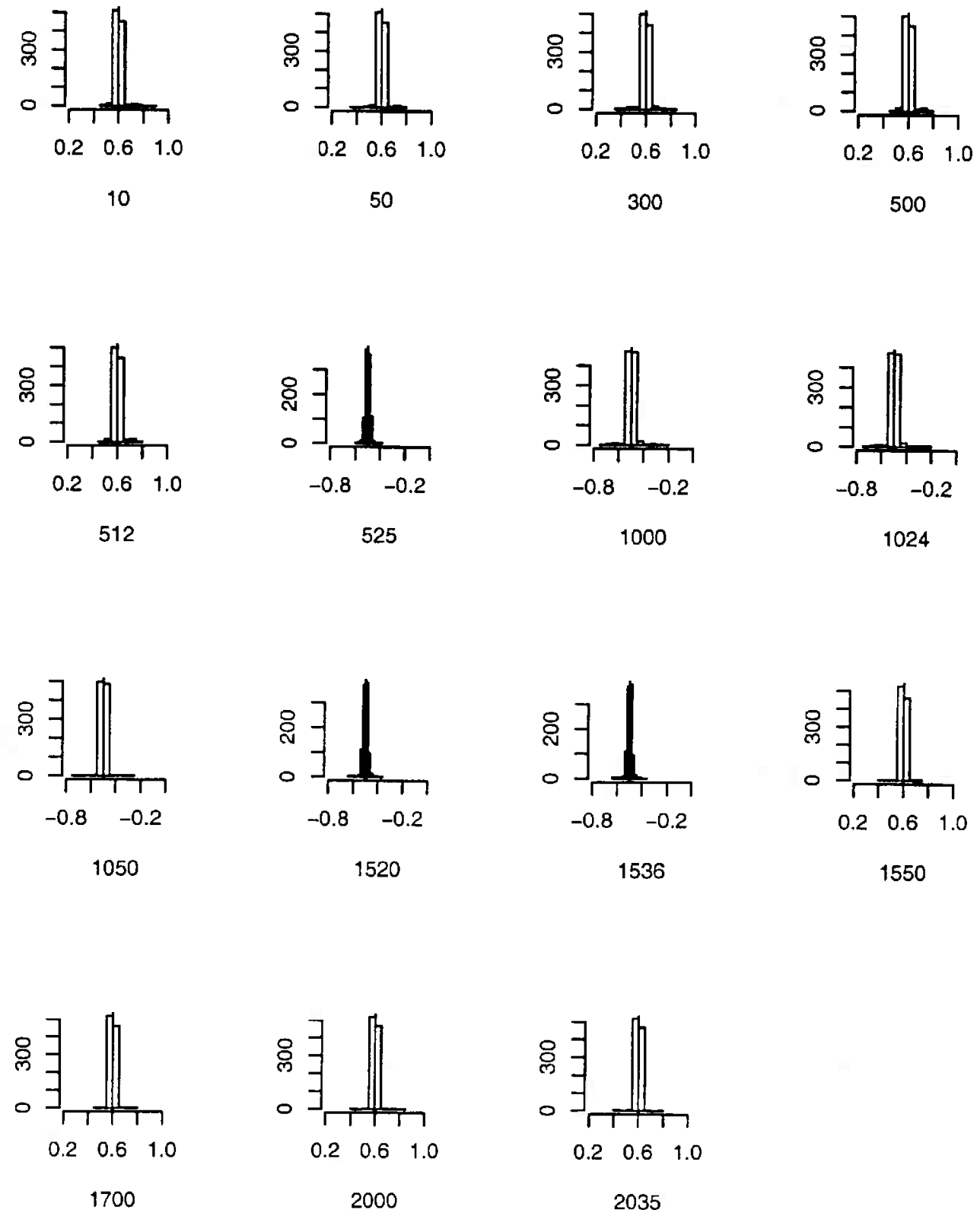

Figura B.4: Histogramas de $\tilde{\delta}_{1}^{(L)}(t)$, Caso 1 , nos diferentes valores de $t$. 


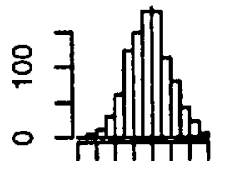

$1.2 \quad 2.0$

10
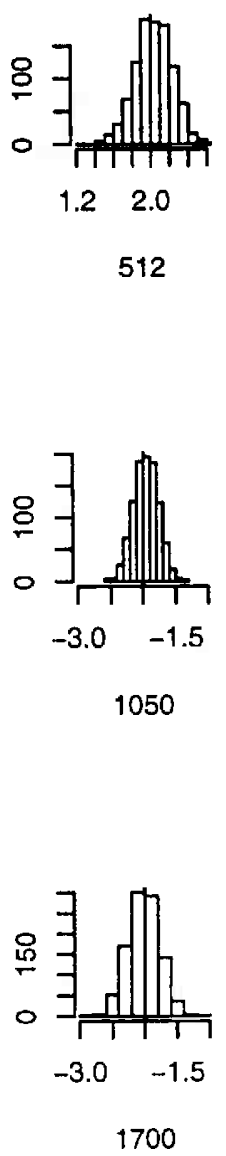
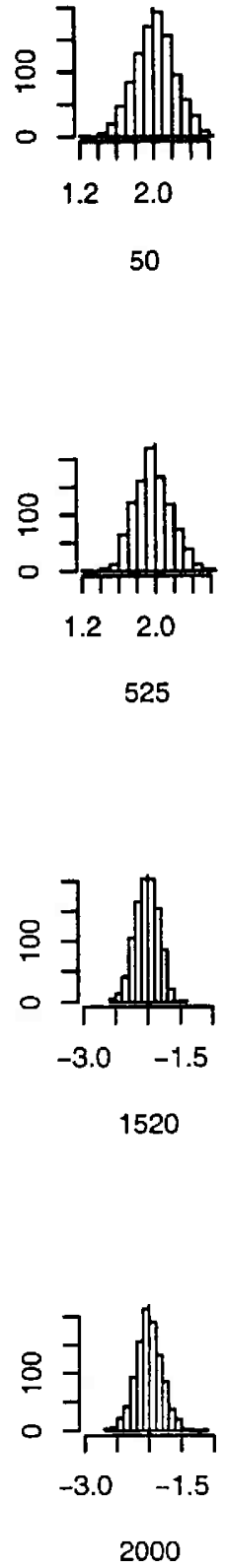
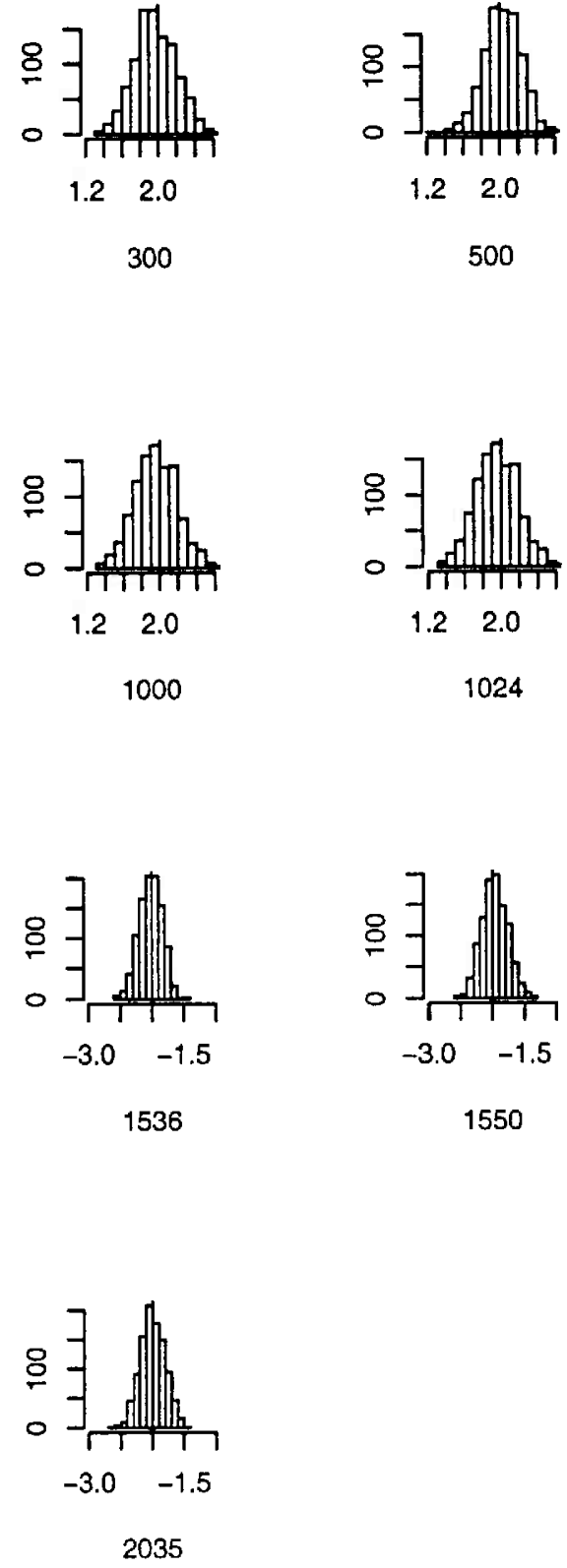

Figura B.5: Histogramas de $\hat{\omega}_{0}(t)$, Caso 1 , nos diferentes valores de $t$. 

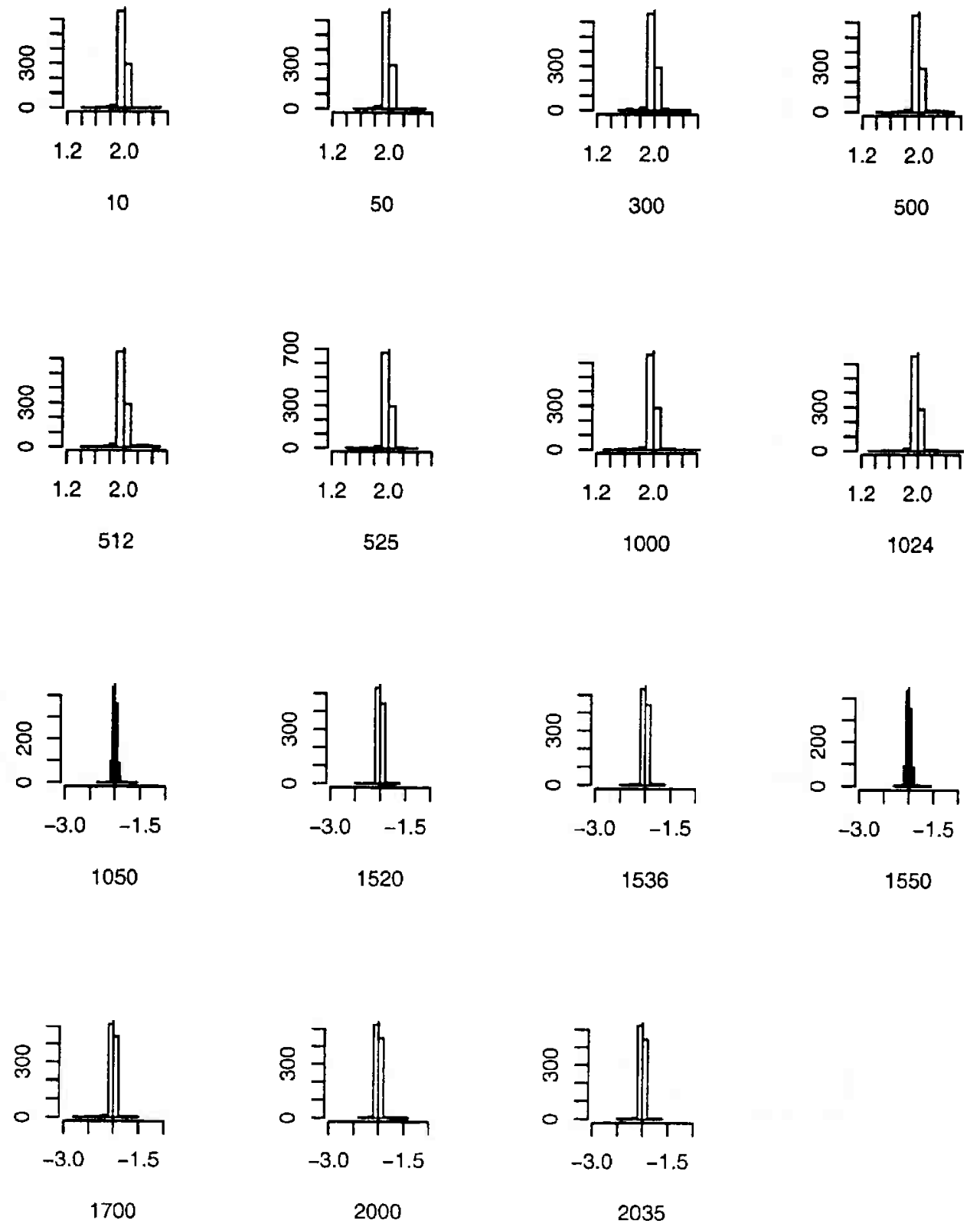

Figura B.6: Histogramas de $\hat{\omega}_{0}^{(L)}(t)$, Caso 1, nos diferentes valores de $t$. 


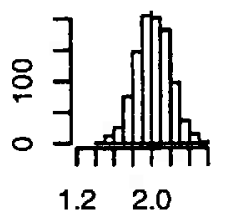

10
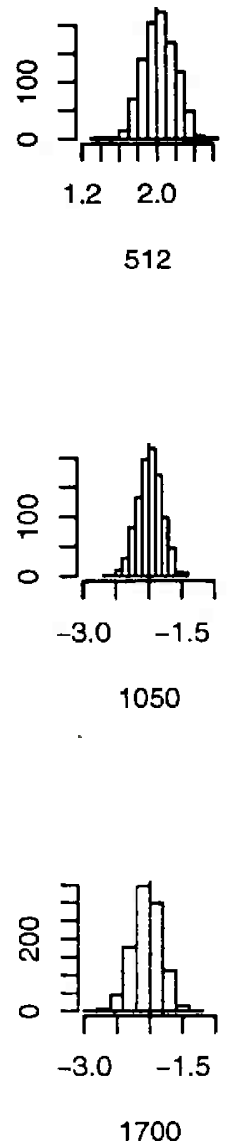

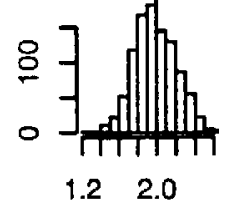

50
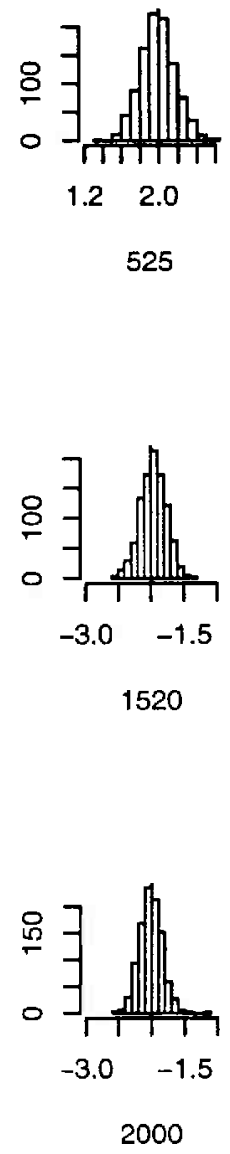

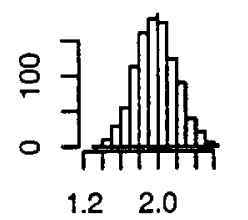

300
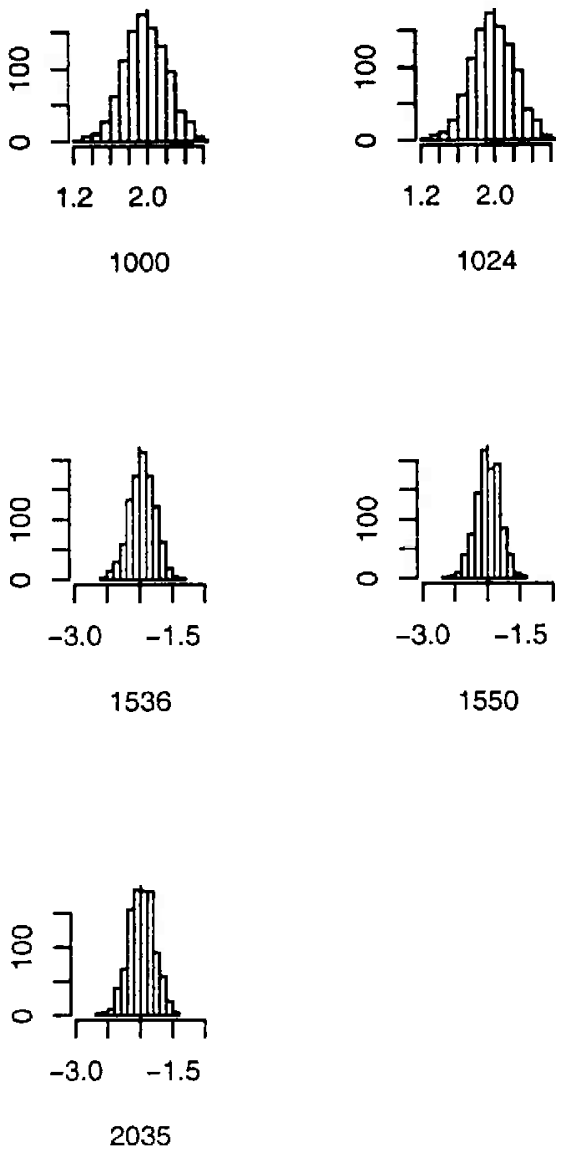

Figura B.7: Histogramas de $\tilde{\omega}_{0}(t)$, Caso 1 , nos diferentes valores de $t$. 

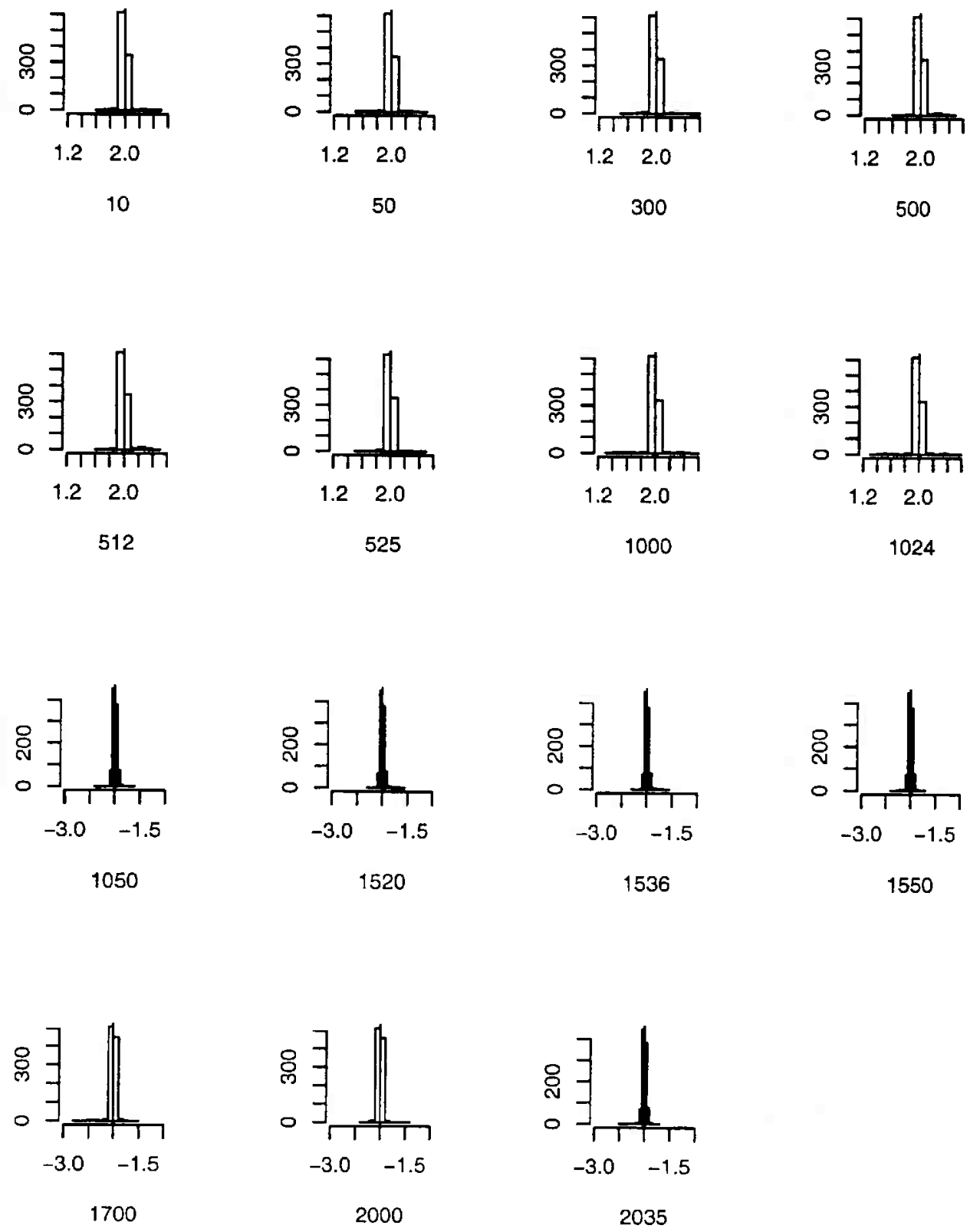

Figura B.8: Histogramas de $\tilde{\omega}_{0}^{(L)}(t)$, Caso 1, nos diferentes valores de $t$. 


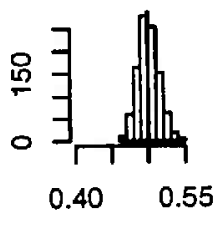

10
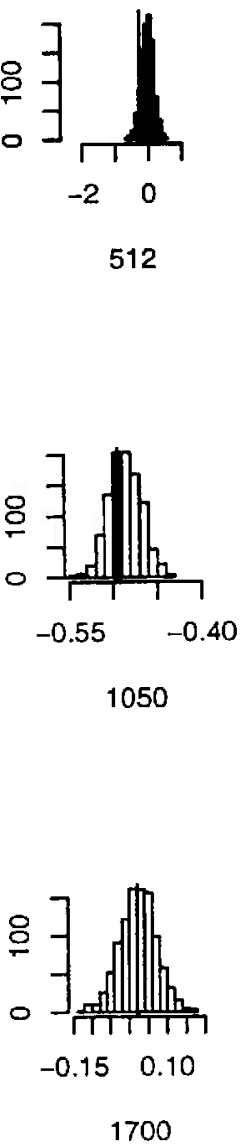

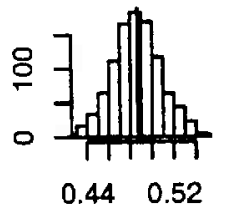

50
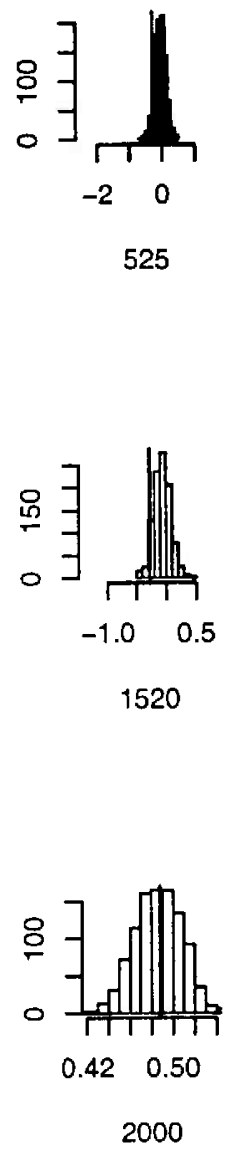
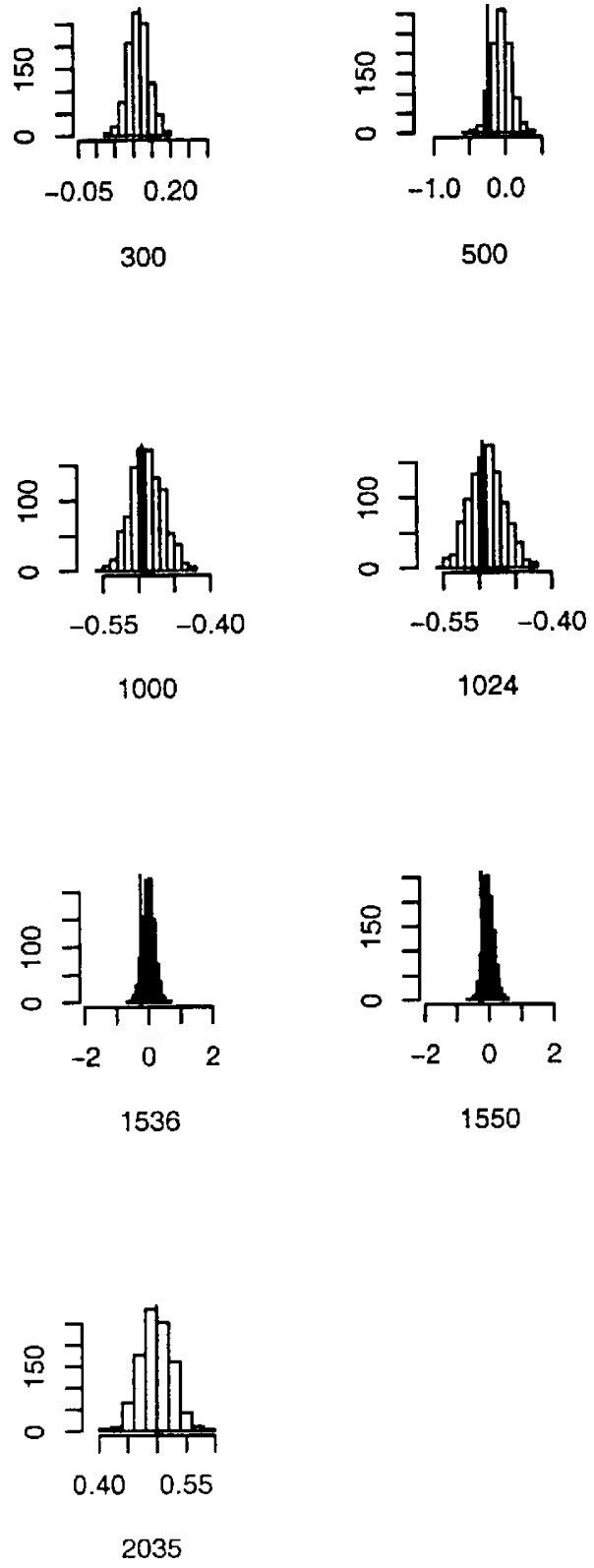

Figura B.9: Histogramas de $\hat{\delta}_{1}(t)$, Caso 2, nos diferentes valores de $t$. 

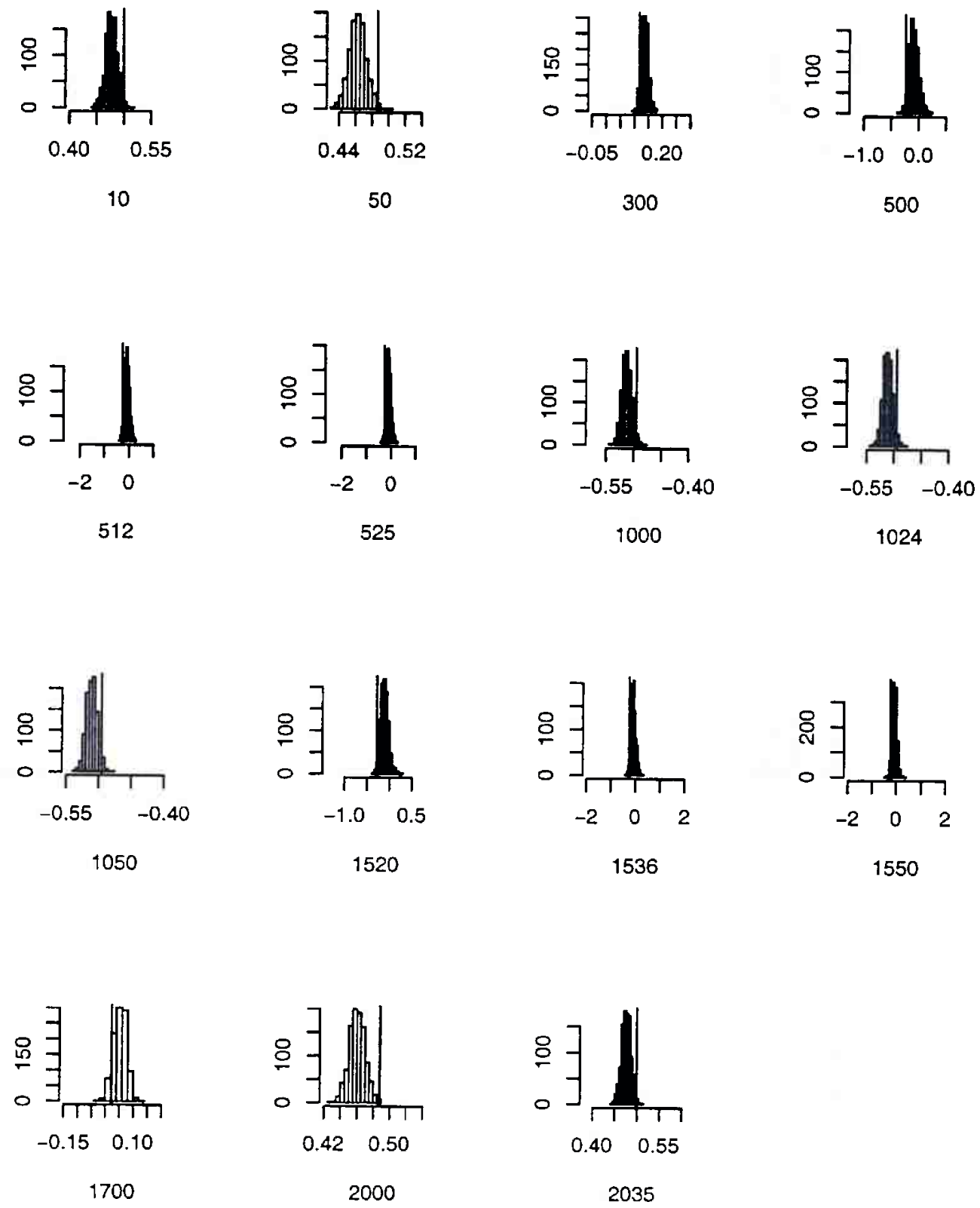

Figura B.10: Histogramas de $\hat{\delta}_{1}^{(L)}(t)$, Caso 2, nos diferentes valores de $t$. 

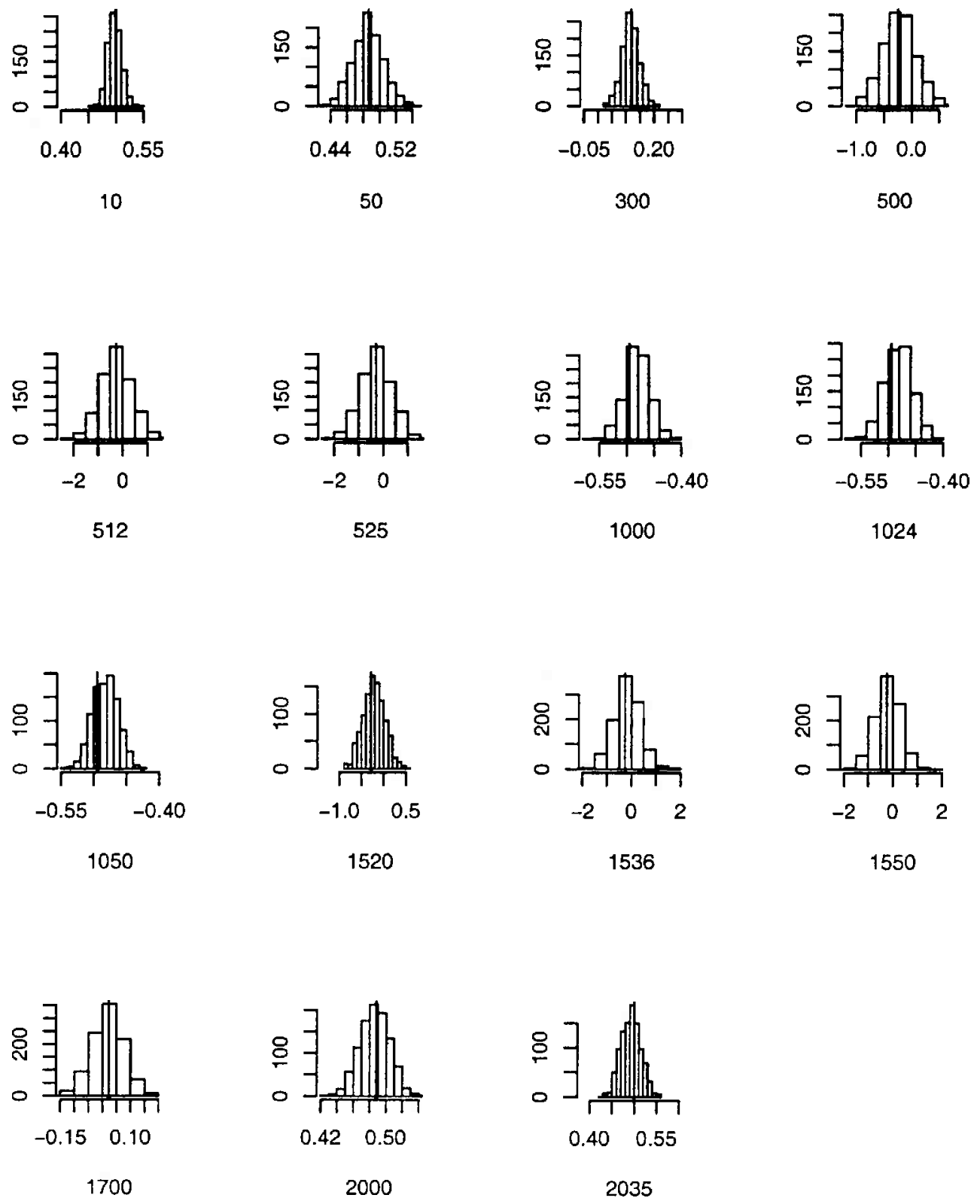

Figura B.11: Histogramas de $\tilde{\delta}_{1}(t)$, Caso 2, nos diferentes valores de $t$. 

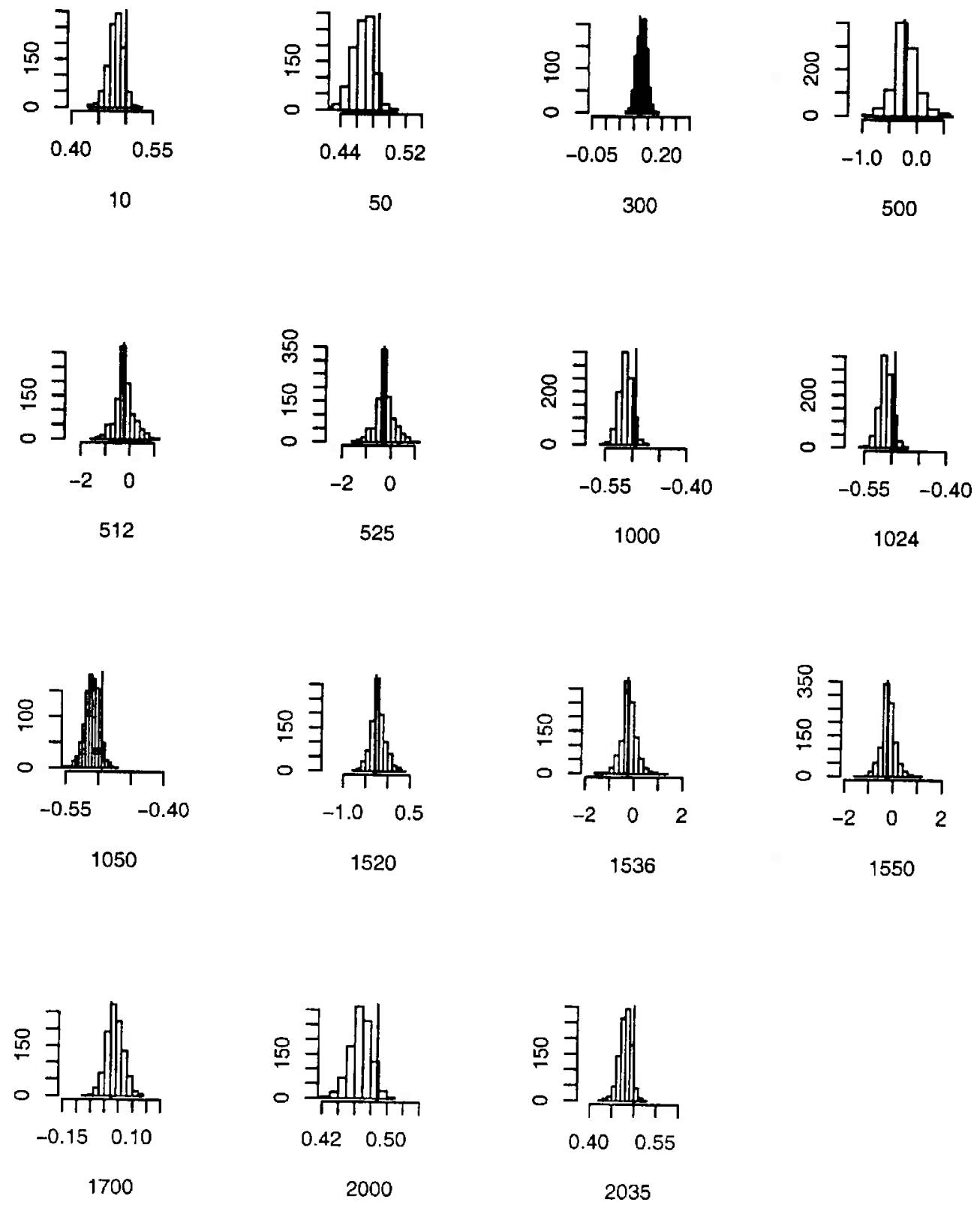

Figura B.12: Histogramas de $\tilde{\delta}_{1}^{(L)}(t)$, Caso 2, nos diferentes valores de $t$. 

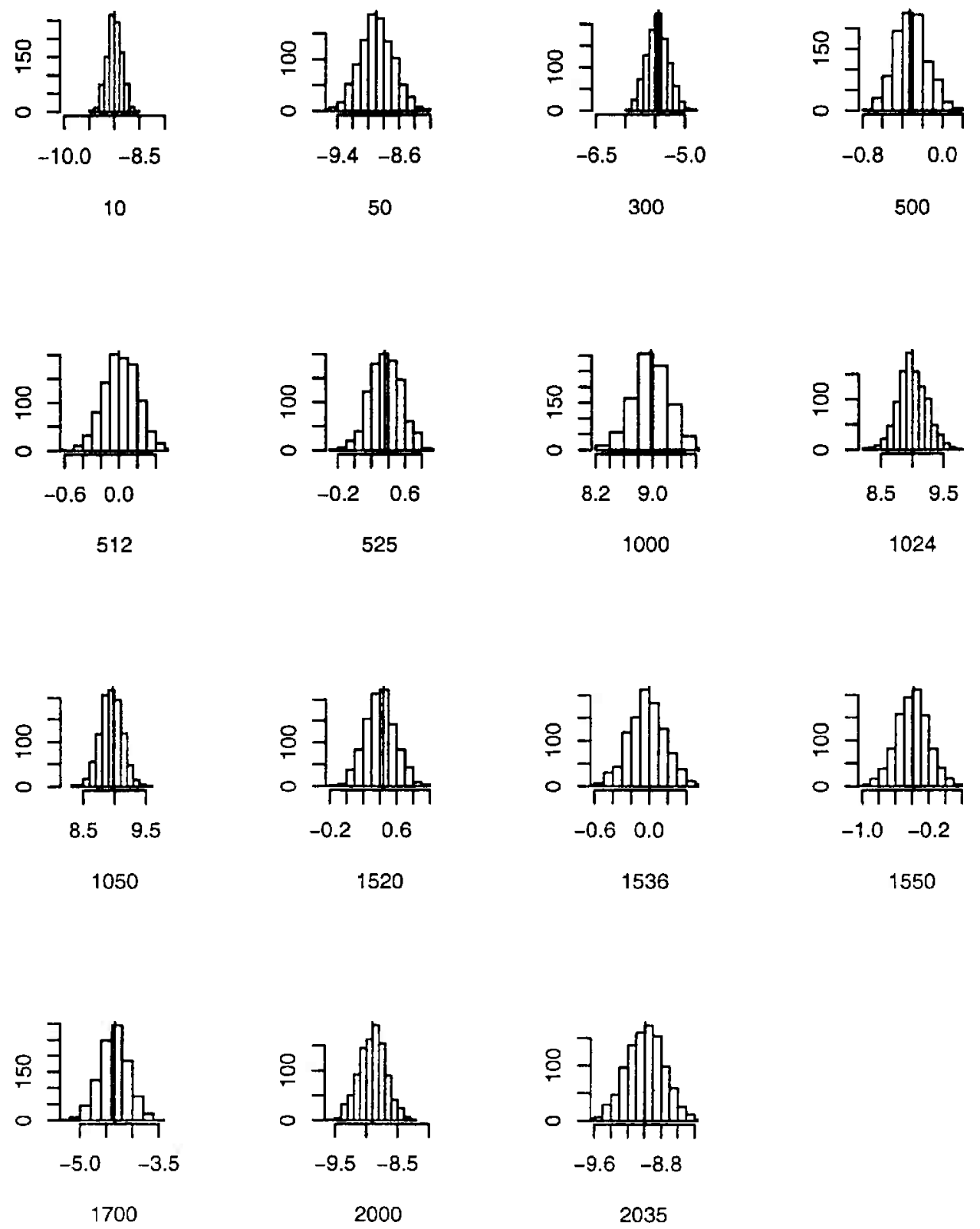

Figura B.13: Histogramas de $\hat{\omega}_{0}(t)$, Caso 2, nos diferentes valores de $t$. 

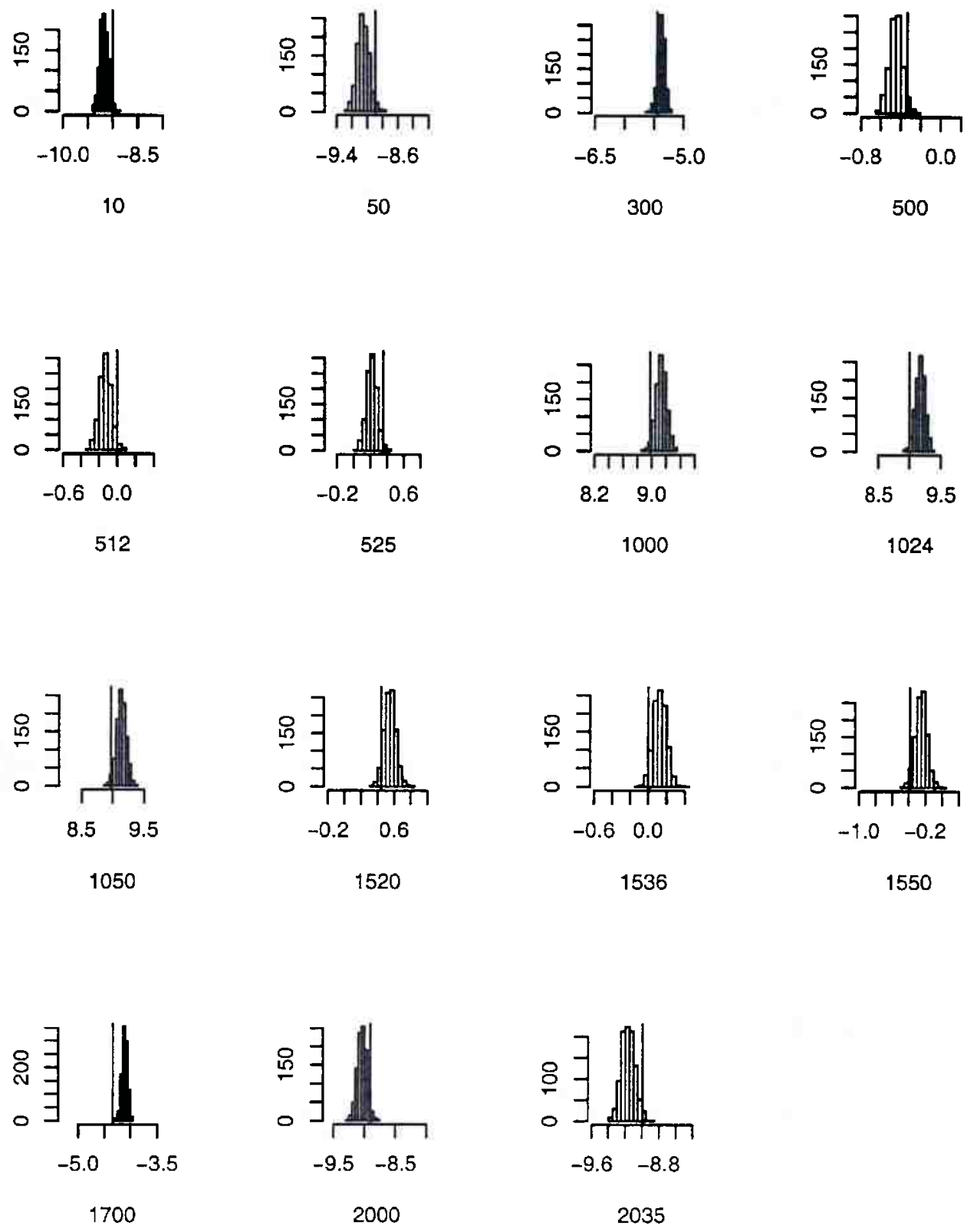

Figura B.14: Histogramas de $\hat{\omega}_{0}^{(L)}(t)$, Caso 2, nos diferentes valores de $t$. 

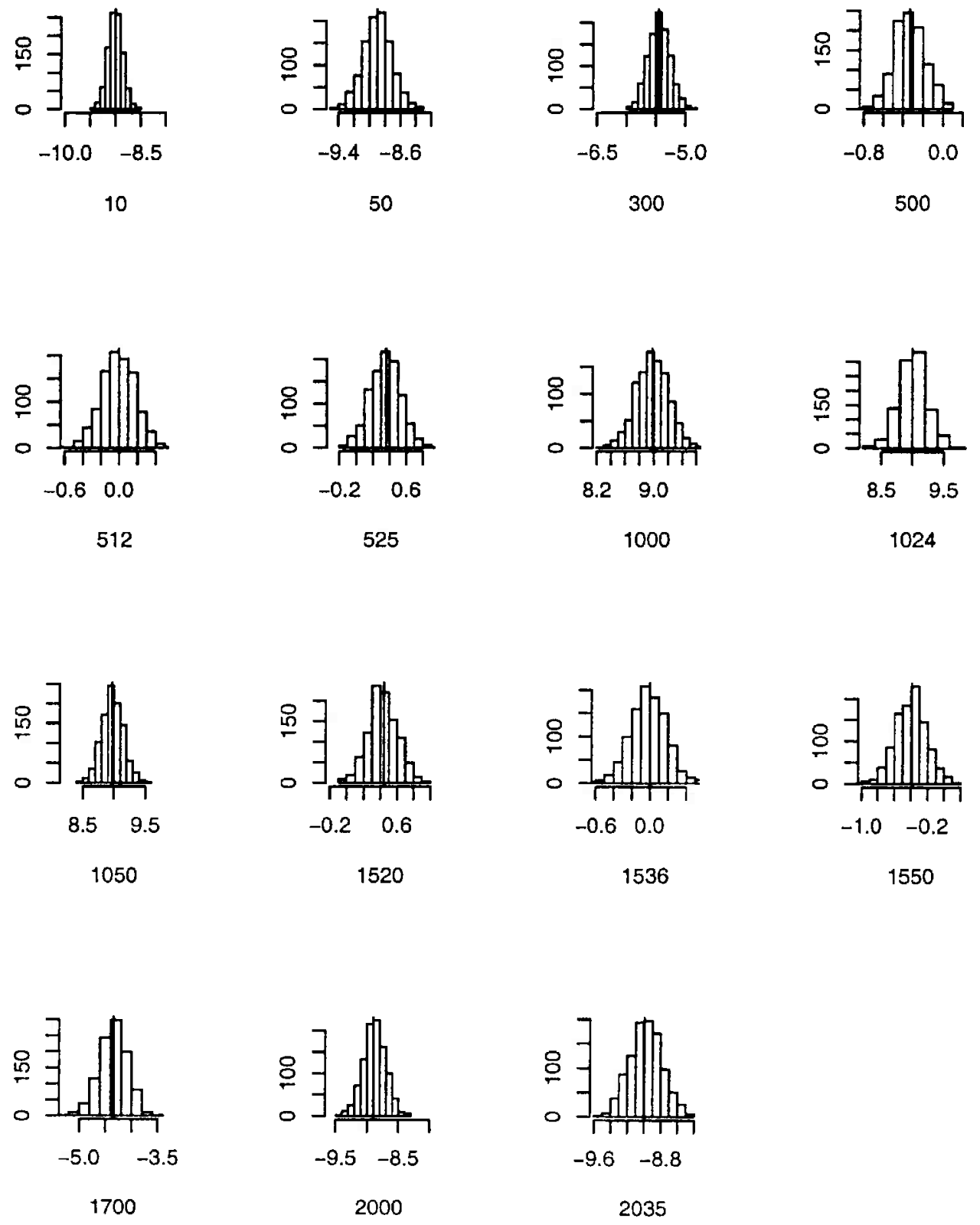

Figura B.15: Histogramas de $\tilde{\omega}_{0}(t)$, Caso 2, nos diferentes valores de $t$. 


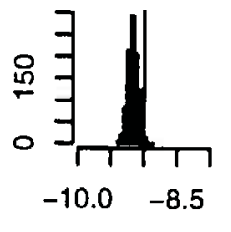

10

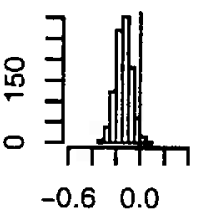

512

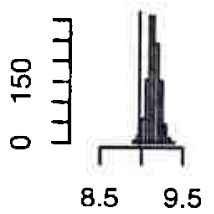

1050

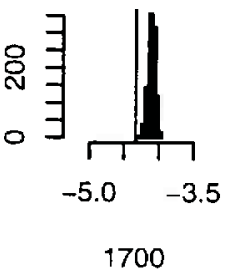

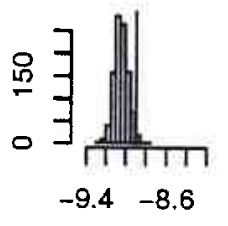

50

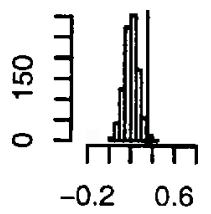

525
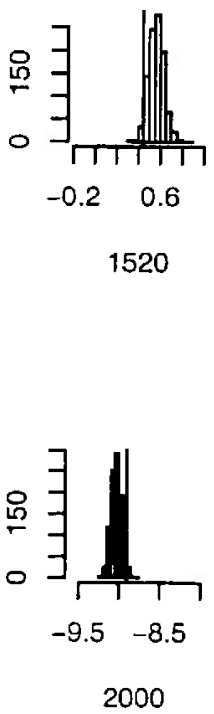
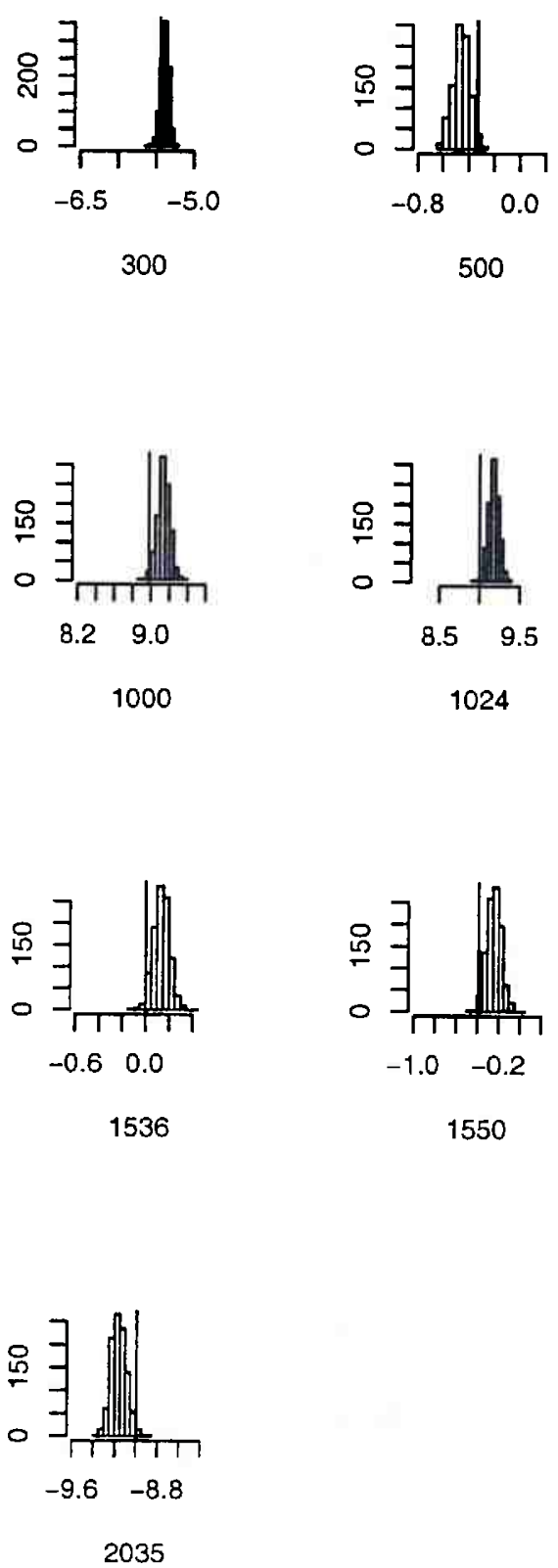

Figura B.16: Histogramas de $\tilde{\omega}_{0}^{(L)}(t)$, Caso 2, nos diferentes valores de $t$. 


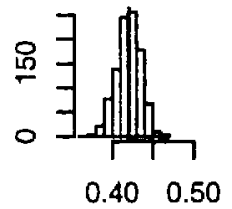

10
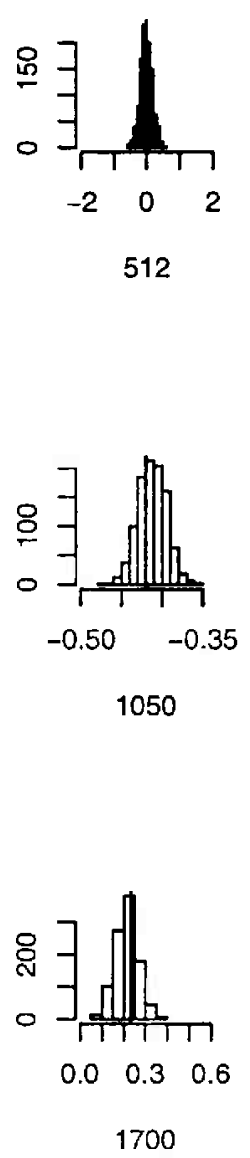
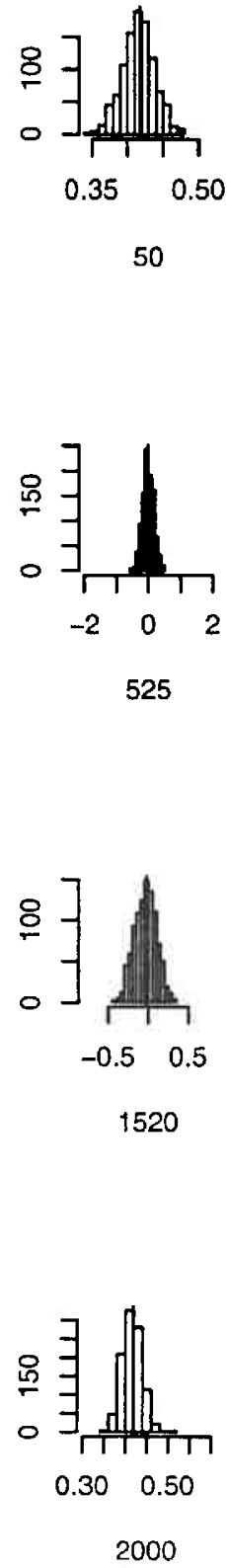
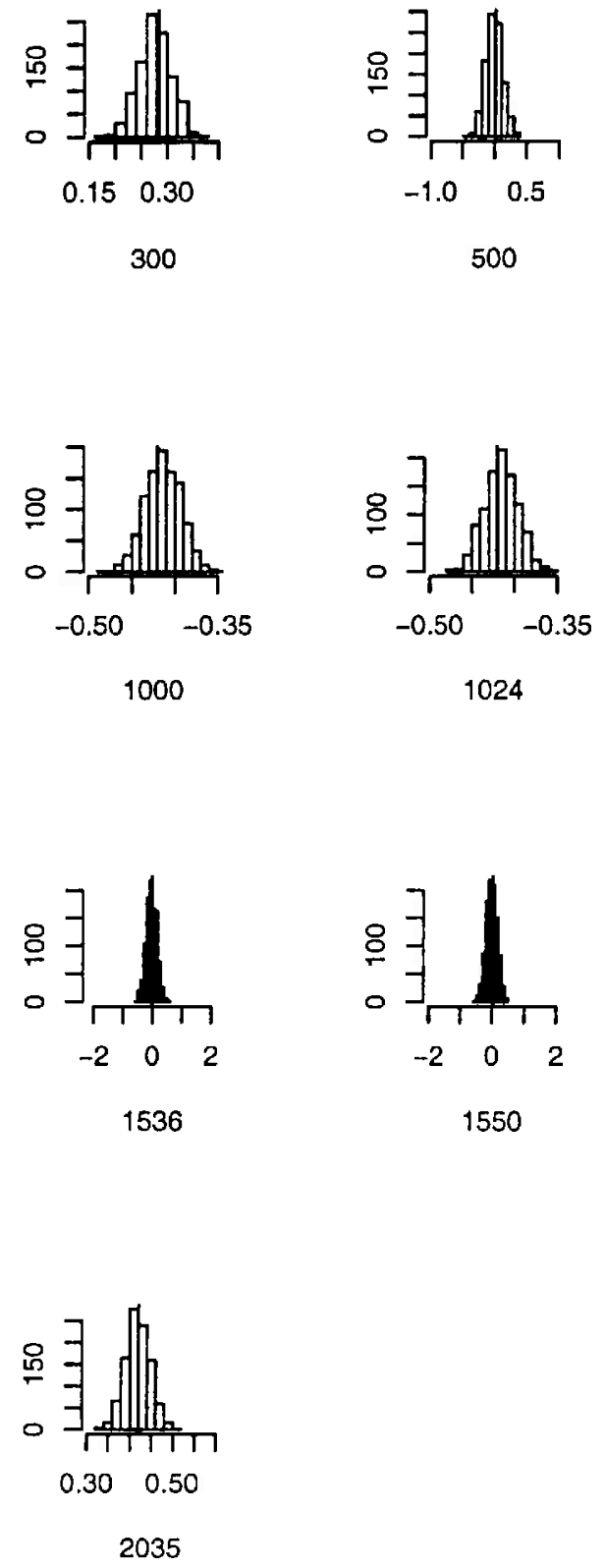

Figura B.17: Histogramas de $\hat{\delta}_{2}(t)$, Caso 2, nos diferentes valores de $t$. 

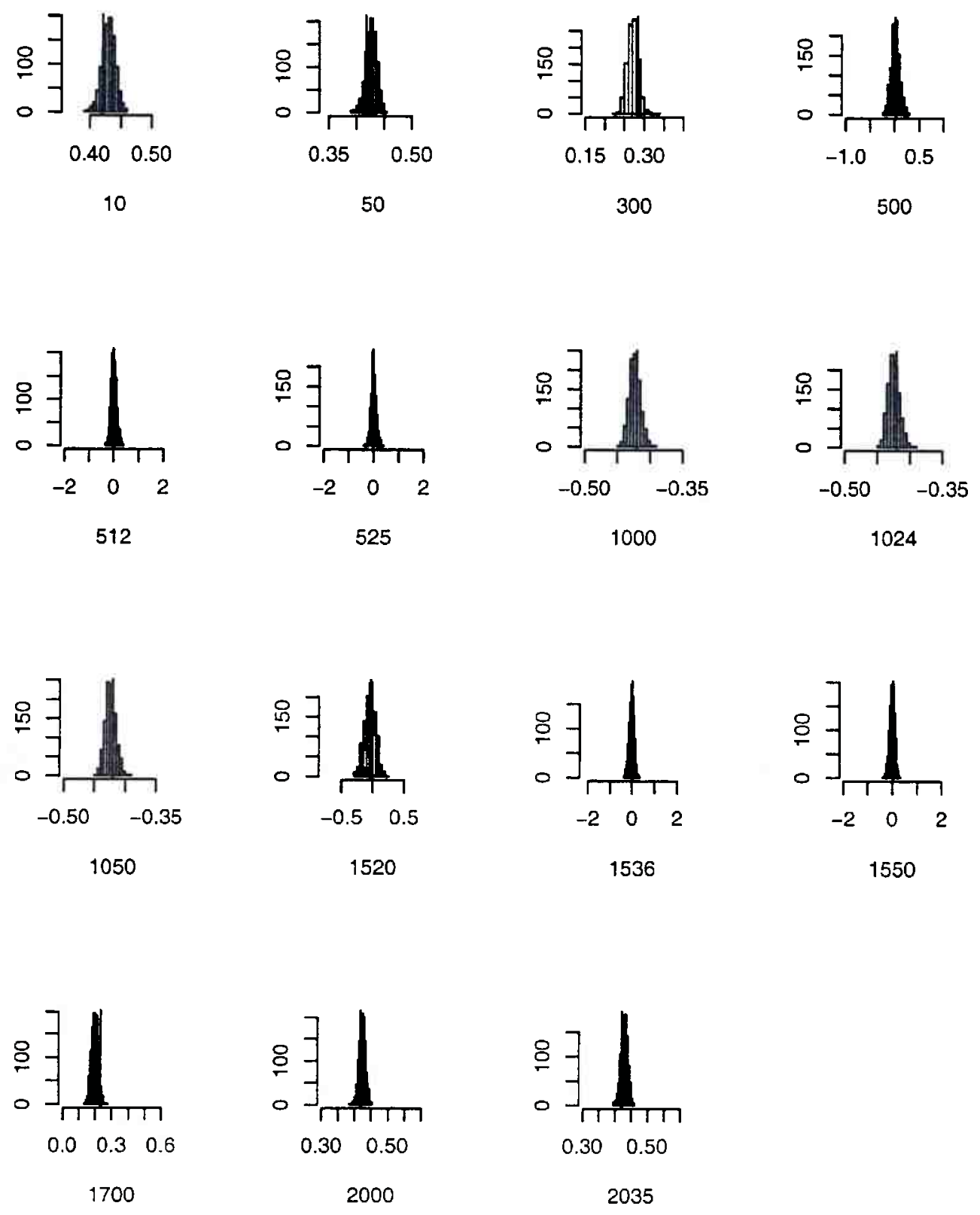

Figura B.18: Histogramas de $\tilde{\delta}_{2}^{(L)}(t)$, Caso 2, nos diferentes valores de $t$. 

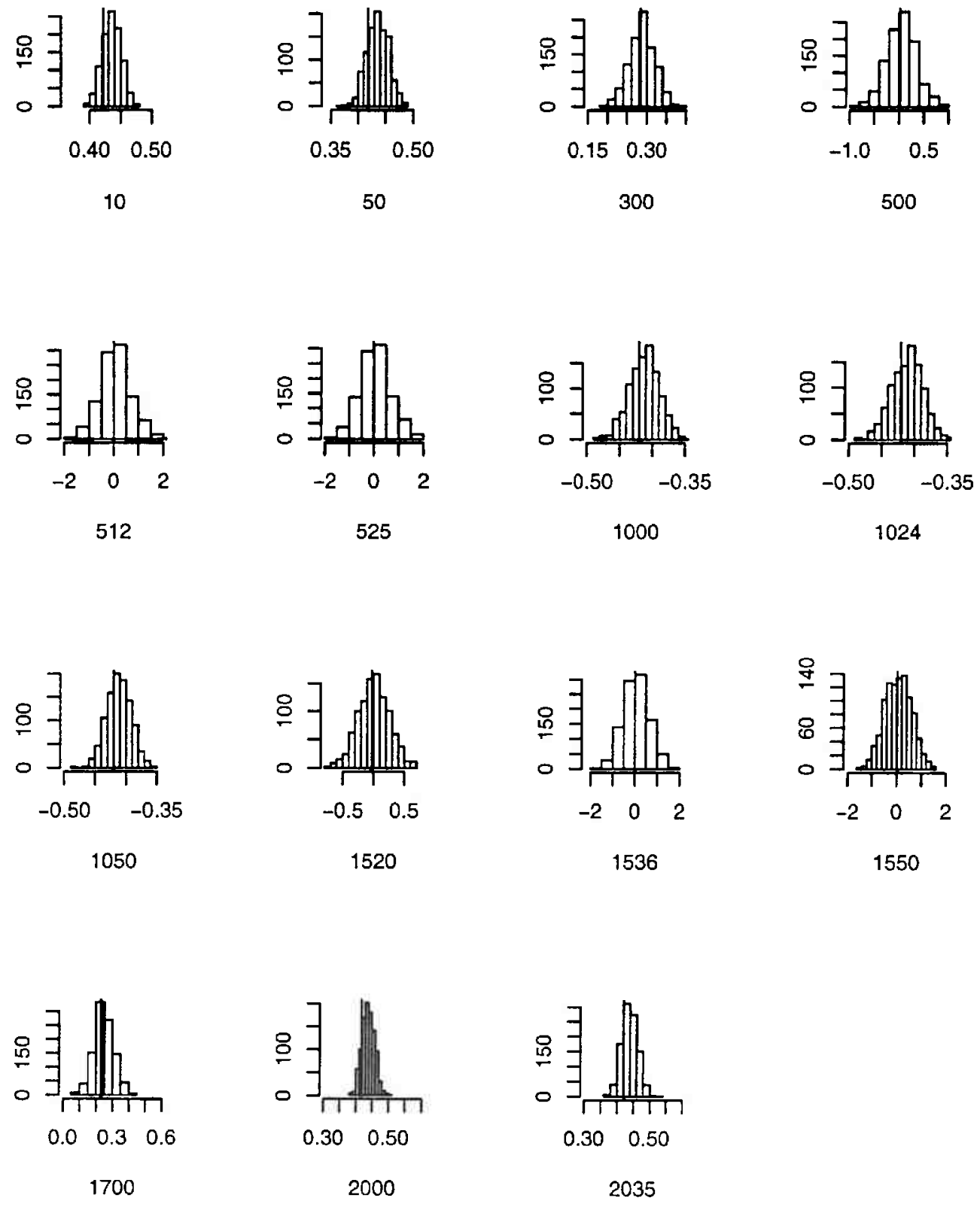

Figura B.19: Histogramas de $\tilde{\delta}_{2}(t)$, Caso 2, nos diferentes valores de $t$. 

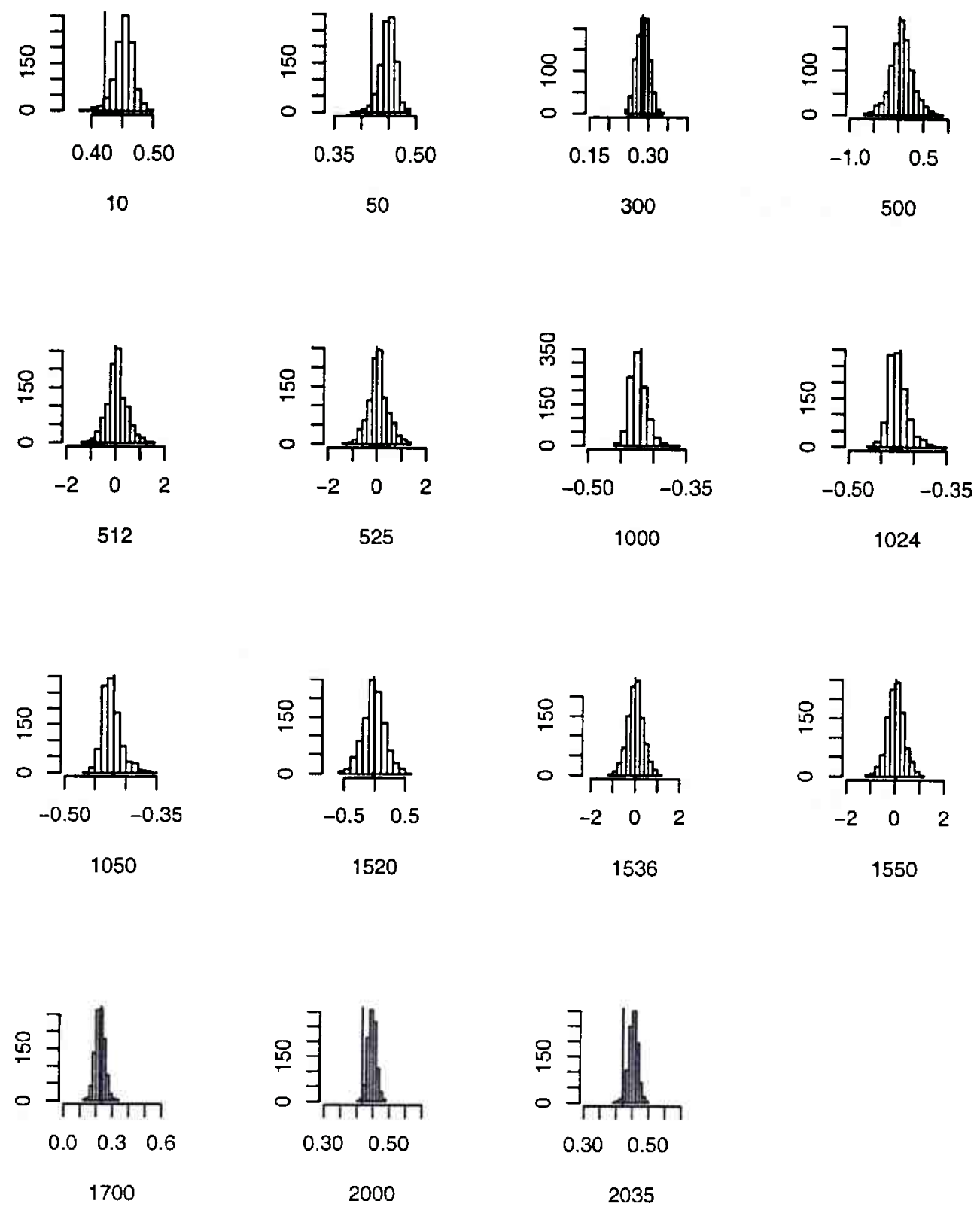

Figura B.20: Histogramas de $\tilde{\delta}_{2}^{(L)}(t)$, Caso 2, nos diferentes valores de $t$. 


\section{Apêndice $\mathrm{C}$}

\section{Resultados}

Nesta seção apresentamos os resultados do ajuste do modelo de função de transferência para as séries de Chuva (em $\mathrm{mm}$ ) e Temperatura Máxima $\left(e m^{\circ} \mathrm{C}\right)$ apresentados na Seção 4.4

Na Figura C.1 estão os resultados dos Modelos $M_{1}$. As Figuras (a) e (b) são as estimativas $\hat{\delta}_{1}(t)$ e $\hat{\omega}_{0}(t),(\mathrm{c})$ e (d) as estimativas $\hat{\delta}_{1}^{(L)}(t)$ e $\hat{\omega}_{0}^{(L)}(t)$ (linha cheia) e intervalo bootstrap de $95 \%$ (linha pontilhada), (e) e (f) as estimativas $\tilde{\delta}_{1}(t)$ e $\tilde{\omega}_{0}(t)$ e $(\mathrm{g})$ e $(\mathrm{h})$ as estimativas $\tilde{\delta}_{1}^{(L)}(t)$ e $\tilde{\omega}_{0}^{(L)}(t)$ (linha cheia) e intervalo bootstrap de $95 \%$ (linha pontilhada).

Na Figura C.2 estão os resultados dos Modelos $M_{2}$. As Figuras (a), (b) e (c) são as estimativas $\hat{\delta}_{1}(t), \hat{\omega}_{0}(t)$ e $\hat{\omega}_{1}(t),(\mathrm{d}),(\mathrm{e})$ e (f) as estimativas $\hat{\delta}_{1}^{(L)}(t), \hat{\omega}_{0}^{(L)}(t)$ e $\hat{\omega}_{1}^{(L)}(t)$ (linha cheia) e intervalo bootstrap de $95 \%$ (linha pontilhada), (g), (h) e (i) as estimativas de $\tilde{\delta}_{1}(t), \tilde{\omega}_{0}(t)$ e $\tilde{\omega}_{1}(t),(\mathrm{j}),(\mathrm{k})$ e $(\mathrm{l})$ as estimativas $\hat{\delta}_{1}^{(L)}(t), \hat{\omega}_{0}^{(L)}(t)$ e $\hat{\omega}_{1}^{(L)}(t)$ (linha cheia) e intervalo bootstrap de $95 \%$ (linha pontilhada).

Na Figura C.3 estão os resultados dos Modelos $M_{3}$. As Figuras (a), (b) e (c) 
são as estimativas de $\hat{\delta}_{1}(t), \hat{\delta}_{2}(t)$ e $\hat{\omega}_{0}(t),(\mathrm{e}),(\mathrm{f})$ e (g) as estimativas $\hat{\delta}_{1}^{(L)}(t), \hat{\delta}_{2}^{(L)}(t)$ e $\hat{\omega}_{0}^{(L)}(t)$ (linha cheia) e intervalo bootstrap de $95 \%$ (linha pontilhada), (h), (i) e (j) as estimativas $\tilde{\delta}_{1}(t), \tilde{\delta}_{2}(t)$ e $\tilde{\omega}_{0}(t)$ e, (k), (l) e (m) as estimativas $\hat{\delta}_{1}^{(L)}(t), \hat{\delta}_{2}^{(L)}(t)$ e $\hat{\omega}_{0}{ }^{(L)}(t)$ (linha cheia) e intervalo bootstrap de $95 \%$ (linha pontilhada).

Nos resultados dos modelos ajustados ajustados notamos que, após a limiarização as curvas são, como esperado, mais suaves, incluindo neste comentário os intervalos bootstrap. Comparando as estimativas do primeiro com a do segundo estágio vemos, em $\omega_{0}(t)$, uma variação na função por volta de $t=500$. 


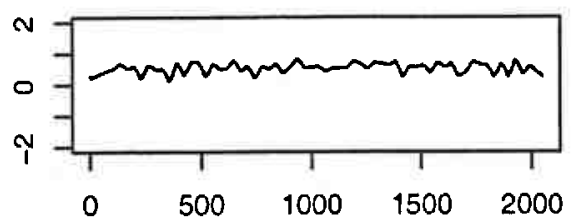

(a)

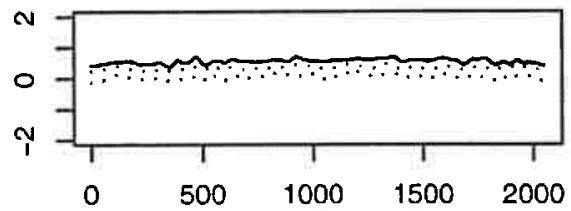

(c)

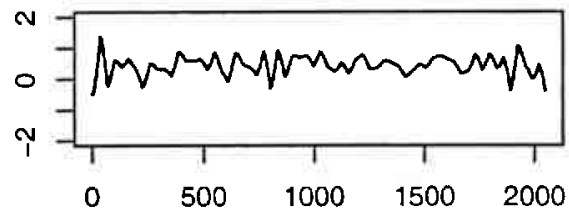

(e)

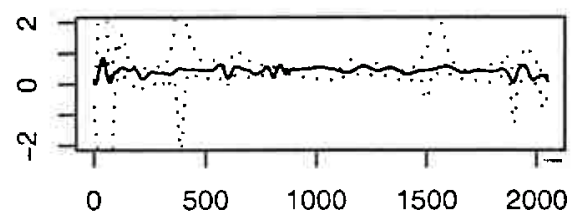

(g)

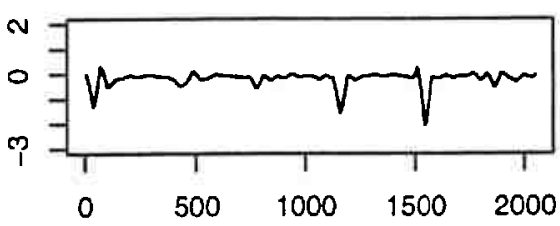

(b)

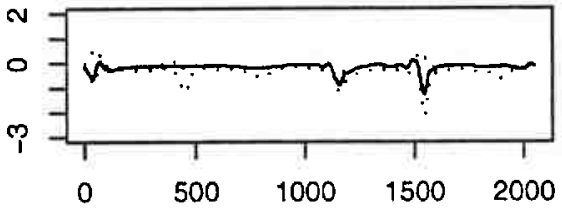

(d)

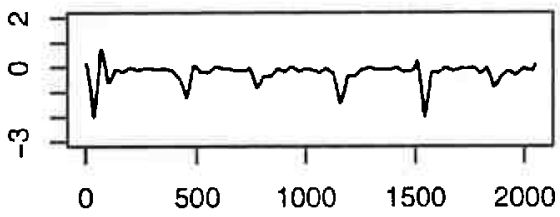

(f)

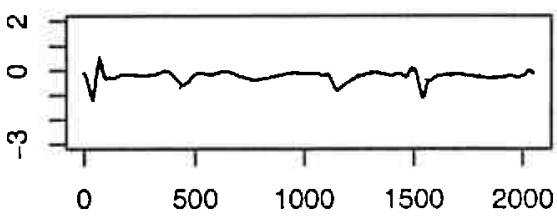

(h)

Figura C.1: Resultados do ajuste do modelo 1. 


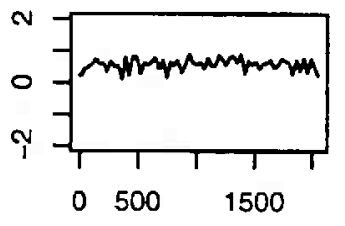

(a)

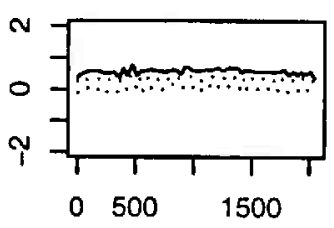

(d)

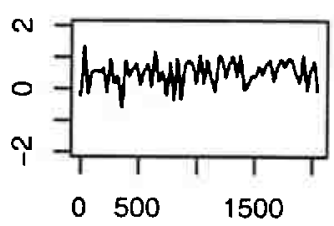

(g)

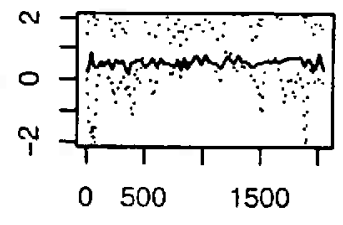

(j)

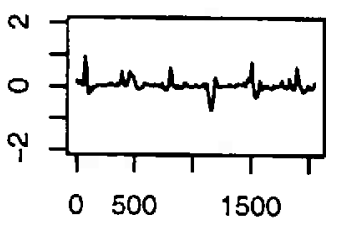

(b)

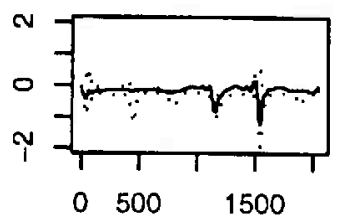

(e)

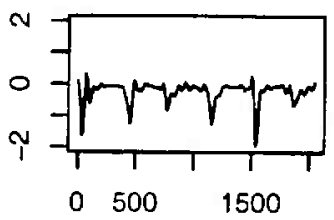

(h)

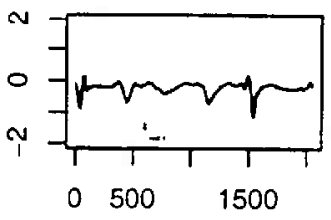

(k)

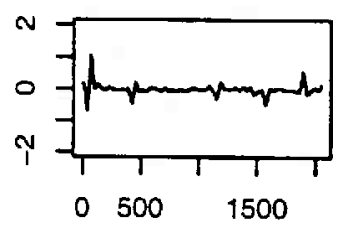

(c)

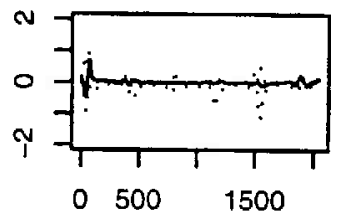

(f)

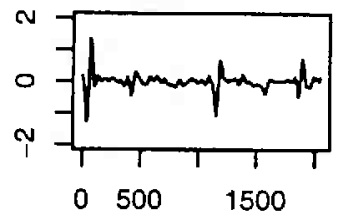

(i)

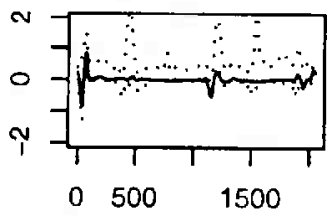

(I)

Figura C.2: Resultados do ajuste do modelo 2. 


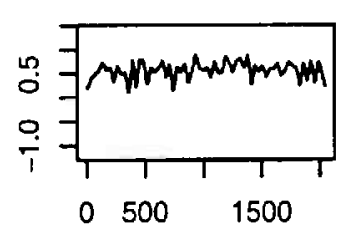

(a)

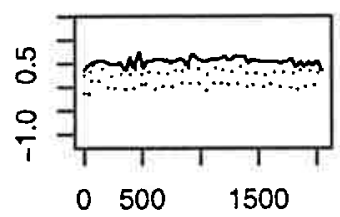

(d)

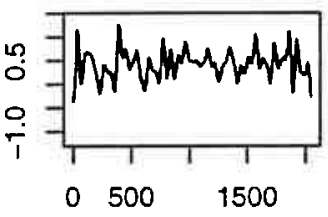

(g)

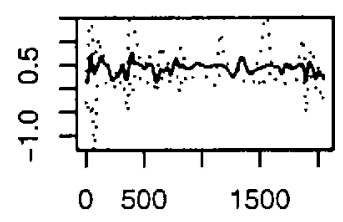

(j)

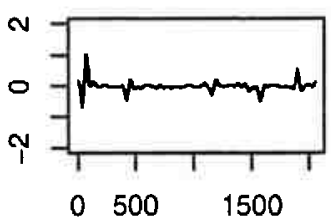

(b)

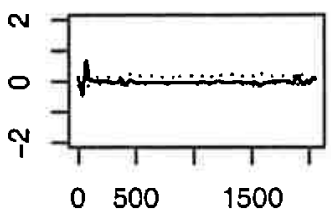

(e)

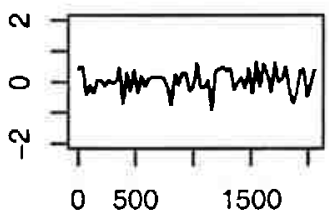

(h)

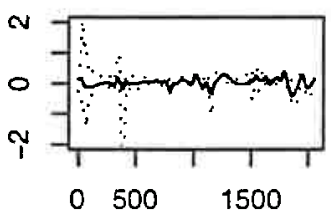

(k)

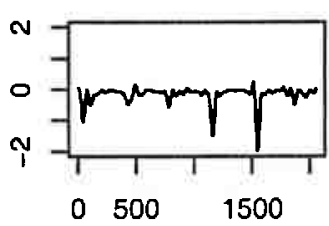

(c)

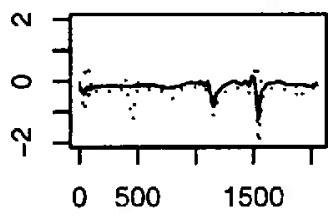

(f)

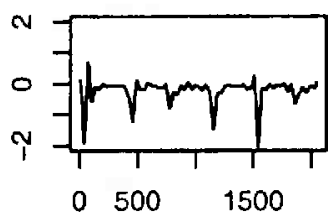

(i)

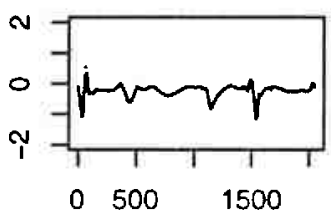

(I)

Figura C.3: Resultados do ajuste modelo 3. 


\section{Apêndice D}

\section{Programa}

O programa abaixo foi usado para o ajuste do modelo 3 do Capítulo 5 .

Seja $\mathrm{x}$ a série de entrada e y a série de saída. N é o tamanho da série. Psi é a matriz que faz a decomposição em ondaletas até o nível $j^{*}$ e, $p=2^{j^{*}}$. B1 é o número de amostras Bootstrap e pb o valor de $\alpha$ para o cálculo do Intervalo Bootstrap.

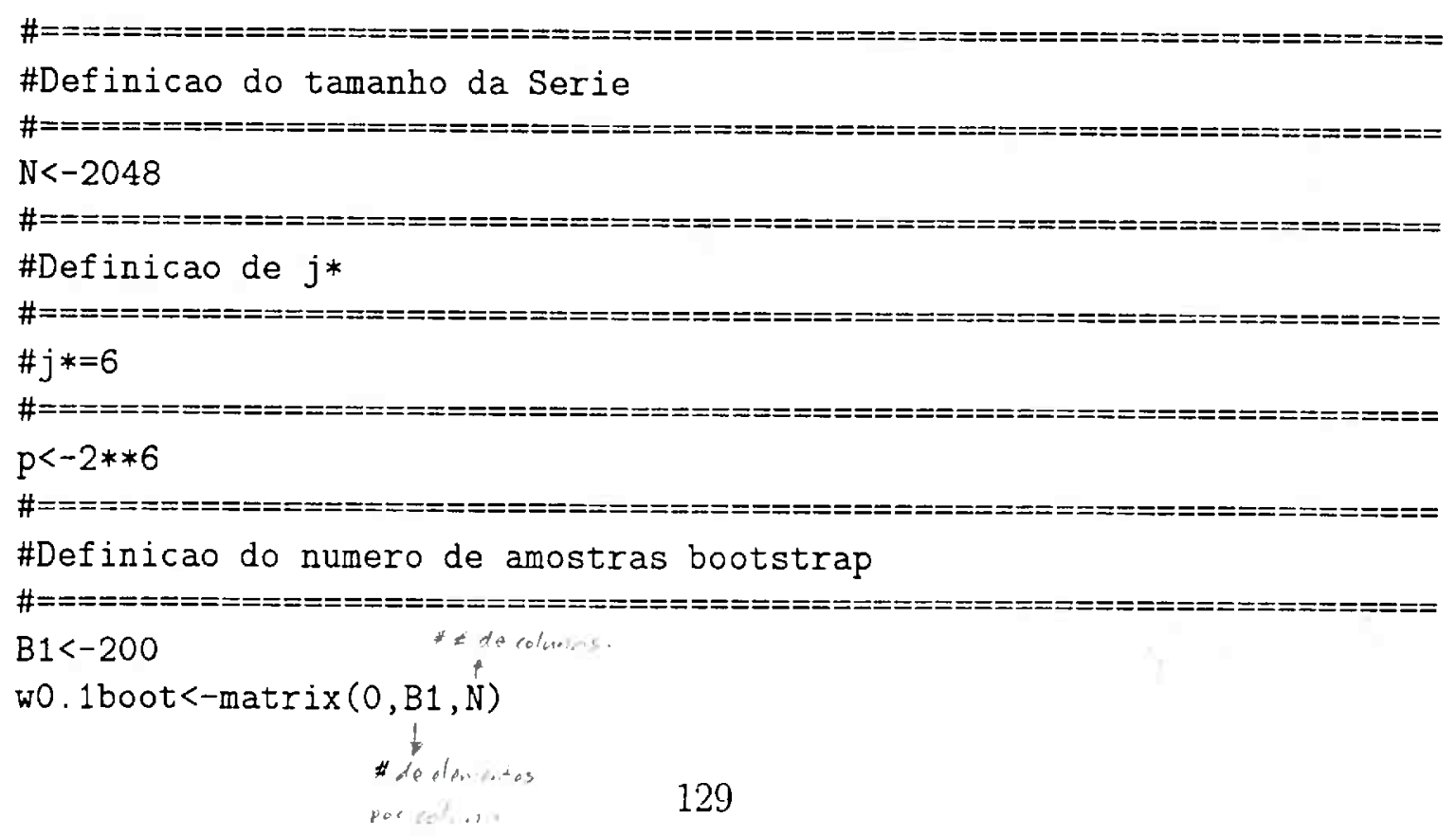




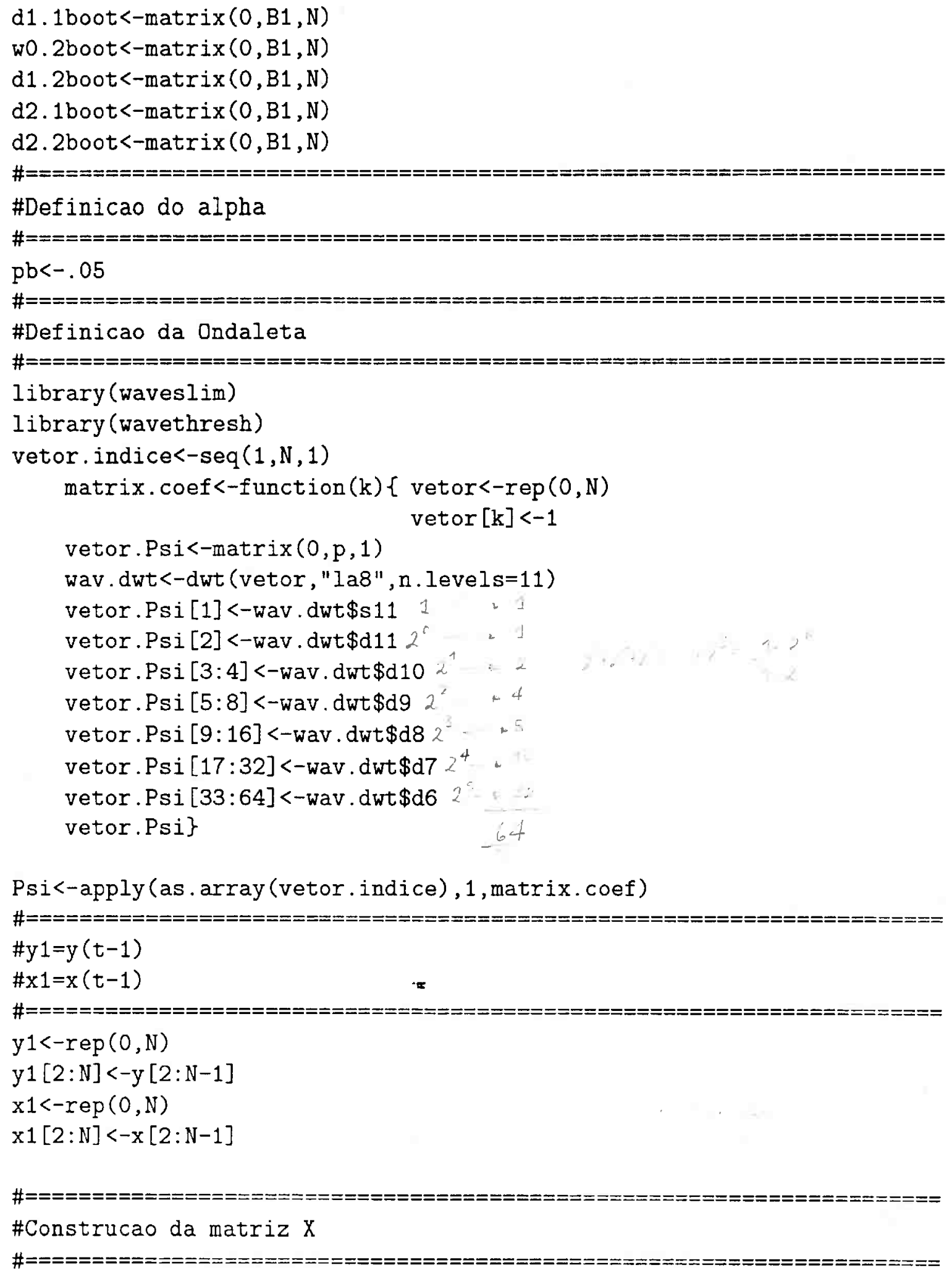




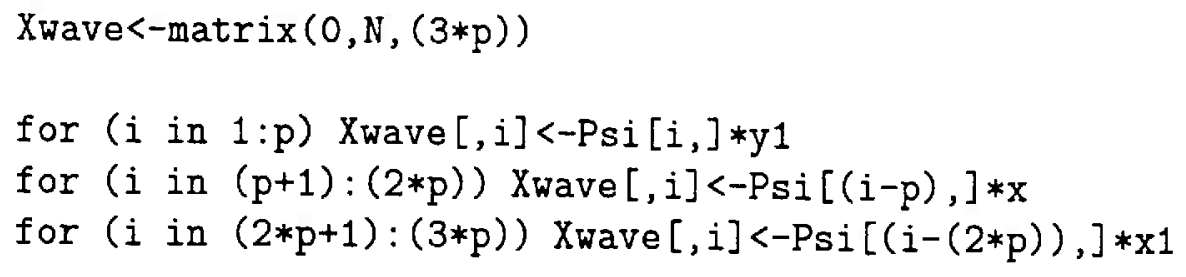

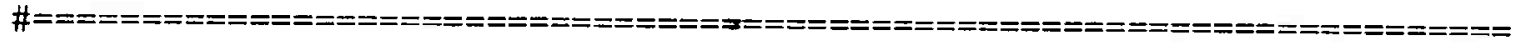
\#Calculo do Threshold

\#MAD

\#Sure

\#threshold suave

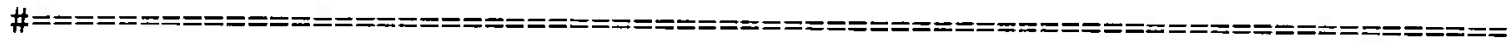

d1.1wd<-wd(d1.1,filter number=8, family="DaubLeAsymm" , bc="periodic") d1.1th<-threshold. wd (d1.1wd, levels=1:10, type="soft",policy="universal") d1. $1 \mathrm{TH}<-$ wr $(\mathrm{d} 1.1 \mathrm{th})$ 
w0. $1 \mathrm{wd}<-$ wd (w0.1, filter. number=8, family="DaubLeAsymm", bc="periodic")

w0.1 th<-threshold. wd (w0.1wd, levels=1:10, type="soft", policy="universal") w0.1 TH<-wr (w0.1th)

d2. $1 \mathrm{wd}<-w d$ (d2 1 , filter. number $=8$, family="DaubLeAsymm" , bc="periodic")

d2.1th<-threshold. wd (d2.1 wd, levels=1:10, type="soft", policy="universal")

d2. $1 \mathrm{TH}<-\mathrm{wr}(\mathrm{d} 2.1 \mathrm{th})$

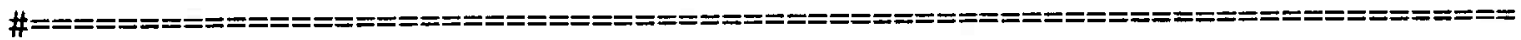

\# Calculo do vetor de erros do primeiro estagio

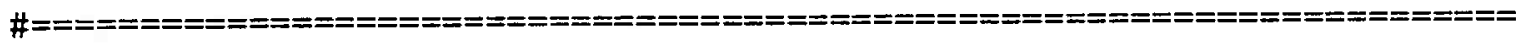

yh.thres $1<-\operatorname{matrix}(0, \mathrm{~N}, 1)$

yh. thres $1[1]<-$ w0 .1 TH $[1] * x[1]$

yh. thres $1[2]<-d 1.1 \mathrm{TH}[2] * \mathrm{y}[1]+$ w0 $0.1 \mathrm{TH}[2] * \mathrm{x}[2]$

for ( $i$ in $3: N$ )

yh . thres $1[i]<-d 1.1 \mathrm{TH}[\mathrm{i}] * \mathrm{y}[\mathrm{i}-1]+$ w0.1TH $[\mathrm{i}] * \mathrm{x}[\mathrm{i}]+\mathrm{d} 2.1 \mathrm{TH}[\mathrm{i}] * \mathrm{y}[\mathrm{i}-2]$

errothres $1<-r e p(0, N)$

\#Calculo do Erro*

errothres $1<-y$-yh.thres 1

errothres1<-errothres1-mean(errothres1)

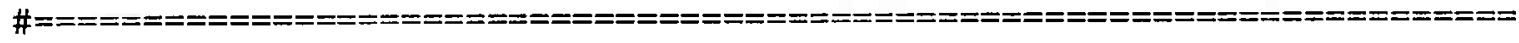

\# Bootstrap para o 10. Estagio

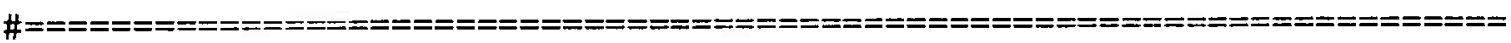

\#Bootstrap

d1.1star<-rep $(0, p)$

w0.1star<-rep $(0, p)$

d2. 1 star $<-r e p(0, p)$

d1. 1 star $1<-\operatorname{rep}(0, p)$

w0.1star $1<-\operatorname{rep}(0, p)$

d2. 1star1<-rep $(0, p)$

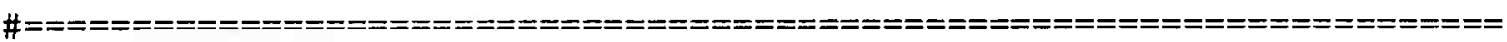

\#Inicio do ciclo de repeticao bootstrap 


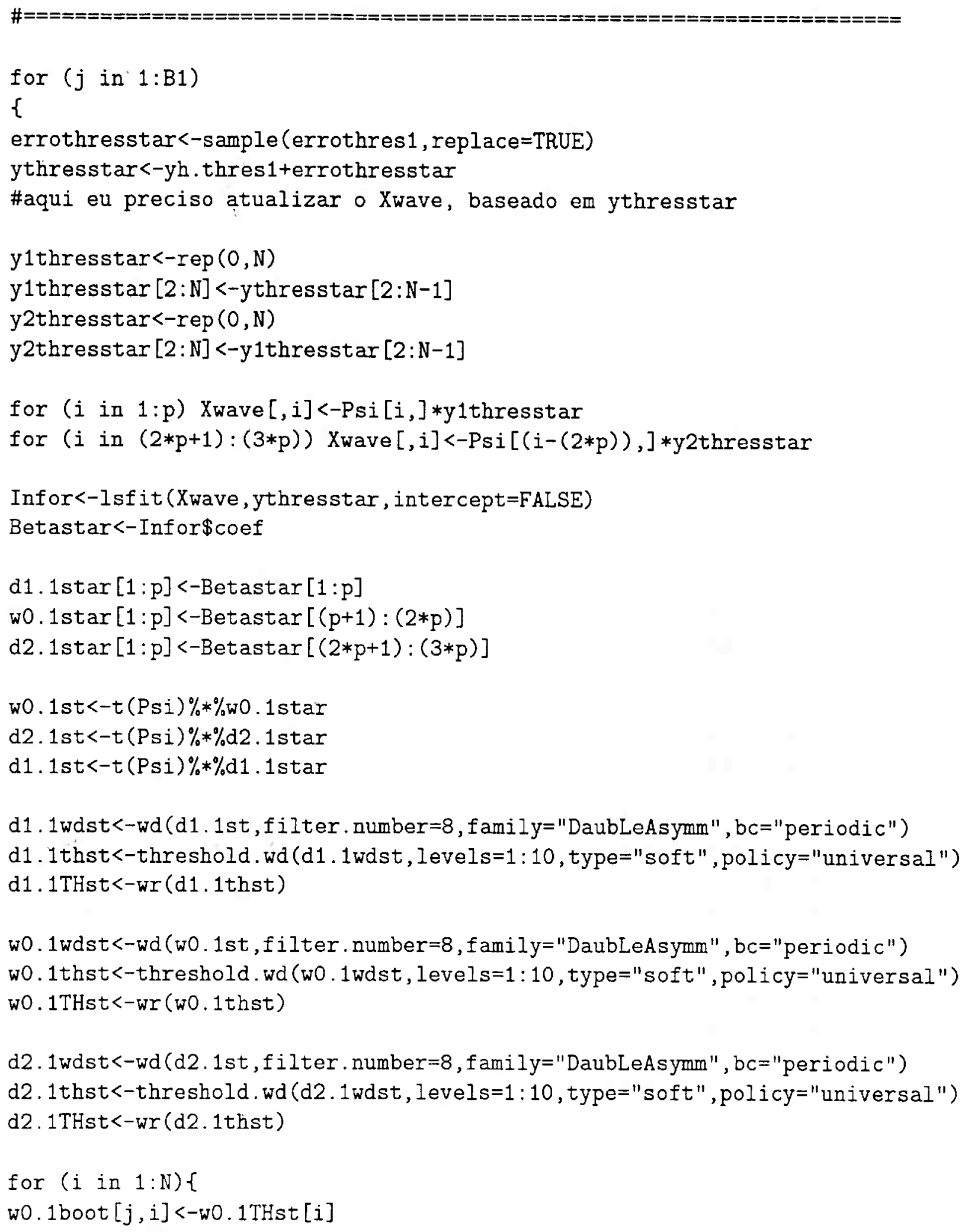




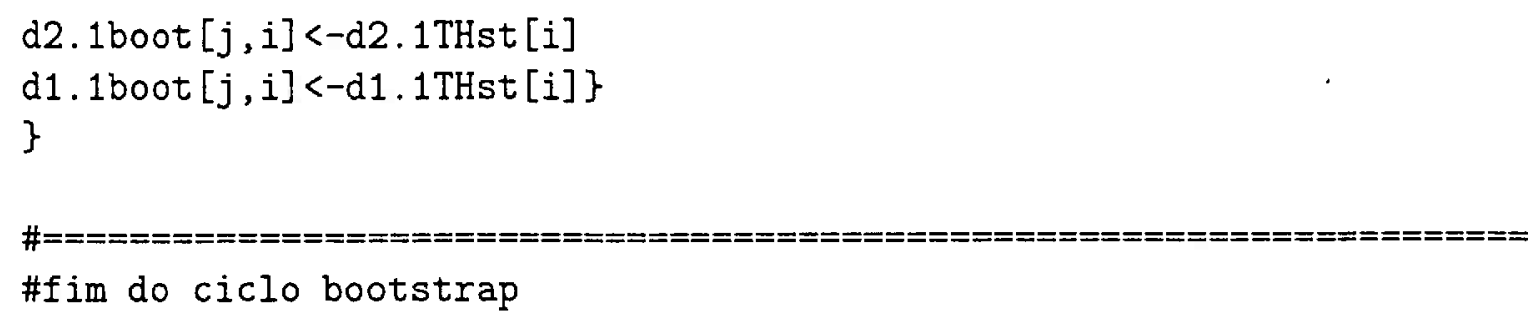


for ( $i$ in $3: N$ )

$\mathrm{yh}[\mathrm{i}]<-\mathrm{d} 1.1 \mathrm{TH}[\mathrm{i}] * \mathrm{y}[\mathrm{i}-1]+\mathrm{w} 0.1 \mathrm{TH}[\mathrm{i}] * \mathrm{x}[\mathrm{i}]+\mathrm{d} 2.1 \mathrm{TH}[\mathrm{i}] * \mathrm{y}[(\mathrm{i}-2)]$

yh1<-rep $(0, N)$

yh1 $[2: \mathrm{N}]<-\mathrm{yh}[2: \mathrm{N}-1]$

$\operatorname{yh} 2<-\mathrm{rep}(0, \mathrm{~N})$

yh2 $[2: \mathrm{N}]<-\mathrm{yh} 1[2: \mathrm{N}-1]$

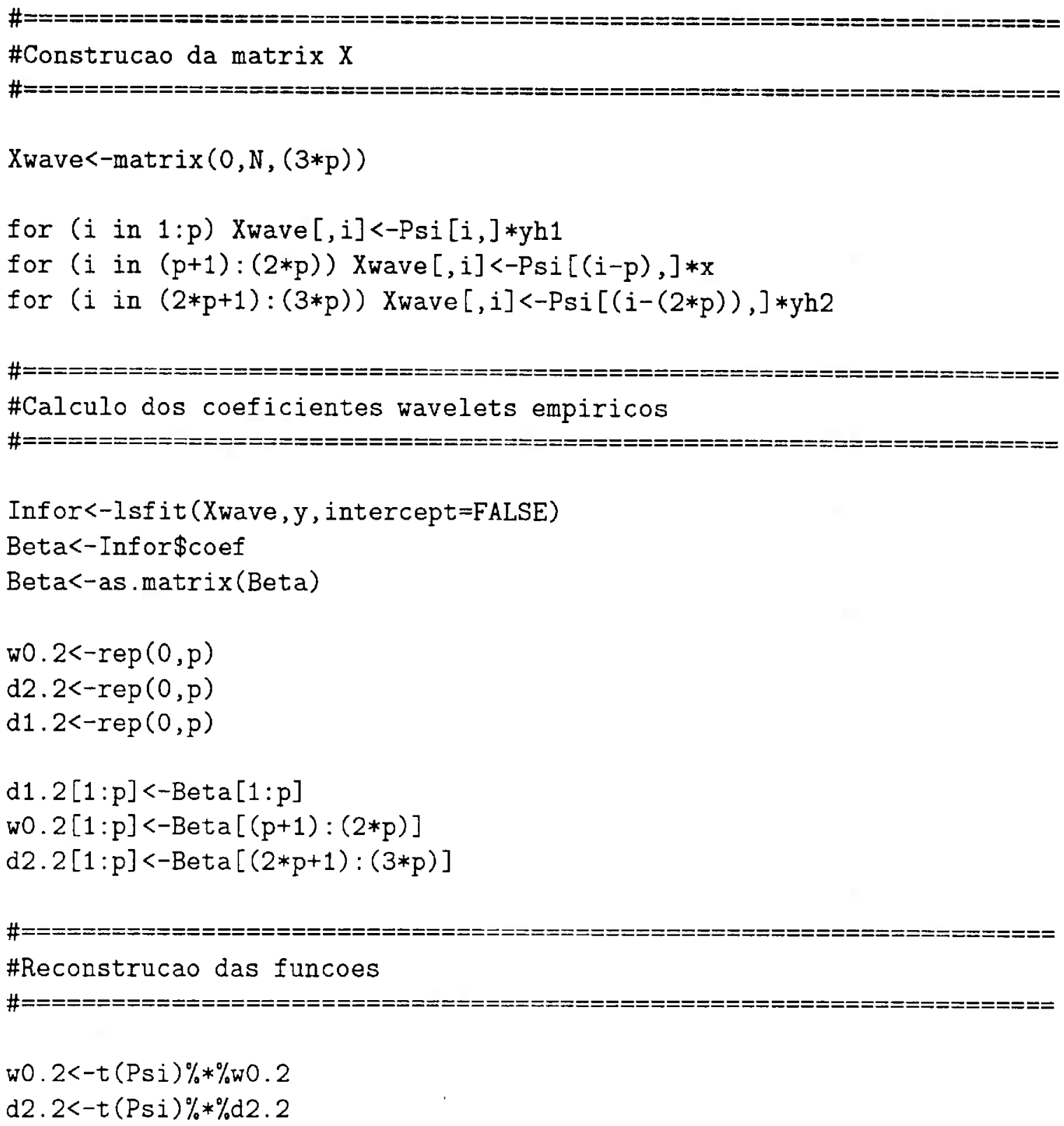




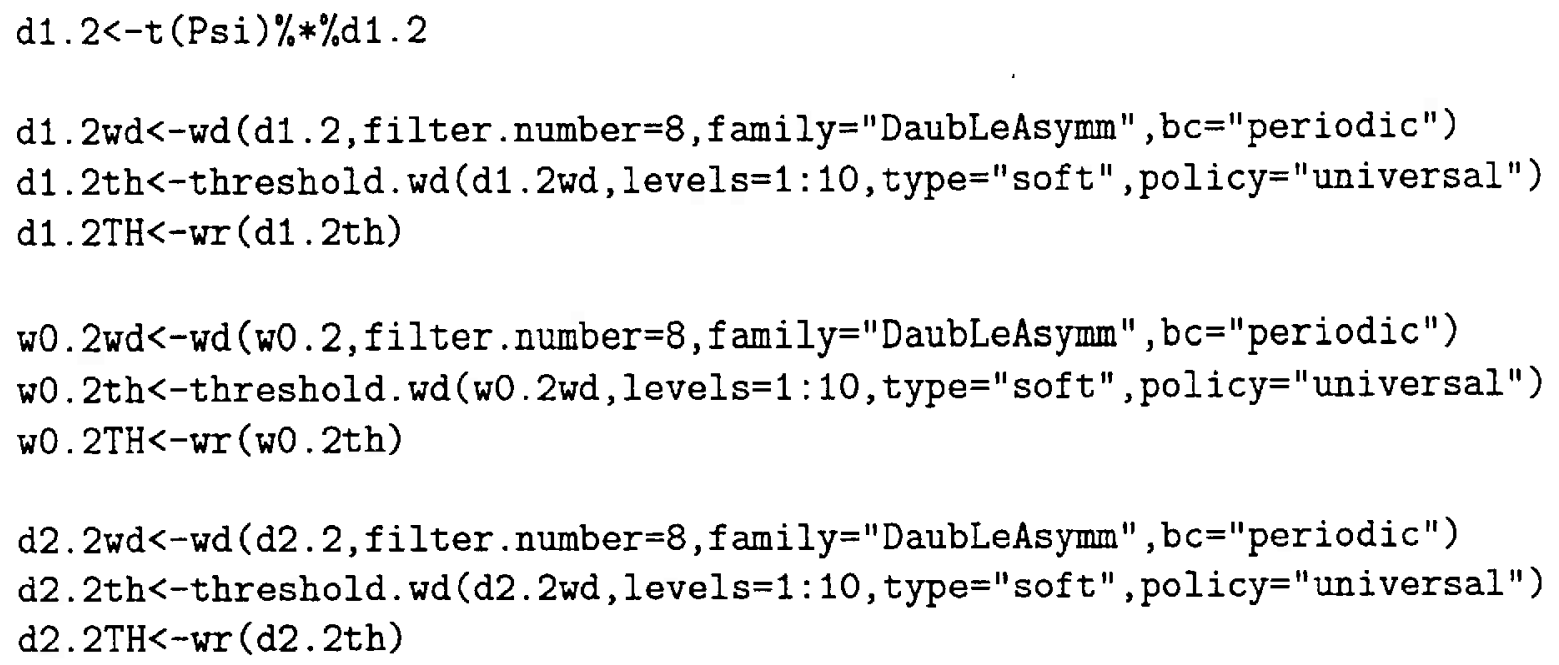




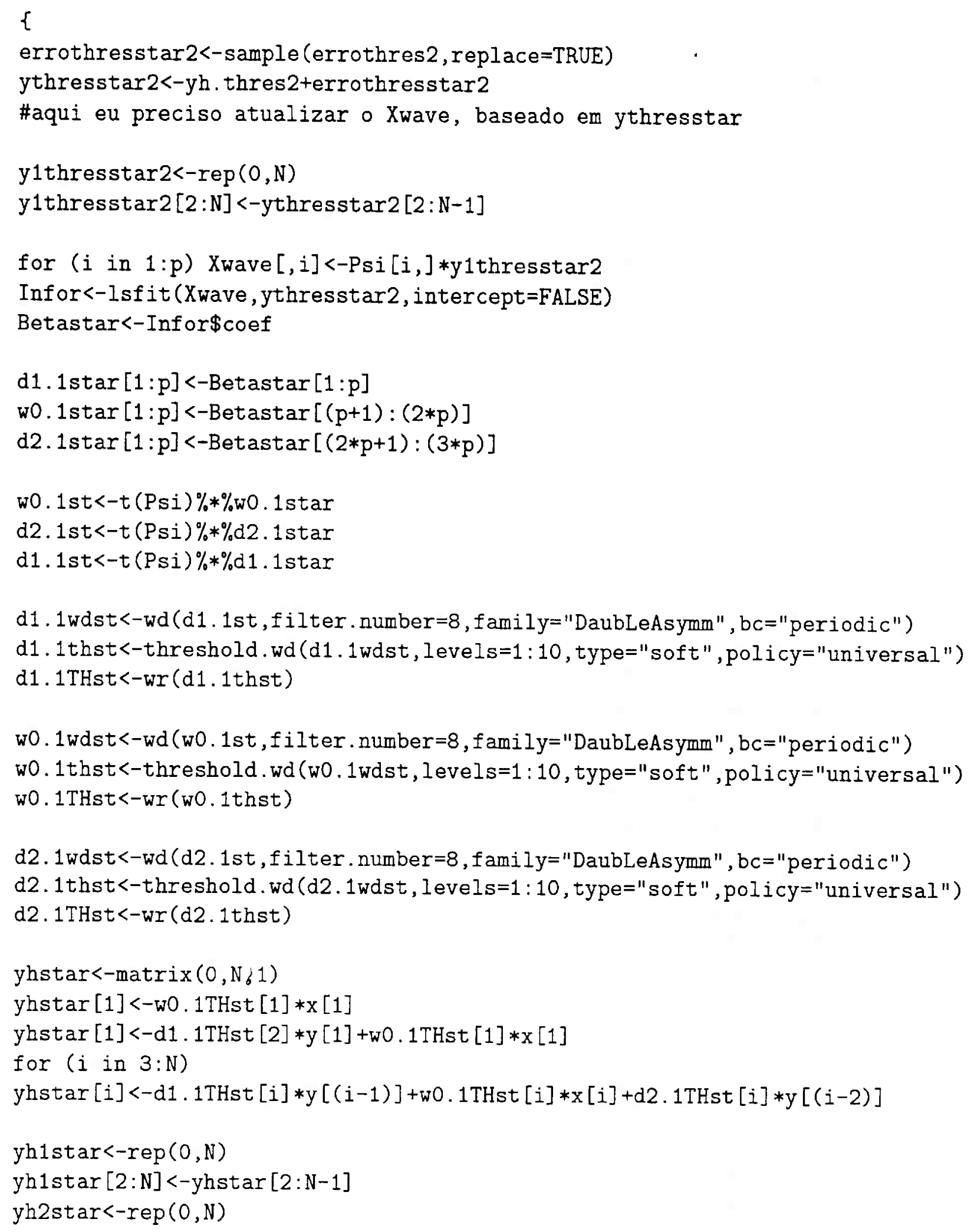


yh2star $[2: \mathrm{N}]<-\operatorname{yh} 1 \mathrm{star}[2: \mathrm{N}-1]$

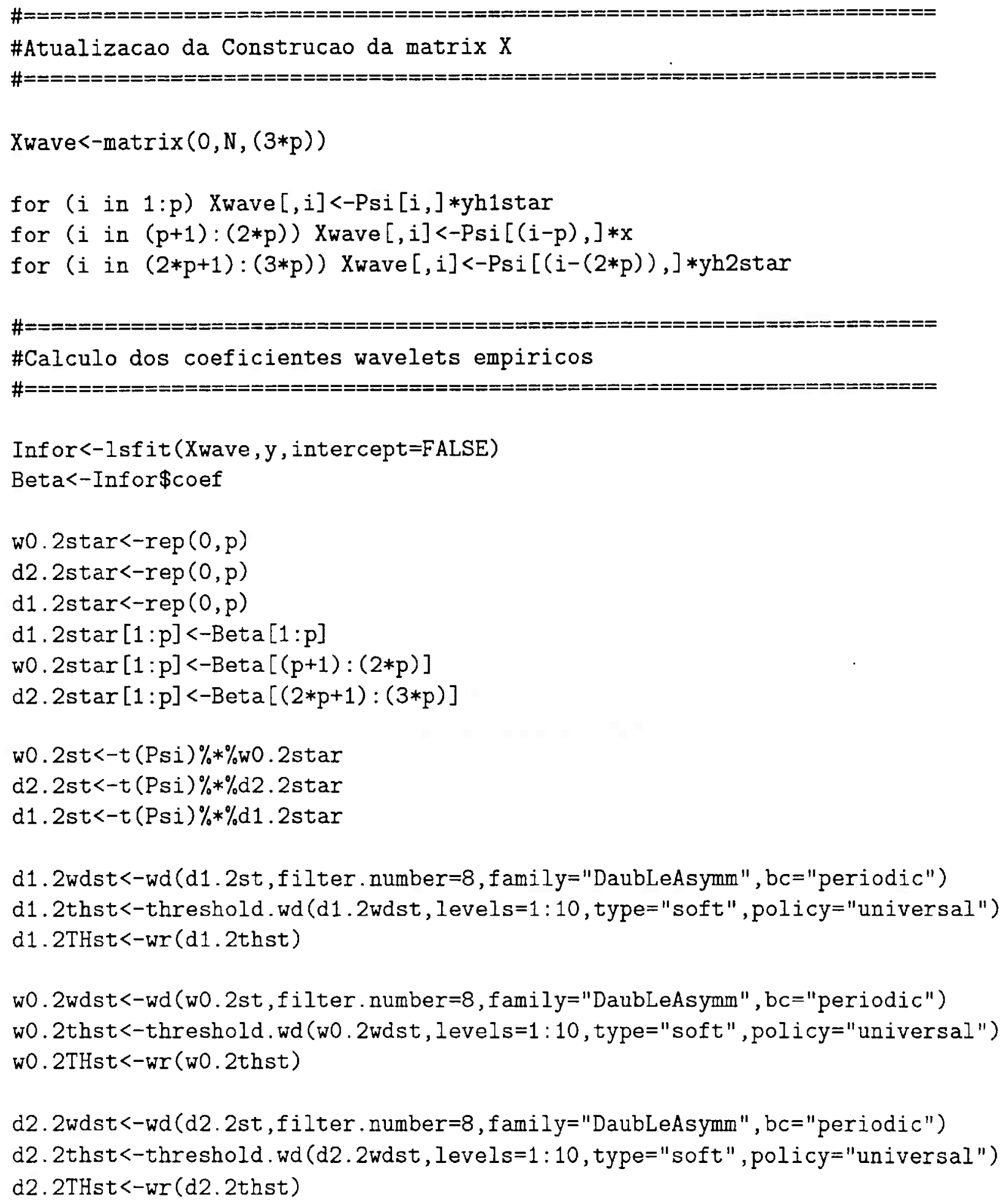




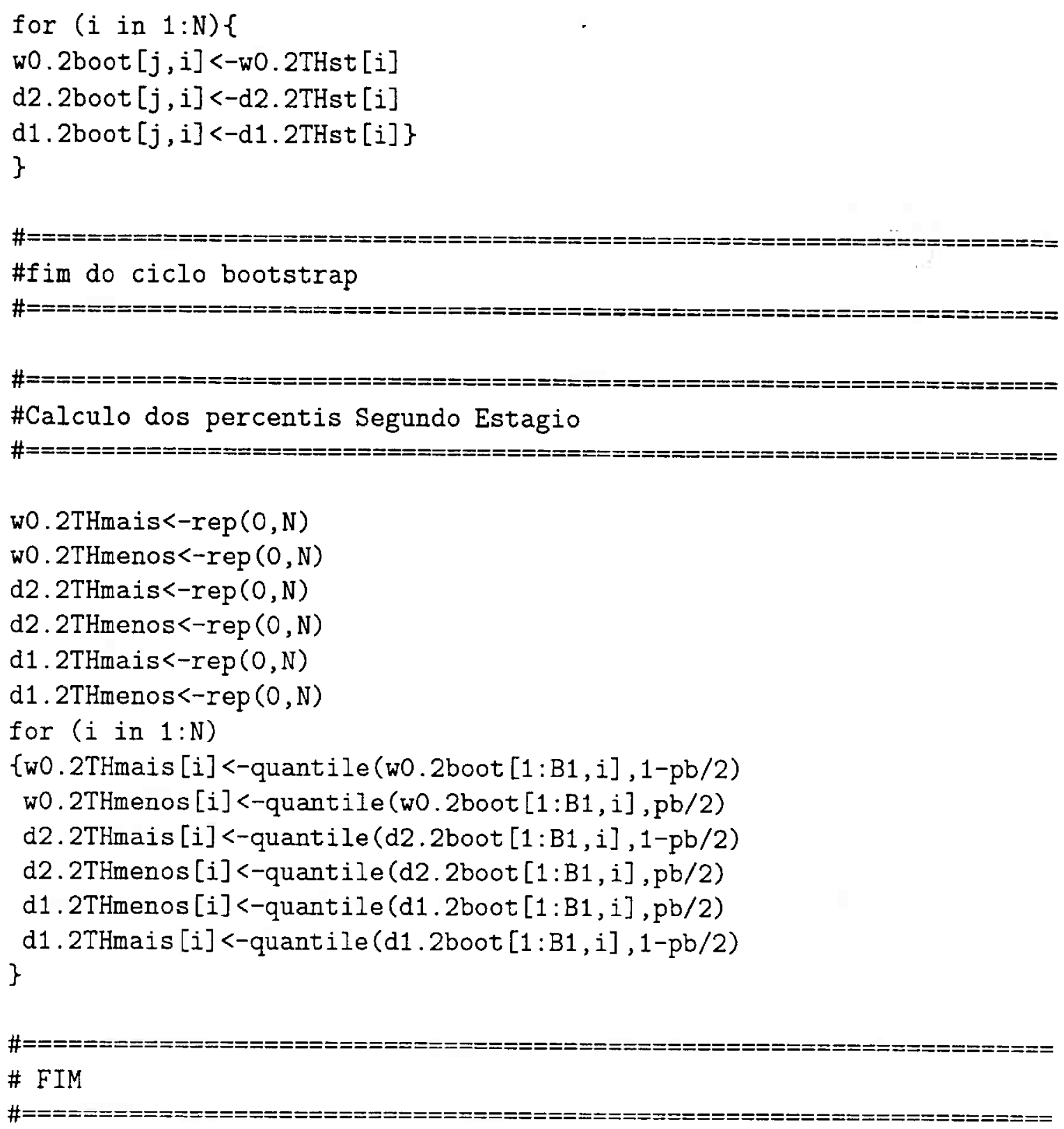




\section{Referências}

Bentkus, R. e Rudzkis, R. (1980). On exponencial estimates of the distribution of random variables. Liet. Mat. Rink, 20, 15-30.

Box, G. E. P., Jenkins G. M. and Reinsel, G. C. (1994). Time Series Analysis: Forecasting and Control. Englewood Cliffs: Prentice-Hall.

Chiann, C. and Morettin, P.A. (1999). Estimation of Time Varying Linear Systems. Statistical Inference for Stochastic Processes, 2, 253-285.

Chiann, C. and Morettin, P.A. (2005). Time Domain Estimation of Time Varying Linear Systems. A aparecer, J. Nonparametrics Statistics .

Chui, C.K. (1992). An Introduction to Wavelets. Texas: Academic Press.

Dahlhaus, R. (1996). On the Kullback-Leibler Information Divergence of Locally Stationary Processes. Stochastic Processes and their Applications, 62, 139168.

Dahlhaus, R. (1996). Asymptotic Statistical Inference for Nonstationary Processes with Evolutionary Spectra. Athens Conference on Applied Probability and Time Series. Lecture Notes in Statistics, 115, 145-159.

Dahlhaus, R. (1997). Fitting Time Series Models to Nonstationary Processes. The Annals of Statistics, 25, 1-37. 
Dahlhaus, R. and Giraitis, L. (1998). On the Optimal Segment Length for Parameter Estimates for Locally Stationary Time Series. Journal of Time Series Analysis, 19, 629-655.

Dahlhaus, R., Neumann, M.H. and von Sachs, R. (1999). Nonlinear wavelet estimation of time-varying autoregressive process. Bernoulli, 5, 873-906.

Dahlhaus, R. (2000). A Likelihood Approximation for Locally Stationary Processes. The Annals of Statistics, 28, 1762-1794.

Daubechies, I. (1988). Orthonormal Bases of Compactly Supported Wavelets. Communications on Pure and Applied Mathematics, 41, 909-996.

Donoho, D., Johnstone, I., Kerkyacharian, G. and Picard, D. (1995). Wavelet shrinkage: asymptopia? (with discussion) Journal of the Royal Statistical Society, Series B, 57, 301-337.

Efron, B. and Tibshirani, R. (1993) An Introduction to the Bootstrap. New York: Chapman Hall.

Fuller, W. A. (1996). Introduction to Statistical Time Series. New York: Wiley. Johnson, N. and Kotz, S. (1970). Distibutions in Statistics, Continuos Univariate Distribuitions. New York: Wiley.

Künsch, H. (1995). A Note on Causal Solutions for Locally Stationary ARprocess. Manuscrito, ETH Zürich.

Mallat, S., Papanicolau, G. and Zanhg Z. (1998). Adaptive Covariance Estimation of Locally Stationary Process. The Annals of Statistics, 26, 1-47.

Meyer, Y. (1993). Wavelets: Algoritms and Applications. Philadelphia: SIAM. 
Morettin, P.A. (1999). Ondas e Ondaletas da Análise de Fourier à Análise de Ondaletas. São Paulo: EDUSP.

Neumann, M. (1996). Spectral Density Estimation via Nonlinear Wavelet Methods for Stationary Non-Gaussian Time Series. Journal of Time Series Analysis, 17, 601-633.

Noble, B. and Daniel, J.W. (1988). Applied Linear Algebra. Englewood Cliffs: Prentice-Hall.

Percival, D. B. and Walden, A. T. (2000). Wavelets Methods for Time Series. London: Cambridge University Press.

Priestley, M. (1981). Spectral Analysis and Time Series. London: Academic Press.

Rudzkis, R., Saulis, L. and Statulevicius, V. (1978). A general lemma on probabilities for large deviations. Lithuanian Mathematical Journal, 18, 226-238.

Triebel, H. (1992). Theory of Function Spaces II. Basel: Birkhaüser Verlag.

Vidakovic, B. (1999). Statistical Modelling by Wavelets. Durham: Wiley. 